PREDICTING THE HALL-PETCH EFFECT IN FCC METALS

USING NON-LOCAL CRYSTAL PLASTICITY

\begin{abstract}
A Dissertation
Presented to

The Academic Faculty

by

William A. Counts

In Partial Fulfillment

of the Requirements for the Degree

Doctor of Philosophy in Materials Science and Engineering in the School of Materials Science and Engineering
\end{abstract}

Georgia Institute of Technology

May 2007 


\section{PREDICTING THE HALL-PETCH EFFECT IN FCC METALS USING NON-LOCAL CRYSTAL PLASTICITY}

Approved by:

Dr. Ashok Saxena, Advisor

School of Materials Science and Engineering

Georgia Institute of Technology

Dr. Mo Li

School of Materials Science and Engineering

Georgia Institute of Technology

Dr. Tom Sanders

School of Materials Science and Engineering Georgia Institute of Technology
Dr. Corbett Battaile

Computational Materials Science and Engineering Department

Sandia National Laboratories

Dr. David McDowell

School of Materials Science and Engineering Georgia Institute of Technology

Date Approved: October 20, 2006 


\section{ACKNOWLEDGEMENTS}

I wish to thank my advisor Dr. Saxena for taking me on as a graduate student and then allowing me the opportunity to do a computationally based research project at Sandia National Laboratories. I also thank my Sandia mentor and friend Dr. Corbett Battaile for taking on a graduate student he had never met and had no prior modeling experience. With Dr. Battaile's guidance and patience, I learned how to write code and run JAS3D and without Dr. Battaile's insight, there is no way this project would have come this far. I'd also like to thank my committee members for their instruction: Dr. Mo Li, Dr. Tom Sanders, and Dr. David McDowell. A special thanks to Dr. David McDowell for answering many emails about crystal plasticity and other modeling related questions.

I am grateful to Dr. Michael Braginsky for the many rigorous discussions we had on mechanics. I'll never be great at solid mechanics but I am definitely better than I was. I am also thankful for Dr. Braginsky's help defining the scope of this project early on. I would also like to thank Dr. Doug Bammann for his input on kinematics and thermodynamics. Thank you Dr. Luke Brewer for the experimental data I used in this dissertation. I am very grateful to my manager Dr. Eliot Fang and Dr. Liz Holm for funding my Ph.D. research. I would also like to thank all those in organization 1814 and to Kip Findley, Alejandro Ibanez, and Shubhra Bansal for their friendship.

And finally, this dissertation would not have been possible without the support of my wife Shayna. She sacrificed many nights and Saturdays to take care of Zach so that I could finish this work. 


\section{TABLE OF CONTENTS}

Page

ACKNOWLEDGEMENTS

iii

LIST OF TABLES

ix

LIST OF FIGURES

$\mathrm{X}$

NOMENCLATURE

xvii

SUMMARY

xxix

CHAPTER 1: INTRODUCTION

CHAPTER 2: LITERATURE REVIEW

2.1 Dislocation Densities and Microstructure $\quad 8$

2.1.1 Forest and Glide Dislocations $\quad 8$

2.1.2 Geometrically Necessary and Statistically Stored Dislocations $\quad 10$

2.2 General Dislocation Density-Property Relationships 15

2.3 Experimental Observations of Length Scale Dependent Phenomena 18

$\begin{array}{ll}\text { 2.3.1 Grain Size Effects } & 18\end{array}$

$\begin{array}{ll}\text { 2.3.2 Indentation } & 20\end{array}$

2.3.3 Specimen Dimension Effects in Bending and Torsion 23

2.3.4 Specimen Dimension Effects in Tension and Compression 26

2.3.4.1 Free Standing Films 26

$\begin{array}{ll}\text { 2.3.4.2 Cylindrical Specimens } & 27\end{array}$

2.4 Numerical Modeling of Length Scale Phenomena 31

2.4.1 Modeling Length Scale Phenomena with Local Models 31

2.4.2 Modeling Length Scale Phenomena with Non-Local Models 33

2.4.2.1 Gradient Modeling 34 
2.4.2.1.1 Gradient Plasticity with Gradient State Variables 36

2.4.2.1.2 Plasticity with Gradient Dependent Evolution Equations $\quad 39$

2.4.2.2 Non-local Integral Modeling $\quad 45$

2.4.2.2.1 Non-Local Integral Elasticity Models 46

2.4.2.2.2 Non-Local Integral Plasticity Models 48

CHAPTER 3: CRYSTAL PLASTICITY THEORY 53

3.1 Kinematics 54

3.1.1 Single Crystal $F^{e} F^{P}$

3.1.2 Polycrystal $F^{e} F^{P}$

$\begin{array}{ll}3.2 \text { Thermodynamics } & 68\end{array}$

3.2.1 Local ISV Model $\quad 68$

3.2.2 Gradient ISV Models 71

3.2.3 ISV Models with Non-Local Integral Variables 74

$\begin{array}{ll}3.3 \text { Kinetics } & 76\end{array}$

3.3.1 Dislocation Motion 76

3.3.1.1 Rate-Independent Formulations 77

3.3.1.2 Rate-Dependent Formulations 77

3.3.2 Work Hardening 79

3.3.2.1 Microstructure Insensitive Work Hardening Models 79

3.3.2.2 Microstructure Sensitive Work Hardening Models 81

3.4 Constitutive Relations $\quad 83$

3.4.1 Hypo-elastic Plasticity 83

3.4.2 Hyper-elastic Plasticity 85

3.5 Numerical Integration $\quad 86$

3.5.1 Explicit Integration 86 
$\begin{array}{ll}\text { 3.5.2 Implicit Integration } & 87\end{array}$

3.6 Calculating Gradient Quantities in Crystal Plasticity 87

3.6.1 Gradient Quantities Determined by Derivatives of Shape Functions $\quad 88$

3.6.2 Gradient Quantities Approximated with Integrals 89

CHAPTER 4: IMPLEMENTATION OF THE LOCAL AND NON-LOCAL CRYSTAL PLASTICITY MODEL 92

4.1 Role of Crystal Plasticity Models within Jas3D 93

4.2 Local Crystal Plasticity Model 96

$\begin{array}{ll}\text { 4.2.1 Initialization } & 96\end{array}$

$\begin{array}{ll}\text { 4.2.2 Stress Update } & 98\end{array}$

$\begin{array}{ll}\text { 4.2.3 Hardening } & 101\end{array}$

4.2.4 Stability Check/Sub-incrementation $\quad 103$

$\begin{array}{ll}\text { 4.3 Non-Local Crystal Plasticity Model } & 107\end{array}$

$\begin{array}{ll}\text { 4.3.1 Initialization } & 109\end{array}$

$\begin{array}{ll}\text { 4.3.2 Hardening } & 113\end{array}$

CHAPTER 5: SENSITIVITY ANALYSIS OF THE NON-LOCAL INTEGRAL BASED GRADIENT APPROXIMATION 116

$\begin{array}{ll}\text { 5.1 Evaluation of Continuous Functions } & 118\end{array}$

5.1.1 The Effect of Integration Volume, Mesh Refinement, and $\Theta \quad 118$

$\begin{array}{ll}\text { 5.1.2 The Effect of a Non-Uniform Mesh } & 124\end{array}$

$\begin{array}{ll}\text { 5.1.3 The Effect of } \omega & 127\end{array}$

5.2 Evaluation of Step Functions 131

5.2.1 The Effect of $\Theta$ Function Type 133

5.2.2 The Effect of Integration Volume 135

$\begin{array}{ll}\text { 5.2.3 The Effect of } \omega \text { on } & 138\end{array}$

5.3 Convergence of Nearest Neighbor Integration Volumes and Large $\omega$ 's $\quad 140$ 
CHAPTER 6: APPLICATION OF THE LOCAL AND NON-LOCAL CRYSTAL PLASTICITY MODELS TO POLYCRYSTALLINE COPPER

6.1 Simulation Parameters

6.1.1 Determination of Grain Orientations

6.1.2 Elastic and Viscoplastic Parameters

6.1.3 Hardening Parameters

6.1.4 Determination of Non-Local Model Parameters

6.1.5 Polycrystalline Material Meshes

6.1.6 Boundary Conditions

6.1.6.1 Free Surface Boundary Condition \#1 (FSBC \#1)

6.1.6.2 Free Surface Boundary Condition \#2 (FSBC \#2)

6.1.7 Determination of Yield Strength and Grain Size

6.2 Effect of Polycrystal Kinematics

6.2.1 Macroscopic Response

6.2.2 Microscale Response

6.3 Grain Size Dependence of Yield Strength in Copper

6.4 The Effect of Simulation Parameters on the Hall-Petch Response

6.4.1 Effect of Grain Shape on the Hall-Petch Response

6.4.2 Effect of $\mathrm{C}$ and $\omega$ on the Hall-Petch Response

6.4.3 Effect of Mesh Refinement on the Hall-Petch Response

6.4.4 Effect of the $\Theta$ Function on the Hall-Petch Response

6.4.5 Effect of the Misorientation Distribution on the Hall-Petch Response

6.5 The Effect of Boundary Conditions on the Non-Local Model's $\sigma-\varepsilon$

Response

6.6 Material Length Scale

6.7 Model Limitations 
6.7.1 GB Height vs. Misorientation

6.7.2 Large Strain Response Predicted by the Non-Local Model

CHAPTER 7: INVESTIGATION OF INTRAGRAIN MISORIENTATIONS IN POLYCRYSTALLINE NICKEL

7.1 Experimental Methods

7.2 Experimental Results

7.3 Simulation Parameters

7.3.1 Initial Microstructure and Boundary Conditions

7.3.2 Elastic and Viscoplastic Parameters

7.3.3 Hardening and Non-Local Parameters

7.4 Simulation Results

CHAPTER 8: CONCLUSIONS

8.1 Polycrystal Kinematics

8.2 Non-Local Integral Approach

8.3 Macroscale Hall-Petch Results

8.4 Microscale Misorientation Results

APPENDIX B: KOCK'S COMPOSITE MODEL FOR POLYCRYSTALLINE MATERIALS

APPENDIX C: EXPERIMENTAL HALL-PETCH DATA

APPENDIX D: THE LOCAL INTRAGRAIN MISORIENTATION (LIMIS) AND AVERAGE MISORIENTATION (AMIS) CALCULATIONS 


\section{LIST OF TABLES}

Page

Table 4.1: Features of the Local and Non-local Crystal Plasticity Models 92

Table 4.2: Summary of Local Crystal Plasticity Model 102

Table 4.3: $\left\|\dot{\gamma}_{t+\Delta t}^{\alpha}\right\|^{\text {CRIT }}$ needed for stability in the crystal plasticity models 104

Table 4.4: Summary of Non-Local Crystal Plasticity Model 115

Table 6.1: Elastic and viscoplastic parameters used in copper simulations $\quad 146$

Table 6.2: Hardening parameters used in copper simulations $\quad 150$

Table 6.3: Non-local parameters used in copper simulations 152

Table 6.4: Best-fit Hall-Petch equations to simulation and experiment data 172

Table 6.5: Best-fit Hall-Petch equations for a polycrystal composed of square, $\begin{array}{ll}\text { hexagonal, and realistic shape grains } & 177\end{array}$

Table 6.6: Best-fit Hall-Petch equations for simulations with varying C values $\quad 178$

Table 6.7: Best-fit Hall-Petch equations for simulations with varying $\omega$ values $\quad 180$

Table 6.8: Best-fit Hall-Petch Equations for converged and non-converged

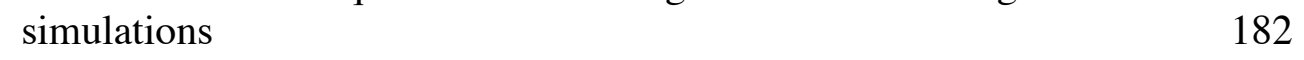

Table 6.9: Best-fit Hall-Petch equations to simulation results using a Gaussian $\Theta$ function $\quad 184$

Table 6.10: Best-fit Hall-Petch equations for the 3 different misorientation distributions

Table 7.1: Elastic and viscoplastic parameters used in nickel simulations $\quad 210$

Table 7.2: Hardening parameters used in nickel simulations 212

Table 7.3: Non-local parameters used in nickel simulations 212

Table C.1: Best-fit Hall-Petch equations to experimental Hall-Petch data for copper 


\section{LIST OF FIGURES}

Page

Figure 1.1: Polycrystalline metal microstructures

Figure 1.2: Predicted stress-strain curves from a local and non-local plasticity model for a polycrystal with an average grain size of $19 \mu \mathrm{m}$ and $38 \mu \mathrm{m}$

Figure 2.1: Forest and glide dislocations

Figure 2.2: Dislocations needed to accommodate a bending curvature (Arsenlis and Parks, 1999)

Figure 2.3: A burgers circuit around GNDs and SSDs

Figure 2.4: Non-uniform deformations resulting from the applied load (a,b,c,d) or the material's microstructure (e,f). (Fleck et al., 1994)

Figure 2.5: If each grain in the polycrystal shown at (a) deforms without the constraint of its neighbors, voids and overlaps appear as seen in (b). These voids and overlaps can be eliminated by GNDs as shown in (c) and (d). (Ashby, 1970)

Figure 2.6: Comparison of $\rho^{\mathrm{GND}}$ and $\rho^{\mathrm{SSD}}$. Single crystal data was taken from Basinski and Basinski (1966). Fleck et al. (1994) inferred the polycrystal data from stress-strain curves. In this figure $\lambda=\lambda^{\mathrm{GND}}$.

Figure 2.7: Dislocation density vs. flow stress. The straight lines in Figure 2.7 represent $\tau=C G b \sqrt{\rho^{F}}$ with $C=1$ and 0.5. (Mecking and Kocks, 1981)

Figure 2.8: Hall-Petch response of copper for at a number of different strains. (Hansen,1983)

Figure 2.9: The ISE in copper. (McElhaney et al., 1998)

Figure 2.10: Compressive (A) and Rotational (B) displacement fields under an indenter tip. The rotational plastic displacement field results in GND storage. (Stelmashenko et al., 1983)

Figure 2.11: Tension and torsion response of copper wires with diameters of $12 \mu \mathrm{m}$ to $170 \mu \mathrm{m}$. (Fleck et al., 1994)

Figure 2.12: Stress-strain response of a 1.0 and $0.2 \mu \mathrm{m}$ free-standing film (Espinosa et al., 2004) 
Figure 2.13: Stress-strain curves for the micron sized compression specimens (Uchic et al., 2004)

Figure 2.14: Effect of pillar diameter on flow stress (Greer et al., 2005)

Figure 2.15: The lattice mismatch at the grain boundary that determined the initial GND density (Evers et al., 2004b)

Figure 3.1: Single crystal kinematics

Figure 3.2: Single crystal kinematics applied to a polycrystal without initialization

Figure 3.3: Single crystal kinematics applied to a polycrystal with initialization

Figure 3.4: Polycrystal kinematics

Figure 3.5: An incompatible crystal in the natural configuration

Figure 3.6: 1D Bar Element

Figure 4.1: Relationship between the Finite Element Code and the Material Model. Quantities at the beginning of the time step have a subscript $t$ and quantities at the end of the time step $\Delta t$ have a subscript $t+\Delta t$

Figure 4.2: Illustration of sub-incrementation. A solid line indicates a stable time step, and a dashed line represents an unstable time step.

Figure 4.3: Stress-strain response of a single crystal meshed with between 7500 and 16,000 elements.

Figure 5.1: Function $\mathrm{y}=\mathrm{x}^{3}$ plotted over a grid of 11 elements each with a length of 1 . $\{\nabla \mathrm{x}\}$ was taken at the element designated by

Figure 5.2: The effects of integration volume, mesh refinement, and $\Theta$ on $\{\nabla \mathbf{x}\}$ of $y=x^{3}$. In the exponential attenuation function, $\omega=0.5$.

Figure 5.3: Function $\mathrm{y}=\mathrm{x}^{-0.5}$ between $\mathrm{x}=0$ and $\mathrm{x}=10$ plotted over a grid of 11 elements each with a length of $1 .\{\nabla \mathrm{x}\}$ was taken at the element designated by $\boldsymbol{\Delta} .122$

Figure 5.4: Function $\mathrm{y}=\mathrm{x}^{-0.5}$ between $\mathrm{x}=10$ and $\mathrm{x}=20$ plotted over a grid of 41 elements each with a length of $1 .\{\nabla \mathrm{x}\}$ was taken at the element designated by

Figure 5.5: The effects of integration volume, mesh refinement, and $\Theta$ on $\{\nabla x\}$ at $\mathrm{x}=5$ for $\mathrm{y}=\mathrm{x}^{-0.5}$. In the exponential attenuation function, $\omega=0.5$. 
Figure 5.6: The effects of integration volume, mesh refinement, and $\Theta$ on $\{\nabla x\}$ at $\mathrm{x}=10$ for $\mathrm{y}=\mathrm{x}^{-0.5}$. In the exponential attenuation function, $\omega=0.5$.

Figure 5.7: Function $\mathrm{y}=\mathrm{x}^{-0.5}$ between $\mathrm{x}=-2$ and $\mathrm{x}=2$ plotted over a grid with an average element size of $1 .\{\nabla \mathrm{x}\}$ was taken at the element designated by $\boldsymbol{\Lambda}$.

Figure 5.8: Function $\mathrm{y}=\mathrm{x}^{-0.5}$ between $\mathrm{x}=-2$ and $\mathrm{x}=2$ plotted over a grid with an average element size of $0.3 .\{\nabla \mathrm{x}\}$ was taken at the element designated by $\boldsymbol{\Lambda}$.

Figure 5.9: The effect of non-symmetric mesh on $\{\nabla x\}$ for $y=x^{3}$ at $x=0$. In the exponential attenuation function, $\omega=0.5$.

Figure 5.10: The attenuation function, $\Theta=\operatorname{Exp}\left(-\frac{|\xi|}{\omega}\right)$, plotted between $\xi=-5$ and $\xi=5$.

Figure 5.11: The attenuation function, $\Theta=\operatorname{Exp}\left(-\frac{\xi^{2}}{\omega^{2}}\right)$, plotted between $\xi=-5$ and $\xi=5$.

Figure 5.12: The effect of the $\omega$ in $\Theta=\operatorname{Exp}\left(-\frac{|\xi|}{\omega}\right)$ on $\{\nabla \mathrm{x}\}$ for $\mathrm{y}=\mathrm{x}^{3}$ at $\mathrm{x}=0$.

Figure 5.13: The effect of the $\omega$ in $\Theta=\operatorname{Exp}\left(-\frac{|\xi|}{\omega}\right)$ on $\{\nabla \mathrm{x}\}$ for $\mathrm{y}=\mathrm{x}^{3}$ at $\mathrm{x}=0$.

Figure 5.14: The step-function $y=\left\{\begin{array}{ll}4 & x<7.5 \\ 9 & x>7.5\end{array}\right\}$ between $x=1$ and $x=14$ plotted over a grid with an average element size of $1 .\{\nabla \mathrm{x}\}$ was taken at the element designated by

Figure 5.15: The effect of $\Theta$ on $\{\nabla x\}$ of a step-function

Figure 5.16: The gradient calculated with the non-local integral based approach and the shape function based approach.

Figure 5.17: The effect of integration volume on $\{\nabla x\}$ of a step function using $\Theta=1$. 
Figure 5.18: The effect of integration volume on $\{\nabla \mathrm{x}\}$ of a step function

$$
\text { using } \Theta=\operatorname{Exp}\left(-\frac{|\xi|}{\omega}\right)
$$

Figure 5.19: The effect of $\omega$ on $\{\nabla x\}$ of a step-function using $\Theta=\operatorname{Exp}\left(-\frac{|\xi|}{\omega}\right)$.

Figure 5.20: The effect of an $\mathrm{L}_{\text {cell }}$ that is too small for a given $\omega$.

Figure 5.21: An arbitrary function plotted over a uniform grid composed of 5 elements. $\{\nabla \mathbf{x}\}$ was taken at the element designated by $\mathbf{\Delta}$.

Figure 5.22: An arbitrary function plotted over a non-uniform grid composed of 5 elements. $\{\nabla \mathrm{x}\}$ was taken at the element designated by $\mathbf{\Delta}$.

Figure 6.1: 100 stress-strain curves for 100 different sets of orientations. The "average" stress-strain curve is in red.

Figure 6.2: Stress-strain curves from Fernandes and Viera (2000)

Figure 6.3: Stress-strain curves from Hansen (1979)

Figure 6.4: Polycrystalline meshes using idealized grain shapes

Figure 6.5: A polycrystalline mesh with 25 realistic grains

Figure 6.6: Displacement boundary conditions used in all simulations

Figure 6.7: The integration volume for an element at the boundary using FSBC \#1. Non-local quantities are evaluated at the element with $a \bullet$.

Figure 6.8: The integration volume for an element at the boundary using FSBC \#2. Non-local quantities are evaluated at the element with a $\bullet$.

Figure 6.9: Predicted stress-strain response of polycrystalline copper with grain sizes between $14 \mu \mathrm{m}$ and $77 \mu \mathrm{m}$ from a non-local model without $\{\tilde{\mathbf{G}}\}$.

Figure 6.10: Experimental stress-strain curves for a polycrystalline material with different grain sizes. (Fleck et al., 1994)

Figure 6.11: Predicted stress-strain response of polycrystalline copper with grain sizes between $14 \mu \mathrm{m}$ and $77 \mu \mathrm{m}$ from a non-local model with $\{\tilde{\mathbf{G}}\}$. 
Figure 6.12: A Hall-Petch plot comparing experimental results to simulation results from the non-local mode with and without $\{\tilde{\mathbf{G}}\}$.

Figure 6.13: $\bar{\tau}^{\mathrm{CSS}}$ at $\varepsilon=0$ for a non-local model without $\{\tilde{\mathbf{G}}\}$. Note that the $14 \mu \mathrm{m}$ picture has an area that is roughly $1 / 30^{\text {th }}$ of the $77 \mu \mathrm{m}$ picture.

Figure 6.14: $\bar{\tau}^{\mathrm{CSS}}$ at $\varepsilon=0.5 \%$ for a non-local model without $\{\tilde{\mathbf{G}}\}$. Note that the $14 \mu \mathrm{m}$ picture has an area that is roughly $1 / 30^{\text {th }}$ of the $77 \mu \mathrm{m}$ picture.

Figure 6.15: $\bar{\tau}^{\mathrm{CSS}}$ at $\varepsilon=0$ for a non-local model with $\{\tilde{\mathbf{G}}\}$. Note that the $14 \mu \mathrm{m}$ picture has an area that is roughly $1 / 30^{\text {th }}$ of the $77 \mu \mathrm{m}$ picture.

Figure 6.16: $\bar{\tau}^{\mathrm{CSS}}$ at $\varepsilon=0.5 \%$ for a non-local model with $\{\tilde{\mathbf{G}}\}$. Note that the $14 \mu \mathrm{m}$ picture has an area that is roughly $1 / 30^{\text {th }}$ of the $77 \mu \mathrm{m}$ picture.

Figure 6.17: The change in $\bar{\tau}^{\mathrm{CSS}}$ away from the grain boundary in both the $14 \mu \mathrm{m}$ and $77 \mu \mathrm{m}$ grain size polycrystal

Figure 6.18: Hall-Petch plot comparing experimental results from Fernandes and Viera (2000) to simulation results. The local model result is shown as a dashed line sine this result is grain size independent.

Figure 6.19: Hall-Petch plot comparing experimental results from Hansen (1983) to simulation results. The local model result is shown as a dashed line sine this result is grain size independent.

Figure 6.20: The simulated Hall-Petch response for a polycrystal made up of square, hexagon and realistic shaped grains.

Figure 6.21: The dependence of the simulated Hall-Petch response on C

Figure 6.22: The dependence of the simulated Hall-Petch response on $\omega$

Figure 6.23: The effect of mesh refinement on the Hall-Petch response

Figure 6.24: Simulated Hall-Petch curves from a non-local model using a Gaussian $\Theta$ function

Figure 6.25: Comparison of misorientation distributions with the MacKenzie distribution

Figure 6.26: The effect of misorientation distribution on the Hall-Petch response 
Figure 6.27: A comparison of stress-strain curve generated with different boundary conditions

Figure 6.28: A hardening profile generated by FSBC \#1 and FSBC \#2 for a grain boundary that intersects the specimen

Figure 6.29: The definition of GB layer width and GB strength

Figure 6.30: The effect of changing $\rho_{\mathrm{t}=0}^{\mathrm{SSD}}$ with $\mathrm{C}$ on the material GB layer width

Figure 6.31: The overall effect of $\mathrm{C}$ on the GB layer width and GB strength

Figure 6.32: The effect of $\mathrm{C}$ on the GB layer width and GB strength

Figure 6.33: The effect of $\mathrm{C}$ and $\omega$ on the GB layer width

Figure 6.34: The effect of $\mathrm{C}$ and $\omega$ on the GB strength

Figure 6.35: A low angle tilt boundary composed of edge dislocations

Figure 6.36: How the change in misorientation leads to a change in GB strength

Figure 6.37: Misorientation angle vs. GB strength

Figure 6.38: Stress-strain results from the local and non-local models out to $17 \%$ strain

Figure 7.1: An inverse pole figure map along the loading $(\mathrm{X})$ axis

Figure 7.2: Composite stress-strain curve for the nickel tensile specimen

Figure 7.3: Inverse pole figure maps at $1 \%, 5 \%$, and $10 \%$ strain

Figure 7.4: The initial meshed microstructure and the applied displacement boundary conditions

Figure 7.5: Local and non-local model fit to the experimental stress-strain curve

Figure 7.6: Local intragrain misorientation maps at $1 \%$ strain

Figure 7.7: Local intragrain misorientation maps at $5 \%$ strain

Figure 7.8: Local intragrain misorientation maps at $10 \%$ strain

Figure 7.9: Local LIMIS results at 10\% strain 
Figure 7.11: Comparison of experimental, local simulation, and non-local simulation AMIS results

Figure B.1: A square grain composed of a hardened grain boundary region, $\mathrm{A}_{\mathrm{GB}}$, and softer grain core, $\mathrm{A}_{\mathrm{I}}$

Figure B.2: A hexagon shaped grain composed of a hardened grain boundary region, $A_{G B}$, and softer grain core, $A_{I}$

Figure C.1: Experimental Hall-Petch data for copper

Figure D.1: Square shaped composed of 9 elements 


\section{NOMENCLATURE}

\begin{tabular}{|c|c|}
\hline \multicolumn{2}{|c|}{ Abbreviations } \\
\hline \multicolumn{2}{|c|}{$1 \mathrm{D}$} \\
\hline 2D & Two Dimensional \\
\hline $3 \mathrm{D}$ & Three Dimensional \\
\hline AMIS & Average Misorientation \\
\hline EBSD & Electron Backscatter Diffraction \\
\hline FCC & Face centered cubic \\
\hline FEM & Finite Element Method \\
\hline FIB & Focused Ion Beam \\
\hline FSBC & Free Surface Boundary Conditions \\
\hline GB & Grain Boundary \\
\hline GND & Geometrically Necessary Dislocation \\
\hline IPF & Inverse Pole Figure \\
\hline ISE & Indenter Size Effect \\
\hline ISV & Internal State Variable \\
\hline LIMIS & Local Intragrain Misorientation \\
\hline MEMS & Microelectromechanical Systems \\
\hline MSG & Mechanism based Strain Gradient \\
\hline PLC & Portevin-Le Chatelier \\
\hline PBC & Periodic Boundary Conditions \\
\hline PSB & Persistent Slip Band \\
\hline
\end{tabular}


SSD

TEM

\section{Symbols - Greek}

$\begin{array}{ll}\{\nabla \mathrm{x}\} & \text { Non-local integral based gradient of } \mathrm{x} \\ \alpha, \beta & \text { Slip system indices } \\ \chi & \text { Rotational part of } \boldsymbol{\varepsilon} \text { (curvature tensor) } \\ \chi^{\mathrm{P}} & \text { Plastic rotational part of } \chi \\ \chi^{\mathrm{eff}} & \text { Effective plastic rotation } \\ \Delta^{\alpha} & \text { Lattice mismatch in grain } \alpha \\ \Delta \mathrm{t} & \text { Time step } \\ \Delta \mathrm{t}_{\mathrm{crit}} & \text { Critical time step } \\ \delta_{\mathrm{ij}} & \text { Kronecker delta } \\ \boldsymbol{\varepsilon} & \text { Strain tensor } \\ \boldsymbol{\varepsilon}^{\mathrm{e}} & \text { Elastic strain tensor } \\ \boldsymbol{\varepsilon}^{\mathrm{P}} & \text { Plastic strain tensor } \\ \left\{\boldsymbol{\varepsilon}^{\mathrm{e}}\right\} & \text { Non-local integral average of elastic strain } \\ \left\{\boldsymbol{\varepsilon}^{\mathrm{P}}\right\} & \text { Non-local integral average of plastic strain } \\ \widehat{\varepsilon} & \text { Microscale strain tensor } \\ \widehat{\mathcal{\varepsilon}}^{\mathrm{efff}} & \text { Euler angles } \\ \phi_{1}, \phi_{2}, \phi_{3} & \boldsymbol{\varepsilon}\end{array}$

Statistically Stored Dislocation

Transmission Electron Microscope 


\begin{tabular}{|c|c|}
\hline$\Lambda^{\text {eff }}$ & Effective $\Lambda$ \\
\hline$\Gamma$ & Angle of an indenter tip \\
\hline$\gamma$ & Slip tensor \\
\hline$\hat{\gamma}$ & Slip tensor in the intermediate configuration \\
\hline$\gamma$ & Slip or plastic shear strain \\
\hline$\{\gamma\}$ & Non-local integral average of plastic slip \\
\hline$\dot{\gamma}^{\alpha}$ & Slip rate on slip system $\alpha$ \\
\hline$\dot{\gamma}_{0}$ & Reference slip rate \\
\hline$\dot{\gamma}^{*}$ & Representative shearing rate \\
\hline$\|\dot{\gamma}\|$ & Slip rate magnitude \\
\hline$\|\dot{\gamma}\|^{\text {Crit }}$ & Critical slip rate magnitude \\
\hline$\eta$ & Strain gradient tensor \\
\hline$\eta^{\text {eff }}$ & Effective strain gradient \\
\hline$\kappa$ & Hardening/softening function in flow plasticity theories \\
\hline$\{\kappa\}$ & Non-local integral average of the hardening/softening parameter \\
\hline$\lambda$ & Material length scale \\
\hline$\lambda^{\mathrm{GND}}$ & GND length scale \\
\hline$\lambda^{\mathrm{SSD}}$ & SSD length scale \\
\hline$v$ & Poisson's ratio \\
\hline$\Omega$ & Total spin rate tensor \\
\hline
\end{tabular}




\begin{tabular}{|c|c|}
\hline$\Omega^{\mathrm{e}}$ & Elastic spin rate tensor \\
\hline $\boldsymbol{\theta}$ & Rotation vector associated with a displacement field \\
\hline$\theta$ & Misorientation angle \\
\hline $\bar{\theta}$ & Average Misorientation angle \\
\hline$\Theta$ & Attenuation Function \\
\hline$\Pi$ & An arbitrary gradient variable associated with microstructure \\
\hline$\rho_{\text {DEN }}$ & Density \\
\hline$\rho^{\mathrm{F}}$ & Forest dislocation density \\
\hline$\rho^{\mathrm{G}}$ & Glide dislocation density \\
\hline$\rho^{\mathrm{GND}}$ & Geometrically necessary dislocation density \\
\hline$\rho^{I}$ & Immobile dislocation density \\
\hline$\rho^{\mathrm{M}}$ & Mobile dislocation density \\
\hline$\rho^{\mathrm{SA}}$ & Dislocation density surrounded by a cloud of solute atoms \\
\hline$\rho^{\mathrm{SSD}}$ & Statistically stored dislocation density \\
\hline$\langle\rho\rangle$ & Average dislocation density \\
\hline$[\rho]$ & Dislocation density matrix \\
\hline$\sigma$ & Cauchy stress tensor \\
\hline$\sigma^{\prime}$ & Deviatoric Cauchy stress tensor \\
\hline$\hat{\sigma}^{\mathrm{PK} 2}$ & $2^{\text {nd }}$ Piola-Kirchhoff stress in the intermediate configuration \\
\hline$\dot{\sigma}$ & Time derivative of Cauchy stress tensor \\
\hline $\begin{array}{c}\nabla \\
\sigma^{*}\end{array}$ & Objective Cauchy stress rate \\
\hline
\end{tabular}




\begin{tabular}{|c|c|}
\hline$\sigma^{* J}$ & $\begin{array}{l}\text { Jaumann objective Cauchy stress rate } \mathrm{d} \\
\text { rotates with the lattice }\end{array}$ \\
\hline$\stackrel{\nabla}{\sigma^{J}}$ & $\begin{array}{l}\text { Jaumann objective Cauchy stress rate } d \\
\text { rotates with the material }\end{array}$ \\
\hline$\hat{\sigma}$ & Microscale Cauchy stress tensor \\
\hline$\sigma^{\mathrm{eff}}$ & Effective Cauchy stress \\
\hline$\hat{\sigma}^{\text {eff }}$ & Effective $\hat{\boldsymbol{\sigma}}$ \\
\hline$\sigma_{\mathrm{I}}$ & Flow stress of grain interior region \\
\hline$\sigma_{\mathrm{GB}}$ & Flow stress of grain boundary region \\
\hline$\sigma_{\mathrm{y}}$ & Yield strength \\
\hline$\sigma_{0}$ & Initial yield strength \\
\hline$\tau^{\alpha}$ & Resolved shear stress on slip system $\alpha$ \\
\hline$\tau_{0}$ & Initial shear stress \\
\hline$\tau_{\text {sat }}$ & Saturation shear stress \\
\hline$\tau^{\mathrm{b}}$ & Total back stress \\
\hline$\tau^{\mathrm{CSS}}$ & Critical shear stress \\
\hline$\tau_{\mathrm{e}}$ & Back stress due to edge dislocations \\
\hline$\tau_{\mathrm{s}}$ & Back stress due to screw dislocations \\
\hline$\tau^{\mathrm{LIM}}$ & Length scale independent flow stress \\
\hline $\bar{\tau}^{\mathrm{CSS}}$ & Average flow stress \\
\hline$\dot{\tau}^{*}$ & Effective grain hardness \\
\hline$\varpi$ & Indentation depth \\
\hline
\end{tabular}


$\omega$

$\psi$

$\xi$

$\|\xi\|$

$\zeta$

$\zeta$

\section{Symbols - Alphanumeric}

$$
\#_{\mathrm{R} 1}, \#_{\mathrm{R} 2}, \#_{\mathrm{R} 3}
$$

\#N

$\mathbf{a}$

A

$\mathrm{A}^{\alpha \zeta}$

$\mathrm{A}_{\mathrm{I}}$

$\mathrm{A}_{\mathrm{GB}}$

b

$\hat{\mathbf{b}}, \hat{\mathbf{b}}^{\mathbf{P}}, \hat{\mathbf{b}}^{\mathrm{e}}$

b

[B]

c

C

$\mathrm{C}_{1}, \mathrm{C}_{2}$

$\mathrm{c}_{\mathrm{AB} 0}$ type $\zeta$
Length parameter in attenuation functions

Helmholtz free energy per unit mass

Position vector in local coordinates

Magnitude of vector $\xi$

Dislocation tangent vector

Dislocation type index

Random numbers

Number of neighbors

Arbitrary vector

Ratio between normal stress and hardness

Strength of dislocation junctions on slip plane $\alpha$ for dislocation

Area fraction of grain interior region

Area fraction of grain boundary region

Burgers vector

Burgers vector in the intermediate configuration

Magnitude of burgers vector

Shape function derivative matrix

Couple vector

Material constant in Taylor's equation

Material constant in Zikery-Kao model

Material constant in the Acharya-Beaudoin model 


\begin{tabular}{|c|c|}
\hline $\mathrm{c}_{\mathrm{AN} 0^{-}} \mathrm{c}_{\mathrm{AN} 6}$ & Material constants in the Ananthakrishna model \\
\hline $\mathrm{c}_{\mathrm{BW} 1}$ & Material constant in the Bassani-Wu model \\
\hline $\mathrm{c}_{\mathrm{EV} 1}$ & Material constant in the Evers model \\
\hline $\mathrm{c}_{\mathrm{FH} 1}-\mathrm{c}_{\mathrm{FH} 3}$ & Material constants in the Fleck-Hutchinson model \\
\hline $\mathrm{c}_{\mathrm{HU} 1}$ & Material constant in the Hutchinson model \\
\hline $\mathrm{c}_{\mathrm{KM} 1}, \mathrm{c}_{\mathrm{KM} 2}$ & Material constants in the Kocks-Mecking model \\
\hline $\mathrm{c}_{\mathrm{MD} 1}$ & Material constant in the Mathur-Dawson model \\
\hline $\mathrm{c}_{\mathrm{MU} 1}$ & Material constant in the Mughrabi model \\
\hline $\mathrm{c}_{\mathrm{TH} 1}, \mathrm{c}_{\mathrm{TH} 2}$ & Material constants in the Thompson model \\
\hline $\mathrm{c}_{\mathrm{WA} 1}$ & Material constant in the Walgraef-Aifantis model \\
\hline $\mathrm{c}_{\mathrm{ZK} 1}-\mathrm{c}_{\mathrm{ZK} 4}$ & Material constants in the Zikery-Kao model \\
\hline D & Total deformation rate tensor \\
\hline $\mathbf{D}^{\mathbf{P}}$ & Plastic deformation rate tensor \\
\hline $\mathbf{D}^{\mathrm{e}}$ & Elastic deformation rate tensor \\
\hline $\mathrm{d} \mathbf{x}$ & Differential line segment in the current configuration \\
\hline $\mathrm{d} \mathbf{X}$ & Differential line segment in the reference configuration \\
\hline $\mathrm{d} \hat{\mathbf{X}}$ & Differential line segment in the intermediate configuration \\
\hline d & Grain size \\
\hline Dim & Number of spatial dimensions $(1,2$, or 3$)$ \\
\hline$d_{e}^{\zeta \alpha}$ & Describes the way edge dislocation type $\zeta$ relates to slip system $\alpha$ \\
\hline$d_{s}^{\zeta \alpha}$ & Describes the way screw dislocation type $\zeta$ relates to slip system $\alpha$ \\
\hline $\mathrm{D}_{\mathrm{WA}}^{\mathrm{I}}$ & $\begin{array}{l}\text { Immobile dislocation diffusion constant in the Walgraef-Aifantis } \\
\text { model }\end{array}$ \\
\hline
\end{tabular}




\begin{tabular}{|c|c|}
\hline $\mathrm{D}_{\mathrm{wA}}^{\mathrm{M}}$ & $\begin{array}{l}\text { Mobile dislocation diffusion constant in the Walgraef-Aifanti } \\
\text { model }\end{array}$ \\
\hline $\mathrm{D}_{\mathrm{SE}}$ & Dislocation diffusion constant in the Sluys-Estrin model \\
\hline [d] & Displacement matrix \\
\hline$\hat{\mathbf{E}}^{\mathrm{e}}$ & Green elastic strain tensor in the intermediate configuration \\
\hline E & Internal energy per unit mass \\
\hline$e_{i j k}$ & Permutation symbol \\
\hline $\mathbf{F}$ & Total deformation gradient \\
\hline $\mathbf{F}^{\mathrm{e}}$ & Elastic deformation gradient \\
\hline $\mathbf{F}^{\mathbf{P}}$ & Plastic deformation gradient \\
\hline $\mathbf{F}^{\mathrm{I}}$ & Meso-scale incompatibility tensor \\
\hline $\mathbf{F}^{\theta}$ & Thermal deformation gradient tensor \\
\hline $\mathbf{F}^{\mathrm{d}}$ & Plastic deformation gradient associated with defects \\
\hline $\mathbf{F}^{\mathrm{m}}$ & Plastic deformation gradient associated with matrix \\
\hline $\mathbf{F}_{\mathbf{R}}$ & Deformation gradient composed of only a rotation \\
\hline $\mathbf{F}_{\mathrm{h}}$ & Preliminary deformation gradient tensor \\
\hline $\mathbf{F}_{\mathbf{h}}^{\mathrm{e}}$ & Preliminary elastic deformation gradient tensor \\
\hline $\mathbf{F}_{\mathbf{h}}^{\mathbf{P}}$ & Preliminary plastic deformation gradient tensor \\
\hline$\left\{\mathbf{F}^{\mathrm{I}}\right\}$ & Non-local integral average meso-incompatibility tensor \\
\hline$\left\{\mathbf{F}^{\mathbf{P}}\right\}$ & Non-local integral average plastic deformation gradient \\
\hline$f$ & Yield function in flow plasticity theories \\
\hline$f(\mathrm{x})$ & Arbitrary function \\
\hline $\mathrm{f}^{\alpha}$ & Level function for slip system $\alpha$ \\
\hline
\end{tabular}


g

$\hat{\mathbf{G}}, \hat{\mathbf{G}}^{\mathbf{P}}, \hat{\mathbf{G}}^{\mathbf{e}}$

$\hat{\mathbf{G}}^{\text {Tot }}$

$\tilde{\mathbf{G}}$

$\{\tilde{\mathbf{G}}\},\{\hat{\mathbf{G}}\}$

$g^{\text {eff }}$

G

$\mathrm{G}_{110}$

$\mathrm{H}$

$\mathrm{h}$

$h^{\alpha \beta}$

$\mathrm{h}_{0}, \mathrm{~h}_{\mathrm{s}}, \mathrm{f}_{\alpha \beta}$

j

$\mathbf{J}^{\mathrm{e}}$

$\mathbf{J}^{\mathrm{P}}$

$\mathbf{J}^{\mathbf{F}_{\mathbf{h}}^{\mathbf{e}}}$

$\mathbf{J}^{\mathbf{F}_{\mathbf{h}}^{\mathbf{P}}}$

$\mathbf{J}^{\mathbf{R}_{\mathrm{h}}^{\mathrm{e}}}$

$\mathrm{k}$

$\mathbf{L}$

$\hat{\mathbf{L}}^{\mathbf{P}}$

$\underset{\sim}{L}$
Nye's Dislocation tensor

Dislocation tensors in the intermediate configuration

Total dislocation tensor in the intermediate configuration

Dislocation tensor in the natural configuration

Non-local integral based dislocation tensors

Effective $\mathbf{g}$

Elastic shear modulus

Elastic shear modulus in the 110 direction

Hardness

Entropy flux

Matrix containing hardening moduli

Various hardening parameters

Misorientation tensor

Determinant of $\mathbf{F}^{\mathbf{e}}$

Determinant of $\mathbf{F}^{\mathbf{P}}$

Determinant of $\mathbf{F}_{\mathbf{h}}^{\mathbf{e}}$

Determinant of $\mathbf{F}_{\mathbf{h}}^{\mathbf{P}}$

Determinant of $\mathbf{R}_{\mathbf{h}}^{\mathbf{e}}$

Hall-Petch slope, Boltzmann's constant

Velocity gradient

Plastic velocity gradient in the intermediate configuration

Fourth order elasticity tensor 


\begin{tabular}{|c|c|}
\hline$\underline{\sim}_{\mathrm{S} \text {-xtal }}$ & Elasticity tensor in the single crystal configuration \\
\hline$L_{11}, L_{12}, L_{44}$ & Elastic constants from the $6 \times 6$ elasticity matrix \\
\hline $\mathrm{L}$ & Dislocation spacing \\
\hline 1 & Average dislocation mean free path \\
\hline$\ell$ & Length \\
\hline Len & Element length \\
\hline $\mathrm{L}_{\text {cell }}$ & Integration length \\
\hline $\mathbf{m}^{\alpha}$ & Normal vector to slip plane $\alpha$ in the current configuration \\
\hline $\mathbf{M}^{\alpha}$ & Normal vector to slip plane $\alpha$ in the reference configuration \\
\hline$\hat{\mathbf{m}}^{\alpha}$ & Normal vector to slip plane $\alpha$ in the intermediate configuration \\
\hline $\mathbf{m}_{\text {xtal }}^{\alpha}$ & Normal vector to slip plane a in the single crystal configuration \\
\hline $\mathrm{m}$ & Rate sensitivity exponent \\
\hline $\mathbf{n}$ & Normal vector in the current configuration \\
\hline $\mathbf{N}$ & Normal vector in the current configuration \\
\hline$\hat{\mathbf{N}}$ & Normal vector in the intermediate configuration \\
\hline$\overline{\mathbf{n}}$ & Skew-symmetric tensor in the Acharya-Beaudoin model \\
\hline $\mathrm{N}_{1}, \mathrm{~N}_{2}$ & FEM Shape functions \\
\hline $\mathrm{n}$ & Hall-Petch exponent \\
\hline $\mathbf{P}$ & Schmid orientation tensor \\
\hline $\mathbf{q}$ & Heat flux vector \\
\hline q & Latent Hardening parameter \\
\hline $\mathrm{Q}_{\text {slip }}$ & $\begin{array}{l}\text { Activation energy required to overcome obstacles to dislocation } \\
\text { motion }\end{array}$ \\
\hline
\end{tabular}




\begin{tabular}{|c|c|}
\hline $\mathbf{R}, \mathbf{R}^{\text {Base }}$ & Rotation tensor \\
\hline $\mathbf{R}^{\mathrm{e}}$ & Elastic rotation tensor \\
\hline $\mathbf{R}_{\mathrm{h}}^{\mathrm{e}}$ & Preliminary elastic rotation tensor \\
\hline $\mathbf{R}^{\text {sym }}$ & Symmetrically equivalent rotation tensor \\
\hline $\mathbf{R}^{\text {Lat }}$ & Updated (current) lattice orientations \\
\hline $\mathbf{R}^{\text {Orient }}$ & Rotation tensor that represents a grain orientation \\
\hline $\mathrm{r}, \mathbf{r}$ & Radius \\
\hline $\mathbf{s}^{\alpha}$ & Slip direction vector $\alpha$ in the current configuration \\
\hline $\mathbf{S}^{\alpha}$ & Slip direction vector $\alpha$ in the reference configuration \\
\hline$\hat{\mathbf{s}}^{\alpha}$ & Slip direction vector $\alpha$ in the intermediate configuration \\
\hline $\mathbf{s}_{\text {xtal }}^{\alpha}$ & Slip direction vector $\alpha$ in the single crystal configuration \\
\hline $\mathrm{S}$ & Entropy \\
\hline $\mathbf{t}$ & Traction vector \\
\hline $\mathrm{T}$ & Temperature \\
\hline $\mathbf{u}$ & Displacement field \\
\hline $\mathbf{U}_{\mathbf{R}}$ & Right stretch tensor in $\mathbf{F}_{\mathbf{R}}$ \\
\hline $\mathbf{U}_{\mathrm{h}}^{\mathrm{e}}$ & Preliminary elastic stretch tensor \\
\hline $\mathbf{V}_{\mathbf{R}}$ & Left stretch tensor in $\mathbf{F}_{\mathbf{R}}$ \\
\hline $\mathrm{V}_{\text {cell }}$ & Integration volume \\
\hline vol & Element volume \\
\hline $\begin{array}{l}\mathbf{v} \\
2\end{array}$ & $2^{\text {nd }}$ order higher order stress tensor \\
\hline
\end{tabular}




$\begin{array}{ll}\mathbf{v}_{2}^{\prime} & \text { Deviatoric part of the } \mathbf{v}_{2} \\ \mathbf{v}_{3} & 3^{\text {rd }} \text { order higher order stress tensor } \\ \mathbf{v}_{3}^{\prime} & \text { Deviatoric part of the } \mathbf{v}_{3} \\ \mathrm{v} & \text { Dislocation velocity } \\ \mathrm{v}_{0} & \text { Reference dislocation velocity } \\ \mathrm{v}^{\mathrm{M}} & \text { Mobile dislocation velocity } \\ \mathrm{v}^{\mathrm{I}} & \text { Immobile dislocation velocity } \\ \mathrm{v}^{\mathrm{SA}} & \text { Velocity of dislocation surrounded by solute atoms } \\ \mathrm{w} & \text { Stored elastic energy } \\ \mathrm{w}_{\mathrm{GB}} & \text { Grain boundary width } \\ \mathbf{x} & \text { Position vector in global coordinates } \\ \mathrm{y}_{\mathrm{c}} & \text { Critical annihilation length }\end{array}$




\section{SUMMARY}

It has been shown over the years that the mechanical response of polycrystalline metals depends on the metal's microstructure. One such example is the dependence of yield strength on grain size, other wise known as the Hall-Petch effect. Many conventional continuum approaches to solid mechanics do not address the sensitivity of deformation to microstructural features like grain boundaries, and are therefore unable to capture much of the experimentally observed behavior of polycrystal deformation.

In this work, a crystal plasticity model is developed that predicts a dependence of yield strength on grain size without grain size explicitly entering into the constitutive equations. The grain size dependence in the model is the result of non-local effects of geometrically necessary dislocations (GNDs). GNDs harden both the material at a point and the material in a non-local neighborhood. Numerically, these non-local affects are captured using a non-local integral approach rather than a conventional gradient approach. The conventional $\mathbf{F}^{\mathbf{e}} \mathbf{F}^{\mathbf{p}}$ kinematics for single crystals have been augmented based on a geometric argument that accounts for the grain orientations in a polycrystal. The augmented kinematics allows an initial GND state at grain boundaries and an evolving GND state due to sub-grain formation within the grain to be determined in a consistent manner.

The expanded kinematics and the non-local crystal plasticity model are used to simulate the tensile behavior in copper polycrystals with different grain sizes ranging from $14 \mu \mathrm{m}$ to $244 \mu \mathrm{m}$. The simulation results show a grain size dependence on the polycrystal's yield strength, which are qualitatively in good agreement with the 
experimental data. However, the Hall-Petch exponent predicted by the simulations is more like $\mathrm{d}^{-1}$ rather than $\mathrm{d}^{-0.5}$. The effects of different simulation parameters including grain shape and misorientation distribution did not greatly affect the Hall-Petch exponent. The simulation results indicate that the Hall-Petch exponent is sensitive to the grain boundary strength: the Hall-Petch exponent decreases as the grain boundary height decreases.

The intragrain misorientations predicted by the non-local model were compared with experiments on polycrystalline nickel. Experimentally, the intragrain misorientations were tracked by electron back scatter diffraction (EBSD) at 0\%, 1\%, 5\%, and $10 \%$ strain from the same location. On average, the simulation results predicted enough misorientation throughout the sample. However, the model did not correctly predict the spatial distribution of the intragrain misorientation. 


\section{CHAPTER 1}

\section{INTRODUCTION}

For many years experiments have repeatedly shown that mechanical response of metallic materials depends on the microstructure and the associated length scales. Microstructural length scales such as grain size and sub-grain size are known to affect the yield strength and the flow stress, respectively. Similarly, in precipitate hardened materials, length parameters such as precipitate size and spacing determine the yield and ultimate tensile strength, as well as the fracture toughness and fatigue resistance. For example, decreasing the grain size from $400 \mu \mathrm{m}$ (Hansen, 1983) to 100nm (Cheng et al., 2003) increases the yield strength significantly, a trend commonly known as the HallPetch effect. In precipitate hardened materials, inter-precipitate spacing as small as 1.4 nm has been shown to systematically increase the yield strength (Follstaedt et al., 2003).

Experiments have also shown that specimen dimensions can influence the mechanical properties of single crystal and polycrystal metallic materials, particularly when the specimen dimensions become comparable to the length scale of the controlling microstructural feature. Brenner (1956) found that as the single crystal whisker diameter decreased from $15 \mu \mathrm{m}$ to $1.2 \mu \mathrm{m}$, the strength (maximum stress) of the whisker increased. Fleck et al. (1994) observed an increase in the work hardening during torsion of polycrystalline copper wires as the diameter of the wire decreased from $170 \mu \mathrm{m}$ to $12 \mu \mathrm{m}$. Stolken and Evans (1998) reported a similar observation for the bending response of polycrystalline nickel foils with a thickness between $50 \mu \mathrm{m}$ and $12.5 \mu \mathrm{m}$. 
The large quantity of experimental data demonstrating the effect of specimen dimension on the strength of polycrystalline materials should be analyzed carefully. Using polycrystalline materials to study the effect of specimen dimension may convolute the effects due to grain size (or other microstrucutural length scale effects) and the effects due to specimen dimension. This research seeks to computationally isolate the effects of grain size in polycrystalline materials leading to a better understanding of the Hall-Petch relationship.

While experiments clearly show that microstructural length scales between hundreds of microns to the level of few microns affect the mechanical response, modeling these phenomena as a continuum covering the entire range has been a challenge. Local internal state variable (ISV) plasticity models ${ }^{1}$ are effective tools when they are applied to large structures (like bridges, aircraft wings, etc.) where material length scales are naturally homogenized. However local models that do not attempt to link element size to some physical scale are not well suited to model structures in which material length scales dominate the macroscopic response because the local model has no internal length scale. The need to capture length scale phenomena with continuum plasticity models has led to the development of non-local plasticity models².

To illustrate the need for non-local plasticity models, two polycrystalline microstructures, shown in Figure 1.1, were simulated using both a local and non-local ${ }^{3}$ crystal plasticity model. The resulting stress-strain curves predicted by the local and nonlocal model for both microstructures are shown in Figure 1.2.

\footnotetext{
${ }^{1}$ In local models, the state of a point depends on variables at that point only.

${ }^{2}$ In non-local models, the state of a point depends on variables at points in a surrounding area.

${ }^{3}$ This non-local model is described in Chapter 4.
} 


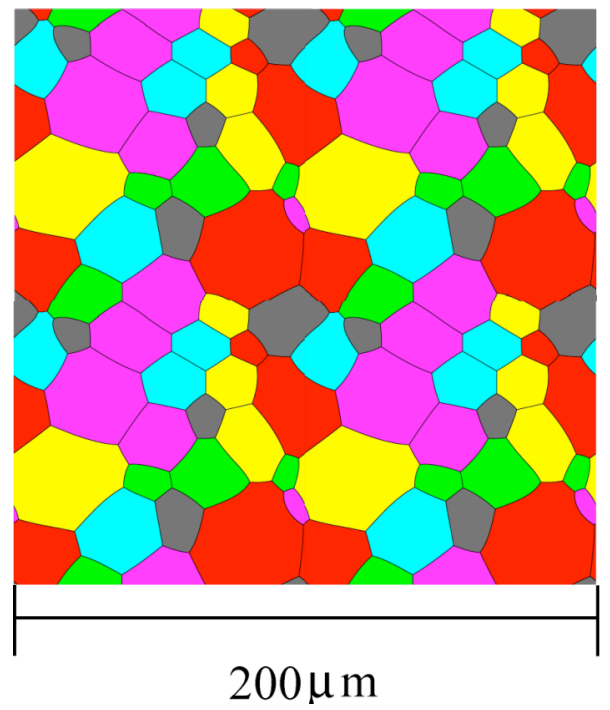

(a) Average Grain size $=19 \mu \mathrm{m}$

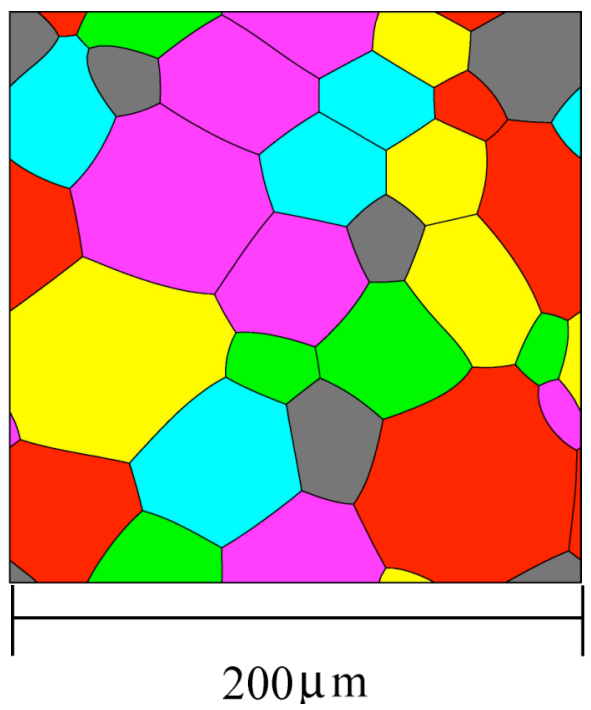

(b) Average Grain size $=38 \mu \mathrm{m}$

Figure 1.1 - Polycrystalline metal microstructures

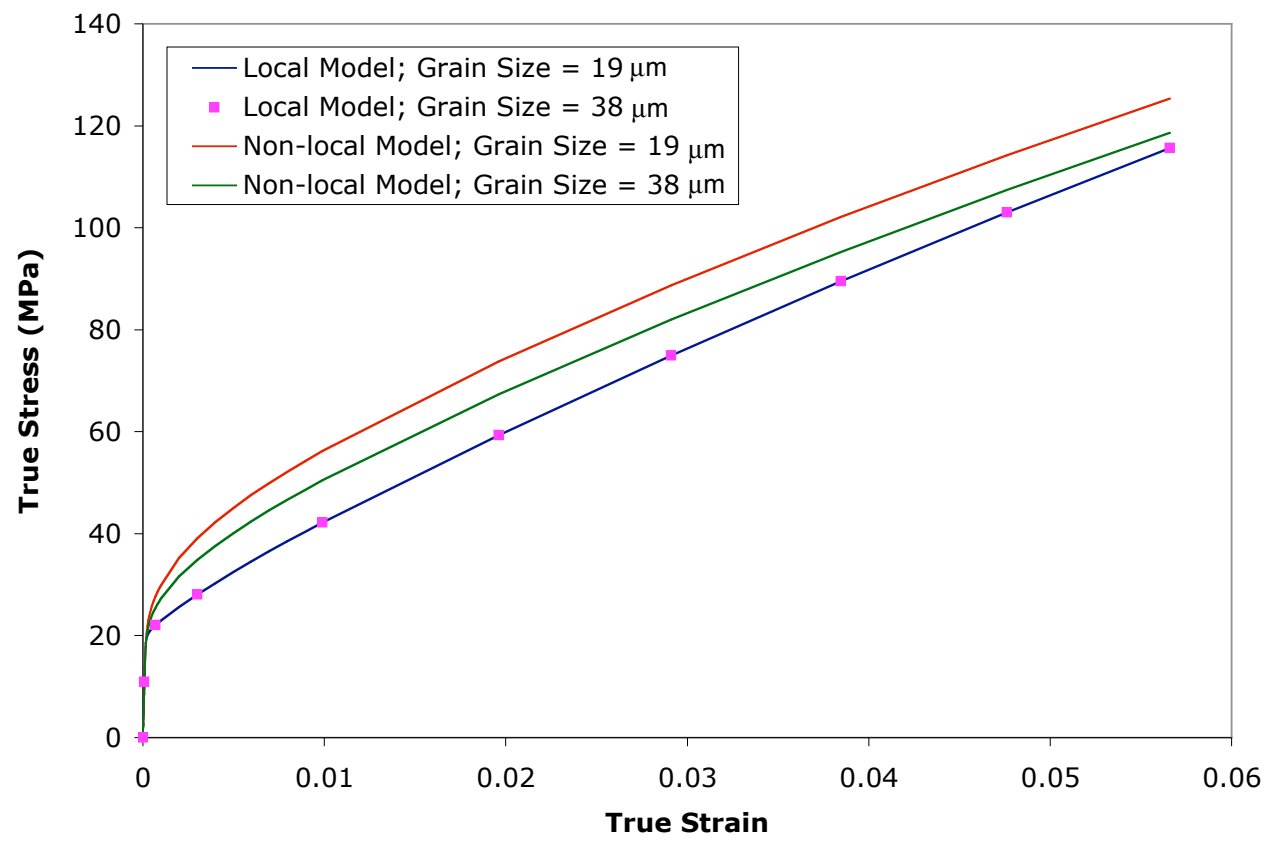

Figure 1.2 - Predicted stress-strain curves from a local and non-local plasticity model for a polycrystal with an average grain size of $19 \mu \mathrm{m}$ and $38 \mu \mathrm{m}$. 
According to the Hall-Petch effect, the simulated stress-strain responses for these two polycrystals should be different. However, the local model predicts the same stress-strain result for the two different grain sizes, while the non-local model does predict a different stress-strain result for the two different grain sizes. The reason for these results is found in the constitutive equations for both models. The constitutive equations governing the stress-strain response in the local model do not have any length parameters (or no internal length scale). Thus the mesh dimensions do not influence the stress-strain equation and the predicted stress strain response of the two grain sizes is similar. The constitutive equations in the non-local model do have length parameters resulting in different stressstrain response for the two polycrystals.

Even though a large number of non-local plasticity models have been proposed since the 1960's, there is no consensus on the best way to formulate such a model. Part of the problem is due to the lack of understanding of how material length scales at the microscale affect the macroscale response. For example, it is generally accepted that geometrically necessary dislocations (GNDs) play a role in many of the aforementioned length scale phenomena. What is the best way to determine the GND state? Do GNDs affect the macroscopic stress-strain response through work hardening or do they create a back stress in the material, or both? Unanswered questions like these have led researchers to propose non-local plasticity models based on very different constitutive equations.

The objective of this research is to develop a non-local crystal plasticity model capable of describing the dependence of yield strength on grain size without the grain 
size explicitly entering into the constitutive equations. The constitutive equations are based on conventional crystal plasticity, but a novel approach based on integrals is used to calculate gradients quantities within the non-local crystal plasticity model. This nonlocal integral approach captures the non-local effects of GNDs (i.e., GNDs at a point harden both the material at that point and surrounding material) and thus predicts a grain size dependence. Because the initial GND state is critical to the material response early in the deformation process (e.g. yielding), a rigorous kinematic analysis is used to determine the initial GND state.

This dissertation is organized as follows. Chapter 2 reviews the experimentally observed length scale dependent phenomena and the prior attempts to model them with continuum plasticity models. Chapter 3 outlines the basics of crystal plasticity theory: kinematics, kinetics, and numerical implementations. Chapter 4 presents the details of the local and non-local crystal plasticity models developed in this research program. Chapter 5 investigates the behavior of the non-local model integral technique used to approximate gradients, in order to better understand the advantages and disadvantages to using this approach. In Chapter 6, the local and non-local crystal plasticity models are applied to polycrystalline materials, and the role of grain boundaries on the Hall-Petch effect is investigated.

Throughout this dissertation, variables written in bold are tensors while variables in regular font are scalars. In index notation, first and second order tensors are denoted by $\mathrm{a}_{\mathrm{i}}$ and $\mathrm{a}_{\mathrm{ij}}$, respectively. The summation over repeated indices is implied, $\sigma_{\mathrm{KK}}=\sigma_{11}+$ $\sigma_{22}+\sigma_{33}$. The inner product of two second-rank tensors is defined as $\mathbf{A} \cdot \mathbf{B}=\mathbf{C}$ where $\mathrm{C}_{\mathrm{ik}}$ $=\mathrm{A}_{\mathrm{ij}} \mathrm{B}_{\mathrm{jk}}$. The scalar product of two second-rank tensors is defined as $\mathbf{A}: \mathbf{B}=\mathrm{A}_{\mathrm{ij}} \mathrm{B}_{\mathrm{ij}}=\mathrm{c}$. 
The dyadic product of two vectors is defined as $\mathbf{a} \otimes \mathbf{b}=\mathrm{a}_{\mathrm{i}} \mathrm{b}_{\mathrm{j}}=\mathrm{C}_{\mathrm{ij}}$. A dot over a variable, $\dot{a}$, implies an ordinary time derivative. Any variable in brackets, $\{a\}$, has been determined using a non-local integral (volume averaging) approach. 


\section{CHAPTER 2}

\section{LITERATURE REVIEW}

In the 1930's, Taylor, Orowan, and Polanyi independently proposed the existence

of line defects, or dislocations, and postulated that crystalline materials plastically deform by breaking atomic bonds along the dislocation lines rather than simultaneously breaking all the atomic bonds on the plane of deformation. Thus, dislocation motion became the primary mechanism for plastic deformation in metallic materials. Other mechanisms of inelastic deformation that complement dislocation motion include diffusive mechanisms that are aided by the mobility of point defects and grain boundary sliding.

Microstructural features (such as other dislocations, precipitates, grain boundaries) can act as barriers that hinder dislocation motion. The increase in stress needed to initiate further plastic deformation is called work hardening. An important consequence of the dislocation concept was that it allows a link to be made between the microstructure and the mechanical response of metallic materials.

Since the 1930's a vast amount of experimental evidence has confirmed the relationship between microstructure and the mechanical response of metallic materials. This relationship has led to the need to define material or microstructure length scales: one-dimensional parameters that quantify the various components that make up the microstructure of a material. Examples of microstructure length scales include particle spacing, grain size, and dislocation mean free path. When the dominant microstructural feature has a length scale less than a few hundred micrometers, the mechanical response of the material changes and the resulting response becomes "length scale dependent". 
Experimentally identifying and measuring these length scales can be difficult and is still an active area of research.

Numerical models have been developed to aid with the interpretation of length scale dependent material behavior and properties. Thus, for a model to correctly capture the physics it must be able to account for microstructure and the resulting microstructural length scales. Local continuum theories cannot predict length scale effects because they have no internal length scale. Atomistic models, on the other hand, can account for length scale dependent behavior. However, these models are computationally limited to impractically short times and material volumes compared to those required for simulations of behavior that is influenced by variables with length scales on the order of microns (Gao et al., 1999a). Thus, non-local continuum theories have been developed. Because these models include an internal length scale term, in theory they are better suited for modeling length scale dependent phenomena. Currently, there is no consensus on the best way to formulate and implement non-local models and thus, it remains an active topic for research. In the subsequent discussion, some of the currently employed models are surveyed with a discussion of their attributes and shortcomings.

\subsection{Dislocation Densities and Microstructure}

\subsubsection{Forest and Glide Dislocations}

The dislocation density in a material is commonly measured using electron microscopy. From a micrograph produced by electron microscopy, it is possible to 
measure the individual dislocation's burgers and tangent vectors and thus determine the direction and plane on which the dislocation can glide conservatively. Thus it is straightforward to classify dislocations based on its slip plane. Dislocations that lie on an active glide plane are called glide dislocations and make up the glide dislocation density $\left(\rho^{\mathrm{G}}\right)$. Dislocations that intersect or pass through an active glide plane are called forest dislocations and make up the forest dislocation density $\left(\rho^{\mathrm{F}}\right)$. An example of glide and forest dislocations is shown in Figure 2.1.

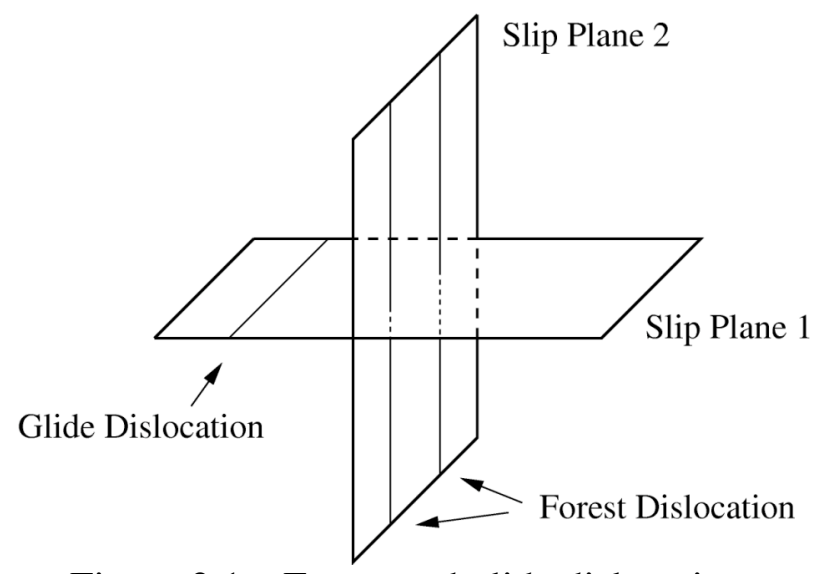

Figure 2.1 - Forest and glide dislocations

The active slip plane in Figure 2.1 is slip plane 1 making the dislocation in this plane a glide dislocation. Dislocations on slip plane 2 that intersect slip plane 1 are forest dislocations.

Even in well-annealed specimens, the number of dislocations is sufficiently high to ensure that dislocations will thread every plane within the material. So an appreciable number of forest dislocations are always present in any material. A glide dislocation moving on its slip plane within a crystal is bound to interact with forest dislocations. 
These forest dislocations can impede the motion of glide dislocations via a number of different dislocation-dislocation interactions (for example: jog/kink formation and stress field interactions). Therefore $\rho^{\mathrm{F}}$ represents an identifiable length scale parameter that influences the deformation behavior.

\subsubsection{Geometrically Necessary and Statistically Stored Dislocations}

As described above, the forest and glide dislocations separates dislocations based on whether a dislocation lies on an active slip plane or it intersects an active slip plane. Dislocations can also be divided based on their role within the material. In certain situations, dislocations are needed to accommodate the deformation field. One such example is bending, shown in Figure 2.2.

a)

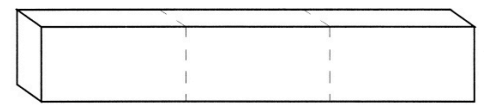

b)

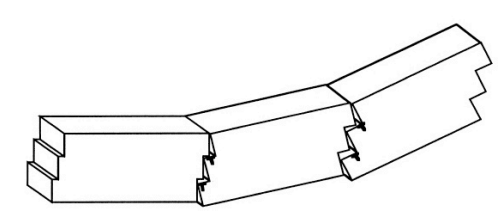

c)

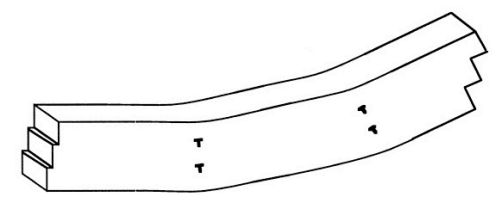

Figure 2.2 - Dislocations needed to accommodate a bending curvature (Arsenlis and Parks, 1999)

The dislocations in Figure 2.2 that are required by the deformation field are called geometrically necessary dislocations (GNDs). All other dislocations within the material are called statistically stored dislocations (SSDs).

Nye (1953) developed the concept that Ashby (1970) would later call GNDs. Nye 
(1953) defined GNDs as the dislocations that give rise to a net burgers vector, or closure failure of a burgers circuit. All dislocations that do not contribute to the net burgers vector, which in effect is all other dislocations, were defined as SSDs. An example of a burgers circuit enclosing both GNDs and SSDs is also shown in Figure 2.3.

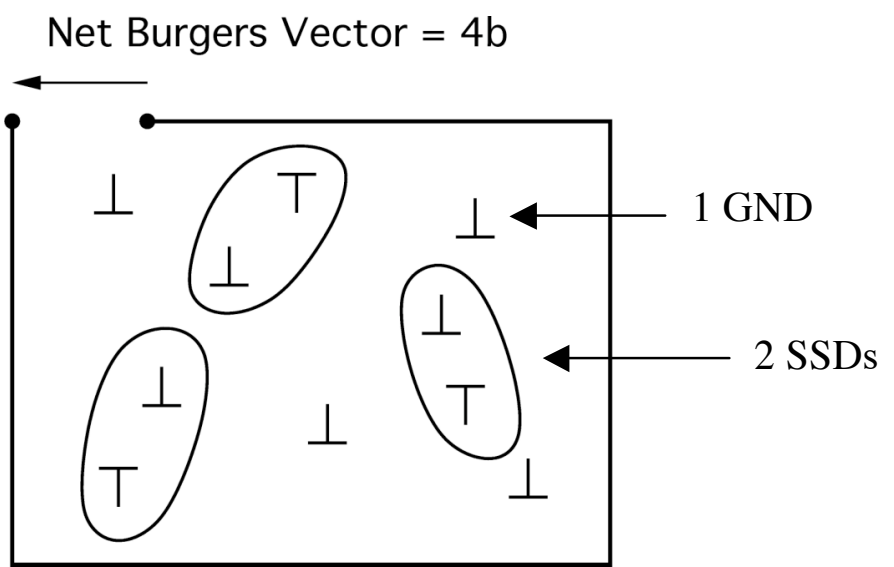

Figure 2.3 - A burgers circuit around GNDs and SSDs

The net burgers vector of $4 \mathrm{~b}$ in Figure 2.3 is due to the four unpaired dislocations or GNDs enclosed by the burgers circuit. Each dislocation pair containing dislocations with opposite signs contributes nothing to the net burgers vector because the individual burgers vectors within the pair cancel out. Thus both dislocations within the pair are SSDs.

Figure 2.3 illustrates the subjectivity of the GND/SSD concept: the dislocations can be regrouped in a number of different ways that will result in the same net burgers vector. This also underscores an important point that fundamentally GNDs and SSDs are not different in characteristics but are distinct only in the role they play in accommodating the deformation. In addition, the GND content depends on the size of the burgers circuit. If a burgers circuit was on the order of dislocation spacing, then 
every dislocation in Figure 2.3 would be a GND. If, on the other hand, the burgers circuit was very large (much larger than that shown in Figure 2.3), then the GND content would go to zero.

Ashby (1970) applied the concept of GNDs to the work hardening response of materials that deform in a non-uniform manner. Non-uniform deformations arise in a material as a result of the non-uniformity in the stress distribution or in the material's microstructure. Some examples of non-uniform deformations requiring the presence of GNDs are shown in Figure 2.4.

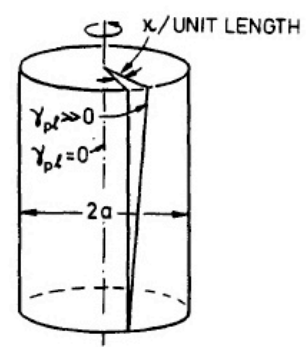

(a)

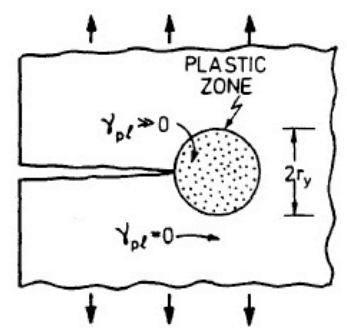

(d)

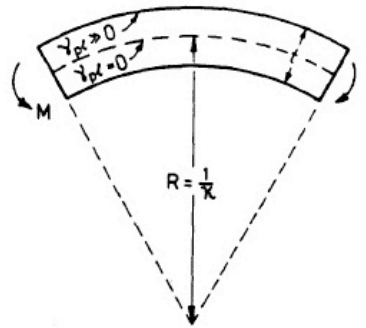

(b)

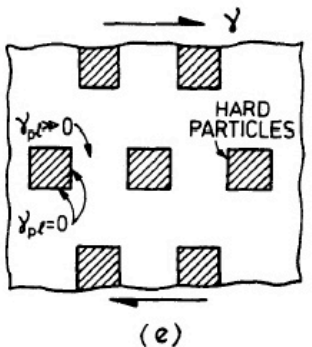

(e)

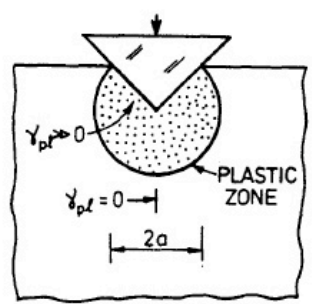

(c)

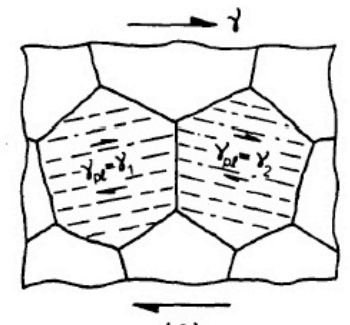

(f)

Figure 2.4 - Non-uniform deformations resulting from the applied load (a,b,c,d) or the material's microstructure (e,f). (Fleck et al., 1994)

In Figure 2.4, dislocations are geometrically necessary to ensure compatibility during deformation. Thus, the number of GNDs is related in some way to the non-uniform 
deformation.

Ashby (1970) considered a number of different non-uniform deformation scenarios, but his treatment of polycrystalline materials is of particular interest to this discussion. Ashby (1970) noted that the different orientation of each grain causes a different stress response in each grain. Without the constraint of neighboring grains, the different stress response of each grain would cause overlaps and voids in the polycrystal. Ashby (1970) postulated that GNDs are needed in polycrystalline materials to preserve compatibility between neighboring grains as illustrated in Figure 2.5.
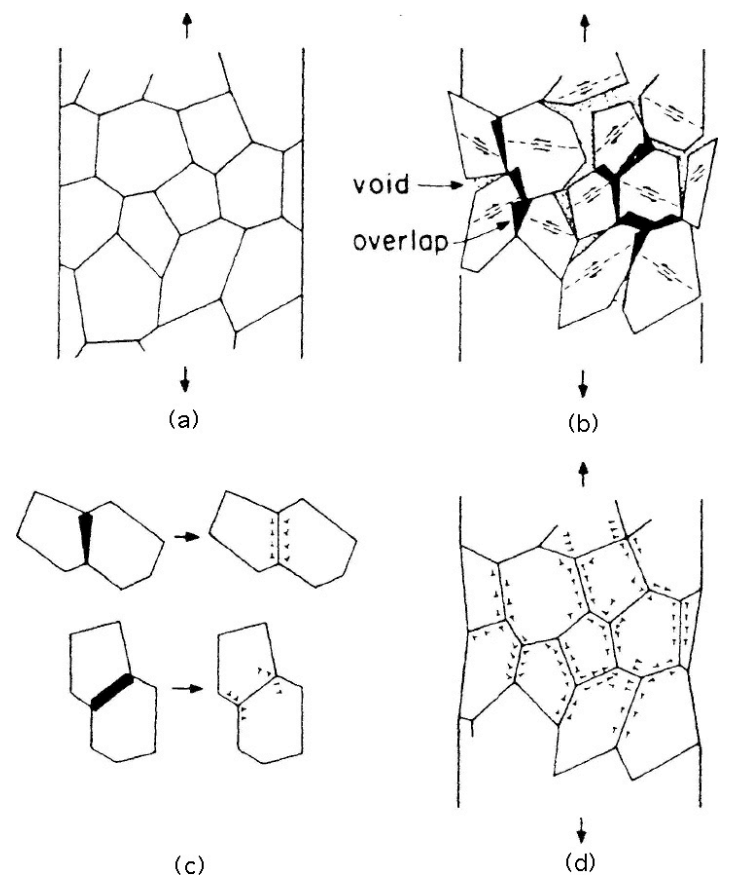

Figure 2.5 - If each grain in the polycrystal shown at (a) deforms without the constraint of its neighbors, voids and overlaps appear as seen in (b). These voids and overlaps can be eliminated by GNDs as shown in (c) and (d). (Ashby, 1970)

Ashby (1970) also provided some physical insight into SSDs. He suggested that SSDs accumulate due to random dislocation-dislocation interactions within the crystal. 
Direct transmission electron microscope measurements (TEM) of GND densities $\left(\rho^{\mathrm{GND}}\right)$ are impossible. A TEM micrograph does not reveal any information about a net burgers vector or a non-uniform deformation state. The density of SSDs $\left(\rho^{\text {SSD }}\right)$ can be measured in single crystals deformed in uniform tension since the resulting deformation field is completely uniform leading to a dislocation density made up entirely of SSDs. Ashby (1970) proposed that $\rho^{\mathrm{GND}}$ could be characterized by a "geometric slip distance" $\left(\lambda^{\mathrm{GND}}\right)$ via

$$
\rho^{\mathrm{GND}} \approx \frac{4 \gamma}{\lambda^{\mathrm{GND}} \mathrm{b}}
$$

where $\gamma$ is the macroscopic plastic shear strain and $b$ is the magnitude of the burgers vector. Figure 2.6 shows the comparison of $\rho^{\mathrm{SSD}}$ from single crystals with $\rho^{\mathrm{GND}}$ estimated from Eq. (2.1) with various values of $\lambda^{\mathrm{GND}}$.

At $10 \%$ strain, $\rho^{\mathrm{GND}}>>\rho^{\mathrm{SSD}}$ for $\lambda^{\mathrm{GND}}<40 \mu \mathrm{m}$ for single crystals and $\lambda^{\mathrm{GND}}<10 \mu \mathrm{m}$ for polycrystals. $\rho^{\mathrm{SSD}}>>\rho^{\mathrm{GND}}$ at $10 \%$ strain for $\lambda^{\mathrm{GND}}>100 \mu \mathrm{m}$ for single crystals and $\lambda^{\mathrm{GND}}>20 \mu \mathrm{m}$ for polycrystals. Thus, there are regimes where either $\rho^{\mathrm{SSD}}$ or $\rho^{\mathrm{GND}}$ are the predominant dislocation type within the material. There is a microstructural length scale associated with both GNDs $\left(\lambda^{\mathrm{GND}}\right)$ and SSDs $\left(\lambda^{\mathrm{SSD}}\right)$. The length scale that dominates the material's response is the length scale associated with the dominant microstrucutral feature. 


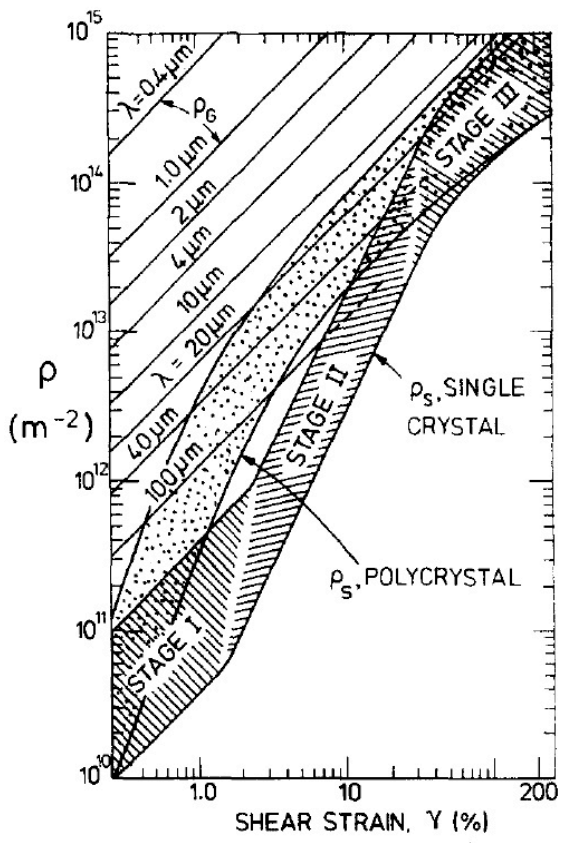

Figure 2.6 - Comparison of $\rho^{\mathrm{GND}}$ and $\rho^{\mathrm{SSD}}$. Single crystal data was taken from Basinski and Basinski (1966). Fleck et al. (1994) inferred the polycrystal data from stress-strain curves. In this figure $\lambda=\lambda^{\mathrm{GND}}$.

At $10 \%$ strain, $\rho^{\mathrm{GND}}>>\rho^{\mathrm{SSD}}$ for $\lambda^{\mathrm{GND}}<40 \mu \mathrm{m}$ for single crystals and $\lambda^{\mathrm{GND}}<10 \mu \mathrm{m}$ for polycrystals. $\rho^{\mathrm{SSD}}>>\rho^{\mathrm{GND}}$ at $10 \%$ strain for $\lambda^{\mathrm{GND}}>100 \mu \mathrm{m}$ for single crystals and $\lambda^{\mathrm{GND}}>20 \mu \mathrm{m}$ for polycrystals. Thus, there are regimes where either $\rho^{\mathrm{SSD}}$ or $\rho^{\mathrm{GND}}$ are the predominant dislocation type within the material. There is a microstructural length scale associated with both GNDs $\left(\lambda^{\mathrm{GND}}\right)$ and SSDs $\left(\lambda^{\mathrm{SSD}}\right)$. The length scale that dominates the material's response is the length scale associated with the dominant microstrucutral feature.

\subsection{General Dislocation Density-Property Relationships}

Taylor (1934) proposed that a metallic material can work harden as a result of 
dislocation-dislocation interactions. He considered two edge dislocations on parallel slip planes a distance $\mathrm{L}$ apart. The stress required to force one edge dislocation past the other was termed the critical shear stress $\left(\tau^{\mathrm{CSS}}\right)$ and mathematically defined as

$$
\tau^{\mathrm{CSS}}=\frac{\mathrm{CGb}}{\mathrm{L}}
$$

where $\mathrm{C}$ is a material constant on the order of 1 and $\mathrm{G}$ is the shear modulus. Considering a regular array of edge dislocations with a spacing $\mathrm{L}$, the average total dislocation density, $\langle\rho\rangle$, of these dislocations is $\langle\rho\rangle \cong \mathrm{L}^{-2}$. Equation (2.2) was then written as

$$
\tau^{\mathrm{CSS}}=\mathrm{CGb} \sqrt{\langle\rho\rangle} \text {. }
$$

Equation (2.3) is the well-known Taylor equation.

Verification of the Taylor equation has come in two forms. The first starts with the assumption that $\gamma$ is proportional to dislocation density via

$$
\gamma \propto\langle\rho\rangle \mathrm{b} \overline{\mathrm{l}}
$$

where $\bar{i}$ is the average dislocation mean free path. Substituting Eq. (2.4) into Eq. (2.3) results in a stress-strain equation that predicts a parabolic stress-strain curve, characteristic of many polycrystalline materials. The other corroboration of Taylor's equation is based on the experimental measurement of forest dislocation densities vs. flow stress. Mecking and Kocks (1981) compared experimentally measured flow stresses and flow stresses predicted by Taylor's equation for the same measured $\rho^{\mathrm{F}}$. Their comparison for pure copper deformed at room temperature is shown in Figure 2.7. 


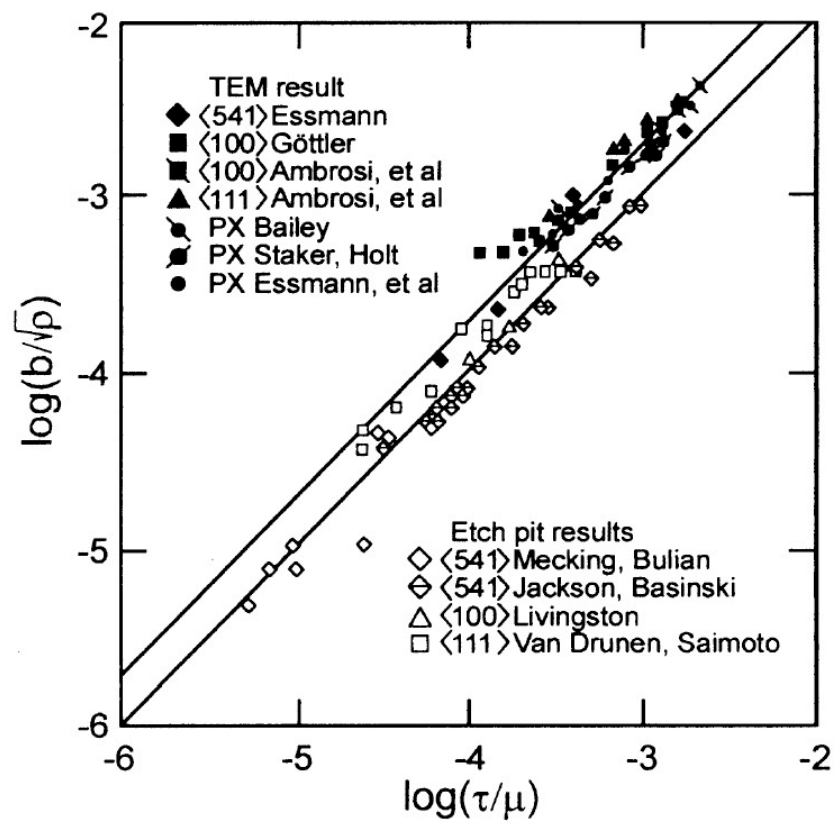

Figure 2.7 - Dislocation density vs. flow stress. The straight lines in Figure 7 represent $\tau=\mathrm{CGb} \sqrt{\rho^{\mathrm{F}}}$ with $\mathrm{C}=1$ and 0.5. (Mecking and Kocks, 1981)

From this data, Mecking and Kocks (1981) concluded, "the proportionality between the flow stress (due to dislocation/dislocation interactions) and the square-root of the (forest) dislocation density is well substantiated."

The influence of GNDs and SSDs on the mechanical response of a material has been incorporated into the Taylor equation, Eq. (2.3), by making the average total dislocation density a function of $\rho^{\mathrm{GND}}$ and $\rho^{\mathrm{SSD}}$. Assuming that $\rho^{\mathrm{GND}}$ and $\rho^{\mathrm{SSD}}$ do not interact with each other, $\langle\rho\rangle$ has been expressed as

$$
\langle\rho\rangle=\rho^{\mathrm{GND}}+\rho^{\mathrm{SSD}} .
$$

Ashby (1970) pointed out that the presence of $\rho^{\mathrm{GND}}$ will influence $\rho^{\mathrm{SSD}}$, and therefore Eq. (2.5) represented a lower bound for the total effective dislocation density. Fleck and Hutchinson (1997) proposed that the $\rho^{\mathrm{GND}}$ and $\rho^{\mathrm{SSD}}$ interact such that the $\langle\rho\rangle$ could be 
written as a harmonic sum

$$
\langle\rho\rangle^{\mathrm{u}}=\left(\rho^{\mathrm{GND}}\right)^{\mathrm{u}}+\left(\rho^{\mathrm{SSD}}\right)^{\mathrm{u}} .
$$

Duan et al. (2001) used Eq. (2.6) with a u less than 1 to obtain a higher $\langle\rho\rangle$ than that predicted by Eq. (2.5). Finally, Mughrabi (2001) suggested that GNDs on one slip system can act as forest dislocations for another slip system. Thus $\langle\rho\rangle$ became

$$
\langle\rho\rangle=\rho^{\mathrm{SSD}}+\mathrm{c}_{\mathrm{MU} 1} \rho^{\mathrm{GND}}
$$

where $\mathrm{C}_{\mathrm{MU}}$ takes into account the geometry of the interaction between glide and forest dislocations.

\subsection{Experimental Observations of Length Scale Dependent Phenomena}

\subsubsection{Grain Size Effects}

The Hall-Petch relationship was originally proposed by Hall (1951) and Petch (1953) and based on their work with mild steels. They observed that the yield strength $\left(\sigma_{\mathrm{y}}\right)$ of polycrystalline mild steel depends on the grain size $(\mathrm{d})$ via

$$
\sigma_{\mathrm{y}}=\sigma_{0}+\mathrm{kd}^{-\frac{1}{2}}
$$

where $\sigma_{0}$ is the initial yield strength, and $\mathrm{k}$ is a material parameter known as the HallPetch slope. Since then, this relationship has been observed in a number of other materials: 70:30 brass (Meakin and Petch, 1974), copper (Feltham and Meakin, 1957), aluminum (Carreker and Hibbard, 1957), nickel (Thompson, 1975), zinc (Armstrong et al., 1962), and silver (Carreker, 1957), to name a few. A typical Hall-Petch response is illustrated in Figure 2.8. 


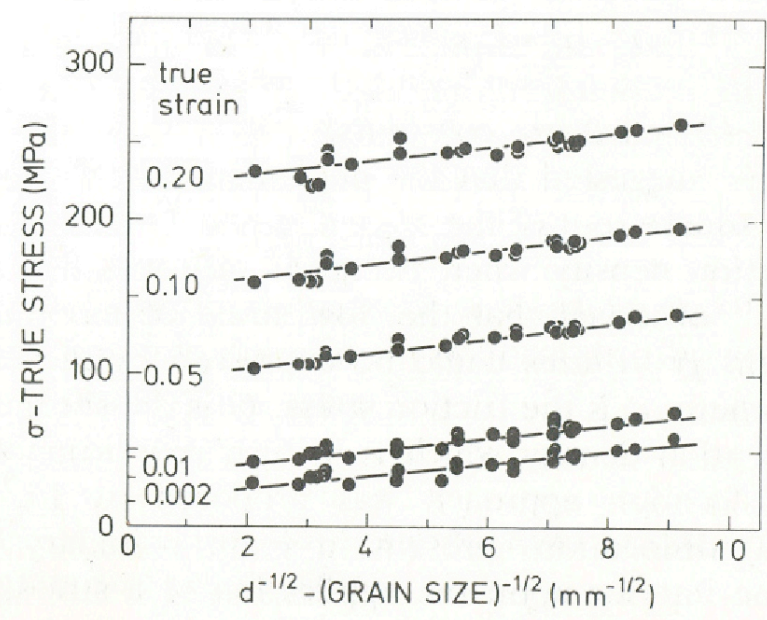

Figure 2.8 - Hall-Petch response of copper for at a number of different strains. (Hansen, 1983)

There has been some evidence for a Hall-Petch exponent other than $-1 / 2$ leading to a generalized Hall-Petch equation

$$
\sigma_{\mathrm{y}}=\sigma_{0}+\mathrm{kd}^{-\mathrm{n}}
$$

where $\mathrm{n}$ is the Hall-Petch exponent. Baldwin (1958) noted that there is enough scatter in the data to support either an exponent of -1 or $-1 / 3$. However, the bulk of the experiments support an exponent of $-1 / 2$.

Hall (1951) and Petch (1953) proposed a dislocation pile-up model to account for the experimentally observed grain size dependence. They suggested that dislocations of like sign generated from a Frank-Reed source piled-up at grain boundaries because grain boundaries act as barriers to dislocation motion. Yielding, or flow, takes place once the stress at the head of the pile-up exerted enough stress to propagate plastic deformation from one grain to another. Cottrell (1958) used the pile-up model and assumed yielding 
occurred once the stress at the head of the pile-up was sufficient to activate Frank-Reed sources near the grain boundary in the adjacent grain.

Analytic dislocation calculations using the pile-up model do support Eq. (2.8); however, the lack of experimentally observed pile-ups in pure metals led Li (1963) to propose a different mechanism to explain the grain size effect. Li (1963) suggested that grain boundary ledges could act as dislocation sources without the stress generated by a pile-up. These grain boundary ledges become lattice dislocations when they are released to the interior of the grain creating a dislocation forest in the interior of the grain.

Yielding occurs when there is enough stress to move dislocations through the dislocation forest created by grain boundary ledges. Electron microscopic observations of grain boundary ledges confirm their existence and atomistic calculations confirm that grain boundary ledges can act as dislocation sources.

Other non pile-up mechanisms proposed to explain the Hall-Petch effect are based on the existence of compatibility stresses in the grain boundary region. Hook and Hirth (1967) studied slip operation in the grain boundary region of bicrystals oriented for easy glide. In some of the grain boundary regions, secondary slip was observed in the absence of primary slip. They suggested that this secondary slip was caused by elastic incompatibility stresses resulting in anisotropic response of each grain to the applied load. Hook and Hirth (1968) also observed a diminishing intensity of screw dislocation slip bands near the bicrystal grain boundary. They attributed this effect to a repulsive image stress at the boundary. Hirth (1972) states that these stresses can produce two hardening effects in the grain boundary region: (1) Repelling dislocations from the boundary and thereby increasing local internal stress resisting dislocation motion, and (2) 
Promoting cross-slip, which can create a dislocation structure that increases the local flow stress of the region.

\subsubsection{Indentation}

Indentation has been used for many years to test the material properties of small volumes near the surface. If these indentation experiments were length scale independent, the measured properties like hardness would be independent of the indenter size and depth of the indent. However, Gane and Cox (1970) reported an increase in hardness down to depths of $0.2 \mu \mathrm{m}$ in both work hardened and annealed gold samples. In addition, they reported a dependence of hardness on the radius of the spherical indenter tip. Pethica et al. (1983) later verified the length scale dependence of hardness, which was later called the Indentation Size Effect (ISE). They observed that hardness increased as indent size decreased to depths as low as $20 \mathrm{~nm}$. Figure 2.9 shows a typical ISE result.

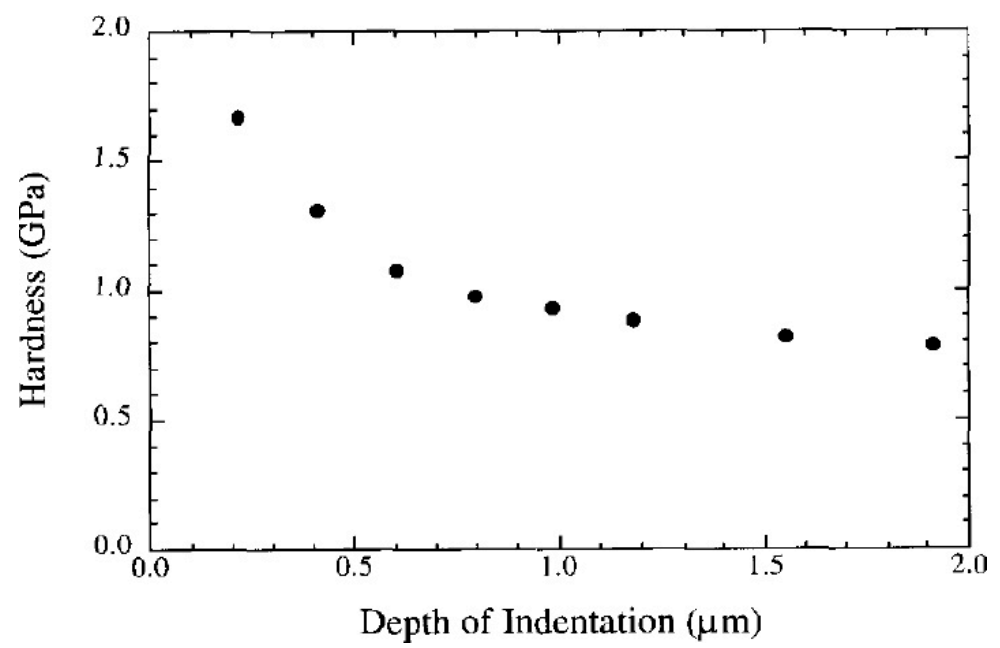

Figure 2.9 - The ISE in copper. (McElhaney et al., 1998) 
The development of a commercially available sub-micron indenter with extremely fine load-displacement control and the creation of standard procedures to determine the contact area, hardness, and moduli from load-displacement data allowed sub-micron indentation to become available to a large number of researchers in the 1990s. Thus, a number of sub-micron indentation studies were conducted on different materials, with different orientations, and different initial microstructures to verify the ISE under different conditions. Oliver and Pharr (1991) tested a number of metallic and nonmetallic materials. They reported no size effect on hardness for the 4 non-metallic materials they tested (sapphire, quartz, fused silica, and soda-lime glass). However, they did observe a modest increase in hardness for low loads at lower depths in the 2 metallic materials tested (single crystal aluminum and tungsten). Nix (1997) later pointed out that the ISE is limited to materials that strain harden. Stelmashenko et al. (1993) investigated the effect of different surface orientations on the hardness of tungsten and molybdenum single crystals. They observed an increase in hardness below loads of $100 \mathrm{mN}$ on each of the surfaces with the (100) surface being the hardest and the (111) surface the softest. Finally, Poole et al. (1996) indented both work hardened and annealed copper. They observed the ISE in both the work-hardened and annealed specimens. In addition, the hardness of the work-hardened copper was higher than that of the annealed copper.

On the whole, there is ample experimental evidence that the hardness of metallic materials does depend on depth. However, the mechanisms at the microstructural level that cause the ISE are still not well understood. Early on, Gane and Cox (1970) and Pethica et al. (1983) postulated that the increase in hardening was in some way related to an increase in the stress necessary to operate dislocation sources. Currently, it is widely 
believed that that the ISE is caused by GNDs. Stelmashenko et al. (1983) were one of the first to link the ISE with GNDs. They observed that the plastic displacement field caused by indentation could be compressive or rotational as shown in Figure 2.10.

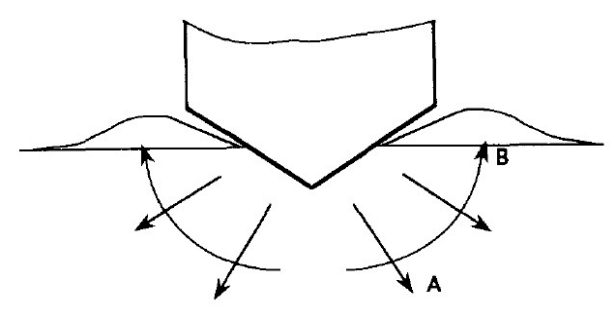

Figure 2.10 - Compressive (A) and Rotational (B) displacement fields under an indenter tip. The rotational plastic displacement field results in GND storage. (Stelmashenko et $a l ., 1983)$

Each increment of plastic flow resulting from the indenter pushing farther in the material is accompanied by a decrease in the GND density (not necessarily a decrease in the number of GNDs) since they are spread over a larger volume. Based on the Taylor's equation relating flow stress and dislocation density, Stelmashenko et al. (1983) derived a relationship that predicted a linear relation between hardness $(\mathrm{H})$ squared and the reciprocal of indentation depth $(\varpi)$ as

$$
\mathrm{H}=\mathrm{ACGb}\left(\rho^{\mathrm{SSD}}+\frac{\cot \Gamma}{\mathrm{b} \Phi}\right)^{\frac{1}{2}}
$$

where, $\mathrm{A}$ is the ratio between normal stress and hardness and $\Gamma$ is the angle between opposite faces of indent. This approach was also used by Ma and Clarke (1995), Poole et al. (1996), and Nix and Gao (1998) with different expressions for $\rho_{\mathrm{GND}}$ to derive a linear relationship between hardness squared and indentation depth. Poole et al. (1996) 
observed a linear relationship between $\mathrm{H}^{2}$ and $\varpi^{-1}$ in both the work-hardened and annealed copper. Nix and Gao (1998) observed excellent agreement between their hardness model and the indentation data of McElhaney et al. (1998) and Ma and Clarke (1995), further supporting the suggestion that there is a linear relation between $\mathrm{H}^{2}$ and $\sigma^{-1}$

\subsubsection{Specimen Dimension Effects in Bending and Torsion}

Experiments also indicate the existence of a non-microstructure related length scale associated with specimen dimensions. Fleck et al. (1994) observed an increase in the work hardening in torsional response of polycrystalline copper wires as the diameter of the wire decreased from $170 \mu \mathrm{m}$ to $12 \mu \mathrm{m}$. They also observed a greater influence of specimen diameter in torsion compared with tension, as shown in Figure 2.11. Stolken and Evans (1998) reported a similar observation for the bending response of polycrystalline nickel foils. Using a microbend test method that relied on measuring the curvature of the bent specimen rather than the bending moment, they observed an increase in the work hardening of polycrystalline nickel foils with thicknesses between 50um and $12.5 \mathrm{um}$. Haque and Saif (2003) developed a cantilever bending procedure to study the mechanical response of polycrystalline aluminum foils. They also observed an increase in the rate of work-hardening as the foil thickness decreased from $485 \mathrm{~nm}$ to $100 \mathrm{~nm}$. 


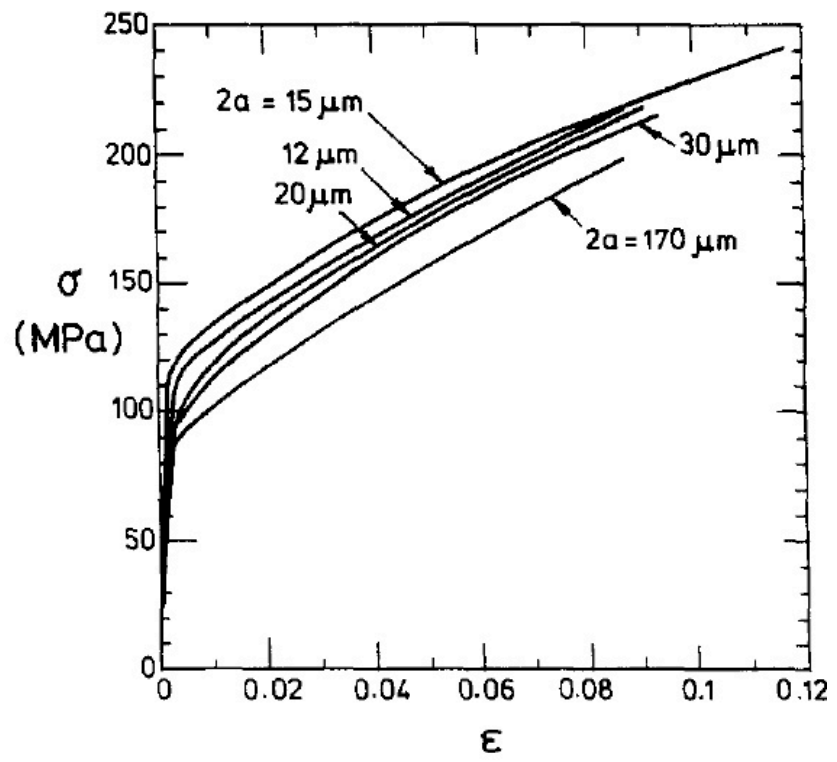

(a) Tension

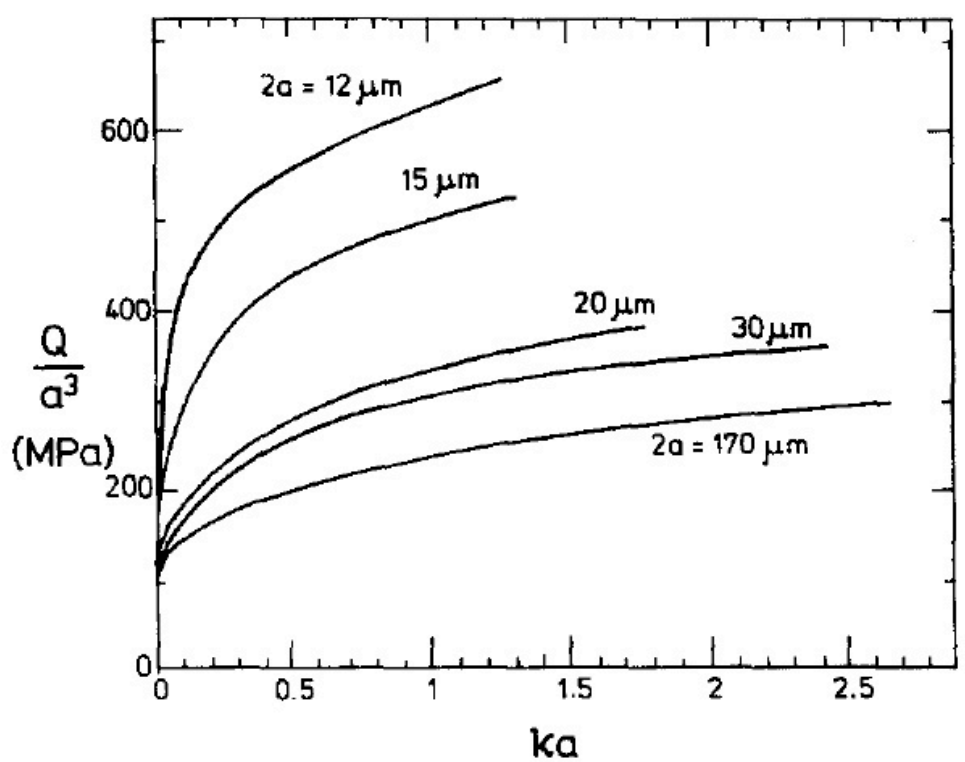

(b) Torsion

Figure 2.11 - Tension and torsion response of copper wires with diameters of $12 \mu \mathrm{m}$ to $170 \mu \mathrm{m}$. (Fleck et al., 1994) 


\subsubsection{Specimen Dimension Effects in Tension and Compression}

Length scale effects associated with specimen dimension have been observed under tensile and compressive loads. These length scale effects have been observed in both free-standing thin films (thin films without a substrate) and traditional cylinder shaped specimens.

\subsubsection{Free Standing Films}

Huang and Spaepen (2000) tested the tensile properties of a multilayered $\mathrm{Ag} / \mathrm{Cu}$ free standing thin films. This multilayered material consisted of $\mathrm{Ag}$ and $\mathrm{Cu}$ layers of equal thickness ranging from $1.5 \mathrm{~nm}$ to $1.5 \mu \mathrm{m}$, but the overall thickness of the multilayered material was kept constant at $3 \mu \mathrm{m}$. Huang and Spaepen (2000) observed an increase in the yield strength as the layer thickness got smaller and the data nicely fit the Hall-Petch equation, $\mathrm{n}=-0.5, \sigma_{0}=223 \mathrm{MPa}$, and $\mathrm{k}=0.104 \mathrm{MPa} \sqrt{\mathrm{m}}$.

Espinosa et al. (2004) investigated the properties of polycrystalline $\mathrm{Au}, \mathrm{Cu}$, and Al free-standing films using the membrane deflection experiment, which achieves "direct tensile stressing of the specimens". The width of the films tested ranged from $2.5 \mu \mathrm{m}$ to $20 \mu \mathrm{m}$ and the thickness between $0.2 \mu \mathrm{m}$ and $1 \mu \mathrm{m}$. The difference in the stress-strain response for a $1.0 \mu \mathrm{m}$ and $0.2 \mu \mathrm{m}$ free standing film is shown in Figure 2.12. 


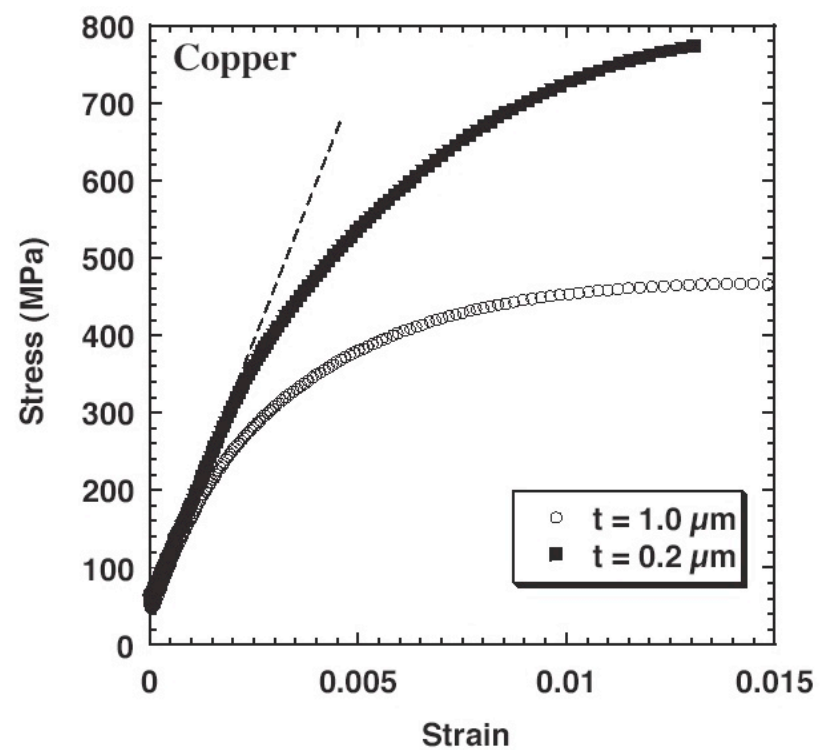

Figure 2.12 - Stress-strain response of a 1.0 and $0.2 \mu \mathrm{m}$ free-standing film (Espinosa $e t$ al., 2004)

The elastic response of these films was unaffected by specimen dimensions. However, the yield strength (defined as the stress at which the $\sigma$ - $\varepsilon$ curve deviates from linearity) and the rate of work hardening increased as either/both the specimen dimensions were reduced. Analysis of the thin films showed that the number of grains along the thickness and width decreased as the specimen dimension became smaller. This observation led Espinosa et al. (2004) to conclude that the increase in the yield strength due to a reduction in specimen dimensions resulted from geometric constraints associated with the small number of grains and the reduction in the number of dislocation sources.

\subsubsection{Cylindrical Specimens}

Length scale effects have also been observed in single crystals loaded in tension and compression. In these experiments, the stress state in the material is uniform 
resulting in a material free of strain gradients. Therefore the microstructural mechanisms for the observed length scale effect cannot be solely due GNDs.

It is well known that the mechanical response of single crystal metal whiskers is very different than that of bulk metallic crystals. Suzuki et al. (1956) tested single crystal copper specimens with radii between $60 \mu \mathrm{m}$ and $1 \mathrm{~mm}$. In general, Suzuki et al. (1956) observed the classic single crystal stress-strain curves. They reported a strong effect of radii on the easy glide (Stage I) response, but a weak affect of radii on work hardening (Stage II). Brenner (1956) tested $\mathrm{Fe}, \mathrm{Cu}$, and Ag single crystal whiskers with diameter between $1.2 \mu \mathrm{m}$ and $15 \mu \mathrm{m}$. Unlike Suzuki et al. (1956), Brenner (1956) did not observe a Stage I/Stage II type of stress-strain curve. Rather, the stress-strain curves were primarily linear with a small amount of curvature prior to fracture. Brenner (1956) also reported that the average strength (maximum stress prior to fracture) was inversely proportional to the specimen diameter: the smaller the diameter, the higher the strength. The increase in the ultimate tensile for the smallest whiskers was 10-50 times that of bulk crystals. The large amount of scatter in the strength data led Brenner (1956) to postulate that the strength of a perfect whisker is decreased by surface and/or internal defects, which are "distributed statistically". A smaller whisker has a smaller chance of having a defect and therefore a higher the average strength.

Recently, Uchic et al. (2004) used a focused ion beam (FIB) to machine cylindrical compression specimens with diameters between $0.5 \mu \mathrm{m}$ and $40 \mu \mathrm{m}$ into the surface of a bulk crystal. These specimens were then compressed using a nanoindentor with a flat punch. The use of a nanoindentor as a load frame made accurate loaddisplacement measurements possible. The stress strain curves for Ni specimens with a 
diameter between $20 \mu \mathrm{m}$ and $40 \mu \mathrm{m}$ oriented for single slip were similar to those of bulk samples. Specifically, the yield strength and work hardening rates were within $30 \%$ of those for millimeter sized specimens. The stress-strain curves for all these specimens had a distinct elastic region followed by plastic deformation characterized by short periods of stable flow with low work hardening rates, separated by increments of elastic loading as shown in Figure 2.13.

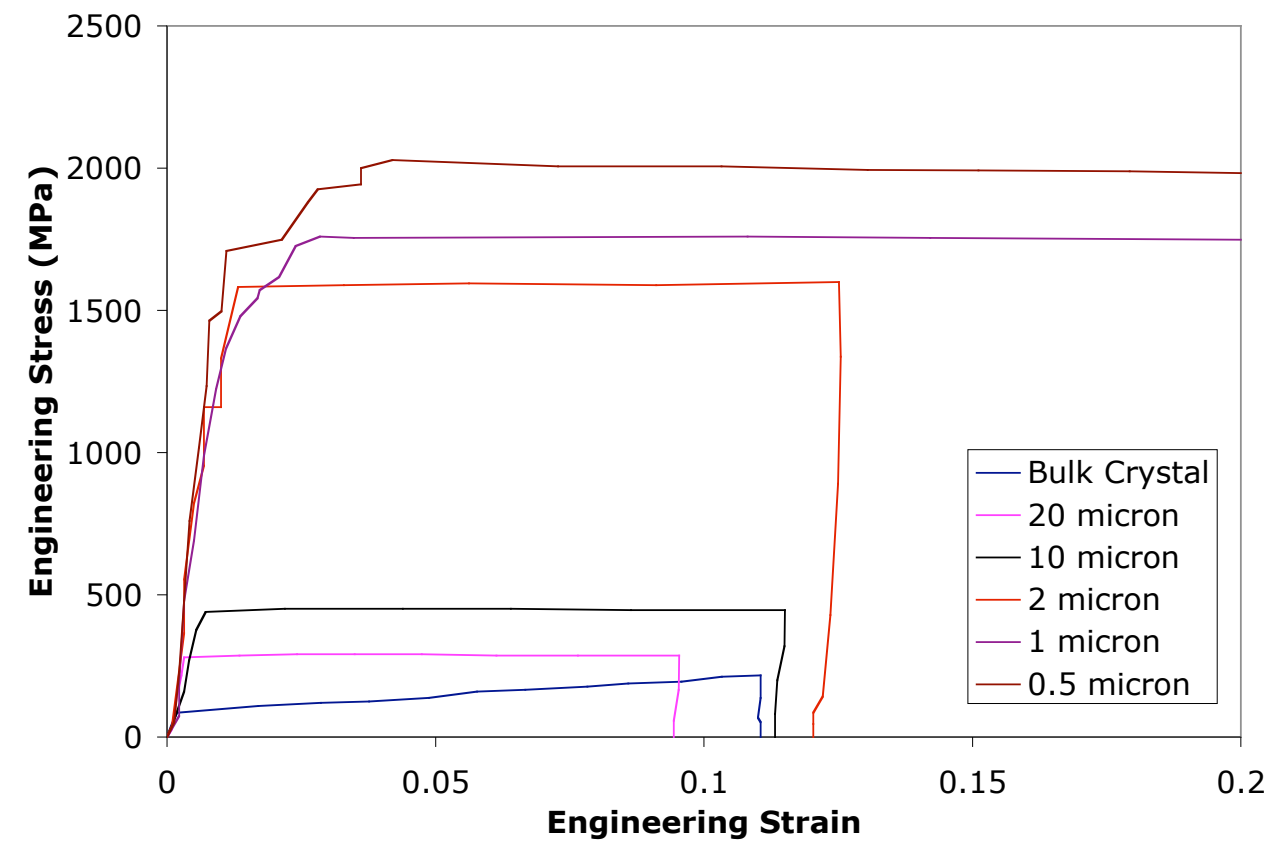

Figure 2.13 - Stress-strain curves for the micron sized compression specimens (Uchic et al., 2004)

Changes in the stress-strain response were observed for specimens with a $5 \mu \mathrm{m}$ diameter.

These samples do not demonstrate a smooth transition between elastic and plastic behavior. Rather yielding appears to be the result of large strain bursts: rapid plastic flow to values of up to $19 \%$ strain with no work hardening. In addition, an increase in yield 
strength was observed for each of the four $5 \mu \mathrm{m}$ diameter specimens compared to the $20 \mu \mathrm{m}$ specimens.

Greer et al. (2005) used the experimental approach developed by Uchic et al. (2004) to investigate the mechanical response of small free-standing gold pillars with diameters ranging from $0.3 \mu \mathrm{m}$ to $7.45 \mu \mathrm{m}$. Qualitatively, the results of Greer et al. (2005) were similar to those of Uchic et al. (2004). The Au stress-strain curves exhibited periods of stable elastic loading followed by strain bursts. The strain bursts in Au were not as large as those observed in Ni. Greer et al. (2005) also observed a gradual increase in the flow stress as the pillar diameter decreased from $7 \mu \mathrm{m}$ to $1 \mu \mathrm{m}$, at which point the flow stress rapidly increased as the diameter was decreased further to $0.3 \mu \mathrm{m}$, as shown in Figure 2.14.

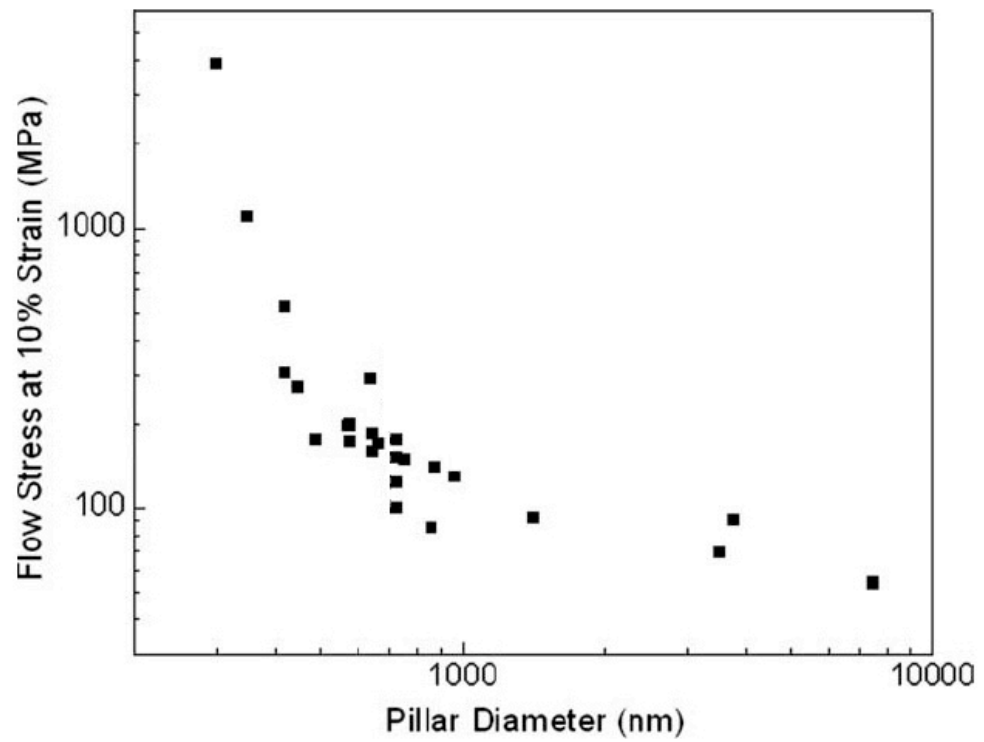

Figure 2.14 - Effect of pillar diameter on flow stress (Greer et al., 2005) 
The flow stress at 10\% strain reached as high as $4.5 \mathrm{GPa}$. Greer et al. (2005) offer a possible explanation for these high strengths in uniaxial compression based on dislocation starvation. In specimens with small physical dimensions, the distance a dislocation must travel before annihilation at a free surface is small. This small mean free path leads to a reduction in the probability that the dislocation will participate in dislocation multiplication processes. If all the dislocations are swept out and do not multiply, very high stress would be needed to nucleate new dislocations leading to the observed high flow stresses.

\subsection{Numerical Modeling of Length Scale Phenomena}

\subsubsection{Modeling Length Scale Phenomena with Local Models}

Classical solid mechanics continuum models have been built on the assumption that the state of a point in the body depends on only the current values and possibly the history of deformation and temperature at that point only. Models based on this assumption are classified as local models. Local models have been used to simulate length scale dependent phenomena, specifically the Hall-Petch effect.

The experimental evidence suggesting the grain boundary region deforms differently than the grain interior leads naturally to modeling a polycrystal as a composite consisting of two phases: (1) A hard grain boundary region of constant width with a flow stress $\sigma_{\mathrm{GB}}$ and (2) A grain interior region with a flow stress $\sigma_{\mathrm{I}}$. Kocks (1970) proposed that the yield strength of the polycrystal could be obtained by a rule-of-mixtures based 
averaging procedure

$$
\sigma_{\mathrm{y}}=\sigma_{\mathrm{I}} \mathrm{A}_{\mathrm{I}}+\sigma_{\mathrm{GB}} \mathrm{A}_{\mathrm{GB}}
$$

where $A_{I}$ is the area fraction of grain interior, and $A_{G B}$ is the area fraction of grain boundary region. As the grain size of the polycrystal decreases, the $\mathrm{A}_{\mathrm{GB}}$ for the constant width grain boundary region increases resulting in an increase in the yield strength of the polycrystal. Equation (2.11) can be rewritten for square grains provided the grain boundary width $\left(\mathrm{w}_{\mathrm{GB}}\right)$ is much smaller than the grain size $(\mathrm{d})$ as

$$
\sigma_{\mathrm{y}}=\sigma_{\mathrm{I}}+\frac{4 \mathrm{w}_{\mathrm{GB}}}{\mathrm{d}}\left(\sigma_{\mathrm{GB}}-\sigma_{\mathrm{I}}\right)
$$

where $\mathrm{d}$ is the length of the square's side. Thus, the composite model has a natural $\mathrm{d}^{-1}$ dependence; $\mathrm{a} \mathrm{d}^{-1 / 2}$ dependence can only be obtained if $\mathrm{w}_{\mathrm{GB}}$ and $\sigma_{\mathrm{GB}}$ vary with $\mathrm{d}(\mathrm{Fu}$ et al., 2001).

Thompson et al. (1973) introduced a microstructure sensitive composite model based on Ashby's (1970) concept of statistically stored dislocations (SSDs) and geometrically necessary dislocations (GNDs). They assumed that the SSD density $\left(\rho^{\text {SSD }}\right)$ was proportional to the inverse SSD slip length and that the GND density $\left(\rho^{\mathrm{GND}}\right)$ was proportional to the inverse square root of the grain size. These assumptions led to the following equation for flow stress

$$
\sigma_{\mathrm{y}}=\sigma_{0}+\left(\frac{\mathrm{c}_{\mathrm{TH} 1}}{\lambda^{\mathrm{SSD}}}\right) \mathrm{A}_{\mathrm{I}}+\left(\frac{\mathrm{c}_{\mathrm{TH} 2}}{\sqrt{\mathrm{d}}}\right) \mathrm{A}_{\mathrm{GB}}
$$

where $\mathrm{c}_{\mathrm{TH} 1}$, and $\mathrm{c}_{\mathrm{TH} 2}$ are material constants. From Eq. (2.13), it follows that the GND based term dominates at small strains and the SSD based term dominates at large strains. 
The value of using local models to investigate length scale behavior is limited because the length scale dependence must be known a priori. In Eqs. (2.12) and (2.13), the $\frac{1}{\mathrm{~d}}$ and $\frac{1}{\sqrt{\mathrm{d}}}$ grain size dependence is specified in the yield strength constitutive equation. When the origin of the length scale in the local model is not based on micromechanics at much finer scales (as in the case of Eq. 2.13), then the local model is not a useful tool for understanding length scale phenomena. Local models with appropiatly defined lengh scale parameters can be used as an intermediate step between length scale independent local models and non-local models.

\subsubsection{Modeling Length Scale Phenomena with Non-Local Models}

Local models do not naturally account for experimentally observed length-scaledependent phenomena because the local description of the continuum breaks down when the representative scale of interest approaches the dominat material length scale. When this happens, researchers have recognized that the state of a point may depend on the states of points in some surrounding neighborhood. Models that consider such dependence are called non-local models.

To capture non-local effects, two types of models have been proposed: one involving gradients of internal variables and the other involving spatial integrals. By definition, the gradient of a variable depends on the value of this variable at the point of interest as well as the values in an infinitesimal neighborhood around this point. Thus, it includes information about the state of the material outside the point itself. Nevertheless, rigorously speaking, gradients are still defined at a point and, therefore, the state of a point is still determined only by the variables at this particular point. While some 
consider such models local, others, like Bazant and Jirasek (2002) classify models of this type as "weakly" non-local.

Integral non-local models differ from gradient models in that the state of a point is dependent on the on the states of all other points in the body not just the infinitesimal neighborhood around the point of interest. This dependence over the entire body is characterized by a functional, usually an integral. Because the state of the entire body is considered, Bazant and Jirasek (2002) classify models of this type as "strongly" nonlocal. Regardless of the mathematical formulation, both the gradient and integral nonlocal models can account for microsturcutral heterogeneity and can model length-scaledependent phenomena.

\subsubsection{Gradient Modeling}

The Cossreat brothers (1909) proposed the first enriched continuum model that considered higher order stresses. In the Cosserat continuum, each material point has the usual three translational degrees of freedom and three additional rotational degrees of freedom. The addition of rotational degrees of freedom leads to higher order stresses, or moments. There was not much interest in such gradient models until the discovery of dislocations and their influence on the mechanical response of materials. The need to describe and model materials with microstructure brought elastic gradient models to the forefront in the 1960s.

Toupin (1962) and Mindlin and Tiersten (1962) pioneered much of the work in the 1960s in gradient elasticity. They both developed couple-stress theories of elasticity 
by including rotation gradients in the free energy. In these gradient elasticity theories, stress was defined in the usual manner as

$$
\mathbf{t}=\boldsymbol{\sigma} \cdot \mathbf{n}
$$

where $\mathbf{t}$ is a force vector, $\boldsymbol{\sigma}$ is the Cauchy stress tensor, and $\mathbf{n}$ is a normal vector to the surface upon which the traction is applied. The couple-stress was defined in a similar manner, i.e.,

$$
\mathbf{c}=\underset{2}{\mathbf{v} \cdot \mathbf{n}}
$$

where $\mathbf{c}$ is a couple vector, and $\underset{2}{\mathbf{v}}$ is a $2^{\text {nd }}$ order couple stress tensor. Development of gradient elastic theories continued throughout the 1960s by a number of different researchers. Toupin (1964) extended the gradient elasticity framework to include stretch gradients as well as rotational gradients. Green and Rivlin (1964) established a framework that could include gradients of any order. Efforts were made to apply these theories to predict phenomena for elastic solids such as stress concentrations at holes (Mindlin, 1963), bending stiffness of thin beams (Koiter, 1964), and surface tension (Mindlin, 1965). In addition, the rotational gradients were related to material curvatures and Nye's dislocation tensor.

Dillion and Kratchovil (1970) extended gradient theories, similar in form to those of Toupin and Mindlin, to plasticity. Aifantis (1984) developed a gradient plasticity model that was based on gradient dependent dislocation evolution equations. However, it was not until the early 1990s with the phenomenological plastic strain gradient theory of Fleck and Hutchinson (1993) and Fleck et al. (1994) that gradient plasticity models became popular. Since then, a large number of gradient models have been proposed. Generally, the gradient plasticity models can be classified into two groups: (1) models 
that introduce gradient variables as new independent state variables, and (2) models that introduce gradient dependent evolution equations that influence hardening.

\subsection{Gradient Plasticity with Gradient State Variables}

Dillion and Kratchovil (1970) developed a gradient plasticity model based on the assumption that the free energy density depended on, among other variables, strain, the first gradient of strain, and the second gradient of strain. Since these terms also appear in the internal energy balance, the second law of thermodynamics requires that there be a stress conjugate to each of the three strain measures included in the free energy density. The stresses conjugate to each of the strain gradients were termed higher order stresses.

Using the thermodynamic framework developed by Dillion and Kratchovil (1970), Fleck and Hutchinson $(1993,1997)$ and Fleck et al. (1994) developed a phenomenological strain gradient theory. The model developed in Fleck and Hutchinson (1993) and Fleck et al. (1994) considered the effect of rotation gradients on the material response. In this model, a curvature tensor $(\boldsymbol{\chi})$ was defined as

$$
\chi_{\mathrm{ni}} \equiv \theta_{\mathrm{n}, \mathrm{i}}=\frac{1}{2} e_{\mathrm{njk}} \mathrm{u}_{\mathrm{k}, \mathrm{ji}}=e_{\mathrm{njk}} \varepsilon_{\mathrm{ij}, \mathrm{k}}
$$

where $e_{\mathrm{nkj}}$ is the permutation tensor, and $\boldsymbol{\theta}$ is the rotation vector associated with a

displacement field $\mathbf{u}$. The $2^{\text {nd }}$ order couple stress conjugate to $\chi \underset{2}{\boldsymbol{i}} \underset{\mathbf{v}}{\mathbf{v}}$. Thus the increase in elastic stored energy (w) due to deformation is

$$
\delta \mathrm{w}=\sigma^{\prime}: \delta \varepsilon+\mathbf{v}_{2}^{\prime}: \delta \chi
$$


where $\sigma^{\prime}$ and $\mathbf{v}_{2}^{\prime}$ are the deviatoric parts of $\boldsymbol{\sigma}$ and $\underset{2}{\mathbf{v}}$ respectively. In addition Fleck et al. (1994) proposed a relationship between the plastic rotation gradient $\left(\chi^{\mathbf{p}}\right)$ and $\rho^{\mathrm{GND}}$ via

$$
\rho^{\mathrm{GND}}=\frac{\chi^{\text {eff }}}{\mathrm{b}}
$$

where $\chi^{\text {eff }}=\sqrt{\frac{2}{3} \chi^{\mathbf{p}}: \chi^{\mathbf{p}}}$. They noted that $\chi^{\mathbf{p}}$ can be a meaningful measure of GNDs "when no crystal structure is embedded in the material since it is defined in terms of plastic strain $\left(\boldsymbol{\varepsilon}^{\mathbf{p}}\right)$ and not the slip tensor $\gamma . "$

Fleck and Hutchinson (1997) expanded the previous gradient theories (Fleck and Hutchinson, 1993; Fleck et al., 1994) to include a general strain gradient tensor ( $\eta$ ) that included slip gradients $\left(\gamma_{\mathrm{ij}, \mathrm{k}}\right)$, rotation gradients $\left(\mathrm{R}_{\mathrm{ij}, \mathrm{k}}\right)$, and elastic strain gradients $\left(\varepsilon_{\mathrm{ij}, \mathrm{k}}^{\mathrm{e}}\right)$

$$
\eta_{\mathrm{jki}} \equiv \mathrm{u}_{\mathrm{i}, \mathrm{jk}}=\gamma_{\mathrm{ij}, \mathrm{k}}+\mathrm{R}_{\mathrm{ij,k}}+\varepsilon_{\mathrm{ij}, \mathrm{k}}^{\mathrm{e}}
$$

The higher order stress conjugate to $\eta$ was the third order couple stress $\underset{3}{\mathbf{v}}$ such that the increase in w due to deformation was

$$
\delta \mathrm{w}=\sigma^{\prime} \delta \varepsilon+\mathbf{v}_{3}^{\prime} \delta \eta
$$

where $\mathbf{v}_{3}^{\prime}$ is the deviatoric part of $\underset{3}{\mathbf{v}}$. Within this model, a unique solution is found once 6 independent boundary conditions are prescribed: the three usual traction-velocity boundary conditions and three higher-order boundary conditions. These higher order boundary conditions are a weak spot in this approach since these boundary conditions are not always clear and straightforward to implement. Fleck and Hutchinson (1997) also defined $\rho^{\mathrm{GND}}$ in terms of an effective strain gradient $\left(\eta^{\text {eff }}\right)$

$$
\rho^{\mathrm{GND}}=\frac{\eta^{\text {eff }}}{\mathrm{b}}
$$


where $\eta^{\text {eff }}=\sqrt{\mathrm{c}_{\mathrm{FH} 1} \eta_{\mathrm{iik}} \eta_{\mathrm{jjk}}+\mathrm{c}_{\mathrm{FH} 2} \eta_{\mathrm{ijk}} \eta_{\mathrm{ijk}}+\mathrm{c}_{\mathrm{FH} 3} \eta_{\mathrm{ijk}} \eta_{\mathrm{kji}}}$. The three constants $\left(\mathrm{c}_{\mathrm{FH} 1}, \mathrm{c}_{\mathrm{FH} 2}\right.$, and $\mathrm{c}_{\mathrm{FH} 3}$ ) scaled the three quadratic invariants of the strain gradient tensor $\eta$. Fleck and Hutchinson (2001) recast their strain gradient theory using plastic strain rather than the total strain. They pointed out "The previous Fleck-Hutchinson (1997) deformation formulation incorrectly introduces a dependence on strain gradients in the linear elastic range".

Gao et al. (1999a) developed a "mechanism-based strain gradient plasticity" (MSG) model with a mathematical structure similar to the Fleck and Hutchinson (1997) model, but based on linking the microscale concept of SSDs and GNDs to the mesoscale plastic strain and strain gradient variables. At the microscale, it was assumed that the flow stress obeys the Taylor equation modified to account for strain gradients

$$
\hat{\sigma}^{\mathrm{eff}}=\sigma_{\mathrm{y}} \sqrt{\left[f\left(\widehat{\varepsilon}^{\mathrm{eff}}\right)\right]^{2}+\lambda \eta^{\mathrm{eff}}}
$$

where $\widehat{\sigma}^{\text {eff }}$ and $\widehat{\varepsilon}^{\text {eff }}$ are a scalar stress and strain at the microscale, $f\left(\widehat{\varepsilon}^{\text {eff }}\right)$ defines the material response in the absence of GNDs, and $\lambda$ is the material length scale given by

$$
\lambda=3 C^{2}\left(\frac{G}{\sigma_{y}}\right)^{2} b .
$$

The microscale and mesoscale were linked by equating the work done at the microscale to the work done at the mesoscale

$$
\int_{\mathrm{V}_{\text {cell }}} \hat{\sigma}^{\prime} \delta \widehat{\varepsilon} \mathrm{d} V=\left(\boldsymbol{\sigma}^{\prime} \delta \varepsilon+\mathbf{v}_{3}^{\prime} \delta \eta\right) \mathrm{V}_{\text {cell }}
$$

where $\boldsymbol{\sigma}^{\prime}, \boldsymbol{\varepsilon}, \mathbf{v}_{3}^{\prime}$, and $\eta$ are the mesoscale stress and strain tensors; $\widehat{\boldsymbol{\sigma}}$ and $\widehat{\boldsymbol{\varepsilon}}$ are the microscale stress and strain tensors; and $\mathrm{V}_{\text {cell }}$ represents the "small" mesoscale volume. 
Using the MSG model, Gao et al. (1999b) observed an increased work hardening in the bending of thin beams and the torsion of thin wires similar to that observed experimentally (Stolken and Evans, 1998; Fleck et al., 1994). Yun et al. (2005) reformulated MSG plasticity by making the higher order stress conjugate to the gradient in plastic (rather than total) strain.

Bassani (2001) developed a gradient theory in which the strain gradient "enters the flow rules only through the instantaneous hardening rate". Consequently, this gradient theory did not have any higher order stresses unlike the theories based on the Fleck-Hutchinson model. In the Bassani model, the curl of the plastic strain gradient was used as a measure of GNDs

$$
\mathrm{g}_{\mathrm{ij}}=e_{\mathrm{jk}} \varepsilon_{\mathrm{il}, \mathrm{k}}^{\mathrm{P}}
$$

where $\mathbf{g}$ is Nye's dislocation tensor (See section 3.1 for details on Nye's dislocation tensor). An invariant of the dislocation tensor, $\left(\mathrm{g}^{\text {eff }}\right)^{2}=2 \mathbf{g}: \mathbf{g}$, was incorporated into a hardening matrix to account for the increase in hardening rate due to GNDs. Using a $\mathbf{J}_{2}$ flow theory with work hardening related to plastic strain and $\mathrm{g}^{\text {eff }}$, Bassani (2001) was able to reasonably reproduce the thin wire torsion results of Fleck et al. (1994).

\subsection{Plasticity with Gradient Dependent Evolution Equations}

Based on the work of Bammann and Aifantis (1982), Aifantis (1984) proposed a theoretical framework for models that could predict localization of microstructure and macroscopic deformation. Aifantis's approach was based on defining evolution equations for dislocation densities that included gradient terms. Aifantis suggested 
evolution equations that considered the competition of mobile $\left(\rho^{\mathrm{M}}\right)$ and immobile dislocation states $\left(\rho^{\mathrm{I}}\right)$ (Walgraef and Aifantis, 1985a+b)

$$
\begin{gathered}
\dot{\rho}^{\mathrm{I}}=\mathrm{g}\left(\rho^{\mathrm{I}}\right)-\mathrm{c}_{\mathrm{WA}_{1}} \rho^{\mathrm{I}}+\rho^{\mathrm{M}}\left(\rho^{\mathrm{I}}\right)^{2}+\mathrm{D}_{\mathrm{WA}}^{\mathrm{I}} \nabla^{2} \rho^{\mathrm{I}} \\
\dot{\rho}^{\mathrm{M}}=\mathrm{c}_{\mathrm{WA1}} \rho^{\mathrm{I}}-\rho^{\mathrm{M}}\left(\rho^{\mathrm{I}}\right)^{2}+\mathrm{D}_{\mathrm{WA}_{\mathrm{A}}}^{\mathrm{M}} \nabla^{2} \rho^{\mathrm{M}}
\end{gathered}
$$

where $\mathrm{c}_{\mathrm{WA} 1}$ is a constant, and $\mathrm{D}_{\mathrm{WA}}^{\mathrm{I}}$ and $\mathrm{D}_{\mathrm{WA}}^{\mathrm{M}}$ are diffusion-like parameters for immobile and mobile dislocations. The right hand side of Eqs. (2.26) and (2.27) represent various production and annihilation mechanisms for dislocations: mobile dislocations are generated but immediately immobilized as described by $\mathrm{g}\left(\rho^{\mathrm{I}}\right)$, immobile dislocations break free at a rate $c_{\mathrm{WAl}} \rho^{\mathrm{I}}$, mobile dislocations are immobilized at a rate $\rho^{\mathrm{M}}\left(\rho^{\mathrm{I}}\right)^{2}$, and $D_{I} \nabla^{2} \rho^{I}$ and $D_{M} \nabla^{2} \rho^{M}$ account for dislocation motion due to an effective diffusion process. Walgraef and Aifantis (1988) and Glazov and Laird (1995) each developed a dynamical model based on a linear stability analysis of Eqs. (2.26) and (2.27). They applied their dynamical model to metal fatigue, where they observed patterning in $\rho^{\mathrm{I}}$ that looked similar to persistent slip bands (PSBs).

Ananthakrishna (1993) developed a set of gradient based dislocation evolution equations to model the Portevin-Le Chatelier (PLC) effect in monotonically deformed metals. The Ananthakrishna model introduced three dislocation types: $\rho^{\mathrm{M}}, \rho^{\mathrm{I}}$, and dislocations surrounded by clouds of solute atoms $\left(\rho^{\mathrm{SA}}\right)$

$$
\begin{gathered}
\dot{\rho}^{\mathrm{M}}+\frac{\partial}{\partial \mathrm{x}_{\mathrm{i}}}\left(\rho^{\mathrm{M}} \mathrm{v}^{\mathrm{M}}\right)=\mathrm{c}_{\mathrm{AN} 1} \mathrm{v}^{\mathrm{M}} \rho^{\mathrm{M}}-\mathrm{c}_{\mathrm{AN} 2}\left(\rho^{\mathrm{M}}\right)^{2}-\mathrm{c}_{\mathrm{AN} 3} \rho^{\mathrm{M}} \rho^{\mathrm{I}}+\mathrm{c}_{\mathrm{AN} 4} \rho^{\mathrm{I}}-\mathrm{c}_{\mathrm{AN} 5} \rho^{\mathrm{M}} \\
\dot{\rho}^{\mathrm{I}}+\frac{\partial}{\partial \mathrm{x}_{\mathrm{i}}}\left(\rho^{\mathrm{I}} \mathrm{v}^{\mathrm{I}}\right)=\mathrm{c}_{\mathrm{AN} 0} \mathrm{c}_{\mathrm{AN} 2}\left(\rho^{\mathrm{M}}\right)^{2}-\mathrm{c}_{\mathrm{AN} 3} \rho^{\mathrm{M}} \rho^{\mathrm{I}}-\mathrm{c}_{\mathrm{AN} 4} \rho^{\mathrm{I}}+\mathrm{c}_{\mathrm{AN} 6} \rho^{\mathrm{SA}}
\end{gathered}
$$




$$
\dot{\rho}^{\mathrm{SA}}+\frac{\partial}{\partial \mathrm{x}_{\mathrm{i}}}\left(\rho^{\mathrm{SA}} \mathrm{v}^{\mathrm{SA}}\right)=\mathrm{c}_{\mathrm{AN} 5} \rho^{\mathrm{M}}-\mathrm{c}_{\mathrm{AN} 6} \rho^{\mathrm{SA}} .
$$

where $\mathrm{v}^{\mathrm{M}}, \mathrm{v}^{\mathrm{I}}$, and $\mathrm{v}^{\mathrm{SA}}$ are the dislocation velocities; $\mathrm{c}_{\mathrm{AN} 0}, \mathrm{c}_{\mathrm{AN} 1}, \mathrm{c}_{\mathrm{AN} 2}, \mathrm{c}_{\mathrm{AN} 3}, \mathrm{c}_{\mathrm{AN} 4}, \mathrm{c}_{\mathrm{AN} 5}$, and $\mathrm{c}_{\mathrm{AN} 6}$ are material constants. Like Eqs. (2.26) and (2.27), the right hand side of Eqs. (2.28) - (2.30) accounted for various dislocation production and annihilation mechanisms. Ananthakrishna (1993) developed a model that used stability analysis to solve Eqs. (2.28) - (2.30). This model exhibited serrated flow (PLC effect) over a range of strain rates. He also observed that this strain rate range was sensitive to the nature of the spatial derivative.

Sluys and Estrin (2000) implemented a dislocation evolution equation that had a Walgraef-Aifantis like diffusion term (see Eqs. (2.26) and (2.27)) into a crystal plasticity framework. This dislocation evolution equation was derived by combining the KocksMecking model (Estrin and Mecking, 1983) with the dislocation diffusion idea of Walgraef and Aifantis (1985b), i.e,

$$
\langle\dot{\rho}\rangle=\dot{\gamma}^{\alpha}\left(\mathrm{c}_{\mathrm{KM} 1} \sqrt{\langle\rho\rangle^{\beta}}-\mathrm{c}_{\mathrm{KM} 2}\langle\rho\rangle^{\alpha}\right)+\mathrm{D}_{\mathrm{SE}} \nabla^{2}\langle\rho\rangle^{\alpha} \quad \alpha \neq \beta
$$

where $\langle\rho\rangle^{\alpha}$ and $\langle\rho\rangle^{\beta}$ are the dislocation densities on the slip systems $\alpha$ and $\beta$ respectively, $\mathrm{c}_{\mathrm{KM} 1}$ is a constant related to athermal dislocation storage, $\mathrm{c}_{\mathrm{KM} 2}$ is a constant related to thermally activated recovery, and $\mathrm{D}_{\mathrm{SE}}$ is a constant related to dislocation 'diffusivity'. In their analysis of the $\langle\rho\rangle$ field, Sluys and Estrin (2000) noted that the positive $\mathrm{D}_{\mathrm{SE}} \nabla^{2}\langle\rho\rangle^{\alpha}$ term acted against the localization of $\langle\rho\rangle$ and worked to homogenize the $\langle\rho\rangle$ field. Within their crystal plasticity model, $\langle\rho\rangle$ affected the hardening of the material via Taylor's equation, Equ. (2.3). Sluys and Estrin (2000) used this model to 
capture shear banding, or strain localization, in a single crystal strip orientated for double slip under tension.

Acharya and Beaudoin (2000) developed a crystal plasticity model in which hardening was governed by a dislocation evolution equation that included the effects of lattice incompatibility. They defined a incompatibility tensor, $\Lambda$, as

$$
\Lambda=\left(\mathbf{F}^{\mathrm{e}^{-1}}\right)_{\mathrm{ij}, \mathrm{k}}-\left(\mathbf{F}^{\mathbf{e}^{-1}}\right)_{\mathrm{ik}, \mathrm{j}}
$$

where $\mathbf{F}^{\mathrm{e}}$ is the elastic deformation gradient. An effective incompatibility on each slip system a was represented by $\left(\Lambda^{\text {eff }}\right)^{\alpha}$ via

$$
\left(\Lambda^{\mathrm{eff}}\right)^{\alpha}=\sqrt{\left(\Lambda: \overline{\mathbf{n}}^{\alpha}\right) \cdot\left(\Lambda: \overline{\mathbf{n}}^{\alpha}\right)}
$$

where $\overline{\mathbf{n}}^{\alpha}$ is the unique skew-symmetric tensor defined by $\overline{\mathbf{n}}^{\alpha} \cdot \mathbf{a}=1 / 2 \mathbf{n}^{\alpha} \cdot \mathbf{a}$ for all vectors $\mathbf{a}$ and each slip plane normal $\mathbf{n}^{\alpha}$. Acharya and Beaudoin (2000) modified the Kocks-

Mecking model (Estrin and Mecking, 1983) to include the effects of $\left(\Lambda^{\text {eff }}\right)^{\alpha}$

$$
\langle\dot{\rho}\rangle=\sum_{\alpha}\left(\mathrm{c}_{\mathrm{AB} 0} \frac{\left(\Lambda^{\mathrm{eff}}\right)^{\alpha}}{\mathrm{b}}+\mathrm{c}_{\mathrm{KM} 1} \sqrt{\langle\rho\rangle}-\mathrm{c}_{\mathrm{KM} 2}\langle\rho\rangle\right)\left|\dot{\gamma}^{\alpha}\right|
$$

where $\mathrm{c}_{\mathrm{AB} 0}$ is a material constant. The $\mathrm{c}_{\mathrm{AB} 0} \frac{\left(\Lambda^{\mathrm{eff}}\right)^{\alpha}}{\mathrm{b}}$ term represented storage of dislocations due to lattice incompatibility. In Equ. (2.34), dislocations needed for compatibility also increase the overall dislocation density for a given slip increment by acting as obstacles to glide dislocations. The Taylor equation then relates $\langle\rho\rangle$ to the work hardening of the material. Acharya and Beaudoin (2000) applied their crystal plasticity model to nickel polycrystals with various grain sizes and observed a 
relationship between flow stress at various strains and grain size as $\sigma_{\mathrm{y}}(\varepsilon) \propto \mathrm{d}^{-\frac{4}{5}}$. Beaudoin et al. (2000) extended this model to account for temperature and strain rate effects. They observed good agreement between stress-strain curves predicted by the model and experimental stress-strain curves for the compression of silver polycrystals at different temperatures and strain rates.

Evers et al. (2004a) created a crystal plasticity model in which GND production was related to slip gradients on each slip system. In the Evers model, there were 18 basic dislocation types (12 edge and 6 screws) that could be used as building blocks to construct any dislocation structure within an FCC material. The GND evolution for each of these dislocation types $(\zeta)$ was

$$
\rho^{\mathrm{GND}-\zeta}=\rho_{\mathrm{t}=0}^{\mathrm{GND}-\zeta}-\frac{1}{\mathrm{~b}} \sum_{\alpha} \mathrm{d}_{\mathrm{e}}^{\alpha \zeta}\left(\nabla \gamma^{\alpha} \cdot \mathbf{S}^{\alpha}\right)+\frac{1}{\mathrm{~b}} \sum_{\alpha} \mathrm{d}_{\mathrm{s}}^{\alpha \zeta}\left(\nabla \gamma^{\alpha} \cdot\left[\mathbf{S}^{\alpha} \times \mathbf{M}^{\alpha}\right]\right)
$$

where $\rho^{\mathrm{GND}-\zeta}$ is the GND density of type $\zeta, \rho_{\mathrm{t}=0}^{\mathrm{GND}-\zeta}$ is the initial GND density of type $\zeta, \mathbf{S}^{\alpha}$ is the slip direction vector in the reference configuration of slip system $\alpha, \mathbf{M}^{\alpha}$ is the normal to slip the slip plane in the reference configuration of slip system $\alpha$, and $\mathrm{d}_{\mathrm{e}}^{\zeta \alpha}$ and $\mathrm{d}_{\mathrm{s}}^{\zeta \alpha}$ describe the relation between edge (e) and screw (s) dislocation densities of type $\zeta$ and slip system $\alpha$. SSD evolution was based on a balance between accumulation $\left(1^{\text {st }}\right.$ term) and annihilation $\left(2^{\text {nd }}\right.$ term) rates

$$
\dot{\rho}^{\mathrm{SSD}-\zeta}=\frac{1}{\mathrm{~b}}\left(\frac{1}{\mathrm{~d}_{\mathrm{e}}^{\alpha \zeta} \mathrm{c}_{\mathrm{EV} 1}^{\alpha}}-2 \mathrm{y}_{\mathrm{c}} \rho^{\mathrm{SSD}-\zeta}\right) \mathrm{d}_{\mathrm{e}}^{\alpha \zeta}\left|\dot{\gamma}^{\alpha}\right|
$$

where $\mathrm{c}_{\mathrm{EV} 1}^{\alpha}$ represents an average mobile SSD segment length on slip system $\alpha$, and $\mathrm{y}_{\mathrm{c}}$ is a critical annihilation length. The resistance to slip due to short-range obstacles (i.e. 
dislocation interactions that impede the motion of gliding dislocations) was accounted for with a Taylor equation based hardening law

$$
\tau^{\mathrm{CSS}-\alpha}=\mathrm{CGb} \sqrt{\sum_{\zeta} \mathrm{A}^{\alpha \zeta}\left|\rho^{\mathrm{SSD}-\zeta}\right|+\sum_{\zeta} \mathrm{A}^{\alpha \zeta}\left|\rho^{\mathrm{GND}-\zeta}\right|}
$$

where $\mathrm{A}^{\alpha \zeta}$ accounts for the strength of various dislocation junctions. In addition, a nonuniform GND distribution led to a back stress that acted counter to the resolved shear stress of a slip system. The back stress due to edge $\left(\tau_{\mathrm{e}}^{\alpha}\right)$ and screw dislocations $\left(\tau_{\mathrm{s}}^{\alpha}\right)$ on slip system $\alpha$ was defined as

$$
\tau_{\mathrm{s}}^{\alpha}=\frac{\mathrm{Gbr}^{2}}{4} \sum_{\zeta} \mathrm{d}_{\mathrm{e}}^{\alpha \zeta}\left(\nabla \rho^{\mathrm{GND}-\zeta} \cdot\left[\mathbf{S}^{\alpha} \times \mathbf{M}^{\alpha}\right]\right) \quad \tau_{\mathrm{e}}^{\alpha}=-\frac{\mathrm{Gbr}^{2}}{8(1-v)} \sum_{\zeta} \mathrm{d}_{\mathrm{e}}^{\alpha \zeta}\left(\nabla \rho^{\mathrm{GND}-\zeta} \cdot \mathbf{S}^{\alpha}\right)
$$

where $\mathrm{r}$ is the radius over which the back stress is felt. The total back stress, $\tau_{\mathrm{b}}$, was then a projection of $\tau_{\mathrm{e}}$ and $\tau_{\mathrm{s}}$ onto the Schmid tensor, $\mathbf{P}^{\alpha}$, via

$$
\tau_{\mathrm{b}}=\sum_{\alpha}\left(\tau_{\mathrm{e}}^{\alpha}+\tau_{\mathrm{b}}^{\alpha}\right)\left(\mathbf{P}^{\alpha}+\mathbf{P}^{\alpha^{\mathrm{T}}}\right)
$$

Evers et al. (2004a) validated this model by simulating a constrained strip of single crystal copper oriented for double slip subjected to a simple shear deformation. In these simulations, they observed the formation of boundary layers (layers of material where slip is reduced) as well as an effect of strip height on the stress-strain response.

Evers et al. (2004b) applied the model in Evers et al. (2004a) to polycrystalline materials. In polycrystalline materials, they associated $\rho_{0}^{\mathrm{GND}-\zeta}$ with the initial grain boundary dislocation density field. Evers et al. (2004b) proposed a geometric argument based on the lattice mismatch between two grains to estimate $\rho_{0}^{\mathrm{GND}-\zeta}$. This geometric argument is illustrated in Figure 2.15. 


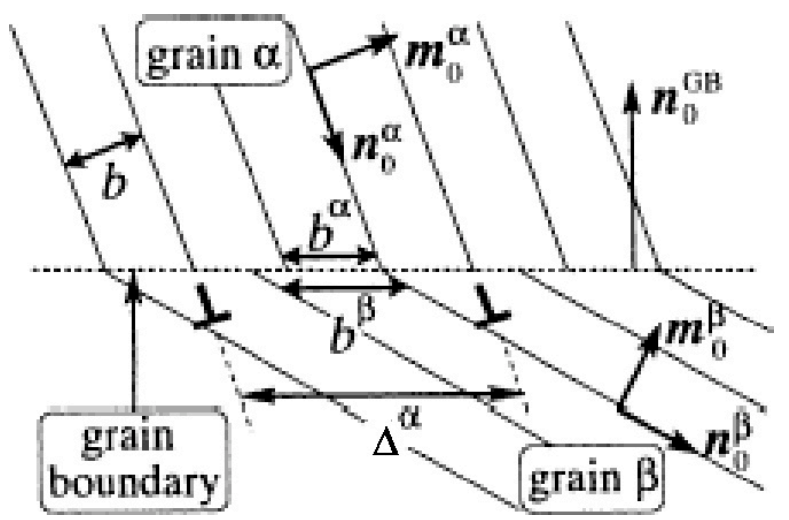

Figure 2.15 - The lattice mismatch at the grain boundary that determined the initial GND density (Evers et al., 2004b)

The misfit length, $\Delta^{\alpha}$, between a slip system in grain $\alpha$ and its associated slip system in grain $\beta$ was related to $\rho_{0}^{\mathrm{GND}-\zeta}$ by

$$
\rho_{\mathrm{t}=0}^{\mathrm{GND}-\zeta} \equiv \mathrm{d}_{\mathrm{e}}^{\alpha \zeta} \frac{1}{\left(\Delta^{\alpha}\right)^{2}}=\mathrm{d}_{\mathrm{e}}^{\alpha \zeta}\left(\frac{1}{\mathrm{~b}^{\alpha}}-\frac{1}{\mathrm{~b}^{\beta}}\right)^{2}
$$

Evers et al. (2004b) investigated the grain size dependence of a 12 grain polycrystal under a plane stress tensile load. They observed that the GND field was strongly size dependent: as grain size decreased $\rho^{\mathrm{GND}}$ increased. This size dependence of $\rho^{\mathrm{GND}}$ led to a grain size effect on the yield stress with a $\mathrm{d}^{-1.19}$ dependence, which does not agree with the generally accepted $\mathrm{d}^{-0.5}$.

\subsubsection{Non-local Integral Modeling}

In the 1960 s the non-local integral approach was extended to solid mechanics by Rogula (1965) and subsequently refined by Eringen (1966), Kroner (1966), and Edelen 
(1969) among others. As with gradient models, non-local integral models were proposed as a way to extend continuum theories to smaller length scales where classical continuum theories break down. It is interesting to note that at very small length scales, the interatomic potentials used in atomistic modeling exhibit a similar non-locality. Using this non-local framework at the continuum level, researchers were able to approximate the dispersion of short elastic waves, improve the description of interactions between crystal defects, and smooth the unrealistic stress singularities at the crack tip.

\subsection{Non-Local Integral Elasticity Models}

The non-local elasticity theories proposed by Edelen et al. (1971), Eringen (1972), and Eringen and Edelen (1972) placed no restrictions on which variables could have non-local character. Thus non-local variables could be included in the equilibrium equation (non-local forces) or in any of the balance laws (non-local momentum, nonlocal energy, non-local entropy). Unfortunately, these theories were too complicated to calibrate and verify experimentally, let alone to apply to any real problems. Therefore, these theories were simplified to a form where only the stress-strain relation was treated as non-local, leaving the rest of the problem in its standard form.

In this simplified form of non-local elasticity (Eringen and Kim, 1974; Eringen et al., 1977), the constitutive equation for stress, in its most general form, was

$$
\sigma(\mathbf{x})=\int_{V} F(\mathbf{x}, \xi, \varepsilon(\xi)) d \xi
$$


where $\boldsymbol{\xi}$ represent the local coordinate system centered at $\mathbf{x}$, and $F(\mathbf{x}, \boldsymbol{\xi}, \boldsymbol{\varepsilon}(\boldsymbol{\xi}))$ is the kernel of the integral operator. With a linear integral operator in Equation (2.41), the non-local constitutive equation for stress simplified to

$$
\boldsymbol{\sigma}(\mathbf{x})=\int_{\mathrm{V}} \underset{\sim}{\boldsymbol{L}}(\mathbf{x}, \boldsymbol{\xi}) \boldsymbol{\varepsilon}(\boldsymbol{\xi}) \mathrm{d} \boldsymbol{\xi}
$$

where $\underset{\sim}{\boldsymbol{L}}(\mathbf{x}, \boldsymbol{\xi})$ is the kernel of the elastic integral operator. It is often assumed that the elastic stiffness coefficients decay in the same manner. Thus $\underset{\sim}{\boldsymbol{L}}(\mathbf{x}, \boldsymbol{\xi})$ can be rewritten as a constant times an attenuation function, $\Theta(\mathbf{x}, \xi)$, describing how the stiffness coefficients decay

$$
\underset{\sim}{\boldsymbol{L}}(\mathbf{x}, \boldsymbol{\xi})=\underset{\sim}{\boldsymbol{L}}(\mathbf{x}) \Theta(\mathbf{x}, \xi)
$$

The linear elastic non-local constitutive relation can then be written as

$$
\boldsymbol{\sigma}(\mathbf{x})=\underset{\sim}{\boldsymbol{L}}(\mathbf{x}) \int_{\mathrm{V}} \Theta(\mathbf{x}, \boldsymbol{\xi}) \boldsymbol{\varepsilon}(\boldsymbol{\xi}) \mathrm{d} \xi
$$

If the attenuation function in Equation (2.44) is assumed to have the form of a $\delta$-function, then classical local elasticity is recovered

$$
\sigma(\mathbf{x})=\underset{\sim}{\boldsymbol{L}}(\mathbf{x}) \int_{\mathrm{V}} \delta(\mathbf{x}-\xi) \varepsilon(\xi) \mathrm{d} \xi=\underset{\sim}{\boldsymbol{L}}(\mathbf{x}) \boldsymbol{\varepsilon}(\mathbf{x})
$$

In non-local theories, the attenuation function plays a key role, which makes the form of this function very important. Physically, the hope is that some physics related to the non-local variable can be embedded in $\Theta(\mathbf{x}, \xi)$. Unfortunately, defining a physically meaningful attenuation function is often times difficult. Mathematically, it is often time advantageous that $\Theta(\mathbf{x}, \xi)$, does not alter a uniform field, i.e.

$$
\int_{\mathrm{V}} \Theta(\mathbf{x}, \boldsymbol{\xi}) \mathrm{d} \xi=1
$$

Even though there are many possible functions that satisfy Equation (2.46), the non-local 
attenuation function is often assumed to have the form of a Gaussian distribution

$$
\Theta(r)=(\omega \sqrt{2 \pi})^{-\operatorname{Dim}} \exp \left(-\frac{r^{2}}{2 \omega^{2}}\right)
$$

where Dim is the number of dimensions, and $\omega$ is a parameter with length dimensions. The problem with this form of the attenuation function is that the function approaches zero but never equals zero. Thus all points in the body, regardless of how far apart they are, interact with each other, which is computationally inefficient. Bazant and Ozbolt (1990) proposed a more computationally efficient attenuation function that takes the form of a polynomial bell-shaped function.

\subsection{Non-Local Integral Plasticity Models}

Eringen (1981) generalized his non-local elasticity theory to include plasticity. He developed a strain space plasticity model in which the stress at $\mathbf{x}$ was dependant on the elastic strain in the surrounding material via

$$
\boldsymbol{\sigma}(\mathbf{x})=\underset{\sim}{\boldsymbol{L}}(\mathbf{x}) \int_{\mathrm{V}}\left(\varepsilon(\xi)-\varepsilon_{\mathrm{p}}(\xi)\right) \mathrm{d} \xi
$$

Equ. (2.48) essentially states that stress is conjugate to a non-local elastic strain. Thus, this approach gives the model non-local character in both the elastic and plastic regimes, which may or may not be desirable. Eringen (1983) recast this non-local plasticity theory in stress-space. Eringen $(1981,1983)$ did not apply either of his non-local plasticity theories to any plasticity problems. 
Bazant and Lin (1988) advanced a non-local plasticity model to describe strain softening in materials. In their flow plasticity theory, they made only the one variable non-local: plastic strain. The non-local plastic strain variable, $\left\{\boldsymbol{\varepsilon}^{\mathrm{P}}\right\}$, was defined as

$$
\left\{\boldsymbol{\varepsilon}^{\mathbf{p}}\right\}(\mathbf{x})=\frac{1}{V_{\text {cell }}} \int \exp \left(-\pi^{2}\left(\frac{\mathbf{x}-\boldsymbol{\xi}}{\omega}\right)^{2}\right) \boldsymbol{\varepsilon}^{\mathbf{p}}(\boldsymbol{\xi}) \mathrm{d} \boldsymbol{\xi}
$$

where \{\} denote a non-local integral quantity, and $V_{\text {cell }}=\int \exp \left(-\pi^{2}(\mathbf{x}-\xi / \omega)^{2}\right) d \xi$. The stress-strain equation in this model was

$$
\boldsymbol{\sigma}(\mathbf{x})=\underset{\sim}{\boldsymbol{L}}(\mathbf{x})\left(\varepsilon(\mathbf{x})-\left\{\boldsymbol{\varepsilon}^{\mathbf{p}}\right\}(\mathbf{x})\right)
$$

in which total strain remained local and plastic strain was non-local. The yield function was written with local variables only. This non-local model was successfully used with the finite element method (FEM) to model tensile as well as compressive strain softening. In particular, Bazant and Lin (1988) modeled compression softening of soil around tunnel sides.

Stromberg and Ristinmaa (1996) and Nilson (1997) developed flow plasticity theories of plasticity with non-local integral variables. In both models, a non-local hardening variable $\{\kappa\}$ was defined as

$$
\{\kappa\}(\mathbf{x})=\frac{1}{\mathrm{~V}_{\text {cell }}} \int \exp \left(-\pi^{2}\left(\frac{\mathbf{x}-\boldsymbol{\xi}}{\omega}\right)^{2}\right) \kappa(\boldsymbol{\xi}) \mathrm{d} \boldsymbol{\xi}
$$

and $V_{\text {cell }}=\int \exp \left(-\pi^{2}(\mathbf{x}-\xi / \omega)^{2}\right) d \xi .\{\kappa\}$ entered the material model through the yield function $(f)$

$$
f=\sigma^{\text {eff }}-\sigma_{\mathrm{y}}-h(\{\kappa\})
$$


where $\sigma^{\text {eff }}$ is an effective stress and $h$ is the hardening function. The difference between these two models lay in the constitutive stress-strain equation. Stromberg and Ristinmaa (1996) used the local version

$$
\sigma(\mathbf{x})=\underset{\sim}{L}(\mathbf{x})\left(\varepsilon(\mathbf{x})-\varepsilon^{\mathbf{p}}(\mathbf{x})\right)
$$

while Nilson (1997) used a version based on a non-local integral plastic strain measure

$$
\boldsymbol{\sigma}(\mathbf{x})=\underset{\sim}{\boldsymbol{L}}(\mathbf{x})\left(\boldsymbol{\varepsilon}(\mathbf{x})-\left\{\boldsymbol{\varepsilon}^{\mathbf{p}}\right\}(\mathbf{x})\right)
$$

where the definition of $\left\{\boldsymbol{\varepsilon}^{\mathbf{p}}\right\}$ was

$$
\left\{\boldsymbol{\varepsilon}^{\mathbf{p}}\right\}(\mathbf{x})=\frac{1}{\mathrm{~V}_{\text {cell }}} \int \mathrm{e}^{-\pi(\mathbf{x}-\boldsymbol{\xi})^{2} / \omega^{2}} \boldsymbol{\varepsilon}^{\mathbf{p}}(\boldsymbol{\xi}) \mathrm{d} \boldsymbol{\xi}
$$

Both models were applied to a strain softening material. Stromberg and Ristinmaa (1996) implemented their model in FEM and observed a well-defined localization region that was mesh independent. Nilson (1997) derived an analytical solution for the 1D strain softening problem. He demonstrated that it is possible to entirely define the width of the localized zone by the parameter $\omega$. Borino and Polizzotto (1999) and Nilson (1999) later pointed out that the model proposed in Nilson (1997) was not consistent with the postulate of maximum plastic dissipation.

Gao and Huang (2001) proposed a non-local integral model that used Fleck and Hutchinson's (1997) framework to connect plastic strain gradients with dislocations and hardening. The Gao-Huang model was based on a non-local integral approximation of the strain gradient

$$
\left\{\varepsilon_{\mathrm{ij}, \mathrm{k}}\right\}(\mathbf{x})=\left(\int_{\mathrm{Vcell}}\left(\varepsilon_{\mathrm{ij}}(\mathbf{x}+\xi)-\varepsilon_{\mathrm{ij}}(\mathbf{x})\right) \xi_{\mathrm{m}} \mathrm{d} \xi\right) \cdot\left(\int_{\mathrm{Vcell}} \xi_{\mathrm{k}} \xi_{\mathrm{m}} \mathrm{d} \xi\right)^{-1}
$$


Representing the strain gradient as a non-local integral of strains resulted in a constitutive model free of higher order stresses and strains. $\left\{\varepsilon_{\mathrm{ij}, \mathrm{k}}\right\}$ was then directly related to $\rho^{\mathrm{GND}}$, which affected the work-hardening of the material via the Taylor's equation. $\left\{\varepsilon_{\mathrm{ij}, \mathrm{k}}\right\}$ and its affect on $\rho^{\mathrm{GND}}$ were incorporated into both a flow plasticity model and a deformation plasticity model. With these models, Gao and Huang (2001) simulated the depth dependence of hardness during nanoindentation (McElhaney et al., 1998), as well as the increased work hardening observed in torsion (Fleck et al., 1994) and bending (Stolken and Evans, 1998).

Clayton and McDowell (2003a) developed a non-local plasticity model based on volume averaging kinematic quantities, i.e. local deformation gradients. The volume average of the local plastic deformation field $\left(\mathbf{F}^{\mathbf{P}}\right)$ was represented by $\left\{\mathbf{F}^{\mathbf{P}}\right\}$

$$
\left\{\mathbf{F}^{\mathbf{P}}\right\} \equiv \frac{1}{\mathrm{~V}_{\text {cell }}} \int_{\mathrm{V}_{\text {cell }}} \mathbf{F}^{\mathbf{P}} \mathrm{dV}
$$

In addition, they introduced an additional non-local integral variable, the mesoincompatibility tensor $\left\{\mathbf{F}^{\mathbf{I}}\right\}$

$$
\left\{\mathbf{F}^{\mathrm{I}}\right\} \equiv\left(\frac{1}{\mathrm{~V}_{\text {cell }}} \int_{\mathrm{V}_{\text {cell }}} \mathbf{F}^{\mathrm{e}} \mathbf{F}^{\mathbf{P}} \mathrm{dV}\right)\left\{\mathbf{F}^{\mathbf{P}}\right\}^{-1}
$$

Within the Clayton-McDowell model, the total deformatin gradient was multiplicatively decomposed into three deformation gradient components

$$
\{\mathbf{F}\}=\left\{\mathbf{F}^{\mathrm{e}}\right\}\left\{\mathbf{F}^{\mathrm{I}}\right\}\left\{\mathbf{F}^{\mathrm{P}}\right\}
$$

Since $\left\{\mathbf{F}^{\mathbf{P}}\right\}$ and $\left\{\mathbf{F}^{\mathbf{I}}\right\}$ affected the strain state with the material body, the non-local effects captured by $\left\{\mathbf{F}^{\mathbf{P}}\right\}$ and $\left\{\mathbf{F}^{\mathbf{I}}\right\}$ propagated through the material model and affect the material bodies stress-strain response. This volume averaging framework was embedded into a 
crystal plasticity model, and used with FEM to model the tensile, compressive, and shear response of copper polycrystals. The simulation results showed concentrations of effective stress and elastic energy density in the vicinity of high-angle boundaries and triple points. Clayton and McDowell (2003a) hypothesized that these areas may serve as damage initiation sites within the material. 


\section{CHAPTER 3}

\section{CRYSTAL PLASTICITY THEORY}

The history of plasticity theory dates back to 1864 when Tresca published his yield

criterion based on experimental results on metal punching and extrusion process. Since then a tremendous amount of progress has been made in the development of mathematical theories of plasticity by researchers, such as Saint-Venant, Levy, von Mises, Hecky, and Prandtl. These mathematical theories are formulated to represent experimental observations with no real attempt to capture deformation mechanisms or deformation physics.

Starting in the 1930s, the growing awareness of the effect of microstructure on the mechanical response of metallic materials led researchers to develop more physically consistent extensions to the mathematical theory of plasticity. A number of different plasticity models based on deformation physics have been developed. Work by Schmid, Hill, Rice, and Asaro led to the theory of crystal plasticity. Crystal plasticity theory provided the framework for developing models that account for grain orientation, texture, and slip-system hardening. The two building blocks upon which all crystal plasticity theories and models are built are (1) Kinematics and (2) Kinetics and Constitutive Relations. While not a part of crystal plasticity theory, a third consideration, numerical integration, is also important. In non-local models with gradient terms (like $\chi^{\mathrm{pl}}, \eta, \Lambda$, and $\nabla \gamma$ from Chapter 2), a fourth consideration can also be important, namely the numerical approach used to determine the gradient quantity. This chapter contains a brief discussion of each of these four points. 


\subsection{Kinematics}

At the continuum level, the physical deformation of a material body can be expressed mathematically by the deformation gradient tensor, $\mathbf{F}$. The mathematical definition of $\mathbf{F}$ at a material point depends only on the initial and current position of that material point. In order to embed some material physics in $\mathbf{F}$, the total deformation gradient is decomposed into multiple components, where each component represents a different deformation mechanism or microstructural feature. There are an infinite number of ways in which the deformation gradient can be decomposed. One possibility is an additive decomposition into an elastic deformation gradient $\left(\mathbf{F}^{\mathbf{e}}\right)$ and a plastic deformation gradient $\left(\mathbf{F}^{\mathbf{P}}\right)$ according to (Nemat-Nasser, 1979)

$$
\mathbf{F}=\mathbf{F}^{\mathrm{e}}+\mathbf{F}^{\mathrm{P}}
$$

However, it is more common to use a multiplicative decomposition, like

$$
\begin{gathered}
\mathbf{F}=\mathbf{F}^{\mathrm{e}} \cdot \mathbf{F}^{\mathbf{P}} \quad \text { (Bilby } \text { et al. }, \text { 1955; Lee, 1969) } \\
\mathbf{F}=\mathbf{R}^{\mathrm{e}} \cdot \mathbf{F}^{\mathbf{P}} \quad \text { (Mathur and Dawson, 1989) } \\
\mathbf{F}=\mathbf{F}^{\theta} \cdot \mathbf{F}^{\mathrm{e}} \cdot \mathbf{F}^{\mathbf{P}}(\text { Yu } \text { et al. }, 1997)
\end{gathered}
$$

where $\mathbf{R}^{\mathbf{e}}$ is the elastic rotation tensor and $\mathbf{F}^{\theta}$ is a thermal deformation gradient. While both the additive and multiplicative decompositions are mathematically acceptable, there are criticisms in the literature on the physics of the additive elastic/plastic decomposition (Lee, 1981). Clayton and McDowell (2003b) proposed a hybrid approach to account for the effects of damage

$$
\mathbf{F}=\mathbf{F}^{\mathrm{e}} \cdot\left(\mathbf{F}^{\mathrm{m}}+\mathbf{F}^{\mathrm{d}}\right)
$$


where $\mathbf{F}^{\mathrm{m}}$ is the plastic deformation gradient associated with the bulk material and $\mathbf{F}^{\mathrm{d}}$ is the plastic deformation gradient associated with damage.

The complexity of the physical deformation process makes it possible to have a large number of terms in the deformation gradient decomposition in order to account for each deformation mechanism. However, each additional component adds a layer of complexity to the model making it uncommon for a model to have more than 3 components in the deformation gradient decomposition. In fact, most models are based on a 2-term decomposition. $\mathbf{F}^{\mathbf{e}} \cdot \mathbf{F}^{\mathbf{P}}$ is commonly used to model elastic-plastic behavior of metals. $\mathbf{F}^{\mathbf{P}}$ describes the irreversible deformation due to dislocation glide, and $\mathbf{F}^{\mathbf{e}}$ accounts for the stretching and rotation of the lattice. Note: $\mathbf{F}^{\mathbf{e}}$ does not necessarily represent a reversible elastic deformation. Another two term decomposition, $\mathbf{R}^{\mathbf{e}} \cdot \mathbf{F}^{\mathbf{P}}$, has been used to model texture evolution due to large plastic deformations. $\mathbf{R}^{\mathbf{e}}$ describes the change in orientation, while $\mathbf{F}^{\mathbf{P}}$ is again related to dislocation glide.

\subsubsection{Single Crystal $\mathbf{F}^{\mathrm{e}} \mathbf{F}^{\mathrm{P}}$}

The elastic-plastic multiplicative decomposition, Eq. (3.2), is commonly used to describe the mechanical response of single crystals. While in reality elastic and plastic deformations occur simultaneously, this deformation model decouples the elastic and plastic deformation processes resulting in the two-step deformation process shown in Figure 3.1. 


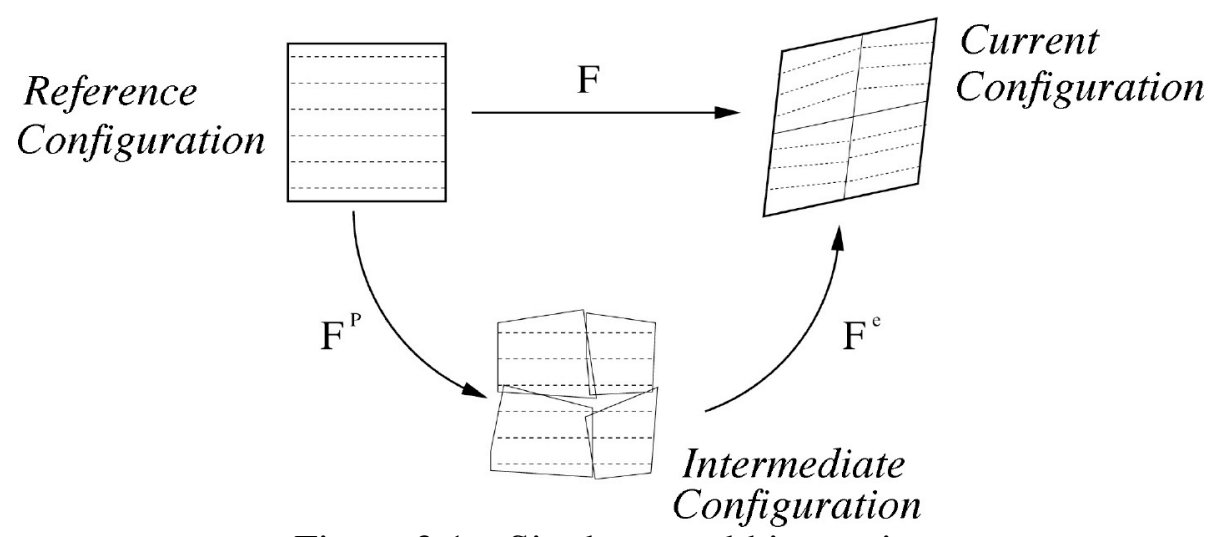

Figure 3.1 - Single crystal kinematics

Within the deformation model, the lattice is first sheared plastically as a result of dislocation glide as described by $\mathbf{F}^{\mathbf{P}}$. This dislocation motion produces a shape change but no lattice orientation change. The shape change described by $\mathbf{F}^{\mathbf{P}}$ can produce voids and overlaps in the intermediate configuration resulting in an incompatible, non-physical configuration. Compatibility is then restored in the current configuration when the lattice is then elastically stretched and rotated by $\mathbf{F}^{\mathbf{e}}$.

Mathematically, $\mathbf{F}, \mathbf{F}^{\mathbf{e}}$, and $\mathbf{F}^{\mathbf{P}}$ are mappings between the three configurations shown in Figure 3.1. By definition, each deformation gradient maps a differential line segment from one configuration to another via

$$
\begin{array}{rr}
\mathbf{F} \cdot \mathrm{d} \mathbf{X}=\mathrm{d} \mathbf{x} & \mathrm{d} \mathbf{X}=\mathbf{F}^{-1} \cdot \mathrm{d} \mathbf{x} \\
\mathbf{F}^{\mathbf{P}} \cdot \mathrm{d} \mathbf{X}=\mathrm{d} \hat{\mathbf{X}} & \mathrm{d} \mathbf{X}=\mathbf{F}^{\mathbf{P}^{-1}} \cdot \mathrm{d} \hat{\mathbf{X}} \\
\mathbf{F}^{\mathrm{e}} \cdot \mathrm{d} \hat{\mathbf{X}}=\mathrm{d} \mathbf{x} & \mathrm{d} \hat{\mathbf{X}}=\mathbf{F}^{\mathrm{e}^{-1}} \cdot \mathrm{d} \mathbf{x}
\end{array}
$$

where $d \mathbf{X}, \mathbf{d x}$, and $\mathrm{d} \hat{\mathbf{X}}$ are differential line segments in the reference, current, and intermediate configurations, respectively. The assumption that dislocation glide on crystallographic slip systems do not result in an orientation change means that lattice 
vectors are not altered by $\mathbf{F}^{\mathbf{P}}$. Thus the two lattice vectors that define a slip system, the slip direction vector and the slip plane normal vector, are unchanged between the reference and intermediate configurations, i.e.,

$$
\begin{aligned}
\mathbf{M}^{\alpha} & =\hat{\mathbf{m}}^{\alpha} \\
\mathbf{S}^{\alpha} & =\hat{\mathbf{s}}^{\alpha}
\end{aligned}
$$

where $\mathbf{S}^{\alpha}$ and $\mathbf{M}^{\alpha}$ are the slip direction vector and the slip plane normal vector for slip system $\alpha$ in the reference configuration, and $\hat{\mathbf{m}}^{\alpha}$ and $\hat{\mathbf{s}}^{\alpha}$ are the slip direction vector and the slip plane normal vector for slip system $\alpha$ in the intermediate configuration. As the crystal deforms elastically, these lattice vectors are rotated and stretched by $\mathbf{F}^{\mathrm{e}}$. These vectors are pushed forward from the intermediate configuration to the current configuration by $\mathbf{F}^{\mathbf{e}}$ according to

$$
\begin{gathered}
\mathbf{s}^{\alpha}=\mathbf{F}^{\mathbf{e}} \cdot \hat{\mathbf{s}}^{\alpha} \\
\mathbf{m}^{\alpha}=\hat{\mathbf{m}}^{\alpha} \cdot\left(\mathbf{F}^{\mathbf{e}}\right)^{-1}
\end{gathered}
$$

where $\mathbf{s}^{\alpha}$ and $\mathbf{m}^{\alpha}$ are the slip direction vector and the slip plane normal vector for slip system $\alpha$ in the current configuration. $\hat{\mathbf{s}}^{\alpha}$ and $\hat{\mathbf{m}}^{\alpha}$ are taken to be orthonormal unit vectors. On the other hand, $\mathbf{s}^{\alpha}$, and $\mathbf{m}^{\alpha}$ are not necessarily unit vectors, but they do remain orthonormal since

$$
\mathbf{m}^{\alpha} \cdot \mathbf{s}^{\alpha}=\hat{\mathbf{m}}^{\alpha} \cdot\left(\mathbf{F}^{\mathbf{e}}\right)^{-1} \cdot \mathbf{F}^{\mathbf{e}} \cdot \hat{\mathbf{s}}^{\alpha}=\hat{\mathbf{m}}^{\alpha} \cdot \hat{\mathbf{s}}^{\alpha}=0
$$

In crystal plasticity models, shearing rates on each slip system $\left(\dot{\gamma}^{\alpha}\right)$ account for plastic deformation via dislocation glide. The evolution of $\mathbf{F}^{\mathbf{P}}$ is defined by the velocity gradient in the intermediate configuration, $\hat{\mathbf{L}}^{\mathbf{P}}$, and depends on $\dot{\gamma}^{\alpha}$ via (Asaro, 1983), i.e., 


$$
\hat{\mathbf{L}}^{\mathbf{P}}=\dot{\mathbf{F}}^{\mathbf{P}} \cdot\left(\mathbf{F}^{\mathbf{P}}\right)^{-1}=\sum_{\alpha=1}^{\mathrm{N}} \dot{\gamma}^{\alpha}\left(\hat{\mathbf{s}}^{\alpha} \otimes \hat{\mathbf{m}}^{\alpha}\right)
$$

The summation in Eq. (3.14) runs over all N slip systems, and $\left(\hat{\mathbf{s}}^{\alpha} \otimes \hat{\mathbf{m}}^{\alpha}\right)$ is the Schmid tensor describing the orientation of the $\alpha$ slip system in the intermediate configuration.

The kinematics based on $\mathbf{F}^{\mathbf{e}}$ and $\mathbf{F}^{\mathbf{P}}$ described up to this point provide the basis for a local crystal plasticity model. Considering compatibility in conjunction with these kinematics provides the framework for a non-local crystal plasticity model. A compatible deformation is defined as a smooth, one-to-one mapping between configurations, i.e., there are no holes or folds (overlapping material) in either the initial or final material bodies. In mathematical terms, a line integral over a path enclosing an area measures compatibility. If the deformation is compatible, there is no closure failure associated with the path. Otherwise, the deformation is considered incompatible.

Within the $\mathbf{F}=\mathbf{F}^{\mathrm{e}} \cdot \mathbf{F}^{\mathbf{P}}$ framework, $\mathbf{F}$ must represent a compatible deformation. The integral equation that represents a compatible deformation can be written as (Steinmann, 1996)

$$
\oint_{c} \mathrm{~d} \mathbf{x}=\oint_{\mathrm{C}} \mathbf{F} \cdot \mathrm{d} \mathbf{X}=0
$$

where $\mathrm{c}$ and $\mathrm{C}$ are closed paths in the current and reference configurations respectively. While $\mathbf{F}^{\mathbf{e}}$ and $\mathbf{F}^{\mathbf{P}}$ taken together must represent a compatible deformation, $\mathbf{F}^{\mathbf{e}}$ and $\mathbf{F}^{\mathbf{P}}$ individually are not necessarily compatible deformations. Except in the special cases when both $\mathbf{F}^{\mathbf{e}}$ and $\mathbf{F}^{\mathbf{P}}$ are compatible (e.g.. uniaxial homogeneous deformation), there will be a net closure failure of a path surrounding an area leading to a non-zero line integral associated with $\mathbf{F}^{\mathrm{e}}$ and $\mathbf{F}^{\mathbf{P}}$. The integral equations associated with the incompatible deformations described by $\mathbf{F}^{\mathbf{e}}$ and $\mathbf{F}^{\mathbf{P}}$ are written as 


$$
\begin{aligned}
& \oint_{\hat{\mathrm{C}}} \mathrm{d} \hat{\mathbf{X}}=\oint_{\mathrm{C}} \mathbf{F}^{\mathrm{e}^{-1}} \cdot \mathrm{d} \mathbf{x} \neq 0 \\
& \oint_{\hat{\mathrm{C}}} \mathrm{d} \hat{\mathbf{X}}=\oint_{\mathrm{C}} \mathbf{F}^{\mathbf{P}} \cdot \mathrm{d} \mathbf{X} \neq 0
\end{aligned}
$$

where $\hat{C}$ is a closed path in the intermediate configuration. The line integrals and net closure failures in Eqs. (3.16) and (3.17) are identical to burgers circuits and burgers vectors used to quantify non-redundant dislocations, otherwise known as GNDs. This connection leads to a link between compatibility and microstructure.

Nye (1953) was one of the first to define a dislocation tensor that quantified GNDs within a continuum. Nye's geometric argument relating the local burgers vector, $\mathbf{b}$, to a general dislocation tensor, $\mathbf{g}$, may be expressed as

$$
\mathbf{b}=\int_{\mathrm{s}} \mathbf{g} \cdot \mathbf{n} \mathrm{da}
$$

where $\mathrm{s}$ is an arbitrary surface and $\mathbf{n}$ is normal to $\mathrm{s}$. In the $\mathbf{F}=\mathbf{F}^{\mathbf{e}} \cdot \mathbf{F}^{\mathbf{P}}$ setting, GNDs are needed to accommodate the incompatibility of the intermediate configuration. Thus, the dislocation tensor representing GNDs should be cast in the intermediate configuration.

An intermediate configuration dislocation tensor, $\hat{\mathbf{G}}$, can be derived from the compatibility equations for $\mathbf{F}^{\mathrm{e}}$ and $\mathbf{F}^{\mathbf{P}}$, Eqs. (3.7) and (3.8), as follows. The line integrals in the compatibility equations can be transformed to surface integrals by Stokes' theorem resulting in (Cermelli and Gurtin, 2001)

$$
\begin{aligned}
& \hat{\mathbf{b}}^{\mathbf{P}}=\oint_{\hat{\mathrm{C}}} \mathrm{d} \hat{\mathbf{X}}=\oint_{\mathrm{C}} \mathbf{F}^{\mathrm{P}} \cdot \mathrm{d} \mathbf{X}=\int_{\mathrm{S}}\left(\operatorname{Curl} \mathbf{F}^{\mathbf{P}}\right)^{\mathrm{T}} \cdot \mathbf{N} \mathrm{dA}=\int_{\hat{\mathrm{S}}} \frac{1}{\mathrm{~J}^{\mathrm{P}}}\left(\operatorname{Curl} \mathbf{F}^{\mathbf{P}}\right)^{\mathrm{T}} \cdot \mathbf{F}^{\mathbf{P}^{\mathrm{T}}} \cdot \hat{\mathbf{N}} \mathrm{d} \hat{\mathrm{A}} \\
& \hat{\mathbf{b}}^{\mathrm{e}}=\oint_{\hat{\mathrm{C}}} \mathrm{d} \hat{\mathbf{X}}=\oint_{\mathrm{c}} \mathbf{F}^{\mathrm{e}^{-1}} \cdot \mathrm{d} \mathbf{x}=\int_{\mathrm{s}}\left(\operatorname{curl} \mathbf{F}^{\mathrm{e}^{-1}}\right)^{\mathrm{T}} \cdot \mathbf{n} \mathrm{da}=\int_{\hat{\mathrm{S}}} \mathrm{J}^{\mathrm{e}}\left(\operatorname{curl} \mathbf{F}^{\mathrm{e}^{-1}}\right)^{\mathrm{T}} \cdot \mathbf{F}^{\mathrm{e}^{-\mathrm{T}}} \cdot \hat{\mathbf{N}} \mathrm{d} \hat{\mathrm{A}}
\end{aligned}
$$

where $\hat{\mathbf{b}}^{\mathbf{P}}$ is the burgers vector in the intermediate configuration associated with $\mathbf{F}^{\mathbf{P}} ; \hat{\mathbf{b}}^{\mathbf{e}}$ is the burgers vector in the intermediate configuration associated with $\mathbf{F}^{\mathrm{e}} ; \mathbf{n}, \mathbf{N}$, and $\hat{\mathbf{N}}$ are 
normal vectors to the surfaces $\mathbf{s}, \mathbf{S}$, and $\hat{\mathbf{S}}$ respectively; $\mathbf{J}^{\mathrm{P}}$ is the determinant of $\mathbf{F}^{\mathbf{P}}$; and $\mathbf{J}^{\mathrm{e}}$ is the determinant of $\mathbf{F}^{\mathrm{e}}$. The final step in Eqs. (3.19) and (3.20) is necessary to move the surface integrals to the intermediate configuration. Comparison of Nye's dislocation equation and the surface integrals in Eqs. (3.19) and (3.20) yield a dislocation tensor associated with $\mathbf{F}^{\mathbf{P}}\left(\hat{\mathbf{G}}^{\mathbf{P}}\right)$ and one associated with $\mathbf{F}^{\mathbf{e}}\left(\hat{\mathbf{G}}^{\mathbf{P}}\right)$

$$
\begin{gathered}
\hat{\mathbf{G}}^{\mathbf{P}}=\frac{1}{\mathrm{~J}^{\mathrm{p}}} \mathbf{F}^{\mathbf{P}} \cdot \operatorname{Curl} \mathbf{F}^{\mathbf{P}} \\
\hat{\mathbf{G}}^{\mathrm{e}}=\mathrm{J}^{\mathrm{e}} \mathbf{F}^{\mathrm{e}^{-1}} \cdot \operatorname{curl} \mathbf{F}^{\mathrm{e}^{-1}} .
\end{gathered}
$$

Cermelli and Gurtin (2001) show that $\hat{\mathbf{b}}^{\mathbf{P}}=\hat{\mathbf{b}}^{\mathrm{e}}$, meaning there is only one dislocation tensor $\hat{\mathbf{G}}$, defined as

$$
\hat{\mathbf{G}}=\frac{1}{\mathbf{J}^{\mathrm{P}}} \mathbf{F}^{\mathrm{P}} \cdot \operatorname{Curl} \mathbf{F}^{\mathbf{P}}=\mathbf{J}^{\mathrm{e}} \mathbf{F}^{\mathbf{e}^{-1}} \cdot \operatorname{curl} \mathbf{F}^{\mathrm{e}^{-1}} .
$$

The dislocation tensor in Eq. (3.23) is one of many that have been proposed in the literature. Examples of other $\mathbf{F}^{\mathbf{e}} \mathbf{F}^{\mathbf{P}}$ based dislocation tensors are

$$
\begin{gathered}
\operatorname{curl}\left(\mathbf{F}^{\mathrm{e}} \cdot \mathbf{F}^{\mathrm{e}^{-\mathrm{T}}}\right) \quad(\text { Bilby et al., 1955) } \\
\operatorname{curl}^{\mathrm{e}}(\text { Acharya and Bassani, 2000) } \\
\operatorname{Curl}^{\mathbf{P}} \text { (Kroner, 1960). }
\end{gathered}
$$

It is also possible within the $\mathbf{F}^{\mathbf{e}} \mathbf{F}^{\mathbf{P}}$ framework to derive a dislocation tensor based on slip gradients. These dislocation tensors are based on an additional compatibility equation (Fleck and Hutchinson, 1997)

$$
\hat{\mathbf{b}}=\oint_{\hat{C}} \hat{\gamma} \mathrm{d} \hat{\mathbf{X}}
$$

where $\hat{\gamma}$ is the slip tensor defined by 


$$
\hat{\gamma}=\sum_{\alpha=1}^{\mathrm{N}} \gamma^{\alpha}\left(\hat{\mathbf{s}}^{\alpha} \otimes \hat{\mathbf{m}}^{\alpha}\right) .
$$

Applying Stokes' theorem to Eq. (3.27) results in a dislocation tensor based on slip gradients with the form

$$
\hat{\mathbf{G}}=\operatorname{curl} \hat{\gamma}
$$

All of the aforementioned dislocation tensors can be applied to materials where GNDs do not affect the initial stress-strain response. At $t=0$, the initial values for the quantities upon which $\hat{\mathbf{G}}$ depends are constant throughout the material, namely $\mathbf{F}_{\mathrm{t}=0}^{\mathbf{P}}=\mathbf{F}_{\mathrm{t}=0}^{\mathbf{e}}=\mathbf{I}$ and $\hat{\boldsymbol{\gamma}}_{\mathrm{t}=0}=\mathbf{0}$. Therefore the initial dislocation tensor, $\hat{\mathbf{G}}_{\mathrm{t}=0}$, equals zero. As plastic deformation accumulates, $\hat{\mathbf{G}}$ evolves resulting in an increased work hardening response. For materials with GNDs that do affect the initial stress-strain response, there is a need to initialize $\hat{\mathbf{G}}$. Determining the initial GND state can be done either by using non-kinematics based methods (for example Evers et al., 2004b) or by augmenting the single crystal kinematics with additional information (for example Polycrystal $\mathbf{F}^{\mathbf{e}} \mathbf{F}^{\mathbf{P}}$ )

\subsubsection{Polycrystal $F^{\mathrm{e}} \mathbf{F}^{\mathrm{P}}$}

Single crystal $\mathbf{F}^{\mathbf{e}} \mathbf{F}^{\mathbf{P}}$ kinematics are routinely applied to polycrystals (e.g. Mathur and Dawson, 1989; Kalidindi et al. 1992). In these models at $\mathrm{t}=0$, the single crystal kinematics take the form shown in Figure 3.2. 


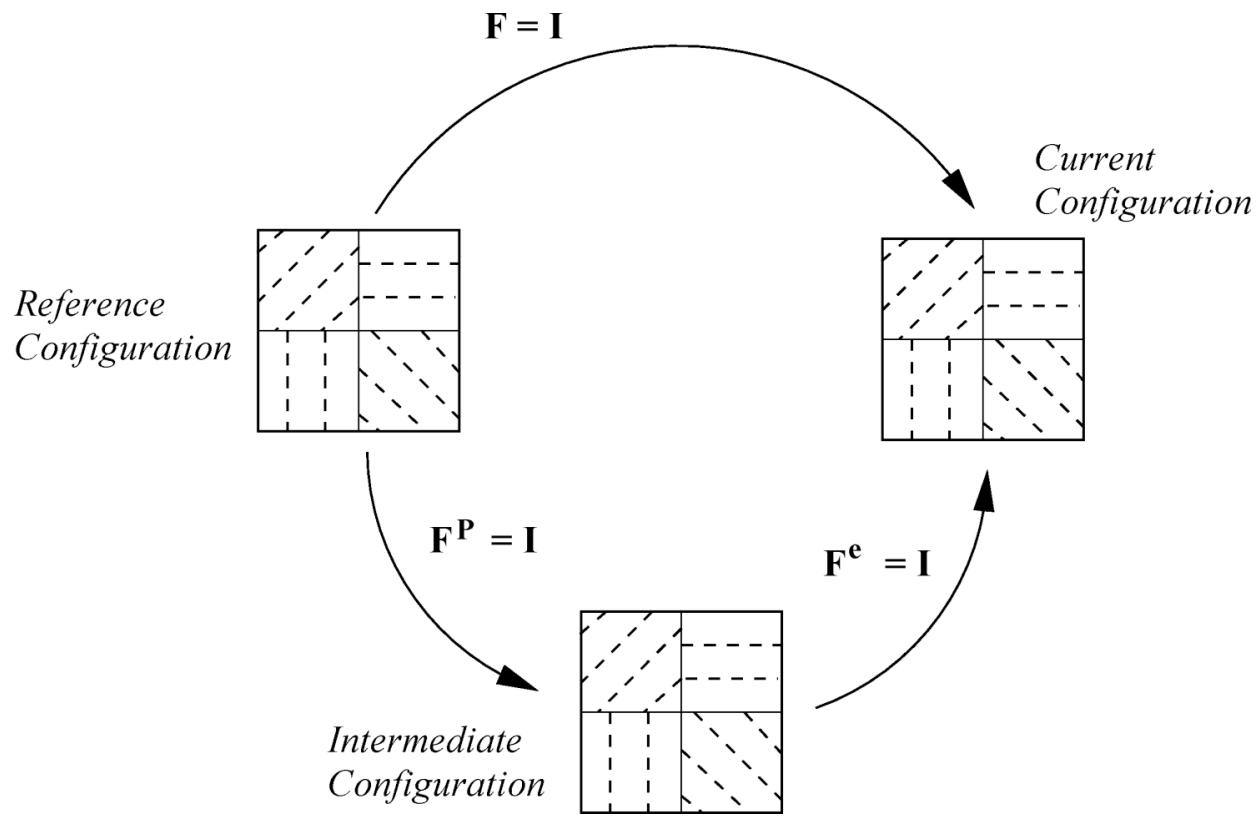

Figure 3.2 - Single crystal kinematics applied to a polycrystal without initialization.

In order to generate a polycrystal in the reference configuration, the initial orientation of each grain ( $\mathbf{R}^{\text {Orient }}$ ) is usually determined via

$$
\mathbf{R}^{\text {Orient }}=\mathbf{R} \cdot \mathbf{R}^{\text {Base }}
$$

where $\mathbf{R}$ is a rotation and $\mathbf{R}^{\text {Base }}$ is an initial state from which all the rotations in the $\mathbf{R}$ field are made. $\mathbf{R}^{\text {Base }}$ can take any orientation value, but it is convenient to make $\mathbf{R}^{\text {Base }}=\mathbf{I}$ making $\mathbf{R}^{\text {Orient }}=\mathbf{R}$. It is important to note that neither $\mathbf{R}$ nor $\mathbf{R}^{\text {Orient }}$ represent a continuous field since each grain in the polycrystal has a different orientation. Because kinematics describes the motions of a material body, rigorously speaking kinematics should include the initialization equation, Eq. (3.30).

When Eq. (3.30) is included in $\mathbf{F}^{\mathbf{e}} \mathbf{F}^{\mathbf{P}}$ kinematics, the kinematics at time $\mathrm{t}=0$ look like Figure 3.3. 


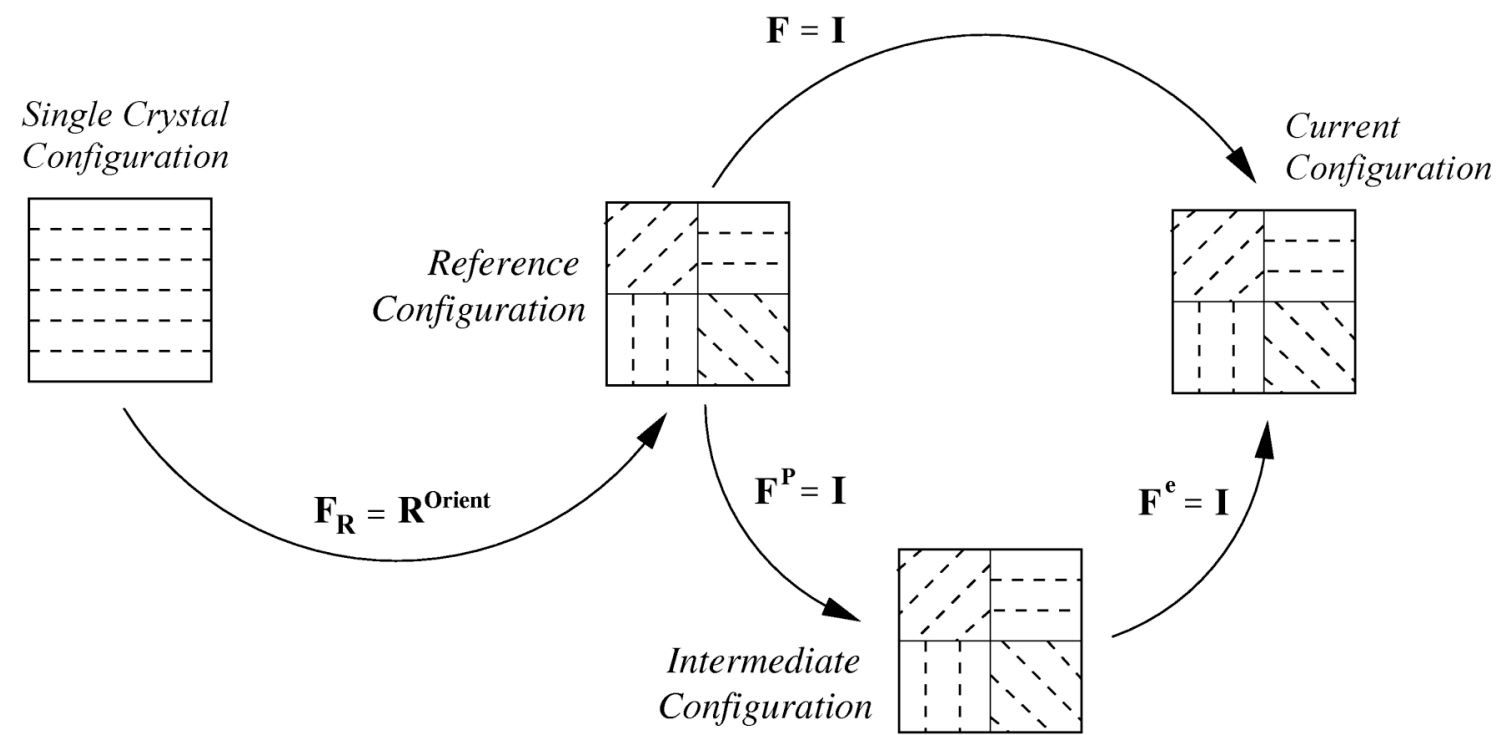

Figure 3.3 - Single crystal kinematics applied to a polycrystal with initialization,

A single crystal configuration has been added to the single crystal kinematics to represent the homogenous single crystal with an orientation $\mathbf{R}^{\text {Base }}$. The term $\mathbf{F}_{\mathbf{R}}$ is introduced so that all mappings between configurations are done via deformation gradients. By the polar decomposition, $\mathbf{F}_{\mathbf{R}}=\mathbf{R}^{\text {Orient }}$ when the left stretch tensor $\left(\mathbf{V}_{\mathbf{R}}\right)$ or the right stretch tensor $\left(\mathbf{U}_{\mathbf{R}}\right)$ equal the identity tensor. By making $\mathbf{U}_{\mathbf{R}}=\mathbf{V}_{\mathbf{R}}=\mathbf{I}$, the reference configuration is stress-free. In this context, $\mathbf{F}_{\mathbf{R}}$ does NOT represent a physical deformation (for that matter neither does $\mathbf{F}^{\mathbf{e}}$ or $\mathbf{F}^{\mathbf{P}}$ ) nor does $\mathbf{F}_{\mathbf{R}}$ account for processing or deformation history. $\mathbf{F}_{\mathbf{R}}$ is strictly a geometric argument needed to produce a polycrystalline material in the reference configuration.

The problem with this approach is that $\mathbf{F}_{\mathbf{R}}$ represents a compatible deformation. Since there is no incompatibility between the single crystal configuration and the reference configuration, there are no GNDs in the polycrystal in the reference configuration. The grain boundaries in an undeformed polycrystal can be described (at least theoretically) by GNDs that accommodate the orientation mismatch between grains. 
In order to provide a kinematics framework that can define a GND state in the reference configuration, the geometric argument in Figure 3.3 has been extended in this work. The grains in the reference configuration polycrystal are no longer described by just a rotation. Rather, the homogeneous material body undergoes a total deformation $\left(\mathbf{F}_{\mathbf{h}}\right)$ to form the polycrystal in the reference configuration as shown in Figure 3.4.

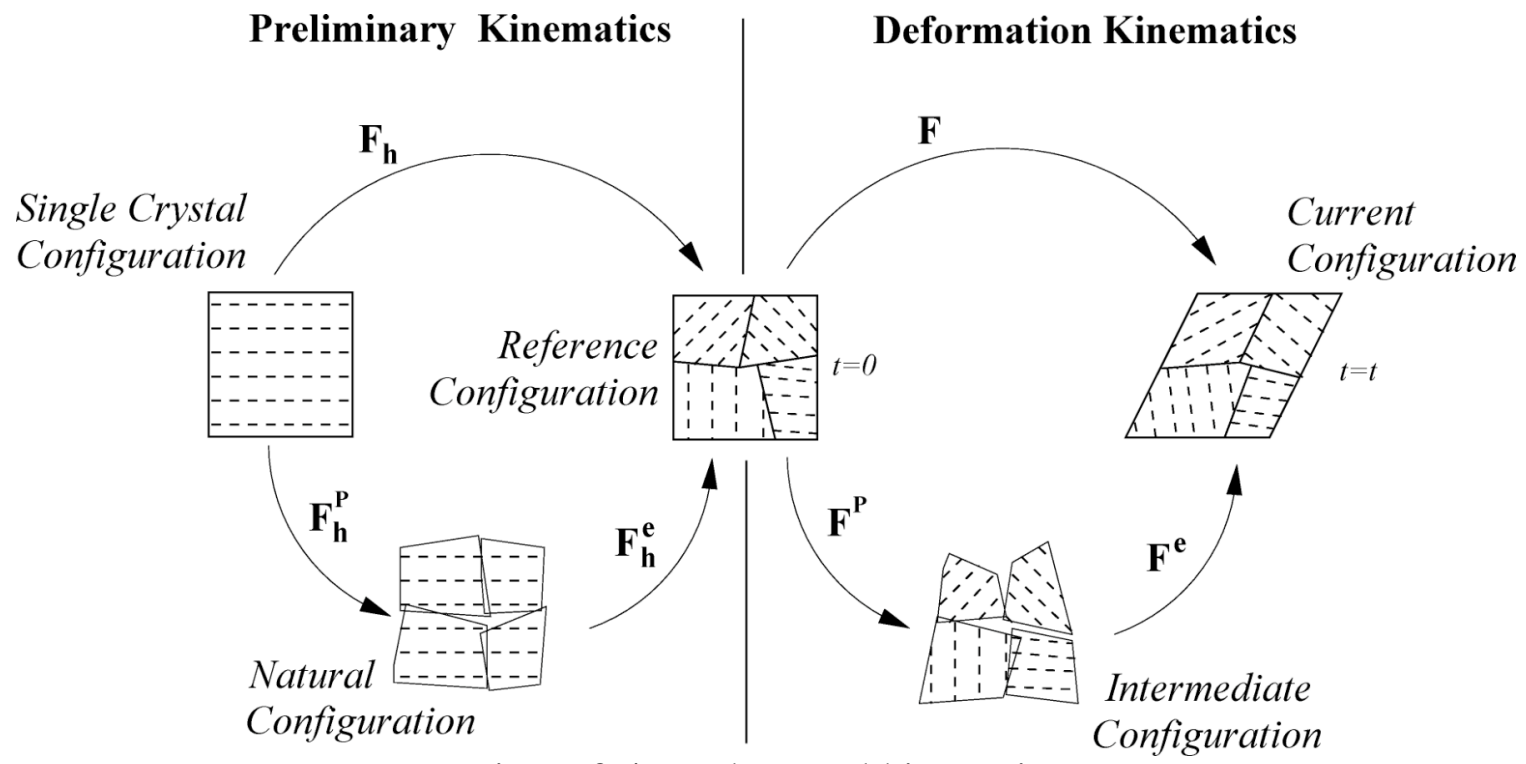

Figure 3.4 - Polycrystal kinematics

The purpose of the preliminary kinematics in Figure 3.4 is to provide a framework by which the initial GND state can be determined in the same manner as the GND state in the intermediate configuration. Note that this kinematic description is a construct, and is in no way intended to describe or represent any actual or prior material processing or other deformation history.

Within the expanded deformation model, $\mathbf{F}_{\mathbf{h}}$ represents an effective deformation (not necessarily a physical deformation) that forms a polycrystal from a single crystal. 
$\mathbf{F}_{\mathbf{h}}$ is multiplicatively decomposed into an elastic part, $\mathbf{F}_{\mathbf{h}}^{\mathbf{e}}$, and plastic part, $\mathbf{F}_{\mathbf{h}}^{\mathbf{P}}$, as follows

$$
\mathbf{F}^{\mathrm{h}}=\mathbf{F}_{\mathrm{h}}^{\mathrm{e}} \cdot \mathbf{F}_{\mathrm{h}}^{\mathrm{P}} .
$$

By separating $\mathbf{F}_{\mathrm{h}}$ in a similar fashion as $\mathbf{F}$, many of the same ideas and concepts associated with the single crystal kinematics are applied with the preliminary kinematics.

Just as before, the single crystal is initially deformed by $\mathbf{F}_{\mathbf{h}}^{\mathbf{P}}$, producing shape changes within the material but no orientation changes. The resulting shape changes create incompatibility in the form of voids and overlaps in the natural configuration as shown in Figure 3.5.

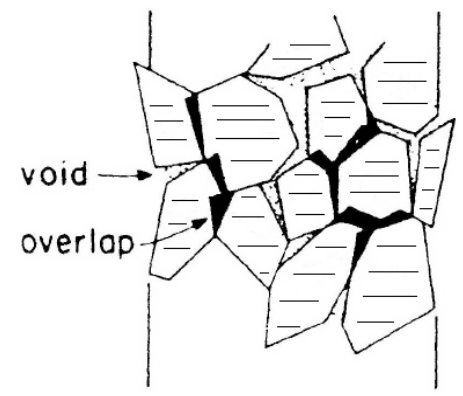

Figure 3.5 - An incompatible crystal in the natural configuration

The lattice planes are then elastically stretched and rotated by $\mathbf{F}_{\mathbf{h}}^{\mathbf{e}}$ resulting in a compatible polycrystal in the reference configuration. The stretching and rotation of the lattice planes described by $\mathbf{F}_{h}^{\mathrm{e}}$ can be decoupled since $\mathbf{F}_{h}^{\mathrm{e}}=\mathbf{R}_{\mathrm{h}}^{\mathrm{e}} \mathbf{U}_{\mathrm{h}}^{\mathrm{e}}$, where $\mathbf{U}_{\mathrm{h}}^{\mathrm{e}}$ represents the stretching and $\mathbf{R}_{\mathrm{h}}^{\mathrm{e}}$ the rotation.

It is important to understand the role of $\mathbf{F}_{h}^{\mathbf{P}}, \mathbf{U}_{\mathrm{h}}^{\mathrm{e}}$, and $\mathbf{R}_{\mathrm{h}}^{\mathrm{e}}$ within this augmented kinematic description. $\mathbf{F}_{\mathbf{h}}^{\mathbf{P}}$ creates the incompatibility in the natural configuration. 
Without $\mathbf{F}_{\mathbf{h}}^{\mathbf{P}}$, the natural configuration does not exist and $\mathbf{F}_{\mathbf{h}}$ represents a compatible deformation. $\mathbf{U}_{\mathbf{h}}^{\mathrm{e}}$ describes the internal stress-state of the reference configuration polycrystal, and $\mathbf{R}_{\mathrm{h}}^{\mathrm{e}}$ characterizes the orientation state of the reference configuration polycrystal. With this in mind, the $\mathbf{F}_{\mathbf{h}}^{\mathbf{e}} \mathbf{F}_{\mathbf{h}}^{\mathbf{P}}$ based kinematics can describe a stress-free, GND-free polycrystal in the reference configuration (i.e. the polycrystal created by $\mathbf{F}_{\mathbf{h}}=\mathbf{R}^{\text {Orient }}$ in Figure 3.3) by assuming $\mathbf{F}_{\mathbf{h}}^{\mathbf{P}}=\mathbf{I}, \mathbf{U}_{\mathbf{h}}^{\mathrm{e}}=\mathbf{I}$, and $\mathbf{R}_{\mathrm{h}}^{\mathrm{e}}=\mathbf{R}^{\text {Orient }}$.

By applying the $\mathbf{F}^{\mathbf{e}} \mathbf{F}^{\mathbf{P}}$ dislocation tensor concepts derived by Cermelli and Gurtin (2001) to $\mathbf{F}_{\mathbf{h}}^{\mathbf{e}} \mathbf{F}_{\mathbf{h}}^{\mathbf{P}}$, a dislocation tensor in the natural configuration $(\tilde{\mathbf{G}})$ is defined as

$$
\tilde{\mathbf{G}}=\frac{1}{\mathbf{J}^{\mathbf{F}}} \mathbf{F}_{\mathbf{h}}^{\mathbf{P}} \operatorname{Curl} \mathbf{F}_{\mathbf{h}}^{\mathbf{P}}=\mathbf{J}^{\mathbf{F}_{\mathbf{h}}^{e}} \mathbf{F}_{\mathbf{h}}^{\mathbf{e}^{-1}} \operatorname{curl} \mathbf{F}_{\mathbf{h}}^{\mathbf{e}^{-1}}
$$

where $\mathbf{J}^{\mathbf{F}_{h}^{p}}$ is the determinant of $\mathbf{F}_{h}^{\mathbf{P}}$ and $\mathbf{J}^{\mathbf{F}_{h}^{p}}$ is the determinant of $\mathbf{F}_{h}^{\mathbf{e}}$. Physically, $\mathbf{G}$ represents the GND state needed for compatibility in the reference configuration. Two examples of GND structures that $\mathbf{G}$ could represent are high angle grain boundaries, and low angle tilt boundaries.

In this work, the polycrystal in the reference configuration is assumed to be stressfree because very little is known about the initial internal stress state. With this assumption $^{4}, \mathbf{U}_{\mathbf{h}}^{\mathrm{e}}=\mathbf{I}$, and $\mathbf{F}_{\mathrm{h}}^{\mathrm{e}}=\mathbf{R}_{\mathrm{h}}^{\mathrm{e}}$ and the initial GND state of the polycrystal can be determined by

$$
\tilde{\mathbf{G}}=\mathbf{R}_{\mathrm{h}}^{\mathbf{e}^{-1}} \operatorname{Curl} \mathbf{R}_{\mathrm{h}}^{\mathbf{e}^{-1}}
$$

\footnotetext{
${ }^{4}$ It is not necessary within the $\mathbf{F}_{\mathbf{h}} \mathbf{F}_{\mathbf{h}}^{\mathbf{P}}$ kinematics to make the polycrystal in the reference configuration stress-free. In this case, $\tilde{\mathbf{G}}$ would be a function of $\mathbf{F}_{\mathrm{h}}^{\mathbf{e}}$ and not just $\mathbf{R}_{\mathrm{h}}^{\mathbf{e}}$.
} 
where $\mathbf{J}^{\mathbf{R}_{\mathbf{e}}^{\mathbf{h}}}=1$. Within this context, the values of $\mathbf{F}_{\mathbf{h}}$ and $\mathbf{F}_{\mathbf{h}}^{\mathbf{P}}$ are not important because all the information needed to determine the initial GND state is contained within $\mathbf{F}_{\mathbf{h}}^{\mathbf{e}}$ and in particular $\mathbf{R}_{\mathbf{h}}^{\mathrm{e}}$.

$\tilde{\mathbf{G}}$ is then pushed forward to the intermediate configuration using $\mathbf{R}_{\mathbf{h}}^{\mathbf{e}}$ (to move from the natural to the reference configuration) and $\mathbf{F}^{\mathbf{P}}$ (to move from the reference to the intermediate configuration). The total GND tensor in the intermediate configuration, $\hat{\mathbf{G}}^{\text {Tot }}$, is defined as the sum of $\tilde{\mathbf{G}}$, moved from the natural configuration to the intermediate configuration, and $\hat{\mathbf{G}}$ :

$$
\hat{\mathbf{G}}^{\mathrm{Tot}}=\mathbf{F}^{\mathbf{p}} \cdot \mathbf{R}^{\mathrm{h}-\mathrm{e}} \cdot \tilde{\mathbf{G}} \cdot \mathbf{R}^{\mathrm{h}-\mathrm{e}^{\mathrm{T}}} \cdot \mathbf{F}^{\mathbf{p}^{-1}}+\hat{\mathbf{G}}
$$

where $\hat{\mathbf{G}}$ is defined in Eq. 3.22.

With this formulation, all misorientations within a material are accommodated by GNDs. Such a description is certainly applicable to a low angle boundary (a boundary with a misorientation less than $10^{\circ}-15^{\circ}$ ). However, describing a high angle boundary as a dislocation array of GNDs may not be ideal. In addition, special boundaries (a high angle boundary with a low grain boundary energy) are not considered with this formulation.

Despite these shortcomings, the kinematics approach that defines $\hat{\mathbf{G}}^{\text {Tot }}$ is an important first step towards describing grain boundaries and other dislocation arrays that exist in a material body in the reference configuration. 


\subsection{Thermodynamics}

When developing a crystal plasticity model with internal state variables (ISVs), it is important to ensure that the model does not violate the $1^{\text {st }}$ or $2^{\text {nd }}$ law of thermodynamics. The thermodynamics of ISV models was developed by Coleman and Noll (1963) and Coleman and Gurtin (1967), who applied the basic principles of Gibbsian thermodynamics to ISV models. In this section, the approach developed by Coleman-Noll is applied to local, gradient, and non-local integral ISV models.

\subsubsection{Local ISV Model}

Take a material body B in the current configuration upon which only the Cauchy stress $(\boldsymbol{\sigma})$ does work during deformation. The $1^{\text {st }}$ law of thermodynamics for this material body is formulated with integrals as

$$
\int_{B} \rho_{\text {Den }} \dot{E} d V=\int_{B} \boldsymbol{\sigma}: \mathbf{L} d V-\oint_{\partial B} \mathbf{q} \cdot \mathbf{n} d a+\int_{B} \rho_{\text {Den }} h d V
$$

where $\rho$ is the mass density, $\mathrm{E}$ is the internal energy per unit mass, $\mathbf{L}$ the velocity gradient, $\mathbf{q}$ represents the heat flux vector, $\mathbf{n}$ is a normal to the surface $\partial \mathrm{B}$, and $\mathrm{h}$ represents entropy flux. After using the divergence theorem to change the surface integral to a volume integral, the local, point-wise form of the $1^{\text {st }}$ law can be written as

$$
\rho_{\text {Den }} \dot{\mathrm{E}}=\boldsymbol{\sigma}: \mathbf{L}-\nabla \cdot \mathbf{q}+\rho_{\text {Den }} \mathrm{h} .
$$

The conversion from the integral form to the local, point-wise form is mathematically possible because when each point in the material body B satisfies Eq. (3.36), then the integral form of the $1^{\text {st }}$ law over the entire body B must hold. 
The $2^{\text {nd }}$ law of thermodynamics is also an integral equation

$$
\int_{B} \rho_{\text {Den }} \dot{S} d V+\oint_{\partial в} \frac{\mathbf{q}}{T} \cdot \mathbf{n} d a-\int_{B} \rho_{\text {Den }} \frac{h}{T} d V \geq 0
$$

where $\mathrm{S}$ is entropy and $\mathrm{T}$ is temperature. After applying the divergence theorem to the surface integral, the local, point-wise form of the $2^{\text {nd }}$ law becomes

$$
\rho_{\text {Den }} \dot{\mathrm{S}}+\nabla \cdot\left(\frac{\mathbf{q}}{\mathrm{T}}\right)-\rho_{\text {Den }} \frac{\mathrm{h}}{\mathrm{T}} \geq 0
$$

The final thermodynamic quantity that the Coleman-Noll approach uses is the Helmholtz free energy per unit mass $(\psi)$, which is defined as

$$
\psi=\mathrm{E}-\mathrm{ST}
$$

Combining Eqs. (3.39), (3.36), and (3.38) leads to a combined $1^{\text {st }}$ and $2^{\text {nd }}$ law

$$
-\rho_{\text {Den }}(\dot{\psi}+\mathrm{ST})+\boldsymbol{\sigma}: \mathbf{L}+\frac{\mathbf{q} \cdot \nabla \mathrm{T}}{\mathrm{T}} \geq 0
$$

Equation (3.40) provides the basis for the Coleman-Noll approach.

The first step in the Coleman-Noll approach involves proposing a constitutive equation for $\psi$. Equation (3.39) is the general form for $\psi$ and applies to all materials. The proposed constitutive equation for $\psi$ is specific to the material and includes the variables of interest. When modeling metals with a local ISV model, it is often assumed that $\psi$ depends only on elastic strain $\left(\boldsymbol{\varepsilon}^{\mathbf{e}}\right)$ and T

$$
\psi=\bar{\psi}\left(\varepsilon^{\mathrm{e}}, \mathrm{T}\right)
$$

Via the chain rule on Eq. (3.41), $\psi$ can be expressed as

$$
\dot{\psi}=\frac{\partial \psi}{\partial \varepsilon^{\mathrm{e}}} \dot{\boldsymbol{\varepsilon}}^{\mathrm{e}}+\frac{\partial \psi}{\partial \mathrm{T}} \dot{\mathrm{T}} .
$$

Combining Eq. (3.40) with Eq. (3.42) and grouping similar terms results in 


$$
\left(\boldsymbol{\sigma}-\rho_{\text {Den }} \frac{\partial \psi}{\partial \dot{\boldsymbol{\varepsilon}}^{\mathbf{e}}}\right) \dot{\boldsymbol{\varepsilon}}^{\mathrm{e}}-\rho_{\text {Den }}\left(\mathrm{S}+\frac{\partial \psi}{\partial \mathrm{T}}\right) \dot{\mathrm{T}}+\boldsymbol{\sigma}: \dot{\boldsymbol{\varepsilon}}^{\mathbf{P}}-\frac{1}{\mathrm{~T}} \mathbf{q} \cdot \nabla \mathrm{T} \geq 0 .
$$

A small elastic strain assumption $\left(\dot{\boldsymbol{\varepsilon}}^{\mathrm{e}} \approx \mathbf{L}^{\mathrm{E}}\right)$ is embedded in Eq. (3.43). Since $\dot{\boldsymbol{\varepsilon}}^{\mathrm{e}}$ and $\dot{\mathrm{T}}$ can be arbitrarily assigned, the only way to guarantee that the inequality in Eq. (3.43) holds is if

$$
\sigma=\rho_{\text {Den }} \frac{\partial \psi}{\partial \varepsilon^{\mathrm{e}}} \text { and } S=-\frac{\partial \psi}{\partial \mathrm{T}} .
$$

Equation (3.44) represents an important result of the Coleman-Noll thermodynamic analysis. All the stresses that do work on the material (in this case only $\sigma$ ) and the entropy are defined in terms of $\psi$. Physically Eq. (3.44) can be interpreted as the mechanical work done by $\sigma$ on the material body increases the internal energy of the body as a function of $\boldsymbol{\varepsilon}^{\mathrm{e}}$.

The other important result of the thermodynamic analysis is the dissipation inequality

$$
\boldsymbol{\sigma}: \dot{\boldsymbol{\varepsilon}}^{\mathbf{P}}-\frac{1}{\mathrm{~T}} \mathbf{q} \cdot \nabla \mathrm{T} \geq 0 .
$$

In the dissipation inequality any and all mechanisms by which the material body dissipates energy are accounted for. Specifically, Eq. (3.45) states that some of the mechanical work, $\sigma: \dot{\boldsymbol{\varepsilon}}^{\mathrm{P}}$, irreversibly becomes heat and is dissipated from the material body. If no energy was dissipated, the deformation would be reversible, which would make it an elastic deformation. Hence, the dissipation potential is essential to all ISV plasticity models.

In short, thermodynamics provides a framework from which to build an ISV model. All the stresses that do work and all the dissipation mechanisms are identified 
and defined. Arbitrarily adding or neglecting any of the defined stresses or dissipation mechanisms violates the $1^{\text {st }}$ and $2^{\text {nd }}$ laws of thermodynamics. How the model is built within this framework is left to the modeler. Note that there are no thermodynamic restrictions on the constitutive form of $\psi$. For example, if $\psi$ is assumed to be quadratic in elastic strain like

$$
\rho_{\text {Den }} \psi=\bar{\psi}\left(\boldsymbol{\varepsilon}^{\mathrm{e}}, \mathrm{T}\right)=\frac{1}{2} \boldsymbol{\varepsilon}^{\mathrm{e}}: \underset{\sim}{\boldsymbol{L}}: \boldsymbol{\varepsilon}^{\mathrm{e}}+\mathrm{g}(\mathrm{T})
$$

where $\mathrm{g}(\mathrm{T})$ represents the temperature dependence, then Eq. (3.46) results in standard linear elasticity

$$
\boldsymbol{\sigma}=\rho_{\text {Den }} \frac{\partial \psi}{\partial \varepsilon^{\mathrm{e}}}=\underset{\sim}{\boldsymbol{L}}: \boldsymbol{\varepsilon}^{\mathrm{e}}
$$

\subsubsection{Gradient ISV Models}

The thermodynamic analysis of gradient ISV models is built on the local, pointwise $1^{\text {st }}$ and $2^{\text {nd }}$ laws even though these models contain "non-local" like gradient variables. When the $1^{\text {st }}$ and $2^{\text {nd }}$ laws are localized, all the information in the region surrounding the point is lost. Without this information, the gradient quantity cannot be calculated. Therefore, all gradient quantities are independent variables and as such they must be included in the constitutive equation for $\psi$.

In gradient ISV models, there are two types of gradient variables: (1) gradient variables associated with higher order stresses and (2) gradient variables associated with dissipation. Because these two gradient variables represent different physics, these variables are handled differently in the thermodynamic analysis. The Coleman-Noll 
approach to both types of gradient variables is illustrated in following example of a gradient ISV model that is based on the work of Dillon and Krachtvil (1970) and Regueiro et al. (2002).

In the work of Dillon and Krachtvil (1970), they assumed that higher order stresses did work on the material, and further that these higher order stresses were related to microstructure. This assumption led to a local, point-wise first law

$$
\rho_{\text {Den }} \dot{\mathrm{E}}=\sigma: \mathbf{L}+\mathbf{z}_{3}: \nabla \mathbf{L}-\nabla \cdot \mathbf{q}+\rho_{\text {Den }} \mathbf{h},
$$

where $\underset{3}{\mathbf{v}}$ is a third order couple stress conjugate to gradient of the velocity gradient ( $\nabla \mathbf{L})$. Following the work of Regueiro et al. (2002), microstructure is also assumed to dissipate energy. This dissipation is captured with a gradient variable $\boldsymbol{\Upsilon}$, which is a dislocation tensor (Eq. 3.23). It is further assumed that $\psi$ explicitly contains $\mathbf{\Upsilon}$, i.e.

$$
\frac{\partial \psi}{\partial \Upsilon} \neq 0
$$

Thus, the constitutive equation for $\psi$ includes $\boldsymbol{\varepsilon}^{\mathrm{e}}$ and $\mathrm{T}$ as well as the two gradient terms $\nabla \boldsymbol{\varepsilon}^{\mathrm{e}}$ (assuming $\mathbf{L} \approx \dot{\boldsymbol{\varepsilon}}^{\mathrm{e}}+\dot{\boldsymbol{\varepsilon}}^{\mathrm{P}}$ ) and $\Upsilon$

$$
\psi=\bar{\psi}\left(\varepsilon^{\mathrm{e}}, \nabla \boldsymbol{\varepsilon}^{\mathrm{e}}, \Upsilon, \mathrm{T}\right)
$$

Carrying out the Coleman-Noll analysis results in

$$
\begin{aligned}
& \left(\boldsymbol{\sigma}-\rho_{\text {Den }} \frac{\partial \psi}{\partial \boldsymbol{\varepsilon}^{\mathbf{e}}}\right) \dot{\boldsymbol{\varepsilon}}^{\mathrm{e}}+\left(\mathbf{v}-\rho_{\text {Den }} \frac{\partial \psi}{\partial\left(\nabla \boldsymbol{\varepsilon}^{\mathbf{e}}\right)}\right) \nabla \dot{\boldsymbol{\varepsilon}}^{\mathrm{e}}-\rho_{\text {Den }}\left(\mathrm{S}+\frac{\partial \psi}{\partial \mathrm{T}}\right) \dot{\mathrm{T}}+ \\
& \boldsymbol{\sigma}: \dot{\boldsymbol{\varepsilon}}^{\mathbf{P}}+\underset{3}{\mathbf{v}}: \nabla \dot{\boldsymbol{\varepsilon}}^{\mathbf{P}}-\frac{\partial \psi}{\partial \Upsilon} \dot{\boldsymbol{\Upsilon}}-\frac{1}{\mathrm{~T}} \mathbf{q} \cdot \nabla \mathrm{T} \geq 0
\end{aligned}
$$

Since the rates in Eq. (3.51) can be arbitrarily assigned, the only way to guarantee that the inequality in Eq. (3.51) holds is if 


$$
\boldsymbol{\sigma}=\rho_{\text {Den }} \frac{\partial \psi}{\partial \boldsymbol{\varepsilon}^{\mathrm{e}}}, \underset{3}{\mathbf{v}}=\rho_{\text {Den }} \frac{\partial \psi}{\partial\left(\nabla \boldsymbol{\varepsilon}^{\mathrm{e}}\right)} \text {, and } \mathrm{S}=-\frac{\partial \psi}{\partial \mathrm{T}}
$$

In this model, there are two stresses defined in terms of $\psi: \sigma$ and $\underset{3}{\mathbf{v}}$. Thus, mechanical work done by $\boldsymbol{\sigma}$ and $\underset{3}{\mathbf{v}}$ increases the internal energy of the material body as a function of $\boldsymbol{\varepsilon}^{\mathbf{e}}$ and $\nabla \boldsymbol{\varepsilon}^{\mathbf{e}}$. In addition, the dissipation inequality is

$$
\boldsymbol{\sigma}: \dot{\boldsymbol{\varepsilon}}^{\mathbf{P}}+\underset{3}{\mathbf{v}}: \nabla \dot{\boldsymbol{\varepsilon}}^{\mathbf{P}}-\frac{\partial \psi}{\partial \boldsymbol{\Upsilon}} \dot{\boldsymbol{\Upsilon}}-\frac{1}{\mathrm{~T}} \mathbf{q} \cdot \nabla \mathrm{T} \geq 0
$$

In Eq. (3.53), $\frac{\partial \psi}{\partial \Upsilon}$ is an internal force within the material that dissipates energy (much like a friction force). This internal force is not included in the $1^{\text {st }}$ law because work done by internal forces results in energy changing forms not a change in the total energy of the material body. In this case, mechanical energy $\left(\boldsymbol{\sigma}: \dot{\boldsymbol{\varepsilon}}^{\mathbf{P}}\right.$ and $\left.\underset{3}{\mathbf{v}}: \nabla \dot{\boldsymbol{\varepsilon}}^{\mathbf{P}}\right)$ is irreversibly stored by the microstructure $\left(\frac{\partial \psi}{\partial \Upsilon}\right)$ or transformed to heat and heat $\left(\frac{1}{\mathrm{~T}} \mathbf{q} \cdot \nabla \mathrm{T}\right)$.

There is no consensus in the literature as to the best way to represent microstructure with gradient variables. Most gradient models represent the effects of microstructure with gradient variables associated with higher order stresses or with dissipation, but not both. The advantage of models based on gradient variables associated with higher order stresses is that they can be length scale sensitive in both the elastic and plastic regimes since the higher order stresses can be present in both regimes. The disadvantage of these models is that the application of boundary conditions and equilibrium to higher order stresses is usually complicated.

Implementing gradient models with gradient dissipation type gradient variables is usually easier since there are no higher order stresses that do work on the material. 
Therefore, boundary conditions and equilibrium are applied only to $\sigma$ in the conventional manner. If there are any internal forces defined over a volume (as opposed to those defined in a stastitistical manner) in the material, they should be equilibrated. Applying boundary conditions and equilibrium to $\sigma$ only implies that all the internal forces defined over a volume are self-equilibrating. The other disadvantage to this approach is that gradient variables representing dissipation only provide a length scale in the plastic regime because no energy is dissipated in the elastic regime.

\subsubsection{ISV Models with Non-Local Integral Variables}

Eringen (1981) developed the general thermodynamic formulation for non-local integral plasticity models. In Eringen's approach, the local form of the $1^{\text {st }}$ and $2^{\text {nd }}$ law is augmented with non-local residual terms. These non-local residuals are introduced into the $1^{\text {st }}$ and $2^{\text {nd }}$ laws to account for energy loss or gain (in the $1^{\text {st }}$ law) and local entropy flux (in the $2^{\text {nd }}$ law) between the material point and the rest of the material body.

In this example, there are no non-local residual terms in the $1^{\text {st }}$ or $2^{\text {nd }}$ law. Rather, three non-local integral variables are introduced into the ISV model. The first non-local variable is a function of $\boldsymbol{\varepsilon}^{\mathbf{e}}$

$$
\left\{\boldsymbol{\varepsilon}^{\mathrm{e}}\right\}=\int_{V}\left[\boldsymbol{\varepsilon}^{\mathrm{e}}(\mathbf{x})-\boldsymbol{\varepsilon}^{\mathrm{e}}(\boldsymbol{\xi})\right] \operatorname{Exp}(-\mathrm{r}) \mathrm{d} \mathbf{V}
$$

the second is a function of $\gamma$

$$
\{\gamma\}=\int_{V}[\gamma(\mathbf{x})-\gamma(\xi)] \operatorname{Exp}(-r) d \mathbf{V}
$$

and the third is a function of $\mathbf{R}$ 


$$
\{\mathbf{R}\}=\int_{V}[\mathbf{R}(\mathbf{x})-\mathbf{R}(\xi)] \operatorname{Exp}(-\mathrm{r}) \mathrm{d} \mathbf{V}
$$

The variable $\mathbf{R}$ is different than $\boldsymbol{\varepsilon}^{\mathbf{e}}$ and $\gamma$ in that $\mathbf{R}$ has an initial value that does not change.

The constitutive equation for $\psi$ depends only on $\boldsymbol{\varepsilon}^{\mathbf{e}},\{\gamma\}, \mathbf{R}$, and $\mathrm{T}$

$$
\psi=\bar{\psi}\left(\varepsilon^{\mathrm{e}},\{\gamma\}, \mathbf{R}, \mathrm{T}\right)
$$

$\left\{\boldsymbol{\varepsilon}^{\mathrm{e}}\right\}$ does not appear in $\bar{\psi}$ because $\left\{\boldsymbol{\varepsilon}^{\mathrm{e}}\right\}$ is defined in terms of the local variable $\boldsymbol{\varepsilon}^{\mathrm{e}}$,

which is already included in $\bar{\psi} \cdot\{\gamma\}$ does appear in $\bar{\psi}$ because the local variable $\gamma$ does not appear in $\bar{\psi}$. Carrying out the Coleman-Noll analysis results in

$$
\left(\boldsymbol{\sigma}-\rho_{\text {Den }} \frac{\partial \psi}{\partial \boldsymbol{\varepsilon}^{\mathrm{e}}}\right) \dot{\boldsymbol{\varepsilon}}^{\mathrm{e}}-\rho_{\text {Den }}\left(\mathrm{S}+\frac{\partial \psi}{\partial \mathrm{T}}\right) \dot{\mathrm{T}}+\boldsymbol{\sigma}: \dot{\boldsymbol{\varepsilon}}^{\mathbf{P}}-\frac{\partial \psi}{\partial\{\gamma\}}\{\dot{\gamma}\}-\frac{\partial \psi}{\partial\{\mathbf{R}\}} \dot{\mathbf{R}}-\frac{1}{\mathrm{~T}} \mathbf{q} \cdot \nabla \mathrm{T} \geq 0 .
$$

A small elastic strain assumption ( $\dot{\boldsymbol{\varepsilon}}^{\mathrm{e}} \approx \mathbf{L}^{\mathrm{E}}$ ) is embedded in Eq. (3.58). The term $\dot{\mathbf{R}}=\mathbf{0}$ because $\mathbf{R}$ is constant meaning that $\mathbf{R}$ does not need to appear in $\bar{\psi}$. Since $\dot{\boldsymbol{\varepsilon}}^{\mathrm{e}}$ and $\dot{\mathbf{S}}$ can be arbitrarily assigned, the only way to guarantee that the inequality in Eq. (3.58) holds is if

$$
\sigma=\rho_{\text {Den }} \frac{\partial \psi}{\partial \varepsilon^{\mathrm{e}}}, \text { and } S=-\frac{\partial \psi}{\partial \mathrm{T}}
$$

The dissipation inequality is then

$$
\boldsymbol{\sigma}: \dot{\boldsymbol{\varepsilon}}^{\mathrm{e}}-\frac{\partial \psi}{\partial\{\gamma\}}\{\dot{\gamma}\}-\frac{1}{\mathrm{~T}} \mathbf{q} \cdot \nabla \mathrm{T} \geq 0
$$

In Eq. (3.60) $\frac{\partial \psi}{\partial\{\gamma\}}$ represents an internal force that dissipates energy; no different than the internal force $\frac{\partial \psi}{\partial \Upsilon}$ in the gradient ISV example. 
The advantage of using non-local integral variables can be seen in the treatment

of $\left\{\boldsymbol{\varepsilon}^{\mathrm{e}}\right\}$. If $\left\{\boldsymbol{\varepsilon}^{\mathrm{e}}\right\}$ was the only non-local variable, the resulting thermodynamic analysis would be exactly the same as the local ISV example presented earlier. Thus it is possible to construct an ISV model based on non-local integral variables in which there are neither higher order stresses nor any unequilibrated internal forces in the dissipation inequality. In this case boundary conditions and equilibrium are applied only to $\sigma$ and there is no assumption that all other forces are self-equilibrating.

\subsection{Kinetics}

The kinetics of plastic deformation in a crystal plasticity model account for largescale dislocation motion and their collective effect on the mechanical response. The basic premise for these relationships is that large-scale dislocation motion occurs when the resolved shear stress on a slip system $\left(\tau^{\alpha}\right)$ reaches a critical value $\left(\tau^{\mathrm{CSS}-\alpha}\right)$. As dislocations move along a slip system, they inhibit the motion of other dislocations, resulting in an increase in $\tau^{\text {CSS- } \alpha}$. This section includes a brief overview of how the kinetics of dislocation motion and work hardening are implemented in crystal plasticity.

\subsubsection{Dislocation Motion}

Since crystal plasticity models do not account for individual dislocations, the kinetics of large-scale dislocation motion in crystal plasticity models are represented by the rate of slip on each slip system $\alpha\left(\dot{\gamma}^{\alpha}\right)$. There are two different numerical approaches 
employed to solve for $\dot{\gamma}^{\alpha}$ : rate-independent formulations and rate-dependent

formulations. In the rate-independent approach the determination of the slip rates is an inverse problem, while in the rate dependent approach the slip rates are calculated directly.

\section{$\underline{\text { 3.3.1.1 Rate-Independent Formulations }}$}

In the rate-independent approach, the Jaumann corotational derivative and the slip plane hardening rates form the basis for a system of equations in which $\dot{\gamma}^{\alpha}$ is a function of either the stress rate or the deformation rate. This system of equations is then inverted in order to determine $\dot{\gamma}^{\alpha}$. A number of different numerical approaches have been developed to solve rate-independent boundary value problems. For details on these solution schemes see Peirce et al., 1982; Anand and Kothari, 1996; and Schroder and Miehe, 1997; McGinty and McDowell, 2006.

Major shortcomings of rate-independent formulations are determining which slip systems are active and the lack of a unique solution in many problems. If five slip systems are not active, the selection of slip systems required to produce an arbitrary deformation is not necessarily unique.

\subsubsection{Rate-Dependent Formulations}

In rate-dependent formulations, an equation for the slip rates on each slip system is specified. This equation, or flow rule, specifies that $\dot{\gamma}^{\alpha}$ is a function of $\tau^{\alpha}$ and $\tau^{\mathrm{CSS}-\alpha}$. 
Commonly flow rules are either a power law expression or a dislocation velocity based equation. The power law flow rule in its most basic form applies to materials deformed via quasi-static strain rates under isothermal conditions and has the form (Hutchinson, 1976)

$$
\dot{\gamma}^{\alpha}=\dot{\gamma}_{0}\left|\frac{\tau^{\alpha}}{\tau^{\mathrm{CSS}-\alpha}}\right|^{\frac{1}{\mathrm{~m}}} \operatorname{sgn}\left(\tau^{\alpha}\right)
$$

where $\dot{\gamma}_{0}$ is the reference shear strain rate, and $\mathrm{m}$ is a rate sensitivity parameter. Equation (3.61) has been modified to include other factors like temperature and backstress (Meric et al., 1994; Harder, 1999; Bamman, 2001).

Dislocation velocity based flow rules make use of the Orowan equation

$$
\dot{\gamma}^{\alpha}=\rho^{\alpha} b v^{\alpha}
$$

where $\mathrm{v}^{\alpha}$ is the dislocation velocity on the $\alpha$ slip system. Equation (3.62) must be coupled with a dislocation velocity equation, which usually have the form of (Kocks $e t$ $a l ., 1975)$

$$
\mathrm{v}^{\alpha}=\mathrm{v}_{0} \exp \left[\frac{\mathrm{Q}_{\text {slip }}}{\mathrm{kb}}\left(1-\frac{\left|\tau^{\alpha}\right|}{\tau^{\mathrm{CSS}-\alpha}}\right)\right]
$$

where $\mathrm{v}_{0}$ is a reference velocity, $\mathrm{Q}_{\text {slip }}$ is an activation energy required to overcome obstacles to dislocation motion, and $\mathrm{k}$ is the Boltzmann's constant.

The advantage to the rate-dependent formulation is that all slip systems are potentially active. Thus there is no need to identify active slip systems. In addition, loading or unloading conditions are unnecessary. The draw back to the rate-dependent is that these flow rules often represent a stiff set of equations. As the equations get stiffer, calculation times greatly increase. 


\subsubsection{Work Hardening}

Kocks and Mecking (2003) described the current state of research on work hardening with the following statement: "A theory of work hardening is today as hopeless as ever; but a model of work hardening of pure fcc material we would consider now virtually available." In fact, a large number of work hardening models have been proposed that are applicable to pure fcc as well as other materials. One way the different work hardening models can be classified is those that explicitly account for dislocation densities and those that do not. Work hardening models that do not explicitly account for dislocation densities are still valid because they build in microstructure physics.

\subsubsection{Non Dislocation Density Based Work Hardening Models}

After initial yield, dislocation motion, or slip, continues on a slip system if $\tau^{\alpha}$ remains equal to the evolving $\tau^{\text {CSS- } \alpha}$. There are a number of different ways to model the change in $\tau^{\text {CSS- } \alpha}$. One way is to define the change in $\tau^{\text {CSS- } \alpha}$ via

$$
\dot{\tau}^{\mathrm{CSS}-\alpha}=\sum_{\beta=1}^{\mathrm{N}} \mathrm{h}^{\alpha \beta} \dot{\gamma}^{\beta},
$$

where $\mathrm{h}^{\alpha \beta}$ is the matrix containing the slip plane hardening moduli for the slip system $\alpha$ due to an increment of shear on slip system $\beta$. In this context, the diagonal components $\mathrm{h}^{\alpha \alpha}$ characterize self-hardening while the off diagonal terms, $\mathrm{h}^{\alpha \beta}$, characterize latent hardening. Since $\mathrm{h}^{\alpha \beta}$ defines the work hardening of a material, a lot of attention has been 
devoted to its development. One example of a relatively simple form of $h^{\alpha \beta}$ proposed by Hutchinson (1970) is

$$
\mathrm{h}^{\alpha \beta}=\left[\mathrm{q}+(1-\mathrm{q}) \delta_{\alpha \beta}\right] \mathrm{c}_{\mathrm{HU} 1}^{\beta}
$$

where $\mathrm{q}$ describes latent hardening $(1 \leq \mathrm{q} \leq 1.4), \delta_{\alpha \beta}$ is the Kronecker delta, and $\mathrm{c}_{\mathrm{HU} 1}^{\beta}$ is a material parameter. An example of a more complex form of $\mathrm{h}^{\alpha \beta}$ proposed by Bassani and $\mathrm{Wu}(1991)$ is

$$
\begin{gathered}
\mathrm{h}^{\alpha \alpha}=\left[\left(\mathrm{h}_{0}-\mathrm{h}_{\mathrm{s}}\right) \operatorname{sech}^{2}\left(\frac{\left(\mathrm{h}_{0}-\mathrm{h}_{\mathrm{s}}\right) \gamma^{\alpha}}{\tau_{\mathrm{sat}}-\tau_{0}}\right)+\mathrm{h}_{\mathrm{s}}\right]\left[1+\sum_{\beta \neq \alpha}^{\mathrm{N}} \mathrm{f}_{\alpha \beta} \tanh \left(\frac{\gamma^{\beta}}{\gamma_{0}}\right)\right] \\
\mathrm{h}^{\alpha \beta}=\mathrm{c}_{\mathrm{BW} 1} \mathrm{~h}^{\alpha \alpha}
\end{gathered}
$$

where $\mathrm{h}_{0}, \mathrm{~h}_{\mathrm{s}}, \tau_{0}, \tau_{\mathrm{sat}}, \mathrm{f}_{\alpha \beta}, \mathrm{c}_{\mathrm{BW} 1}$ and $\gamma_{0}$ are material constants, and $\gamma^{\alpha}$ is

$$
\gamma^{\alpha} \equiv \int\left|\dot{\gamma}^{\alpha}\right| \mathrm{dt}
$$

When a large number of slip systems are present, the $\mathrm{h}^{\alpha \beta}$ matrix can become quite large. For example, an FCC material has 12 slip systems and therefore $\mathrm{h}^{\alpha \beta}$ has 144 possible non-zero values. In order to simplify the approach to work hardening, researchers have developed simpler empirical descriptions of work hardening. Mathur and Dawson (1989) proposed that a single Voce based hardening function could represent hardening on all the slip systems. In their model, $\dot{\tau}^{*}$ represents an "effective grain hardness" and is a function of a "representative rate of shearing" $\dot{\gamma}^{*}$ as defined by

$$
\dot{\gamma}^{*}=\sum_{\alpha=1}^{N}\left|\dot{\gamma}^{\alpha}\right| .
$$

The Voce based hardening function is then 


$$
\dot{\tau}^{*}=\mathrm{c}_{\mathrm{MD} 1}\left(\frac{\tau_{\mathrm{sat}}-\tau}{\tau_{\mathrm{sat}}-\tau_{0}}\right) \dot{\gamma}^{*}
$$

where $\mathrm{c}_{\mathrm{MD} 1}$ is the initial hardening rate.

\subsubsection{Dislocation Density Based Work Hardening Models}

It is also possible to quantify work hardening as a function of microstructure. Assuming the flow stress for each slip system depends on the short-range dislocationdislocation interactions, the Taylor equation (Eq. 2.3) can be used to model work hardening. When the Taylor equation is written as a function of $\rho^{\mathrm{SSD}}$ only, evolution equations are needed to model the evolving SSD density. The Kocks-Mecking model (Estrin and Mecking, 1983) proposed a phenomenological evolution equation for $\rho^{\text {SSD }}$

$$
\frac{d \rho^{S S D-\alpha}}{d \gamma^{\alpha}}=c_{K M 1} \sqrt{\rho^{S S D-\alpha}}-c_{K M 2}\left(\rho^{S S D-\alpha}\right)
$$

where $\gamma^{\alpha}$ is the accumulated slip on slip system $\alpha$, The first term in Eq. (3.71) describes that dislocation storage is inversely proportional to mean free path, and the second term represents dislocation annihilation and cross-slip.

Zikery and Kao (1996) proposed a Taylor like equation for hardening that depended on $\rho^{\mathrm{I}}$

$$
\tau^{\mathrm{CSS}-\alpha}=\tau_{0}^{\alpha}+\mathrm{C}_{1} \mathrm{~Gb} \sqrt{\rho^{\mathrm{I}-\alpha}}+\mathrm{C}_{2} \mathrm{~Gb} \sqrt{\rho^{\mathrm{I}-\beta}} \quad \alpha \neq \beta
$$

where $\mathrm{C}_{1}$ and $\mathrm{C}_{2}$ are material constants. They proposed evolution coupled evolution equations for mobile $\left(\rho^{\mathrm{M}}\right)$ and immobile $\left(\rho^{\mathrm{I}}\right)$ dislocation densities 


$$
\begin{aligned}
& \dot{\rho}^{\mathrm{M}-\alpha}=\dot{\gamma}^{\alpha}\left[\frac{\mathrm{c}_{\mathrm{ZK} 1}}{\mathrm{~b}^{2}}\left(\frac{\rho^{\mathrm{I}-\alpha}}{\rho^{\mathrm{M}-\alpha}}\right)-\mathrm{c}_{\mathrm{ZK} 2} \rho^{\mathrm{M}-\alpha}-\frac{\mathrm{c}_{\mathrm{ZK} 3}}{\mathrm{~b}} \sqrt{\rho^{\mathrm{I}-\alpha}}\right] \\
& \dot{\rho}^{\mathrm{I}-\alpha}=\dot{\gamma}^{\alpha}\left[\mathrm{c}_{\mathrm{ZK} 2} \rho^{\mathrm{M}-\alpha}+\frac{\mathrm{c}_{\mathrm{ZK} 3}}{\mathrm{~b}} \sqrt{\rho^{\mathrm{I}-\alpha}}-\mathrm{c}_{\mathrm{ZK} 4} \rho^{\mathrm{I}-\alpha}\right]
\end{aligned}
$$

where $\mathrm{c}_{\mathrm{ZK} 1}$ is related to mobile dislocation production from dislocation sources, $\mathrm{c}_{\mathrm{ZK} 2}$ is related to mobile dislocation trapping due forest interactions and cross-slip around obstacles, $\mathrm{c}_{\mathrm{ZK} 3}$ is related to immobilization of mobile dislocations, $\mathrm{c}_{\mathrm{ZK} 4}$ is related to the rearrangement and annihilation of immobile dislocations.

The effect of GNDs on work hardening in the Taylor equation is represented by a scalar, $\rho^{\mathrm{GND}}$. However, Nye's dislocation tensor, Eq. (3.18), describes the local GND state as a tensor with 9 components. The distribution of the GNDs within the volume element represented by the dislocation tensor depends on the crystallography of the material. Assuming that the dislocation density can be represented as a line length per volume, Nye's dislocation tensor can be rewritten as (Arsenlis and Parks, 1999)

$$
\mathbf{g}=\sum_{\alpha=1}^{\mathrm{N}_{\mathrm{sys}}} \rho^{\mathrm{GND}-\alpha} \mathbf{b}^{\alpha} \cdot \boldsymbol{\zeta}^{\alpha}
$$

where $\mathbf{b}$ is the burgers vector of a dislocation with a tangent line direction $\zeta$. If it is further assumed that all the dislocations are straight, then there are only 3 possible dislocation types per slip plane: 1 screw and 2 edge. Take the case of simple cubic: there are 3 slip planes with 3 dislocation types per slip plane. In this case Eq. (3.75) represents a linear system of 9 equations and 9 unknowns. For an fcc lattice, there are 12 slip systems, each with 3 possible straight dislocations. In this case Eq. (3.75) represents an underdetermined system of equations: 9 equations and 36 unknowns. There are a number of numerical methods (for example the simplex method of linear programming) that can 
be used to solve underdetermined system of equations. However, these methods are computationally expensive and have not been implemented in a crystal plasticity model. A less computationally expensive way to determine $\rho^{\mathrm{GND}}$ from Nye's tensor is to project the dislocation tensor onto each of the slip planes via (Bammann, 2001)

$$
\rho^{\mathrm{GND}-\alpha}=\frac{\left|\mathbf{g}:\left(\mathbf{s}^{\alpha} \otimes \mathbf{m}^{\alpha}\right)\right|}{\mathrm{b}} .
$$

With this approach, information on the GND state is lost and the accuracy of this approach is questionable.

\subsection{Constitutive Relations}

In most crystal plasticity models, the term "constitutive relations" applies to the different types of stress-strain relationships employed to model crystalline metals. The two categories of stress-strain relationships used to solve the elastic-plastic crystal plasticity problem are hypo-elastic plasticity and hyper-elastic plasticity.

\subsubsection{Hypo-elasticity}

Hypo-elastic plastic models use a rate based stress-strain (or deformation) constitutive equations. If it is assumed that the crystal's elasticity is unaffected by slip, then following Hill and Rice (1972) and Asaro and Rice (1977) elasticity can be represented by

$$
\boldsymbol{\sigma}^{*}=\underset{\sim}{L}: \mathbf{D}^{\mathbf{e}}
$$


where $\stackrel{\nabla}{\sigma}^{*}=$ a Cauchy objective (co-rotational) stress rate, $\underset{\sim}{\boldsymbol{L}}$ is the fourth order elastic modulus tensor, and $\mathbf{D}^{\mathbf{e}}$ is the elastic deformation rate tensor. It is further assumed that the total deformation rate, $\mathbf{D}$, can be decomposed additively into elastic and plastic parts:

$$
\mathbf{D}=\mathbf{D}^{\mathbf{e}}+\mathbf{D}^{\mathbf{P}}
$$

where $\mathbf{D}^{\mathbf{P}}$ is the plastic deformation rate tensor.

Co-rotational derivates are used in the constitutive equation to ensure the preservation of objectivity. Researchers have developed a number of different corotational derivatives, all of which can be used in hypo-elastic plastic models. An example of a commonly used co-rotational derivate is the Jaumann derivative $\left(\sigma^{* J}\right)$ defined on the axes that rotates with the lattice

$$
\sigma^{\nabla^{* J}}=\dot{\sigma}-\Omega^{\mathrm{e}} \cdot \sigma+\sigma \cdot \Omega^{\mathrm{e}}
$$

where $\dot{\sigma}$ is the ordinary time derivative of the Cauchy stress, $\boldsymbol{\Omega}^{\mathrm{e}}$ is the elastic spin rate, and $\boldsymbol{\sigma}$ is the Cauchy stress. It is also possible to define a Jaumann derivative $\left(\stackrel{\nabla}{\sigma^{\mathrm{J}}}\right)$ on the axes that rotates with the material via the formula

$$
\stackrel{\nabla}{\sigma^{\mathrm{J}}}=\dot{\sigma}-\Omega \cdot \sigma+\sigma \cdot \Omega
$$

where $\boldsymbol{\Omega}$ is the total spin rate.

Hypo-elastic plastic models are computationally efficient and used in most rateindependent formulations. The assumption that $\mathbf{D}$ can be decomposed additively limits these types of models to small elastic strains but large rotations. Even with this limitation, the hypo-elastic plastic approach is appropriate for most engineering materials, 
including metals, where elastic strains remain small. Another weakness of the hypoelastic plastic approach lies in the somewhat arbitrary choice of an objective stress rate.

\subsubsection{Hyper-elasticity}

The stress-strain relationship in hyper-elastic plastic models is derived from a free energy function that depends on strains. Starting with the argument of Mandel and others, the Helmholtz free energy function, $\psi$, depends on $\boldsymbol{\varepsilon}^{\mathbf{e}}$, various structure properties $\left(\boldsymbol{\alpha}_{\mathrm{i}}\right)$, and T:

$$
\psi=\bar{\psi}\left(\varepsilon^{\mathrm{e}}, \boldsymbol{\alpha}_{\mathrm{i}}, \mathrm{T}\right)
$$

The $1^{\text {st }}$ and $2^{\text {nd }}$ laws of thermodynamics define that the thermodynamically conjugate force to $\mathbf{E}^{\mathbf{e}}$ has the form

$$
\frac{\partial \psi}{\partial \varepsilon^{\mathrm{e}}}=\sigma
$$

If the Helmholtz free energy is assumed to have a quadratic dependence on elastic strain

$$
\psi=\frac{1}{2} \underset{\sim}{\boldsymbol{L}}:\left(\boldsymbol{\varepsilon}^{\mathrm{e}}\right)^{2}
$$

then the linear stress-strain law

$$
\sigma=\underset{\sim}{L}: \varepsilon^{\mathrm{e}}
$$

can be derived.

An advantage of this formulation is that stresses are computed directly from elastic strains. Since the constitutive law directly relates stresses and strains and not stress rates and strain rates, objectivity is automatically satisfied (assuming that the free 
energy is determined by quantities that are invariant under a rigid body rotation). Thus there is no need for co-rotational derivatives.

\subsection{Numerical Integration}

All crystal plasticity use numerical integration to update the state of the material over a time step $\Delta \mathrm{t}$. The state of the material is known at the beginning of the time step, time $t$, and numerical integration of the appropriate differential equations yields the state

of the material at the end of the time step, $t+\Delta t$. Many different integration schemes have been applied to and developed for crystal plasticity. These different integration schemes fall broadly into two categories: explicit integration or implicit integration.

\subsubsection{Explicit Integration}

In the explicit approach, quantities associated with the material state at time $t$ are used to update quantities associated with the material state at time $t+\Delta t$. Using known quantities at time $\mathrm{t}$ as a starting point makes this approach direct and simple to implement. The down side is that this approach is numerically unstable when $\Delta \mathrm{t}$ is larger than some critical time step, $\Delta \mathrm{t}_{\text {crit }}$. The $\Delta \mathrm{t}_{\text {crit }}$ connected with the stiff, non-linear equations used in most crystal plasticity models is usually small. Thus, small time steps must be taken to prevent oscillations in the predicted stress state. 


\subsubsection{Implicit Integration}

In the implicit approach, quantities associated with the material state at time $t+\Delta t$ are used to update quantities associated with the material state at time $t+\Delta t$. Implicit integration schemes are based on isolating and solving for a specific quantity associated with the material state at time $t+\Delta t$. Then using this value to update all the other quantities associated with the material state at time $t+\Delta t$. For example, in a rate dependent, hyper-elastic solid, a level function for each slip system, $\mathrm{f}^{\alpha}$, is defined as (Cuitino and Ortiz, 1992)

$$
\mathrm{f}^{\alpha}=\tau_{\mathrm{t}+\Delta t}^{\alpha}-\dot{\tau}_{\mathrm{t}+\Delta t}^{\alpha}\left|\frac{\dot{\gamma}_{\mathrm{t}+\Delta t}^{\alpha}}{\dot{\gamma}_{0}}\right| \operatorname{sgn}\left(\dot{\gamma}^{\alpha}\right)=0 .
$$

Once $\tau_{t+\Delta t}^{\alpha}$ and $\dot{\tau}_{t+\Delta t}^{\alpha}$ are defined in terms of $\dot{\gamma}_{t+\Delta t}^{\alpha}$, then $\dot{\gamma}_{t+\Delta t}^{\alpha}$ can be determined and used to update all the quantities at $t+\Delta t$. The advantage of this approach is that numerical stability is guaranteed; however, the numerical algorithms (like the Newton-Raphson method) needed for this approach are more complex.

\subsection{Calculating Gradient Quantities in Crystal Plasticity}

In many crystal plasticity models, gradient quantities are calculated using shape function derivatives. While the shape function approach is well established in the FEM community, Gao and Huang (2001) developed the basis for an alternative technique to approximate the gradient with integrals. 


\subsubsection{Gradient Quantities Determined by Derivatives of Shape Functions}

The numerical framework for determining gradient quantities with shape function derivatives in crystal plasticity is the same approach FEM codes use to calculate gradient quantities like strain and the velocity gradient. A 1 dimensional example in which strain ( $\varepsilon$ ) and the gradient of dislocation density $(\nabla \rho)$ are calculated using the shape function derivative method follows. Consider a bar element with a length $\ell$ where the displacements (d) and the dislocation densities ( $\rho)$ are known at each end as shown in Figure 3.6.

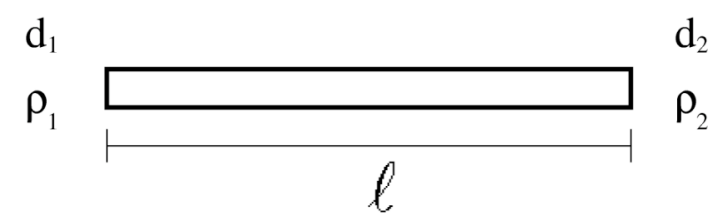

Figure 3.6 - 1D Bar Element

The displacement and dislocation density at any point along the bar can be interpolated from the values at each end of the bar with shape functions. Assuming that values vary linearly over the length of the bar, the two shape functions, $\mathrm{N}_{1}$ and $\mathrm{N}_{2}$, are

$$
\mathrm{N}_{1}=1-\frac{\mathrm{x}}{\ell}, \quad \mathrm{N}_{2}=\frac{\mathrm{x}}{\ell}
$$

The derivative of the two shape functions with respect to $\mathrm{x}$ is

$$
\frac{\mathrm{dN}_{1}}{\mathrm{dx}}=-\frac{1}{\ell}, \quad \frac{\mathrm{dN}_{2}}{\mathrm{dx}}=\frac{1}{\ell}
$$

The shape function derivatives make up the [B] matrix as

$$
[\mathrm{B}]=\left[\begin{array}{ll}
-\frac{1}{\ell} & \frac{1}{\ell}
\end{array}\right] \text {. }
$$


At this point, determining the gradient becomes a linear algebra problem. Strain, which is the gradient in displacement, is calculated by

$$
\varepsilon=[\mathrm{B}][\mathrm{d}]=\left[\begin{array}{cc}
-\frac{1}{\ell} & \frac{1}{\ell}
\end{array}\right]\left[\begin{array}{l}
\mathrm{d}_{1} \\
\mathrm{~d}_{2}
\end{array}\right]=-\frac{\mathrm{d}_{1}}{\ell}+\frac{\mathrm{d}_{2}}{\ell},
$$

and $\nabla \rho$ is calculated by

$$
\nabla \rho=[B][\rho]=\left[\begin{array}{cc}
-\frac{1}{\ell} & \frac{1}{\ell}
\end{array}\right]\left[\begin{array}{l}
\rho_{1} \\
\rho_{2}
\end{array}\right]=-\frac{\rho_{1}}{\ell}+\frac{\rho_{2}}{\ell} .
$$

Note that since the shape functions are linear across the bar, the gradient quantities are constant along the bar.

The shape function derivative approach is used in crystal plasticity models for computational reasons. The FEM code has already calculated [B] making the gradient calculation in the crystal plasticity model just a matrix dot product. The disadvantage to this approach lies in the thermodynamics of gradient models: each gradient quantity has a conjugate force or stress that the model must account for. (See Chapter 3.2.2 for details on the thermodynamics of gradient ISV models.)

\subsubsection{Gradient Quantities Approximated with Integrals}

Gao and Huang (2001) developed an approach by which the strain gradient was estimated with a non-local integral. The derivation for this approximation is based on a Taylor series. Consider a Taylor series expansion of $\mathrm{H}$ in the neighborhood of point $\mathbf{x}$

$$
\mathrm{H}(\mathbf{x}+\xi)=\mathrm{H}(\mathbf{x})+\nabla \mathrm{H}(\mathbf{x}) \xi+\mathbf{O}\left(|\xi|^{2}\right)
$$


where $\xi$ represents the local coordinates centered at $\mathbf{x}$. Multiplying each side by $\xi^{5}$ and integrating each term over a small representative volume $\mathrm{V}_{\text {cell }}$ containing $\mathbf{x}$, resulted in

$$
\int_{\mathrm{V}_{\text {cell }}} \mathrm{H}(\mathbf{x}+\xi) \otimes \xi \mathrm{d} \xi=\int_{\mathrm{V}_{\text {cell }}} \mathrm{H}(\mathbf{x}) \otimes \xi \mathrm{d} \xi+\int_{\mathrm{V}_{\text {cell }}} \nabla \mathrm{H}(\mathbf{x}) \xi \otimes \xi \mathrm{d} \xi
$$

The integral approximation of the gradient term then became

$$
\{\nabla \mathrm{H}\}^{\mathrm{GH}}(\mathbf{x})=\int_{\mathrm{V}_{\mathrm{cell}}}(\mathrm{H}(\mathbf{x}+\xi)-\mathrm{H}(\mathbf{x})) \otimes \xi \mathrm{d} \xi \quad\left(\int_{V_{\text {cell }}} \xi \otimes \xi \mathrm{d} \xi\right)^{-1}
$$

$\{\nabla \mathrm{H}\}^{\mathrm{GH}}$ will asymptotically approach the true gradient, $\nabla \mathrm{H}$, as $\mathrm{V}_{\text {cell }} \rightarrow 0$.

$\{\nabla \mathrm{H}\}^{\mathrm{GH}}$ as defined in Eq. (3.93) is not a true non-local quantity. By definition, a non-local integral has an attenuation function that is applied to the entire material body. In the expression for $\{\nabla \mathrm{H}\}^{\mathrm{GH}}$ there is no attenuation function, which causes the integral expression for $\{\nabla \mathrm{H}\}^{\mathrm{GH}}$ to diverge. Gao and Huang minimized the divergence problem by keeping the integration volume, $\mathrm{V}_{\text {cell }}$, small.

The integral approach proposed by Gao and Huang can be modified and cast as a non-local integral by applying a proper attenuation function. Applying the Gaussian attenuation function to the Gao and Huang approach results in

$$
\{\nabla \mathrm{H}\}^{\text {Gauss }}(\mathbf{x})=\int_{\mathrm{V}_{\text {cell }}}(\mathrm{H}(\mathbf{x}+\boldsymbol{\xi})-\mathrm{H}(\mathbf{x})) \otimes \xi \operatorname{Exp}\left(-\frac{\|\xi\|^{2}}{2 \omega^{2}}\right) \mathrm{d} \xi\left(\int_{\mathrm{v}_{\text {cell }}} \xi \otimes \xi \operatorname{Exp}\left(-\frac{\|\xi\|^{2}}{2 \omega^{2}}\right) \mathrm{d} \xi\right)^{-1}
$$

where $\|\xi\|=\sqrt{\left(\xi_{1}\right)^{2}+\left(\xi_{2}\right)^{2}+\left(\xi_{3}\right)^{2}}$. The volume normalizing term, $(1 \sqrt{2 \pi})^{- \text {Dim }}$, doesn't appear in the expression for $\{\nabla \mathrm{H}\}^{\text {Gauss }}$ because it cancels out of the numerator and

\footnotetext{
${ }^{5}$ The need to multiply each term by $\xi$ is seen in Eq. (3.92): when $\xi$ is centered within $\mathrm{V}_{\text {cell }}, \int_{\mathrm{V}_{\text {cell }}} \xi \mathrm{d} \mathbf{V}=0$ while $\int_{\mathrm{V}_{\text {cell }}} \xi \otimes \xi \mathrm{d} \mathbf{V} \neq 0$.
} 
denominator. It is also possible to use an attenuation function that depends on $\|\xi\|$ rather than $\|\xi\|^{2}$ such as

$$
\Theta(\mathbf{r})=\operatorname{Exp}\left(-\frac{\|\xi\|}{\omega}\right)
$$

In this case, in integral approximation to the gradient becomes

$$
\{\nabla \mathrm{H}\}^{\operatorname{Exp}}(\mathbf{x})=\int_{V_{\text {cell }}}(\mathrm{H}(\mathbf{x}+\xi)-\mathrm{H}(\mathbf{x})) \otimes \xi \operatorname{Exp}\left(-\frac{\|\xi\|}{\omega}\right) \mathrm{d} \xi\left(\int_{\mathrm{V}_{\text {cell }}} \xi \otimes \xi \operatorname{Exp}\left(-\frac{\|\xi\|}{\omega}\right) \mathrm{d} \xi\right)^{-1}
$$

Mathematically, $\omega$ defines the manner in which the attenuation function approaches zero,

effectively limiting the integration volume. As $\omega$ approaches $\infty,\{\nabla H\}^{\text {Gauss }}$ and $\{\nabla \mathrm{H}\}^{\text {Exp }}$ both asymptotically approach the value of the true $\nabla \mathrm{H}$. 


\section{CHAPTER 4}

\section{IMPLEMENTATION OF THE LOCAL AND NON-LOCAL CYRSTAL PLASTICITY MODEL}

In order to model a wide range of grain sizes within a material, both a local and non-local model are needed. When the mechanical response of a material is length scale independent, a local model is sufficient. However, when the mechanical response of a material is grain size dependent, a non-local model is needed to account for the grain size effects.

In this chapter, the details for both the local and non-local crystal plasticity model implemented in this research are presented. As discussed in Chapter 3, there are a number of different ways to implement crystal plasticity into a finite element framework. Considering the advantages and disadvantages of the various features discussed in Chapter 3, both a local and non-local crystal plasticity model were developed. The major features of each model are summarized in Table 4.1.

Table 4.1 - Features of the Local and Non-local Crystal Plasticity Models

\begin{tabular}{|l|c|c|}
\hline & $\begin{array}{c}\text { Local Crystal Plasticity } \\
\text { Model }\end{array}$ & Non-local Crystal Plasticity Model \\
\hline Kinematics & Polycrystal $\mathbf{F}^{\mathbf{e}} \mathbf{F}^{\mathbf{P}}$ & Polycrystal $\mathbf{F}^{\mathbf{e}} \mathbf{F}^{\mathbf{P}}$ \\
Kinetics & Rate-Dependent & Rate-Dependent \\
Constitutive Relation & Hyper-elastic plastic & Hyper-elastic plastic \\
Hardening & Taylor model w/SSDs & Taylor model w/SSDs and GNDs \\
Crystal Plasticity & Not Applicable & Integral Approximation technique \\
Gradient Calculation & & \\
Numerical Integration & Explicit - Forward Euler & Explicit - Forward Euler \\
\hline
\end{tabular}


Both crystal plasticity models were then incorporated into Sandia National Laboratory's finite element analysis code, JAS3D (Biffle, 1987). JAS3D is a 3D finite element code designed to solve large quasi-static nonlinear mechanics problems. Within JAS3D, the continuum equations are interatively solved using a dynamic relaxation algorithm.

\subsection{Role of Crystal Plasticity Models within JAS3D}

The primary role of the crystal plasticity model within the finite element code structure is to calculate the stress state and to update the corresponding state variables for a particular deformation state. The interaction of the finite element code and the crystal plasticity model is illustrated in Figure 4.1. The FEM simulation starts with the initialization routine, which initializes all the necessary variables. Once initialization is complete, the FE code deforms the material. The finite element code "guesses" or predicts the deformation state at the end of the time step $\left(\mathbf{F}_{t+\Delta t}\right)$ given the boundary conditions and the previous stress and deformation states at the beginning of the time step $\left(\sigma_{t}\right.$ and $\left.\mathbf{F}_{t}\right)$. This predicted deformation state is then passed to the crystal plasticity model, which calculates the corresponding Cauchy stress state at the end of the time step, $\sigma_{t^{+} \Delta t}$. The crystal plasticity model returns $\sigma_{t^{+} \Delta t}$ to the finite element code, which then

checks if the stress state satisfies equilibrium. If it does, the "guessed" deformation state and the corresponding stress state are accepted and the code moves on to the next time step. If not, the code calculates another $\mathbf{F}_{\mathrm{t}+\Delta t}$, which is again passed to the crystal plasticity model. This process continues until a stress state associated with a "guessed" deformation satisfies equilibrium. 


\section{Finite Element Code $\quad$ Crystal Plasticity Model}

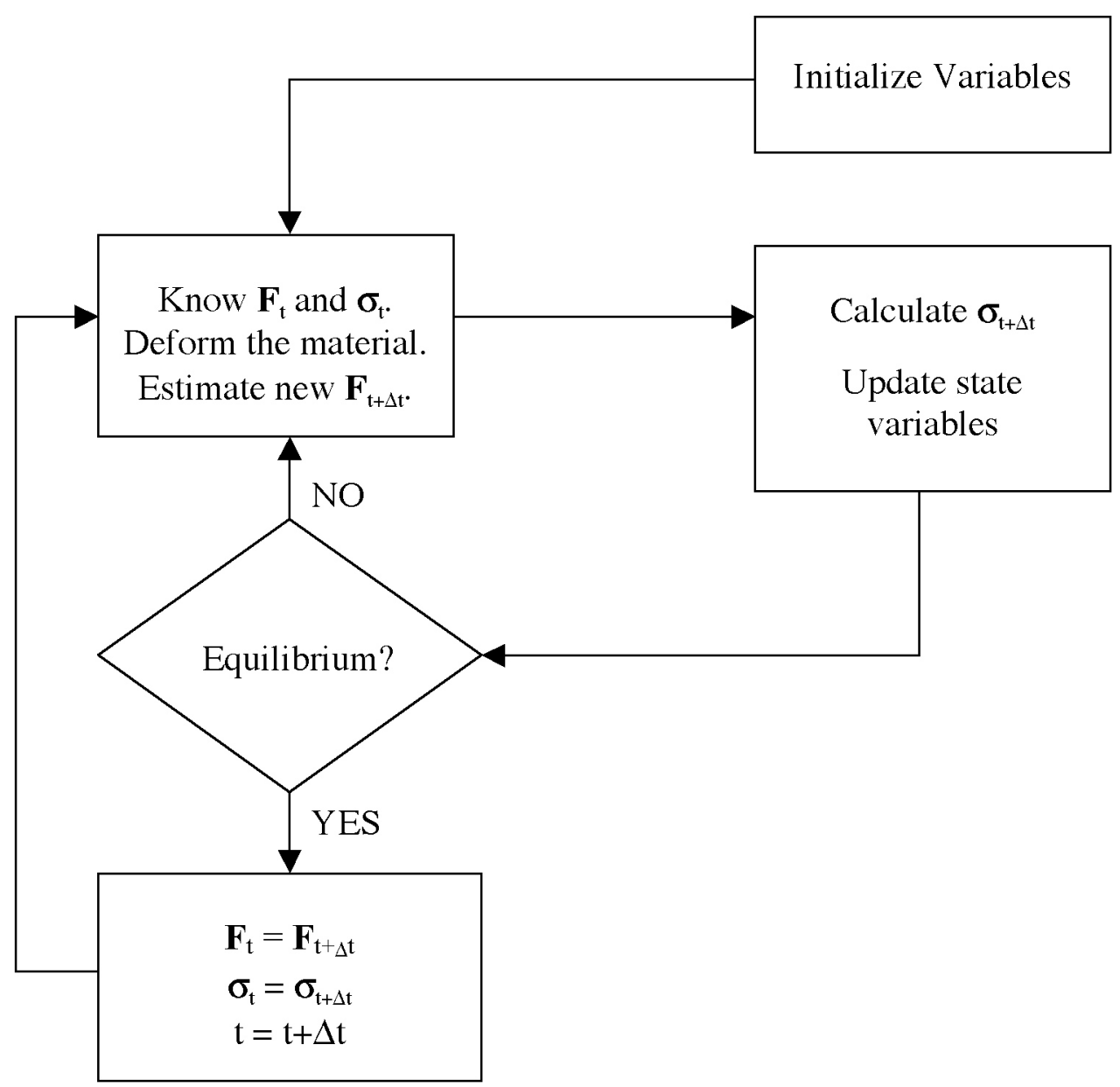

Figure 4.1 - Relationship between the Finite Element Code and the Material Model. Quantities at the beginning of the time step have a subscript $t$ and quantities at the end of the time step $\Delta \mathrm{t}$ have a subscript $\mathrm{t}+\Delta \mathrm{t}$. 
Both the local and non-local crystal plasticity model was incorporated into Sandia's finite element analysis code, JAS3D (Biffle, 1987) as a standard subroutine. Incorporating the local model into JAS3D was straightforward since JAS3D was designed to run local material models. The non-local model was implemented into JAS3D in a non-standard manner. Each call to the non-local model subroutine required that neighbor information about $\mathbf{F}^{\mathbf{P}}, \mathbf{R}_{\mathrm{h}}^{\mathrm{e}}$, and distances between elements be available. Unfortunately, JAS3D was not designed with this capability. For memory minimization and computational efficiency, JAS3D breaks the mesh into element blocks. Each element block was passed to the material subroutine separately meaning no information could be passed between element blocks. In order to make neighbor information available for the non-local model, the entire mesh was passed to the material subroutine as one element block. The disadvantage to this approach was twofold. First it made parallelization of the non-local model impossible, and second this approach was memory intensive and limited the mesh to approximately 50,000 elements.

Unlike most finite element codes, JAS3D uses a single integration point at the element centroid as the basis for its integration scheme. Hourglass control, based on the work of Flanagan and Belytschko (1981), is needed to deal with possible zero energy modes that may arise as a result of the single point approach. With only a single integration point, the evaluation of the non-local integrals within the non-local model is greatly simplified. 


\subsection{Local Crystal Plasticity Model}

The local crystal plasticity model was developed to provide a framework from which to build the non-local crystal plasticity model. Thus the local model was designed in such a way as to facilitate the implementation the non-local integral based hardening. For this reason, the local crystal plasticity model presented here is not as sophisticated and computationally efficient as other local crystal plasticity models in the literature. Yet, this local crystal plasticity model was still a useful tool in this research project because the model was built on sound physical principles (rate-dependent kinetics, hyperelastic plastic constitutive equation, Taylor hardening).

The numerical algorithm used in the local crystal plasticity model can be broken into 4 parts: 1) initialization, 2) stress update, 3) hardening, and 4) stability check/subincrementation. A description of each step follows.

\subsubsection{Initialization}

The initialization routine for the local model defines the crystallographic orientations and the material properties of the material. The orientation of each grain's crystal lattice is determined by generating a set of 3 random Euler angles. These 3

random Euler angles $\left(\phi_{1}, \phi_{2}, \phi_{3}\right)$ are calculated from three random numbers $\left(\#_{\mathrm{R} 1}, \#_{\mathrm{R} 2}, \#_{\mathrm{R} 3}\right)$ between 0 and 1 via

$$
\phi_{1}=2 \pi \#_{\mathrm{R} 1}, \phi_{2}=\cos ^{-1}\left(2 \#_{\mathrm{R} 2}-1\right), \phi_{3}=2 \pi \#_{\mathrm{R} 3} .
$$


There are multiple convention for converting Euler angles into a rotation matrix. Notice that Eq. (4.1) does not define which axes $\phi_{1}, \phi_{2}$, and $\phi_{3}$ are rotated or in which order they are made. The convention used to convert $\phi_{1}, \phi_{2}$, and $\phi_{3}$ into a rotation matrix was the following: the first rotation was about the z-axis by an angle $\phi_{1}$, the second rotation ws about the $\mathrm{x}$-axis by an angle $\phi_{2}$, and the third rotation ws about the rotated z-axis by an angle $\phi_{3}$. When these three rotations were combined, they yielded the following rotation matrix:

$$
\mathbf{R}_{\mathbf{h}}^{\mathbf{e}}=\left(\begin{array}{ccc}
\cos \phi_{1} \cos \phi_{3}-\sin \phi_{1} \cos \phi_{2} \sin \phi_{3} & \sin \phi_{1} \cos \phi_{3}+\cos \phi_{1} \cos \phi_{2} \sin \phi_{3} & \sin \phi_{2} \sin \phi_{3} \\
-\cos \phi_{1} \sin \phi_{3}-\sin \phi_{1} \cos \phi_{2} \cos \phi_{3} & -\sin \phi_{1} \sin \phi_{3}+\cos \phi_{1} \cos \phi_{2} \cos \phi_{3} & \sin \phi_{2} \cos \phi_{3} \\
\sin \phi_{1} \sin \phi_{2} & -\cos \phi_{1} \sin \phi_{2} & \cos \phi_{2}
\end{array}\right)
$$

The grain orientations represented by $\mathbf{R}_{\mathbf{h}}^{\mathbf{e}}$ are then used to initialize the slip systems and the elasticity tensor in accordance with the polycrystal $\mathbf{F}^{\mathbf{e}} \mathbf{F}^{\mathbf{P}}$ kinematics. The slip direction vector in the single crystal configuration $\left(\mathbf{s}_{\mathbf{S}-\mathbf{x} \text { tal }}^{\alpha}\right)$, the slip plane normal vector in the single crystal configuration $\left(\mathbf{m}_{\mathbf{S}-\mathbf{x t a l}}^{\alpha}\right)$, and the elasticity tensor in the single crystal configuration $\left(\underset{\sim}{\boldsymbol{L}_{\text {S-xtal }}}\right)$ are pushed forward to the reference configuration with $\mathbf{F}_{\mathbf{h}}^{\mathbf{e}}$. However, $\mathbf{F}_{\mathbf{h}}^{\mathbf{e}}=\mathbf{R}_{\mathbf{h}}^{\mathbf{e}}$ meaning the single crystal configuration quantities are just rotated into the reference configuration by

$$
\begin{gathered}
\mathbf{S}^{\alpha}=\mathbf{R}_{\mathrm{h}}^{\mathrm{e}} \cdot \mathbf{S}_{\mathrm{S}-\mathrm{xtal}}^{\alpha} \\
\mathbf{M}^{\alpha}=\mathbf{R}_{\mathrm{h}}^{\mathrm{e}} \cdot \mathbf{m}_{\mathrm{S}-\mathrm{xtal}}^{\alpha} \\
\underset{\sim}{\boldsymbol{L}}=\mathbf{R}_{\mathrm{h}}^{\mathrm{e}} \cdot \mathbf{R}_{\mathrm{h}}^{\mathrm{e}} \cdot \underset{\sim}{\boldsymbol{L}_{\mathrm{S}-\mathrm{xtal}}} \cdot\left(\mathbf{R}_{\mathrm{h}}^{\mathrm{e}}\right)^{\mathrm{T}} \cdot\left(\mathbf{R}_{\mathrm{h}}^{\mathrm{e}}\right)^{\mathrm{T}} .
\end{gathered}
$$


In fcc crystals, $\mathbf{s}_{\mathbf{S}-\mathbf{x t a l}}^{\alpha}$ is composed of the $<110>$ family of directions normalized to unit length, and $\mathbf{m}_{\text {S-xtal }}$ is made up of the directions normal to the $\{111\}$ family of planes also normalized to unit length.

Once the crystal lattice is defined, the material properties are initialized. The material body is assumed to be stress free in the reference configuration making

$$
\mathbf{F}_{\mathrm{t}=0}=\mathbf{I}, \mathbf{F}_{\mathrm{t}=0}^{\mathrm{e}}=\mathbf{I}, \mathbf{F}_{\mathrm{t}=0}^{\mathbf{P}}=\mathbf{I}{ }^{6}
$$

The initial SSD density $\left(\rho_{\mathrm{t}=0}^{\mathrm{SSD}-\alpha}\right)$ is related to the initial hardening state (represented by $\left.\tau_{\mathrm{t}=0}^{\mathrm{CSS}-\alpha}\right)$. Since $\tau_{\mathrm{t}=0}^{\text {CSS- } \alpha}$ is an input parameter, $\rho_{\mathrm{t}=0}^{\text {SSD- } \alpha}$ is defined by via Taylor's equation

$$
\rho_{\mathrm{t}=0}^{\mathrm{SSD}-\alpha}=\left(\frac{\tau_{\mathrm{t}=0}^{\mathrm{CSS}-\alpha}}{\mathrm{CG}_{110} \mathrm{~b}}\right)^{2}
$$

where $\mathrm{G}_{110}$ is the shear modulus in the 110 direction. The bulk shear modulus, $\mathrm{G}$, is commonly used in Eq. (4.7) rather than the directional $\mathrm{G}_{110}$ shear modulus. However, at the grain level the directional shear modulus in the direction of slip is a better choice since the elastic constants of a grain are anisotropic. In addition, it is assumed that $\tau_{\mathrm{t}=0}^{\mathrm{CSS}-\alpha}$ and therefore $\rho_{\mathrm{t}=0}^{\mathrm{SSD}-\alpha}$ is equivalent on each slip system.

\subsubsection{Stress Update (McGinty, 2001)}

The explicit numerical integration scheme employed in the local model uses quantities at time $t$ to update the Cauchy stress and other quantities at time $t+\Delta t$. It is numerically advantageous to cast much of the stress update algorithm in the intermediate

\footnotetext{
${ }^{6}$ Initial quantities have a subscript $\mathrm{t}=0$ rather than just 0 to differentiate them from constants, which might have a subscript 0 .
} 
configuration defined by the $\mathbf{F}^{\mathbf{e}}$ and $\mathbf{F}^{\mathbf{P}}$ based kinematics. Within these kinematics plastic deformation does not change the crystal structure of the material making $\hat{\mathbf{s}}^{\alpha}, \hat{\mathbf{m}}^{\alpha}, \underset{\sim}{\hat{\boldsymbol{L}}}$ time invariant ${ }^{7}$. All time invariant quantities are expressed without a time subscript.

The explicit algorithm starts by projecting the Cauchy stress at time t onto each of the slip systems in the current configuration by

$$
\tau_{t}^{\alpha}=\sigma_{t}:\left(\mathbf{s}_{t}^{\alpha} \otimes \mathbf{m}_{t}^{\alpha}\right)
$$

The rate dependent viscoplastic flow rule (Eq. 3.59) specifies $\dot{\gamma}_{t}^{\alpha}$ as a function of the resolved shear stress $\left(\tau_{t}^{\alpha}\right)$ and the critical resolved shear stress needed for plastic flow $\left(\tau_{t}^{\mathrm{CSS}-\alpha}\right)$

$$
\dot{\gamma}_{t}^{\alpha}=\dot{\gamma}_{0}\left|\frac{\tau_{t}^{\alpha}}{\tau_{t}^{C S S}-\alpha}\right|^{\frac{1}{m}} \operatorname{sgn}\left(\tau_{t}^{\alpha}\right)
$$

Because $\dot{\gamma}_{t}^{\alpha}$ is configuration independent, $\dot{\gamma}_{t}^{\alpha}$ is used to compute the plastic velocity gradient in the intermediate configuration $\left(\hat{\mathbf{L}}_{t}^{\mathbf{P}}\right)$ via

$$
\hat{\mathbf{L}}_{\mathrm{t}}^{\mathbf{P}}=\sum_{\alpha=1}^{\mathrm{N}} \dot{\gamma}_{\mathrm{t}}^{\alpha}\left(\hat{\mathbf{s}}^{\alpha} \otimes \hat{\mathbf{m}}^{\alpha}\right) .
$$

The next step in the stress update algorithm is to integrate $\dot{\mathbf{F}}^{\mathbf{P}}=\hat{\mathbf{L}}^{\mathbf{P}} \cdot \mathbf{F}^{\mathbf{P}}$ to find $\mathbf{F}_{t+\Delta t}^{\mathbf{P}}$. This integration is done using an exponential function (Weber and Anand, 1990)

$$
\mathbf{F}_{t+\Delta t}^{\mathbf{P}}=\exp \left(\hat{\mathbf{L}}_{\mathrm{t}}^{\mathbf{P}} \Delta \mathrm{t}\right) \cdot \mathbf{F}_{\mathrm{t}}^{\mathbf{P}}
$$

where

\footnotetext{
${ }^{7}$ Another consequence of the assumption that plastic deformation does not affect the crystal structure is that $\mathbf{S}^{\alpha}=\hat{\mathbf{s}}^{\alpha}, \mathbf{M}^{\alpha}=\hat{\mathbf{m}}^{\alpha}$, and $\underset{\sim}{\boldsymbol{L}}=\hat{\sim}$.
} 


$$
\exp \left(\hat{\mathbf{L}}_{\mathrm{t}}^{\mathbf{P}} \Delta \mathrm{t}\right)=\mathbf{I}+\frac{\sin \phi}{\phi} \hat{\mathbf{L}}_{\mathrm{t}}^{\mathbf{P}} \Delta \mathrm{t}+\frac{1-\cos \phi}{\phi^{2}}\left(\hat{\mathbf{L}}_{\mathrm{t}}^{\mathbf{P}} \cdot \hat{\mathbf{L}}_{\mathrm{t}}^{\mathbf{P}}\right) \Delta \mathrm{t}^{2}
$$

and

$$
\phi=\sqrt{0.5\left(\hat{\mathbf{L}}_{\mathrm{t}}^{\mathbf{P}}: \hat{\mathbf{L}}_{\mathrm{t}}^{\mathbf{P}}\right)} \Delta \mathrm{t}
$$

$\mathbf{F}_{t+\Delta t}^{\mathbf{e}}$ is found by combining the newly calculated $\mathbf{F}_{t+\Delta t}^{\mathbf{P}}$ with $\mathbf{F}_{t+\Delta t}$ passed to the material model by the FEM code

$$
\mathbf{F}_{\mathrm{t}+\Delta \mathrm{t}}^{\mathbf{e}}=\mathbf{F}_{\mathrm{t}+\Delta \mathrm{t}} \cdot\left(\mathbf{F}_{\mathrm{t}+\Delta \mathrm{t}}^{\mathbf{P}}\right)^{-1}
$$

The Green strain tensor in the intermediate configuration is then calculated by

$$
\hat{\mathbf{E}}_{\mathrm{t}+\Delta \mathrm{t}}^{\mathrm{e}}=0.5\left[\left(\mathbf{F}_{\mathrm{t}+\Delta \mathrm{t}}^{\mathrm{e}}\right)^{\mathrm{T}} \cdot \mathbf{F}_{\mathrm{t}+\Delta \mathrm{t}}^{\mathrm{e}}-\mathbf{I}\right]
$$

The hyperelastic constitutive law is used to calculate the $2^{\text {nd }}$ Piola-Kirchhoff stress in the intermediate configuration via

$$
\hat{\boldsymbol{\sigma}}_{\mathrm{t}+\Delta \mathrm{t}}^{\mathrm{PK2}}=\hat{\boldsymbol{L}}: \hat{\mathbf{E}}_{\mathrm{t}+\Delta \mathrm{t}}^{\mathrm{e}}
$$

Finally, the Cauchy stress at the end of the time step in the current configuration was determined by

$$
\sigma_{\mathrm{t}+\Delta t}=\frac{1}{J^{\mathbf{F}^{\mathrm{t}}+\Delta t}}\left[\mathbf{F}_{\mathrm{t}+\Delta \mathrm{t}}^{\mathrm{e}} \cdot \hat{\boldsymbol{\sigma}}_{\mathrm{t}+\Delta \mathrm{t}}^{\mathbf{P K} 2} \cdot\left(\mathbf{F}_{\mathrm{t}+\Delta \mathrm{t}}^{\mathrm{e}}\right)^{\mathrm{T}}\right]
$$

The final part of the stress update algorithm involves updating the current lattice orientations. $\mathbf{F}_{\mathrm{t}+\Delta t}^{\mathrm{e}}$ is separated into $\mathbf{U}_{\mathrm{t}+\Delta \mathrm{t}}^{\mathrm{e}}$ and $\mathbf{R}_{\mathrm{t}+\Delta \mathrm{t}}^{\mathbf{e}}$ according to the right polar decomposition. The updated lattice orientations are calculated via

$$
\mathbf{R}_{\mathrm{t}+\Delta \mathrm{t}}^{\mathrm{Lat}}=\mathbf{R}_{\mathrm{t}+\Delta \mathrm{t}}^{\mathrm{e}} \cdot \mathbf{R}_{\mathrm{h}}^{\mathrm{e}}
$$

where $\mathbf{R}^{\mathbf{L a t}}$ is the updated or current lattice orientation. 


\subsubsection{Hardening}

The hardening routine updates $\tau^{\mathrm{CSS}-\alpha}$ via Taylor's equation. In the local model, the total dislocation density, $\langle\rho\rangle$, is composed only of $\rho^{\text {SSD }}$, whose evolution was governed by the Kocks-Mecking equation (Eq. 3.69). Since the Kocks-Mecking equation depends on the amount of slip accumulated over $\Delta \mathrm{t}\left(\mathrm{d} \gamma^{\alpha}\right)$, the first step in the hardening routine is to integrate $\dot{\gamma}_{\mathrm{t}}^{\alpha}$ over $\Delta \mathrm{t}$ with a forward Euler approach

$$
\mathrm{d} \gamma^{\alpha}=\left|\dot{\gamma}_{\mathrm{t}}^{\alpha}\right| \Delta \mathrm{t}
$$

$\rho^{\text {SSD }}$ is then updated by integrating the Kocks-Mecking equation

$$
\rho_{t+\Delta t}^{S S D}=\rho_{t}^{S S D-\alpha}+\left[c_{K M 1} \sqrt{\rho_{t}^{S S D-\alpha}}-c_{K M 2} \rho_{t}^{S S D-\alpha}\right] d \gamma^{\alpha}
$$

Once $\rho_{t+\Delta t}^{\text {SSD- } \alpha}$ is determined, $\tau_{t+\Delta t}^{\text {CSS- } \alpha}$ is calculated with Taylor's equation, i.e.

$$
\tau_{t+\Delta t}^{\mathrm{CSS}-\alpha}=\mathrm{CG}_{110} \mathrm{~b} \sqrt{\rho_{\mathrm{t}+\Delta \mathrm{t}}^{\mathrm{SSD}-\alpha}}
$$

Table 4.3 contains a summary of the important equations used in the local model. 
Table 4.2 - Summary of the Local Crystal Plasticity Model.

\begin{tabular}{|c|c|}
\hline 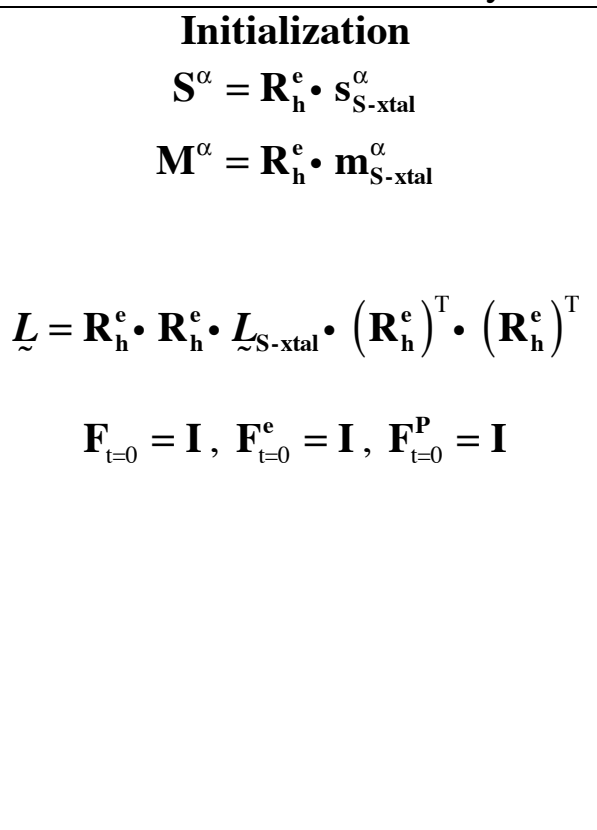 & 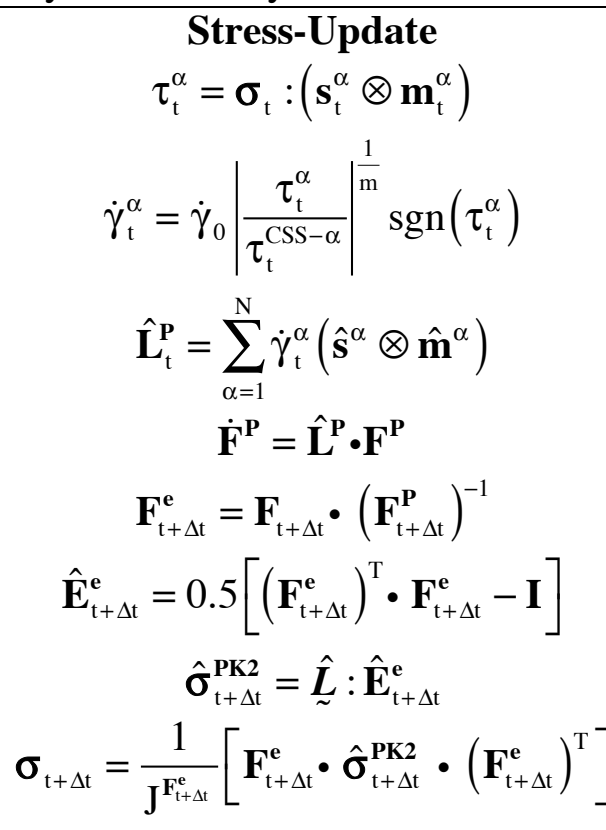 \\
\hline $\begin{array}{r}\text { Har } \\
\rho_{\mathrm{t}+\Delta \mathrm{t}}^{\mathrm{SSD}-\alpha}=\rho_{\mathrm{t}}^{\mathrm{SSD}-\alpha}+\left[\mathrm{c}_{\mathrm{KM} 1}\right. \\
\tau_{\mathrm{t}+\Delta \mathrm{t}}^{\mathrm{CSS}-\alpha}=\mathrm{C}\end{array}$ & $\left.-\frac{c_{\mathrm{KM} 2}}{\mathrm{SSD}-\alpha_{\mathrm{t}+\Delta \mathrm{t}}} \rho_{\mathrm{t}}^{\mathrm{SSD}-\alpha}\right] \mathrm{d} \gamma^{\alpha}$ \\
\hline
\end{tabular}




\subsubsection{Stability Check/Sub-incrementation}

Numerical instabilities arise in the stress update portion of the algorithm for 2 reasons: 1) the stiff power law viscoplastic flow rule and 2) the explicit integration scheme. These instabilities necessitate the need for a stability check to prevent

oscillations in $\sigma_{t+\Delta t}$. Once $\sigma_{t+\Delta t}$ and $\tau_{t+\Delta t}^{\text {CSS }-\alpha}$ are calculated, the stability of these values are checked. If it appears that $\sigma_{t+\Delta t}$ is unstable, then the total time step is broken into smaller steps (otherwise known as sub-increments) and the stress update and hardening parts of the model are repeated with these smaller time steps.

Observations of instabilities in the stress update algorithm revealed that stability could be guaranteed as long as $\left(\dot{\gamma}_{t+\Delta t}^{\alpha}-\dot{\gamma}_{t}^{\alpha}\right)$ is not large. Therefore, the $1^{\text {st }}$ step in the stability check is to re-run a small part of the update stress algorithm with values at $t+\Delta t$ to determine $\dot{\gamma}_{t+\Delta t}^{\alpha}$. To this end, the stability check starts by projecting the Cauchy stress at time $t+\Delta t$ onto each of the slip systems in the current configuration by

$$
\tau_{t+\Delta t}^{\alpha}=\sigma_{t+\Delta t}:\left(\mathbf{s}_{t+\Delta t}^{\alpha} \otimes \mathbf{m}_{t+\Delta t}^{\alpha}\right)
$$

The shearing rates are then calculated using the same power law flow rule with updated information

$$
\dot{\gamma}_{t+\Delta t}^{\alpha}=\dot{\gamma}_{0}\left|\frac{\tau_{t+\Delta t}^{\alpha}}{\tau_{t+\Delta t}^{C S S-\alpha}}\right|^{\frac{1}{m}} \operatorname{sgn}\left(\tau_{t+\Delta t}^{\alpha}\right) .
$$

From this information, the quantity, $\left\|\dot{\gamma}_{t+\Delta t}^{\alpha}\right\|$, is defined as

$$
\left\|\dot{\gamma}_{t+\Delta t}^{\alpha}\right\|=\sqrt{\left(\dot{\gamma}_{t+\Delta t}^{\alpha}-\dot{\gamma}_{t}^{\alpha}\right)^{2}+(\Delta t)^{2}}
$$


Instabilities in $\sigma_{\mathrm{t}+\Delta \mathrm{t}}$ appear when $\Delta \dot{\gamma}^{\alpha}$ is too large. So when $\Delta \dot{\gamma}^{\alpha}$ is large, a small $\Delta \mathrm{t}$ is needed to ensure stability, but when $\Delta \dot{\gamma}^{\alpha}$ is small a larger $\Delta \mathrm{t}$ was possible.

Since a single critical value of $\left\|\dot{\gamma}_{\mathrm{t}+\Delta t}^{\alpha}\right\|\left(\left\|\dot{\gamma}_{\mathrm{t}+\Delta t}^{\alpha}\right\|^{\text {CRIT }}\right)$ does not guarantee stability for all $\Delta \mathrm{t}$, a $\left\|\dot{\gamma}_{t+\Delta t}^{\alpha}\right\|^{\text {CRIT }}$ for various time steps, as shown in Table 4.4, was determined.

Table $4.3-\left\|\dot{\gamma}_{t+\Delta t}^{\alpha}\right\|^{\text {CRIT }}$ needed for stability in the crystal plasticity models

\begin{tabular}{|c|c|}
\hline$\Delta \mathrm{t}$ & $\left\|\dot{\gamma}_{\mathrm{t}+\Delta \mathrm{t}}^{\alpha}\right\|^{\text {CRIT }}$ \\
\hline$\Delta \mathrm{t} \geq 1 \times 10^{-3}$ & $100^{*} \Delta \mathrm{t}$ \\
\hline $1 \times 10^{-3}<\Delta \mathrm{t} \leq 2 \times 10^{-4}$ & $10^{*} \Delta \mathrm{t}$ \\
\hline $2 \times 10^{-4}<\Delta \mathrm{t} \leq 1 \times 10^{-4}$ & 0.003 \\
\hline $1 \times 10^{-4}<\Delta \mathrm{t} \leq 1 \times 10^{-5}$ & 0.05 \\
\hline $1 \times 10^{-5}<\Delta \mathrm{t} \leq 1 \times 10^{-6}$ & 0.0075 \\
\hline $1 \times 10^{-6}<\Delta \mathrm{t} \leq 1 \times 10^{-7}$ & 0.025 \\
\hline $1 \times 10^{-7}<\Delta \mathrm{t} \leq 1 \times 10^{-8}$ & 0.02 \\
\hline $1 \times 10^{-8}<\Delta \mathrm{t} \leq 1 \times 10^{-9}$ & 0.2 \\
\hline$\Delta \mathrm{t}<1 \times 10^{-9}$ & 50.0 \\
\hline
\end{tabular}

If $\left\|\dot{\gamma}_{\mathrm{t}+\Delta t}^{\alpha}\right\|<\left\|\dot{\gamma}_{\mathrm{t}+\Delta t}^{\alpha}\right\|^{\text {CRIT }}$, then $\sigma_{\mathrm{t}+\Delta \mathrm{t}}$ is considered stable. The crystal plasticity model updates the state variables and returns $\sigma_{t+\Delta t}$ to the FEM code. If, however, $\left\|\dot{\gamma}_{t+\Delta t}^{\alpha}\right\|>$ $\left\|\dot{\gamma}_{t+\Delta t}^{\alpha}\right\|^{\text {CRIT }}$, then $\sigma_{t+\Delta t}$ is deemed unstable and the crystal plasticity model sub-increments $\Delta \mathrm{t}$.

Sub-incrementation starts by dividing the unstable time step in half and redoing the update stress and hardening algorithms in two steps. If one of the two sub-increments is also unstable, then this time step is divided in two. This process continues until the model reaches time $t+\Delta t$ with stable time steps, as illustrated in Figure 4.2. 


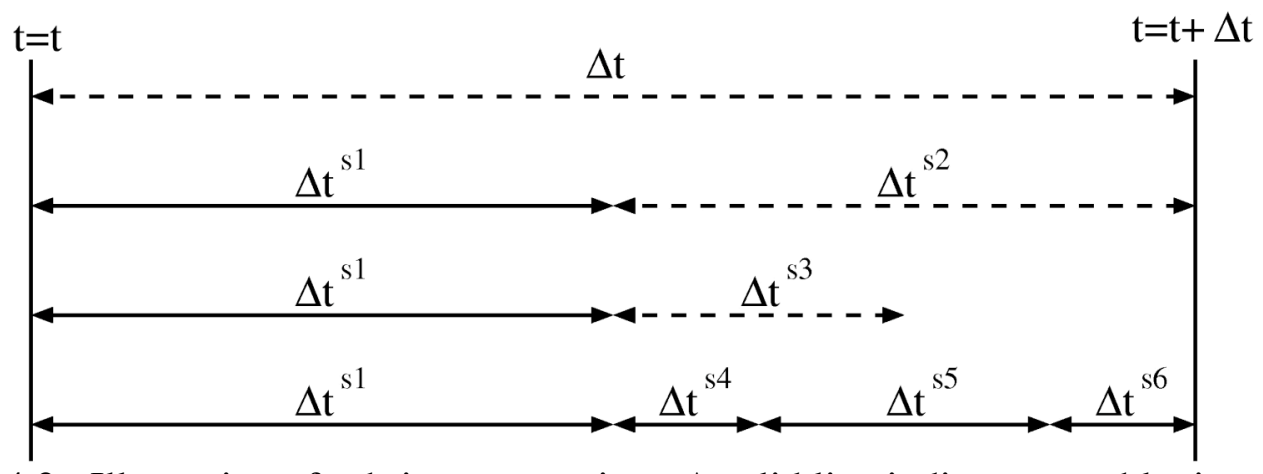

Figure 4.2 - Illustration of sub-incrementation. A solid line indicates a stable time step, and a dashed line represents an unstable time step.

In Figure 4.2, the initial time step $\Delta \mathrm{t}$ is found to be unstable. So $\Delta \mathrm{t}$ is subdivided into 2 smaller time steps $\Delta \mathrm{t}^{\mathrm{s} 1}$ and $\Delta \mathrm{t}^{\mathrm{s} 2} . \Delta \mathrm{t}^{\mathrm{s} 1}$ is found to be stable however $\Delta \mathrm{t}^{\mathrm{s} 2}$ is not. $\Delta \mathrm{t}^{\mathrm{s} 2}$ is then cut in half to $\Delta \mathrm{t}^{\mathrm{s} 3}$, but this time step is also unstable. Thus, $\Delta \mathrm{t}^{\mathrm{s} 3}$ is cut in half resulting in a stable time step $\Delta \mathrm{t}^{\mathrm{s} 4}$. Anytime a sub-increment time step is stable (like $\Delta \mathrm{t}^{\mathrm{s} 1}$ and $\Delta \mathrm{t}^{\mathrm{s}}$ in Figure 4.2), the sub-incrementation algorithm attempts to accelerate the time stepping. The accelerated time step is determined by doubling previously stable time step as long this new time step does not result in a total time step larger than $\mathrm{t}+\Delta \mathrm{t}$. In Figure $4.2, \Delta \mathrm{t}^{\mathrm{s} 5}$ is twice as large as $\Delta \mathrm{t}^{\mathrm{s} 4}$, but $2 * \Delta \mathrm{t}^{\mathrm{s} 5}$ and $2 * \Delta \mathrm{t}^{\mathrm{s} 2}$ results in a total time step larger than $\mathrm{t}+\Delta \mathrm{t}$. $\Delta \mathrm{t}^{\mathrm{s} 5}$ and $\Delta \mathrm{t}^{\mathrm{s} 2}$ are then simply the amount of time left in the total time step $\Delta \mathrm{t}$. A subtlety imbedded in the sub-incrementation approach is that $\mathbf{F}$ is only known at $t$ and $t+\Delta t$. By assuming that the velocity gradient is constant over $\Delta \mathrm{t}$, it was possible to linearly interpolate $\mathbf{F}$ between $\mathrm{t}$ and $\mathrm{t}+\Delta \mathrm{t}$ during sub-incrementation. 


\subsubsection{Integration Scheme Convergence}

The convergence of the integration scheme was also checked by simulating a single cryastl with different mesh densities. Results of the convergence test are shown in

Figure 4.3.

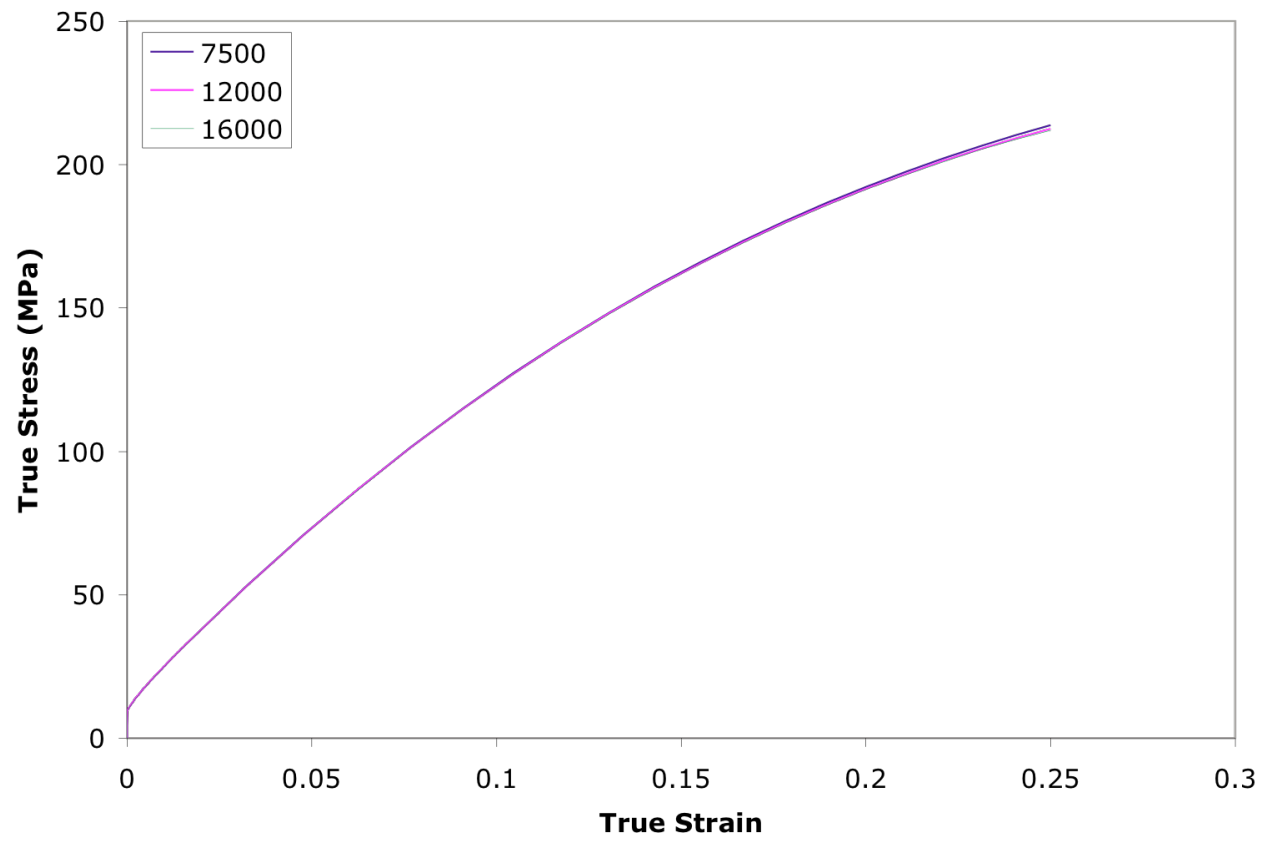

Figure 4.3 - Stress-strain response of a single crystal meshed with between 7500 and 16,000 elements.

Changing the number of elements from 7,500 to 16,000 did not change the macro-scopic stress-strain response. Thus, the forward Euler integration scheme is accurate and does converge. 


\subsection{Non-Local Crystal Plasticity Model}

The major conceptual difference between the local and non-local models is the composition of $\langle\rho\rangle$. In the local model $\langle\rho\rangle$, is comprised of $\rho^{\text {SSD }}$ only because the local model is applied to materials where $\rho^{\mathrm{SSD}}>>\rho^{\mathrm{GND}}$. In the non-local model, $\langle\rho\rangle$ is made up of $\rho^{\mathrm{SSD}}+\rho^{\mathrm{GND}}$, because the non-local model is applied to materials with an appreciable $\rho^{\mathrm{GND}}$

The state of $\rho^{\mathrm{GND}}$ in the material is governed by the polycrystal $\mathbf{F}^{\mathrm{e}} \mathbf{F}^{\mathrm{p}}$ kinematics. $\rho^{\mathrm{GND}}$ is derived from the total GND tensor $\left(\hat{\mathbf{G}}^{\mathrm{Tot}}\right)$, which in turn depends on the sum of the natural configuration GND tensor $(\tilde{\mathbf{G}})$ and the intermediate configuration GND tensor $(\hat{\mathbf{G}})$ according to Eq. 3.32. The gradient part of both $\tilde{\mathbf{G}}$ and $\hat{\mathbf{G}}$ is calculated using the integral approximation technique. Thus the gradient based dislocation tensors, $\tilde{\mathbf{G}}$ and $\hat{\mathbf{G}}$, are approximated using the following non-local integral based dislocation tensors:

$$
\begin{aligned}
& \{\tilde{\mathbf{G}}\}=\mathbf{R}_{\mathbf{h}}^{\mathbf{e}^{-1}} \cdot\left\{\operatorname{Curl} \mathbf{R}_{\mathbf{h}}^{\mathbf{e}^{-1}}\right\} \\
& \{\hat{\mathbf{G}}\}=\frac{1}{\mathbf{J}^{\mathbf{F}^{\mathbf{p}}}} \mathbf{F}^{\mathbf{p}} \cdot\left\{\operatorname{Curl} \mathbf{F}^{\mathbf{p}}\right\}
\end{aligned}
$$

where $\{\operatorname{Curl} \mathbf{H}\}_{\mathrm{ni}} \equiv e_{\mathrm{nkj}}\{\nabla \mathbf{H}\}_{\mathrm{ij}, \mathrm{k}}$. Thermodynamically, the use of non-local integral based dislocation tensors means that higher order strain gradients are not present in the free energy functional. The free energy functional for this model is $\psi=\bar{\psi}\left(\hat{\mathbf{E}}^{\mathrm{e}}\left(\mathbf{F}^{\mathrm{e}}\right)\right)$. There are no higher order quantities associated with $\{\tilde{\mathbf{G}}\}$ because $\mathbf{R}_{\mathbf{h}}^{\mathbf{e}}$ is constant. There are no 
higher order quantities associated with $\{\hat{\mathbf{G}}\}$ due to the following constitute assumption: $\psi$ does not depend on $\left\{\operatorname{Curl} \mathbf{F}^{\mathbf{P}}\right\}$. Thus, there is no need to account for higher order stresses in the constitutive relations.

The non-local integral based tensors $\{\hat{\mathbf{G}}\}$ and $\{\tilde{\mathbf{G}}\}$ are better suited to characterize grain boundaries than dislocation tensors based solely on local incompatibility, $\hat{\mathbf{G}}$ and $\tilde{\mathbf{G}}$, both physically and computationally. Physically, the exponential function is appropriate because it mimics a $\frac{1}{\mathrm{r}}$ dependence, similar to stress fields associated with dislocation-dislocation and dislocation-grain boundary interactions that determine hardening. Thus $\{\hat{\mathbf{G}}\}$ and $\{\tilde{\mathbf{G}}\}$ better capture the longer range, i.e. inherently "non-local", stress fields associated with microstructure level interactions. Computationally, $\{\hat{\mathbf{G}}\} \approx \hat{\mathbf{G}}$ and $\{\tilde{\mathbf{G}}\} \approx \tilde{\mathbf{G}}$ when the tensor fields $\mathbf{F}^{\mathbf{P}}$ and $\mathbf{R}_{\mathbf{e}}^{\mathbf{h}}$ are homogeneous (i.e. throughout the grain interior). This is an important point since local incompatibility based dislocation tensors were rigorously derived for single crystals where all the tensor fields vary continuously. However, there is a singularity at the discontinuity in the $\mathbf{F}^{\mathbf{P}}$ and $\mathbf{R}_{\mathrm{e}}^{\mathbf{h}}$ fields (i.e., at grain boundaries) for $\hat{\mathbf{G}}$ and $\tilde{\mathbf{G}}$. This singularity is numerically resolved with interpolation (or shape) functions in FEM, which have no physical meaning. With the non-local integral approach, there is no singularity at the grain boundary.

The numerical algorithm used in the non-local crystal plasticity model can be broken into the same 4 parts as the local model: 1) initialization, 2) stress update, 3) hardening, and 4) stability check/sub-incrementation. Because the local crystal plasticity 
model was used as the foundation for the non-local model, many of the local model's features are re-used in the non-local model. Specifically, the stress update and stability/sub-incrementation algorithms are unchanged between the two models. Thus a description of only the initialization and hardening routines follows

\subsubsection{Initialization}

The initialization routine for the non-local model initializes the crystal structure in the same manner that the local model's initialization routine does: $\mathbf{S}^{\alpha}=\mathbf{R}_{\mathbf{h}}^{\mathrm{e}} \cdot \mathbf{S}_{\mathrm{S} \text {-xtal }}^{\alpha}$, $\mathbf{M}^{\alpha}=\mathbf{R}_{\mathbf{h}}^{\mathrm{e}} \bullet \mathbf{m}_{\text {S-xtal }}^{\alpha}$, and $\underset{\sim}{\boldsymbol{L}}=\mathbf{R}_{\mathrm{h}}^{\mathrm{e}} \bullet \mathbf{R}_{\mathrm{h}}^{\mathrm{e}} \cdot \underset{\sim \mathrm{S}-\mathrm{xtal}}{\boldsymbol{L}} \bullet\left(\mathbf{R}_{\mathbf{h}}^{\mathrm{e}}\right)^{\mathrm{T}} \bullet\left(\mathbf{R}_{\mathrm{h}}^{\mathrm{e}}\right)^{\mathrm{T}}$. The reference configuration is also assumed to be stress free making $\mathbf{F}_{\mathrm{t}=0}=\mathbf{I}, \mathbf{F}_{\mathrm{t}=0}^{\mathbf{e}}=\mathbf{I}, \mathbf{F}_{\mathrm{t}=0}^{\mathbf{P}}=\mathbf{I}$.

In the non-local model, the initial hardening state (represented by $\tau_{\mathrm{t}=0}^{\mathrm{CSS}-\alpha}$ ) cannot be determined from the input parameters alone. Rather, $\{\tilde{\mathbf{G}}\}$ has to first be calculated. The $1^{\text {st }}$ step in this calculation is to define the integration volume for each element by creating a neighbor list. The neighbor list contains all the surrounding elements over which the non-local integrals associated with $\{\tilde{\mathbf{G}}\}$ are determined. This same neighbor list is used for $\{\hat{\mathbf{G}}\}$. The neighbor lists are built using a distance criterion. If the distance between two element centroids is less than a user defined radius, then each element is added to the other's neighbor list.

This neighbor cloud radius is usually much smaller than the material body even though the non-local integrals associated with $\{\tilde{\mathbf{G}}\}$ and $\{\hat{\mathbf{G}}\}$ are defined over the entire 
material body. The exponential attenuation functions in $\{\tilde{\mathbf{G}}\}$ and $\{\hat{\mathbf{G}}\}$ approach zero but never reach zero meaning that every point in the material body affects every other point in the body. The attenuation function parameter, $\omega$, can be chosen so that elements far away make a negligible contribution to $\{\tilde{\mathbf{G}}\}$ and $\{\hat{\mathbf{G}}\}$. Thus it is computationally advantageous to minimize the neighbor cloud radius for a given $\omega$. This minimized neighbor cloud is determined by observing the change in $\tau^{\mathrm{CSS}-\alpha}$ as a large neighbor cloud was reduced. A neighbor cloud is considered minimized when the change in $\tau^{\mathrm{CSS}-\alpha}$ is greater than $0.01 \%$.

Once the neighbor lists are created, any lattice symmetries that exists in the material is enforced. Materials with cubic symmetry, like fcc materials, have 24 orientation tensors that represent equivalent crystal orientations ${ }^{8}$. Before calculating $\{\tilde{\mathbf{G}}\}$, the misorientations between $\mathbf{R}_{\mathbf{h}}^{\mathrm{e}}$ at one point and the 24 symmetrically equivalent values of $\mathbf{R}_{\mathbf{h}}^{\mathrm{e}}$ from the other point are determined. The one symmetrically equivalent value of $\mathbf{R}_{\mathbf{h}}^{\mathbf{e}}$ that returns the lowest misorientation is used in the $\{\tilde{\mathbf{G}}\}$ calculation.

The symmetry check starts by finding the orientation $(\mathbf{j})$ needed to bring the crystal lattice at a point $\mathbf{x}+\xi$ into coincidence with the crystal lattice at point $\mathbf{x}$

$$
\mathbf{j}=\mathbf{R}_{\mathbf{h}}^{\mathrm{e}}(\mathbf{x}) \cdot\left[\mathbf{R}_{\mathbf{h}}^{\mathrm{e}}(\mathbf{x}+\xi)\right]^{\mathrm{T}}
$$

The misorientation angle, $\theta$, is then be found by

$$
\theta=\cos ^{-1}\left(0.5 *\left[\mathrm{j}_{11}+\mathrm{j}_{22}+\mathrm{j}_{33}-1\right]\right) \text {. }
$$

\footnotetext{
${ }^{8}$ The 24 symmetric orientation tensors are listed in Appendix A.
} 
This procedure is done for each of the 24 symmetric orientation tensors associated with $\mathbf{R}_{\mathbf{h}}^{\mathrm{e}}(\mathbf{x}+\xi)$. The orientation tensor that yielded the smallest $\theta$ is taken as the symmetrically equivalent orientation, $\mathbf{R}^{\text {sym }}(\mathbf{x}+\xi)^{9}$.

The non-local integral equation for $\{\tilde{\mathbf{G}}\}$ with $\mathbf{R}^{\text {sym }}(\mathbf{x}+\xi)$ becomes

$$
\{\tilde{\mathbf{G}}\}=\left(\int_{V_{\text {cell }}}\left(\mathbf{R}^{\mathrm{sym}^{-1}}(\mathbf{x}+\xi)-\mathbf{R}_{\mathbf{h}}^{\mathrm{e}^{-1}}(\mathbf{x})\right) \otimes \xi \Theta(\mathbf{r}) \mathrm{d} \xi\right) \cdot\left(\int_{\mathrm{Vcell}} \xi \otimes \xi \Theta(\mathbf{r}) \mathrm{d} \xi\right)^{-1}
$$

In the finite element setting where space is discretized, these non-local integrals can be expressed as summations over all the elements in the neighbor list (Stromberg and Ristinmaa, 1996). The integral in the numerator is a $3^{\text {rd }}$ order tensor $\left(A_{i j m}\right)$ calculated by

$$
\mathrm{A}_{\mathrm{ijm}}=\sum_{*=1}^{\# \mathrm{~N}}\left[\left(\mathrm{R}^{\mathrm{sym} \mathrm{m}^{*}}\right)_{\mathrm{ij}}^{-1}-\left(\mathrm{R}_{\mathrm{h}}^{\mathrm{e}}\right)_{\mathrm{ij}}^{-1}\right] \xi_{\mathrm{m}} \Theta(\mathbf{r}) \mathrm{vol}^{*}
$$

where \#N is the number of neighbors, $\left(\mathrm{R}^{\mathrm{sym} *}\right)_{\mathrm{ij}}$ is the symmetric orientation tensor for neighboring element $*, \xi_{\mathrm{m}}$ is the distance between element $*$ and the element at $\mathbf{x}$ in the m direction, and vol* is the volume of neighboring element $*$. The integral in the denominator is a $2^{\text {nd }}$ order tensor $\left(B_{\mathrm{km}}\right)$ that was determined via

$$
\mathrm{B}_{\mathrm{km}}=\sum_{\mathrm{n}=1}^{\# \mathrm{~N}} \xi_{\mathrm{k}} \xi_{\mathrm{m}} \Theta(\mathbf{r}) \mathrm{vol}^{\mathrm{n}}
$$

$\left\{\operatorname{Curl} \mathbf{R}_{\mathbf{h}}^{\mathbf{e}^{-1}}\right\}$ is computed with the matrix equation

$$
\left\{\operatorname{Curl} \mathbf{R}_{\mathrm{h}}^{\mathrm{e}^{-1}}\right\}_{\mathrm{ni}}=e_{\mathrm{nkj}} \mathrm{A}_{\mathrm{ijm}}\left(\mathrm{B}_{\mathrm{km}}\right)^{-1}
$$

and $\{\tilde{\mathbf{G}}\}$ is calculated as

${ }^{9} \mathbf{R}^{\text {sym }}(\mathbf{x}+\xi)$ is only used during the calculation of $\{\tilde{\mathbf{G}}\}$. 


$$
\{\tilde{\mathbf{G}}\}=\mathbf{R}_{\mathbf{h}}^{\mathbf{e}^{-1}} \cdot\left\{\operatorname{Curl} \mathbf{R}_{\mathbf{h}}^{\mathbf{e}^{-1}}\right\}
$$

Finally, $\{\tilde{\mathbf{G}}\}$ is pushed forward to the intermediate configuration where it initializes $\hat{\mathbf{G}}^{\text {Tot }}$ via

$$
\hat{\mathbf{G}}_{\mathrm{t}=0}^{\mathrm{Tot}}=\mathbf{R}_{\mathbf{h}}^{\mathbf{e}}(\mathbf{x}) \cdot\{\tilde{\mathbf{G}}\} \cdot\left[\mathbf{R}_{\mathbf{h}}^{\mathbf{e}}(\mathbf{x})\right]^{\mathrm{T}}
$$

Since Taylor's hardening equation accounts for the effects of a scalar dislocation density, $\hat{\mathbf{G}}_{\mathrm{t}=0}^{\text {Tot }}$ is converted to a scalar $\hat{\rho}_{\mathrm{t}=0}^{\mathrm{GND}}$. Since there is no well-accepted way to do this (See Chapter 3.3.2.2), a computationally efficient method with some physical meaning is employed: a projection of $\hat{\mathbf{G}}_{\mathrm{t}=0}^{\mathrm{Tot}}$ onto the Schmid tensor

$$
\hat{\rho}_{\mathrm{t}=0}^{\mathrm{GND}-\alpha}=\frac{\left|\hat{\mathbf{G}}_{\mathrm{t}=0}^{\mathrm{Tot}}:\left(\hat{\mathbf{s}}^{\alpha} \otimes \hat{\mathbf{m}}^{\alpha}\right)\right|}{\mathrm{b}} .
$$

Because no distinction is made between positively and negatively signed dislocations, the absolute value in Eq. (4.35) ensured that dislocations of both signs contributed to the overall density. The initial GND density in the intermediate configuration is pushed forward to the current configuration via

$$
\rho_{\mathrm{t}=0}^{\mathrm{GND}}=\mathrm{J}^{\mathbf{F}_{\mathrm{t}=0}^{\mathrm{e}}} \hat{\rho}_{\mathrm{t}=0}^{\mathrm{GND}}
$$

where $\rho_{\mathrm{t}=0}^{\mathrm{GND}}$ is the initial GND density in the current configuration and $\mathbf{J}^{\mathrm{F}_{t=0}^{\mathrm{e}}}=1$. Physically, $\rho_{\mathrm{t}=0}^{\mathrm{GND}}$ and $\hat{\rho}_{\mathrm{t}=0}^{\mathrm{GND}}$ represent the effect of GNDs in dislocation arrays (like grain boundaries) initially present in the material.

The initial SSD density $\left(\rho_{\mathrm{t}=0}^{\mathrm{SSD}-\alpha}\right)$ in the non-local model is found by considering the SSD state of a material where $\rho^{\mathrm{SSD}}>>\rho^{\mathrm{GND}}$. Such a criterion ensures that when SSDs dominate GDNs, the non-local model yields the same result as the local model. The 
hardening state of a SSD dominated material was represented by the hardening input parameter $\tau_{\mathrm{t}=0}^{\mathrm{LIM}} \cdot \rho_{\mathrm{t}=0}^{\mathrm{SSD}-\alpha}$ is then found by inverting Taylor's equation with $\langle\rho\rangle=\rho^{\text {SSD }}$

$$
\rho_{\mathrm{t}=0}^{\mathrm{SSD}-\alpha}=\left(\frac{\tau_{\mathrm{t}=0}^{\mathrm{LIM}}}{\mathrm{CG}_{110} \mathrm{~b}}\right)^{2} .
$$

For simplicity, it is assumed that $\tau_{\mathrm{t}=0}^{\mathrm{LIM}}$, and therefore $\rho_{\mathrm{t}=0}^{\mathrm{SSD}-\alpha}$, is equivalent on each slip system.

Once $\rho_{\mathrm{t}=0}^{\mathrm{GND}-\alpha}$ and $\rho_{\mathrm{t}=0}^{\mathrm{SSD}-\alpha}$ are determined, $\tau_{\mathrm{t}=0}^{\mathrm{CSS}-\alpha}$ is calculated using Taylor's equation

$$
\tau_{\mathrm{t}=0}^{\mathrm{CSS}-\alpha}=\mathrm{CG}_{110} \mathrm{~b} \sqrt{\rho_{\mathrm{t}=0}^{\mathrm{SSD}-\alpha}+\rho_{\mathrm{t}=0}^{\mathrm{GND}-\alpha}} .
$$

Even though $\rho_{\mathrm{t}=0}^{\text {SSD- } \alpha}$ is the same on each slip system, the initial hardness of each slip system is not constant because $\rho_{\mathrm{t}=0}^{\mathrm{GND}-\alpha}$ is not necessarily the same on each slip system.

\subsubsection{Hardening}

Similar to the local model, $\tau_{\mathrm{t}+\Delta \mathrm{t}}^{\mathrm{CSS} \alpha}$ is the critical state variable updated by the hardening routine in the non-local model. However, in the non-local model $\tau_{t+\Delta t}^{\text {CSS- } \alpha}$ is a function of both $\rho_{t+\Delta t}^{\text {SSD } \alpha}$ and $\rho_{t+\Delta t}^{\text {GND }}$. Because the evolution of $\rho_{t+\Delta t}^{\text {SSD- } \alpha}$ depends on $\rho_{t}^{\text {GND }}$ and not on $\rho_{t+\Delta t}^{\mathrm{GND}}$, each dislocation density is calculated separately. $\rho_{\mathrm{t}+\Delta \mathrm{t}}^{\mathrm{SSD}-\alpha}$ evolves according to a Kocks-Mecking evolution equation

$$
\rho_{t+\Delta t}^{\mathrm{SSD}-\alpha}=\rho_{\mathrm{t}}^{\mathrm{SSD}-\alpha}+\left[\mathrm{c}_{\mathrm{KM} 1} \sqrt{\rho_{\mathrm{t}}^{\mathrm{SSD}-\alpha}+\rho_{\mathrm{t}}^{\mathrm{GND}-\alpha}}-c_{\mathrm{KM} 2}\left(\rho_{\mathrm{t}}^{\mathrm{SSD}-\alpha}+\rho_{\mathrm{t}}^{\mathrm{GND}-\alpha}\right)\right] \mathrm{d} \gamma^{\alpha}
$$

where $\mathrm{d} \gamma^{\alpha}$ is determined by integrating $\dot{\gamma}^{\alpha}$ over $\Delta \mathrm{t}$ in a forward Euler manner (Eq. 4.18). 
The $\rho^{\mathrm{GND}}$ update algorithm is based on the integral equation for $\{\hat{\mathbf{G}}\}$

$$
\{\hat{\mathbf{G}}\}=\left(\int_{\mathrm{Vcell}}\left(\mathbf{F}^{\mathbf{P}}(\mathbf{x}+\xi)-\mathbf{F}^{\mathbf{P}}(\mathbf{x})\right) \otimes \xi \Theta(\mathbf{r}) \mathrm{d} \xi\right) \cdot\left(\int_{\mathrm{V} \text { cell }} \xi \otimes \xi \alpha(\mathbf{r}) \mathrm{d} \xi\right)^{-1}
$$

The integral in the numerator is transformed to a summation equation and solved by

$$
\mathrm{A}_{\mathrm{ijm}}=\sum_{*=1}^{\# \mathrm{~N}}\left[\left(\mathrm{~F}^{\mathrm{P}}\right)_{\mathrm{ij}}-\left(\mathrm{F}^{\mathrm{P}}\right)_{\mathrm{ij}}\right] \xi_{\mathrm{m}} \Theta(\mathbf{r}) \mathrm{vol}^{*}
$$

where $\mathrm{F}^{\mathrm{P} *}$ is $\mathrm{F}^{\mathrm{P}}$ for neighboring element $*$ and vol* is the volume of element *. The summation equation for the integral in the denominator is

$$
\mathrm{B}_{\mathrm{km}}=\sum_{*=1}^{\# \mathrm{~N}} \xi_{\mathrm{k}} \xi_{\mathrm{m}} \Theta(\mathbf{r}) \mathrm{vol}^{*}
$$

$\left\{\operatorname{Curl} \mathbf{F}^{\mathbf{P}}\right\}$ is then computed via the matrix equation

$$
\left\{\operatorname{Curl} \mathbf{F}^{\mathbf{P}}\right\}_{\mathrm{ni}}=e_{\mathrm{nkj}} \mathrm{A}_{\mathrm{ijm}}\left(\mathrm{B}_{\mathrm{km}}\right)^{-1}
$$

and $\{\hat{\mathbf{G}}\}$ is calculated as

$$
\{\hat{\mathbf{G}}\}=\mathbf{F}^{\mathbf{P}} \cdot\left\{\operatorname{Curl} \mathbf{F}^{\mathbf{P}}\right\}
$$

$\hat{\mathbf{G}}_{\mathrm{t}+\Delta \mathrm{t}}^{\text {Tot }}$ is determined by adding $\{\hat{\mathbf{G}}\}$ and $\{\tilde{\mathbf{G}}\}$ pushed forward from the natural configuration to the intermediate configuration

$$
\hat{\mathbf{G}}_{\mathrm{t}=t+\Delta t}^{\mathrm{Tot}}=\mathbf{F}^{\mathbf{P}}(\mathbf{x}) \cdot \mathbf{R}_{\mathbf{h}}^{\mathbf{e}}(\mathbf{x}) \cdot\{\tilde{\mathbf{G}}\} \cdot\left[\mathbf{R}_{\mathbf{h}}^{\mathrm{e}}(\mathbf{x})\right]^{\mathrm{T}} \cdot \mathbf{F}^{\mathbf{P}^{-1}}(\mathbf{x})+\{\hat{\mathbf{G}}\} .
$$

Finally, $\hat{\rho}_{\mathrm{t}=t+\Delta \mathrm{t}}^{\mathrm{GND} \alpha}$ is determined by projecting $\hat{\mathbf{G}}_{\mathrm{t}=\mathrm{t}}^{\mathrm{Tot}}$ onto the Schmid tensor

$$
\hat{\rho}_{\mathrm{t}+\Delta \mathrm{t}}^{\mathrm{GND} \alpha}=\frac{\left|\hat{\mathbf{G}}_{\mathrm{t}+\Delta \mathrm{t}}^{\mathrm{Tot}}:\left(\mathbf{s}^{\alpha} \otimes \mathbf{m}^{\alpha}\right)\right|}{b} .
$$

$\hat{\rho}_{t+\Delta t}^{\mathrm{GND}-\alpha}$ is then pushed to the current configuration via Eq. (4.36), however in this case

$\mathbf{J}_{\mathrm{t}+\Delta \mathrm{t}}^{\mathbf{F}^{\mathrm{e}}} \neq 1 . \tau_{\mathrm{t}+\Delta \mathrm{t}}^{\mathrm{CSS} \alpha}$ is then calculated using Taylor's equation 


$$
\tau_{\mathrm{t}+\Delta \mathrm{t}}^{\mathrm{CSS}-\alpha}=\mathrm{CG}_{110} b \sqrt{\rho_{\mathrm{t}+\Delta \mathrm{t}}^{\mathrm{SSD}-\alpha}+\rho_{\mathrm{t}+\Delta \mathrm{t}}^{\mathrm{GND}-\alpha}}
$$

A summary of the non-local model is contained in Table 4.5.

Table 4.4 - Summary of the Non-Local Crystal Plasticity Model.

\begin{tabular}{|c|c|}
\hline 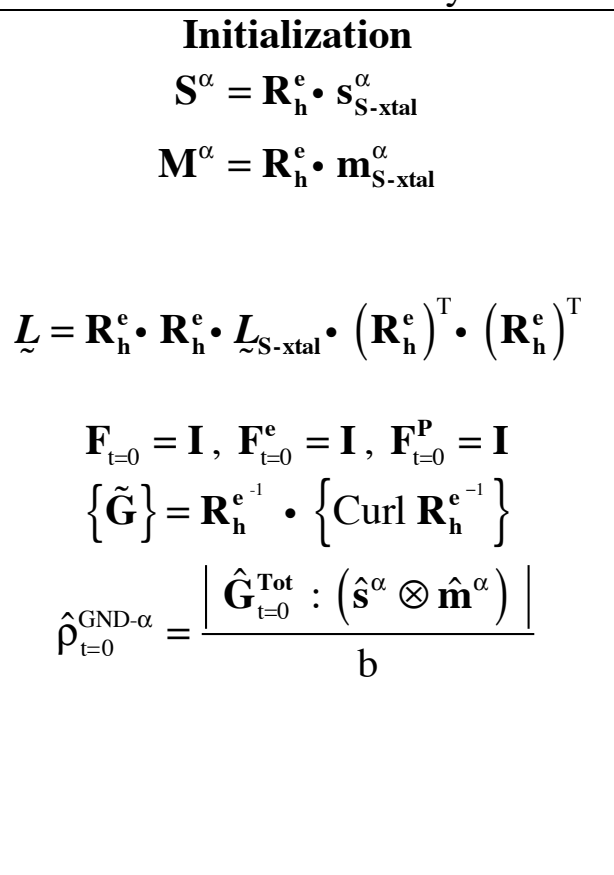 & $\begin{array}{c}\text { Stress-Update } \\
\tau_{\mathrm{t}}^{\alpha}=\sigma_{\mathrm{t}}:\left(\mathbf{s}_{\mathrm{t}}^{\alpha} \otimes \mathbf{m}_{\mathrm{t}}^{\alpha}\right) \\
\dot{\gamma}_{\mathrm{t}}^{\alpha}=\dot{\gamma}_{0}\left|\frac{\tau_{\mathrm{t}}^{\alpha}}{\tau_{\mathrm{t}}^{\mathrm{CSS}-\alpha}}\right|^{\frac{1}{\mathrm{~m}}} \operatorname{sgn}\left(\tau_{\mathrm{t}}^{\alpha}\right) \\
\hat{\mathbf{L}}_{\mathrm{t}}^{\mathbf{P}}=\sum_{\alpha=1}^{\mathrm{N}} \dot{\gamma}_{\mathrm{t}}^{\alpha}\left(\hat{\mathbf{s}}^{\alpha} \otimes \hat{\mathbf{m}}^{\alpha}\right) \\
\dot{\mathbf{F}}^{\mathbf{P}}=\hat{\mathbf{L}}^{\mathbf{P}} \cdot \mathbf{F}^{\mathbf{P}} \\
\mathbf{F}_{\mathrm{t}+\Delta \mathrm{t}}^{\mathbf{e}}=\mathbf{F}_{\mathrm{t}+\Delta \mathrm{t}} \cdot\left(\mathbf{F}_{\mathrm{t}+\Delta \mathrm{t}}^{\mathbf{P}}\right)^{-1} \\
\hat{\mathbf{E}}_{\mathrm{t}+\Delta \mathrm{t}}^{\mathrm{e}}=0.5\left[\left(\mathbf{F}_{\mathrm{t}+\Delta \mathrm{t}}^{\mathbf{e}}\right)^{\mathrm{T}} \cdot \mathbf{F}_{\mathrm{t}+\Delta \mathrm{t}}^{\mathbf{e}}-\mathbf{I}\right] \\
\boldsymbol{\sigma}_{\mathrm{t}+\Delta \mathrm{t}}=\frac{1}{\mathbf{J}^{\mathbf{F}_{\mathrm{t}+\Delta t}^{\mathrm{e}}}}\left[\mathbf{F}_{\mathrm{t}+\Delta \mathrm{t}}^{\mathbf{e}} \cdot \hat{\boldsymbol{\sigma}}_{\mathrm{t}+\Delta \mathrm{t}}^{\mathbf{P K} 2} \cdot\left(\mathbf{F}_{\mathrm{t}+\Delta \mathrm{t}}^{\mathrm{e}}\right)^{\mathrm{T}}\right]\end{array}$ \\
\hline \multicolumn{2}{|r|}{$\left.c_{\mathrm{KM} 2}\left(\rho_{\mathrm{t}}^{\mathrm{SSD}-\alpha}+\rho_{\mathrm{t}}^{\mathrm{GND}-\alpha}\right)\right] \mathrm{d} \gamma^{\alpha}$} \\
\hline
\end{tabular}




\section{CHAPTER 5}

\section{SENSITIVITY ANALYSIS OF THE NON-LOCAL INTEGRAL BASED GRADIENT APPROXIMATION}

Within the FEM, gradient quantities are usually calculated using an approach based on shape function derivatives (see Section 3.6.1). This approach is well understood and established in the literature (Zienkiewicz and Taylor, 1994). Approximating gradient quantities with integrals is a non-standard approach in FEM. Because $\{\operatorname{Curl} \mathbf{H}\} \neq \operatorname{Curl} \mathbf{H}$, it is important to investigate the properties of this integral approximation and compare the approximation against known solutions. As long as the differences between $\{\mathrm{Curl} \mathbf{H}\}$ and Curl $\mathbf{H}$ are not large, then $\{\mathrm{Curl} \mathbf{H}\}$ is a useful tool.

With an understanding about how $\{\mathrm{Curl} \mathbf{H}\}$ behaves, it will be possible to separate physical results predicted with the non-local integral approach from any numerical artifacts.

Within the non-local model, both $\left\{\operatorname{Curl} \mathbf{R}_{h}^{\mathrm{e}}\right\}$ and $\left\{\operatorname{Curl} \mathbf{F}^{\mathbf{P}}\right\}$ are defined. For simplicity, the sensitivity analysis was conducted using a 1D non-local integral based approximation to the gradient. The simplification to $1 \mathrm{D}$ was appropriate because the trends and results in $1 \mathrm{D}$ generalize to $2 \mathrm{D}$ and $3 \mathrm{D}$, and as long as $\{\nabla \mathrm{x}\}$ approximation is reasonable, then the $\{\mathrm{Curl} \mathbf{H}\}$ approximation will also be reasonable. In $1 \mathrm{D}$, the nonlocal integral approximation for a gradient, $\{\nabla \mathrm{x}\}$, becomes

$$
\{\nabla \mathrm{x}\}=\left(\int_{\text {Lcell }}[\mathrm{y}(\mathrm{x}+\xi)-\mathrm{y}(\mathrm{x})] \xi \Theta(\xi) \mathrm{d} \xi\right) \cdot\left(\int_{\text {Lcell }} \xi^{2} \Theta(\xi) \mathrm{d} \xi\right)^{-1}
$$


where $\mathrm{L}_{\text {cell }}$ is the linear integration region. Discretizing space into elements, similar to an FEM mesh, means the integrals in Eq. (5.1) become the summations

$$
\{\nabla x\}=\left(\sum_{n=1}^{\sharp N}[y(x+\xi)-y(x)] \xi \Theta(\xi) \text { Len }\right) \cdot\left(\sum_{n=1}^{\# N} \xi^{2} \Theta(\xi) \text { Len }\right)^{-1}
$$

where Len is the element length. In Eqs. (5.1) and (5.2), there are a large number of possible $\Theta(r)$ functions that could be used. However, only $3 \Theta$ functions were considered in this chapter:

- Exponential. $\Theta=\operatorname{Exp}\left(-\frac{|\xi|}{\omega}\right)$

- Gaussian. $\Theta=\operatorname{Exp}\left(-\frac{\xi^{2}}{\omega^{2}}\right)$

- No damping. $\Theta=1$

The summation based Eq. (5.2) with one of the three $\Theta$ functions was used throughout this chapter in the calculation of $\{\nabla \mathrm{x}\}$.

$\{\nabla \mathrm{x}\}$, defined in Eq. 5.1, approximates the value of Dx at a point. $\{\nabla \mathrm{x}\}$ is defined over the region represented by $\mathrm{d} \xi$ (in Eq. 5.1) or Len (in Eq. 5.2). $\{\nabla \mathrm{x}\}$ and $\nabla \mathrm{x}$ are related: as $\mathrm{d} \xi$ and Len $->0,\{\nabla \mathrm{x}\} \rightarrow \nabla \mathrm{x}$. In this chapter, the accuracy of the nonlocal integral approximation was tested. The effect of different variables (like Len, $\Theta$ function, $\omega$, and mesh refinement) on the gradient approximation $\{\nabla \mathrm{x}\}$ was investigated using both continuous test functions and step test functions. 


\subsection{Evaluation of Continuous Functions}

Since $\mathbf{F}^{\mathbf{P}}$ varies continuously within each grain, testing $\{\nabla \mathbf{x}\}$ against a few continuous functions is important. For the test functions $y=$ constant, $y=m x+b$, and $y=a x^{2}$ $+\mathrm{bx}+\mathrm{c},\{\nabla \mathrm{x}\}=\nabla \mathrm{x}$. For all other functions, $\{\nabla \mathrm{x}\} \neq \nabla \mathrm{x}$.

\subsubsection{The Effect of Integration Volume, Mesh Refinement, and $\Theta$}

$\{\nabla x\}$ of $y=x^{3}$ at $x=0$ was used as an example to illustrate the effects of integration volume (physical dimensions of the neighbor cloud), mesh refinement, and attenuation function $\Theta$ on $\{\nabla \mathrm{x}\}$. To evaluate $\{\nabla \mathrm{x}\}$ at $\mathrm{x}=0$, evenly spaced points between $x=-5$ and $x=5$ were placed on a line segment. These points represented the centriods of the elements used in the gradient calculation. By varying the number of points, the line segment was divided into 11,21, and 41 elements of equal length corresponding to element lengths of $1,0.5$, and 0.25 respectively. The grid with an element length of 1 represented a coarse grid while the grid with an element length of 0.25 represented a fine grid. Figure 5.1 shows the function $y=x^{3}$ plotted over the course grid. 


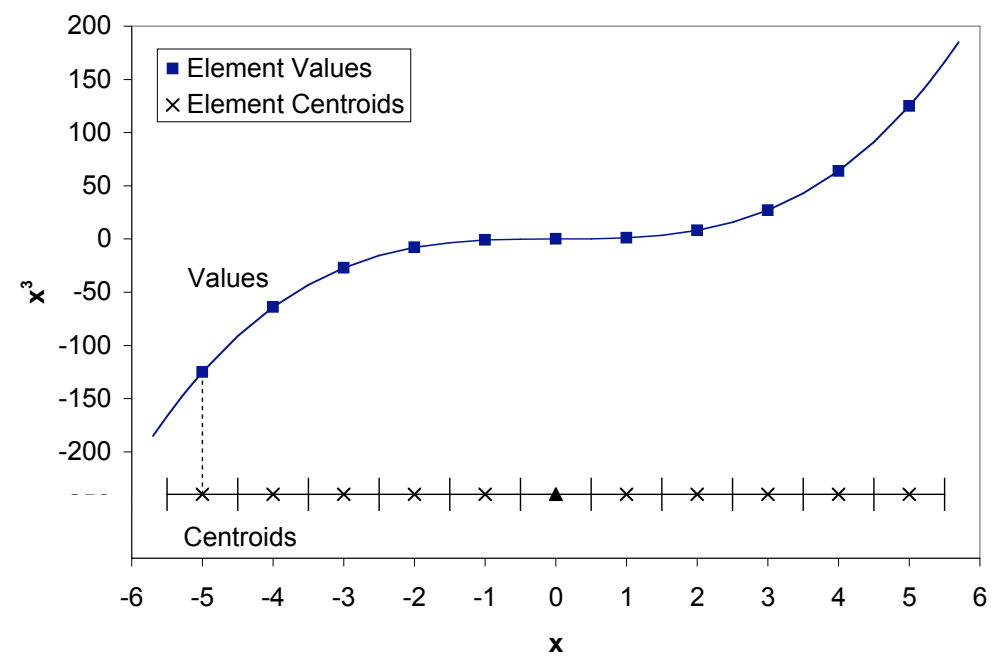

Figure 5.1 - Function $\mathrm{y}=\mathrm{x}^{3}$ plotted over a grid of 11 elements each with a length of 1. $\{\nabla \mathbf{x}\}$ was taken at the element designated by

Equation (5.2) was applied to the grid in Figure 5.1 in the following manner:

- $y(x)=$ the element value for element $\mathbf{\Lambda}$.

- $y(x+\xi)=$ the element value for a neighboring element.

- $\xi=$ the distance between the neighboring element and element $\boldsymbol{\Delta}$. By taking $\{\nabla \mathbf{x}\}$ at $\mathrm{x}=0, \xi=\mathrm{x}$.

The number of neighboring elements included in the $\{\nabla \mathrm{x}\}$ calculation depends on $\mathrm{L}_{\text {cell }}$. An $\mathrm{L}_{\text {cell }}$ of 2 for the grid in Figure 5.1 will include only the 2 nearest neighbors. An $\mathrm{L}_{\text {cell }}$ of 10 will include all 10 of the elements designated with an $\mathrm{x}$. As the mesh is refined, the number of elements captured by $\mathrm{L}_{\text {cell }}$ will increase.

Each of the parameters $L_{\text {cell }}$, mesh refinement (or element size), and $\Theta$ affected $\{\nabla x\}$. The effect of each of these parameter on the error between $\nabla x$ and $\{\nabla x\}$ for $\mathrm{y}=\mathrm{x}^{3}$ at $\mathrm{x}=0$ is shown in Figure 5.2. 


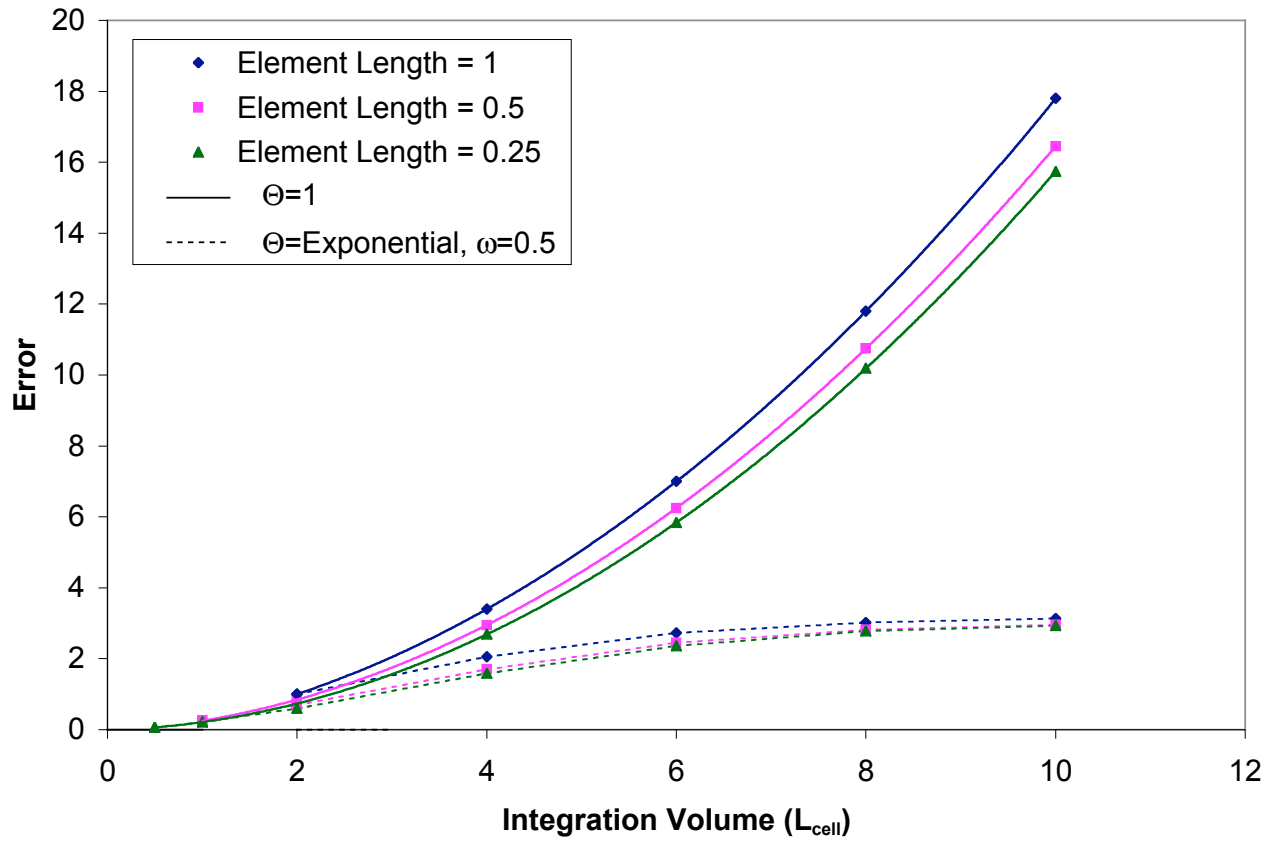

Figure 5.2 - The effects of integration volume, mesh refinement, and $\Theta$ on $\{\nabla x\}$ of $y=x^{3}$. In the exponential attenuation function, $\omega=0.5$.

A number of general trends associated with the non-local integral gradient approximation technique are illustrated in Figure 5.2:

- As the integration volume approaches 0 , the error also approaches 0 .

Conversely, increasing the integration volume increases the error in the gradient approximation.

- Refining the mesh (decreasing the point spacing) decreases the error in the gradient approximation.

- The rate at which the error accumulates depends on the function $\Theta$. For the Exponential and Gaussian $\Theta$ functions, the error accumulation rate depends on $\omega$. The affects of $\omega$ are further explored in Section 5.1.3 
Considering that a derivative is defined on an infinitesimal volume around a point, it is not surprising the error in the gradient approximation goes to zero as the integration volume approaches an infinitesimal volume around the point of interest.

The error in $\{\nabla \mathrm{x}\}$ for $\mathrm{y}=\mathrm{x}^{3}$ becomes substantial as the integration volume increases. This error is not a concern for the non-local model since $\mathbf{F}^{\mathbf{P}}$ does not usually have such steep spatial variations within a grain. A more realistic example of $\mathbf{F}^{\mathbf{P}}$ within a grain would be the test function $\mathrm{y}=\mathrm{x}^{0.5} .\{\nabla \mathrm{x}\}$ was evaluated at $\mathrm{x}=5$ and at $\mathrm{x}=15$ on a grid with elements of length $1,0.5$, and 0.25 . Figures 5.3 and 5.4 show the function $y=x^{0.5}$ centered at $\mathrm{x}=5$ and $\mathrm{x}=15$ respectively plotted over a line segment with elements of length 0.25 . The effects of integration volume, mesh refinement, and $\Theta$ with on $\{\nabla x\}$ calculated at $x=5$ and $x=15$ are shown in Figure 5.5 and Figure 5.6 respectively. 


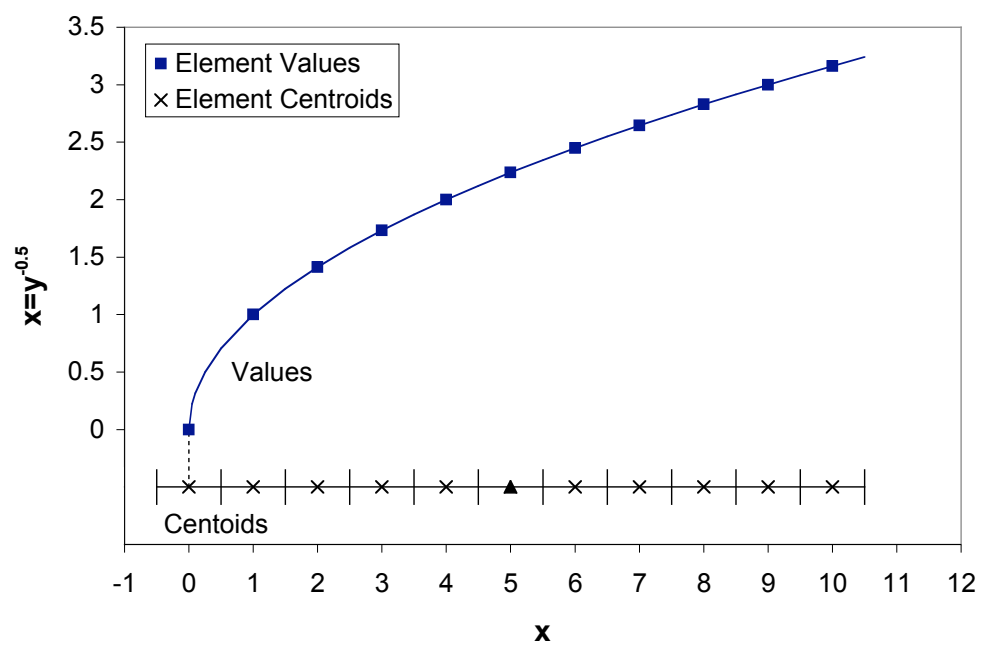

Figure 5.3 - Function $y=x^{-0.5}$ between $x=0$ and $x=10$ plotted over a grid of 11 elements each with a length of $1 .\{\nabla \mathbf{x}\}$ was taken at the element designated by $\mathbf{\Delta}$.

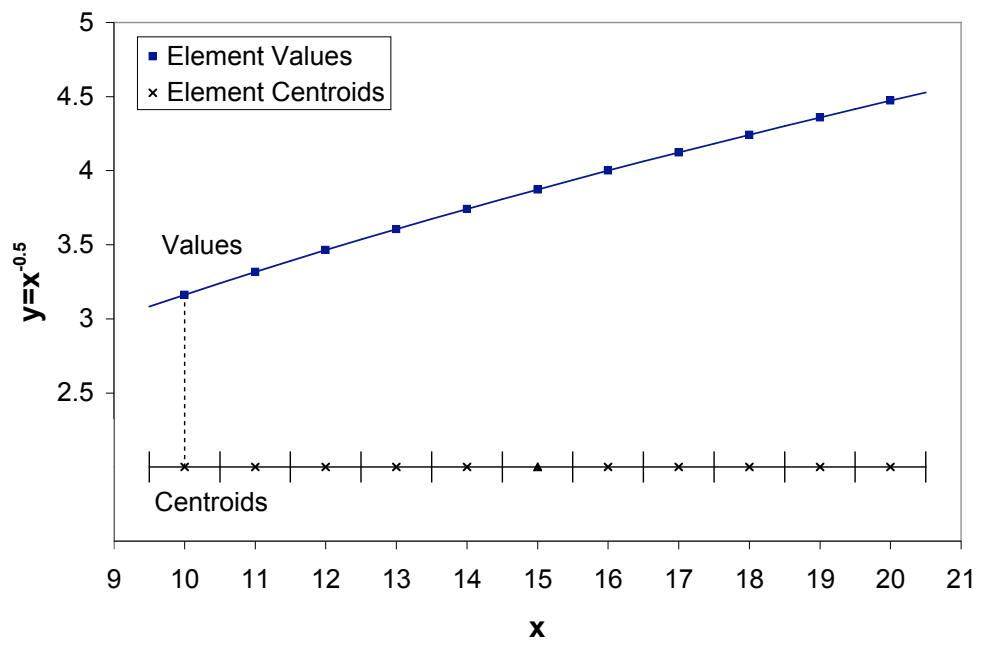

Figure $5.4-$ Function $y=x^{-0.5}$ between $x=10$ and $x=20$ plotted over a grid of 41 elements each with a length of $1 .\{\nabla \mathbf{x}\}$ was taken at the element designated by $\boldsymbol{\Lambda}$. 


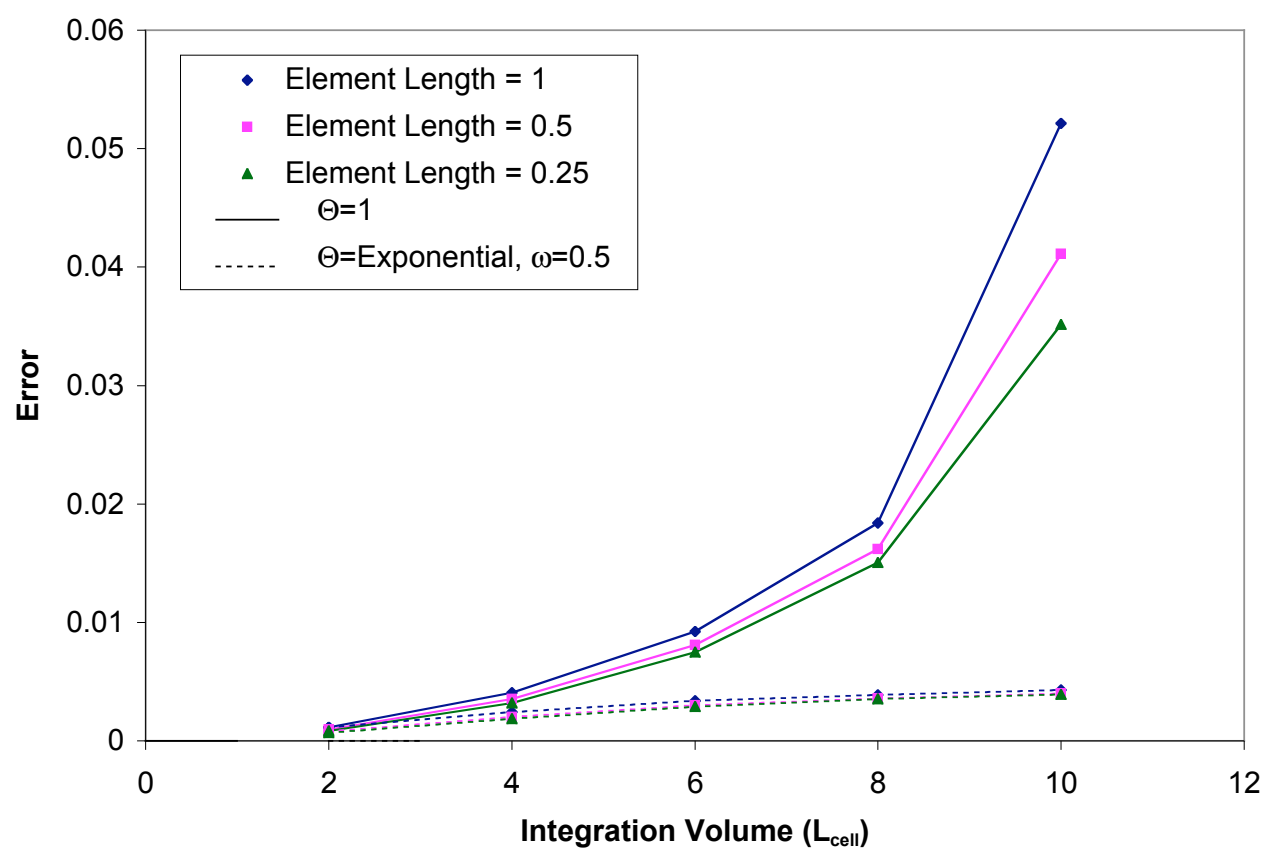

Figure 5.5 - The effects of integration volume, mesh refinement, and $\Theta$ on $\{\nabla \mathrm{x}\}$ at $\mathrm{x}=5$ for $\mathrm{y}=\mathrm{x}^{-0.5}$. In the exponential attenuation function, $\omega=0.5$.

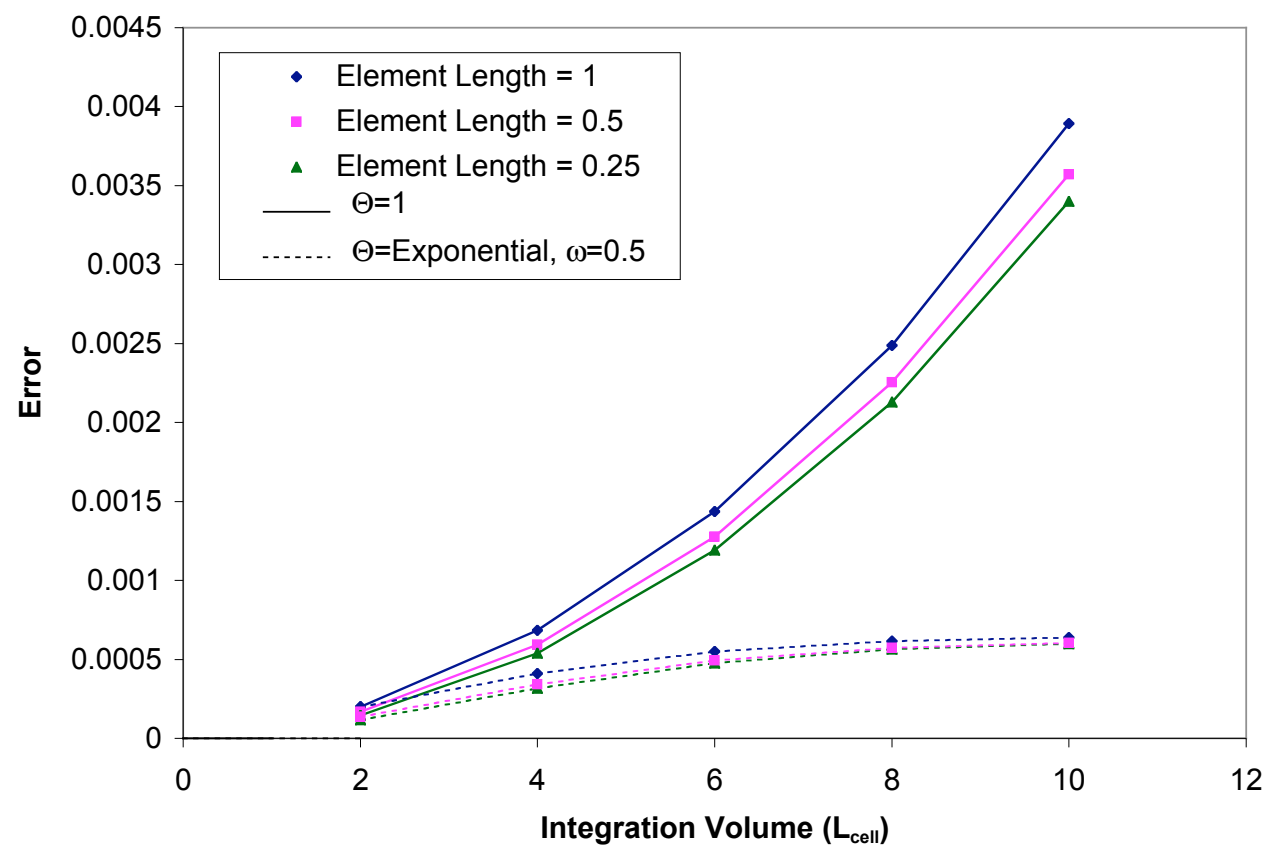

Figure 5.6 - The effects of integration volume, mesh refinement, and $\Theta$ on $\{\nabla x\}$ at $x=10$ for $\mathrm{y}=\mathrm{x}^{-0.5}$. In the exponential attenuation function, $\omega=0.5$. 
The error in $\{\nabla \mathrm{x}\}$ for $\mathrm{y}=\mathrm{x}^{0.5}$ is substantially less than the error for $\mathrm{y}=\mathrm{x}^{3}$. With an integration volume of 10 and $\Theta=1$, the error in $\{\nabla \mathrm{x}\}$ for $\mathrm{y}=\mathrm{x}^{3}$ at $\mathrm{x}=0$ was 17.8 while the error in $\{\nabla \mathrm{x}\}$ for $\mathrm{y}=\mathrm{x}^{0.5}$ at $\mathrm{x}=5$ was 0.0523 and at $\mathrm{x}=15$ was 0.0039 . The error in $\{\nabla \mathrm{x}\}$ is lower with $\Theta=\operatorname{Exp}\left(-\frac{|\xi|}{\omega}\right)$. For $\mathrm{y}=\mathrm{x}^{3}$ at $\mathrm{x}=0$, the error is 3.13 , while for $\mathrm{y}=\mathrm{x}^{0.5}$ at $\mathrm{x}=5$ the error is 0.00431 and at $x=15$ the error is 0.00064 . Notice that the error is at least 3 orders of magnitudes less for the function $y=x^{-0.5}$ compared to $y=x^{3}$. The error is another order of magnitude less for the function $\mathrm{y}=\mathrm{x}^{-0.5}$ at $\mathrm{x}=5$ compared to $\mathrm{x}=15$.

The major difference between these 3 cases illustrated in this section is the amount of curvature contained within the integration volume. The curvature is greatest for the function $\mathrm{y}=\mathrm{x}^{3}$ between -5 and 5 and least for $\mathrm{y}=\mathrm{x}^{-0.5}$ between 10 and 20. These results lead to the conclusion that the error in $\{\nabla \mathrm{x}\}$ depends on curvature: lower curvature leads to a lower error in $\{\nabla \mathrm{x}\}$. This is an important point. The non-local integral approximation has its limitations, but the spatial variations of $\mathbf{F}^{\mathbf{P}}$ inside the grain are relatively gentle. Thus the non-local integral approximation is an appropriate tool for the problems in this research project.

\subsubsection{The Effect of a Non-Uniform Mesh}

The examples above all utilized a mesh composed of uniformly sized elements. In FEM, meshes are generally not uniform but rather composed of elements of varying sizes. Thus it is important to investigate if and how a non-uniform mesh will affect $\{\nabla \mathrm{x}\}$. To this end, the gradient at $\mathrm{x}=0$ for test function $\mathrm{y}=\mathrm{x}^{3}$ was again evaluated. The 
initial grid was a line segment with 5 element centroids evenly spaced between $x=-2$ and $x=2$. The non-uniform mesh was created from the evenly spaced mesh by subdividing the two elements at -1 and -2 into $3,6,12,15$, and 30 elements. As more subdivisions are introduced, the average element size decreases. In Figures 5.7 and 5.8, the function $\mathrm{y}=\mathrm{x}^{3}$ is plotted over the grid divided into 5 evenly spaced elements, and the grid with the two elements at $x=-1$ and $x=-2$ subdivided into 12 elements.

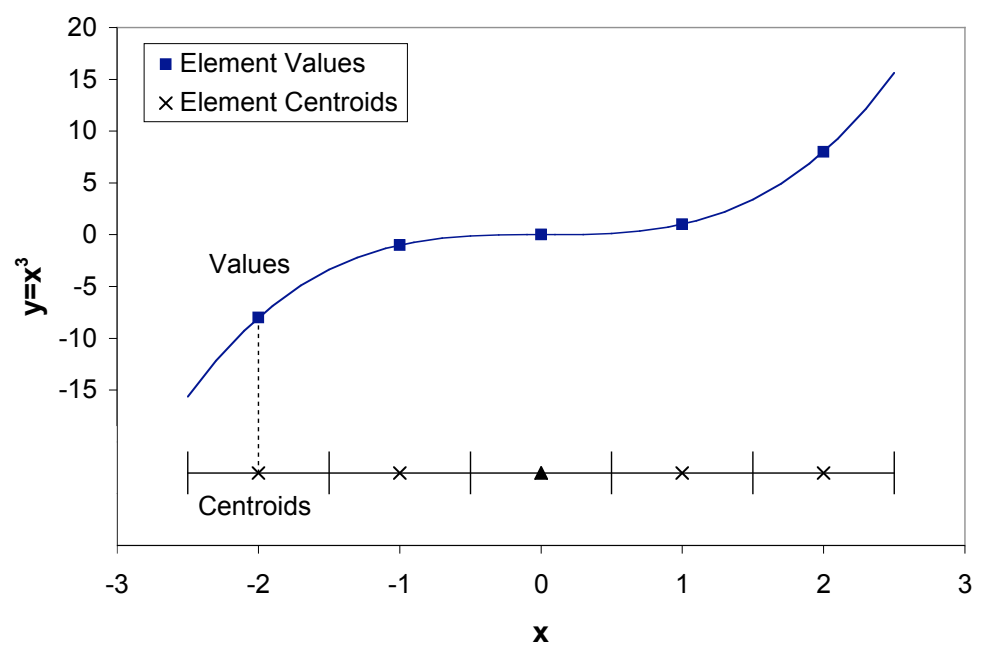

Figure 5.7 - Function $\mathrm{y}=\mathrm{x}^{-0.5}$ between $\mathrm{x}=-2$ and $\mathrm{x}=2$ plotted over a grid with an average element size of $1 .\{\nabla \mathrm{x}\}$ was taken at the element designated by 


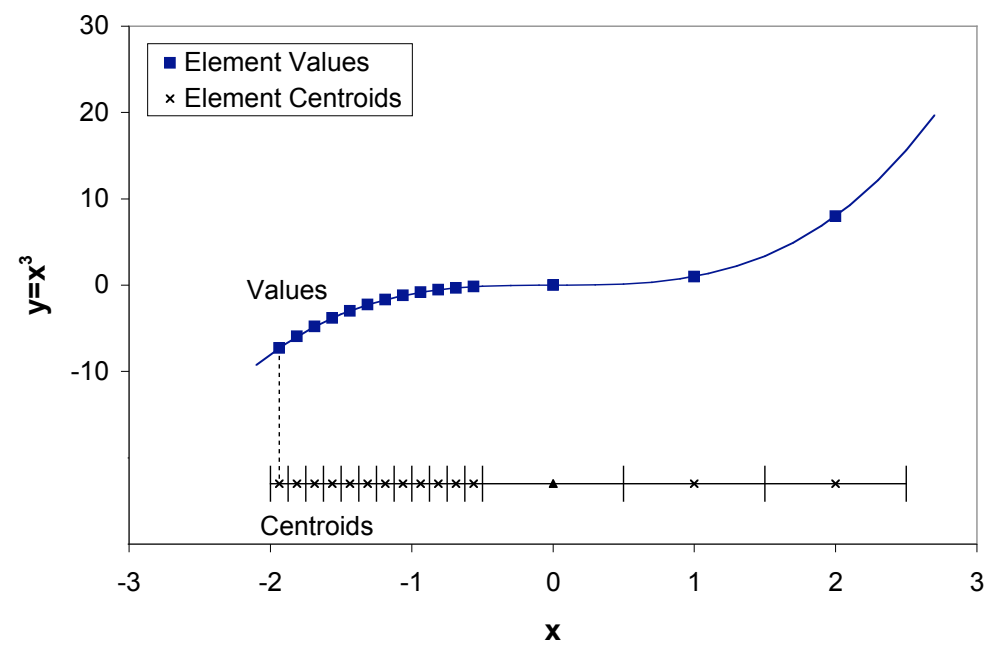

Figure 5.8 - Function $\mathrm{y}=\mathrm{x}^{-0.5}$ between $\mathrm{x}=-2$ and $\mathrm{x}=2$ plotted over a grid with an average element size of 0.3. $\{\nabla \mathrm{x}\}$ was taken at the element designated by $\mathbf{\Lambda}$.

The effects of decreasing element size on the error in $\{\nabla \mathrm{x}\}$ are plotted in Figure 5.9.

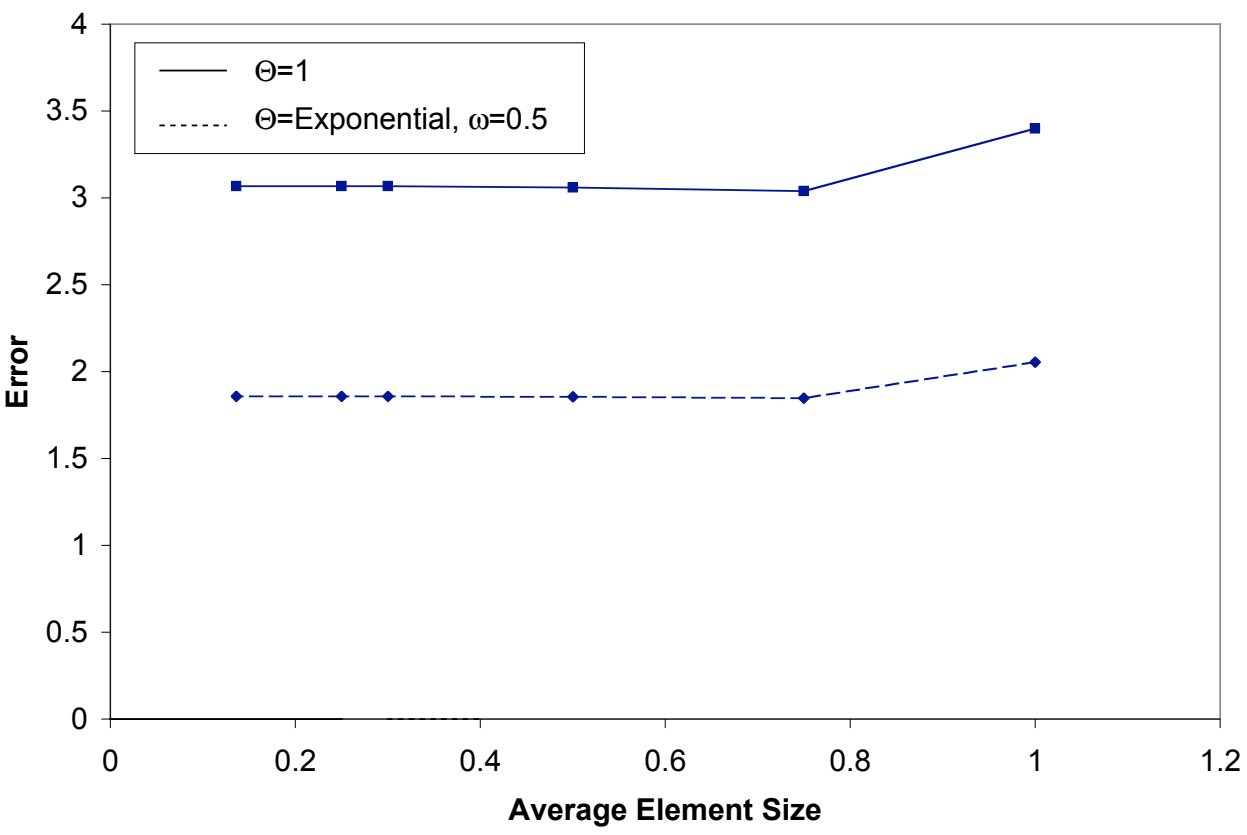

Figure 5.9 - The effect of non-symmetric mesh on $\{\nabla x\}$ for $y=x^{3}$ at $x=0$. In the exponential attenuation function, $\omega=0.5$. 
The important point in Figure 5.9 is that non-uniformity in the mesh does not introduce additional error into $\{\nabla \mathbf{x}\}$. For this simple example, refining part of the mesh decreased the error in $\{\nabla \mathrm{x}\}$, although the decrease in the error quickly saturated as the average element size got smaller. These results suggest that a fine mesh in the regions of high curvature combined with a coarser mesh in regions with less curvature may yield the best comprise between computational expense and accuracy for $\{\nabla \mathbf{x}\}$.

\subsubsection{The Effect of $\omega$}

Another important parameter in the non-local integral based gradient approximation is $\omega$. Changing $\omega$ changes how much points contribute to the gradient approximation: as $\omega$ decreases, points closer to $\mathrm{x}$ contribute more to the gradient than points far away. This point is illustrated by Figures 5.10 and 5.11, which show plots of $\Theta=\operatorname{Exp}\left(-\frac{|\xi|}{\omega}\right)$ and $\Theta=\operatorname{Exp}\left(-\frac{\xi^{2}}{\omega^{2}}\right)$ with various values of $\omega$. 


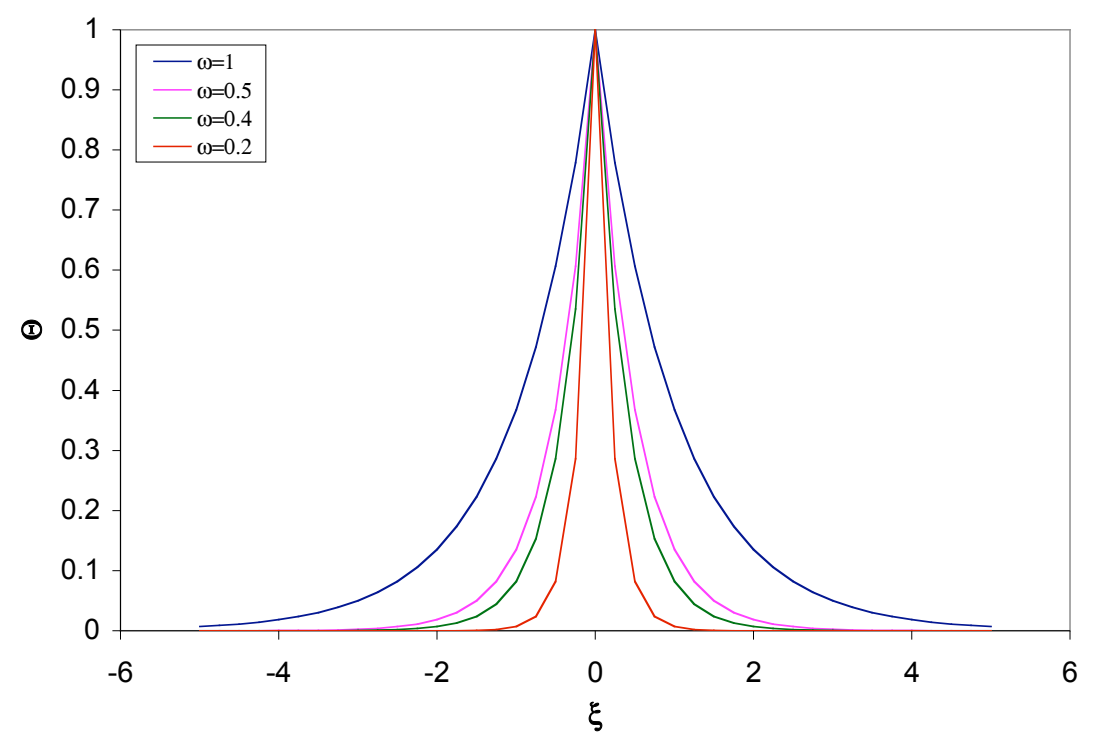

Figure $5.10-$ The attenuation function, $\Theta=\operatorname{Exp}\left(-\frac{|\xi|}{\omega}\right)$, plotted between $\xi=-5$ and $\xi=5$.

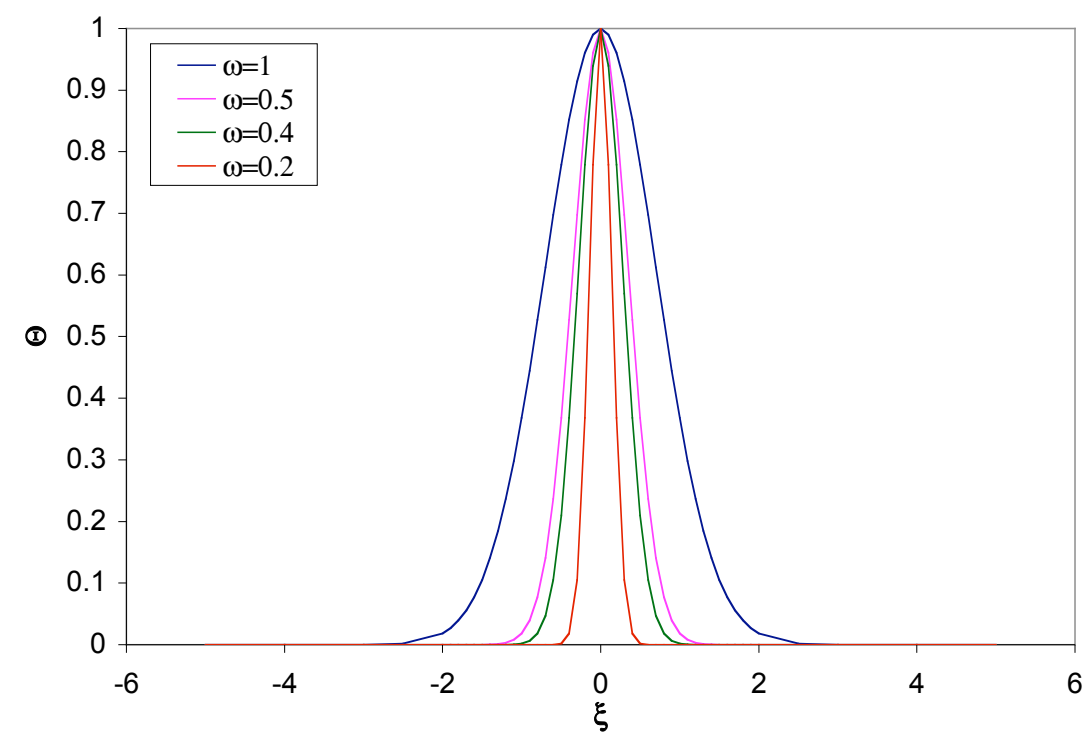

Figure $5.11-$ The attenuation function, $\Theta=\operatorname{Exp}\left(-\frac{\xi^{2}}{\omega^{2}}\right)$, plotted between $\xi=-5$ and $\xi=5$. 
For each value of $\omega$ in Figure 5.10, $\Theta$ gets smaller as $\zeta$ moves away from 0 . Also, as $\omega$ increases $\Theta$ approaches 0 faster. These trends also apply to $\Theta=\operatorname{Exp}\left(-\frac{\xi^{2}}{\omega^{2}}\right)$. The difference between the Exponential and Gaussian $\Theta$ is the shape of the $\Theta$ vs. $\xi$ curve. Thus, the $\omega$ in both the Gaussian and Exponential $\Theta$ functions controls the range over which $\{\nabla \mathrm{x}\}$ is defined.

The results presented in Figures 5.2, 5.5, 5.6, and 5.9 all utilized $\omega=1$ for convenience. The effects of $\omega$ on $\{\nabla x\}$ were investigated with the test function $y=x^{3}$ on the coarse mesh in Figure 5.1. Figures 5.12 and 5.13 show the effect of $\omega$ in $\Theta=\operatorname{Exp}\left(-\frac{|\xi|}{\omega}\right)$ on $\{\nabla \mathrm{x}\}$ 


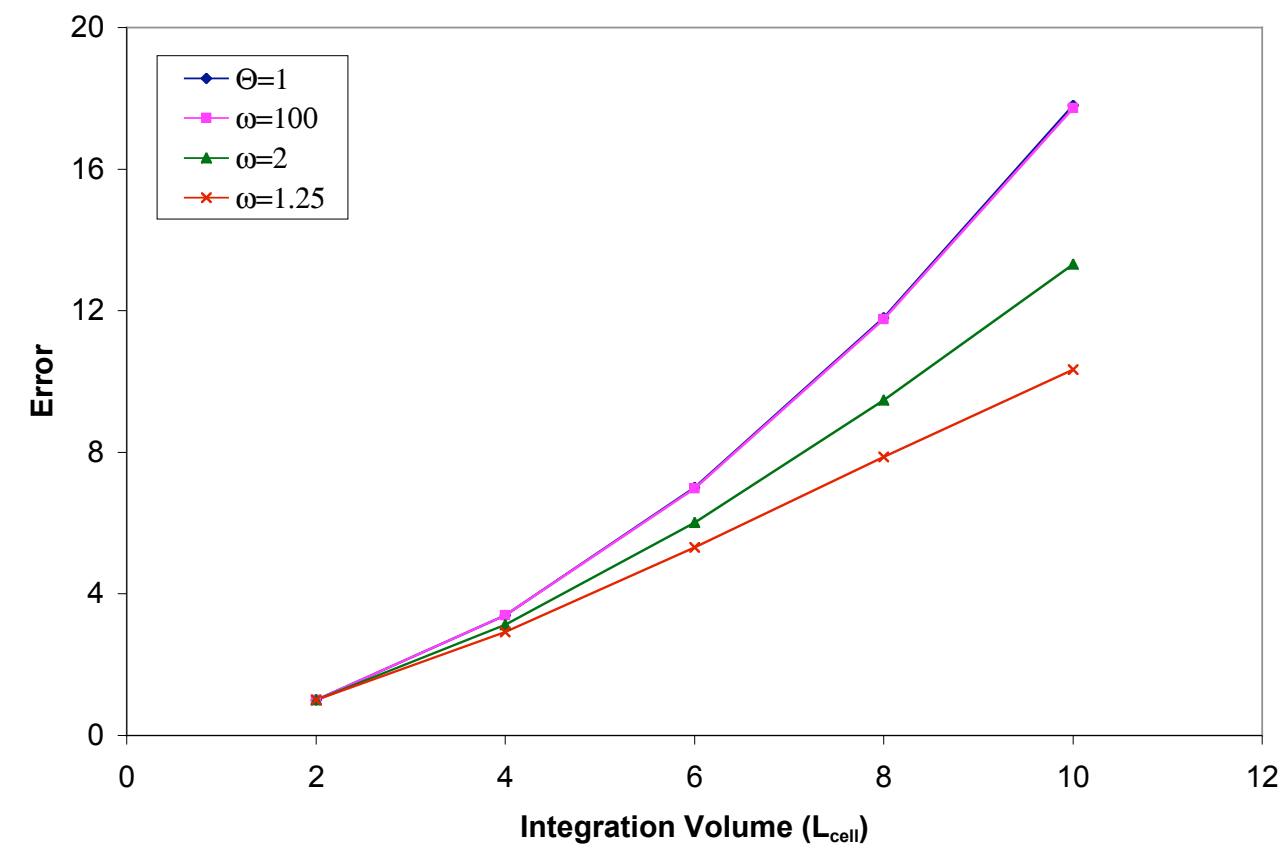

Figure 5.12 - The effect of the $\omega$ in $\Theta=\operatorname{Exp}\left(-\frac{|\xi|}{\omega}\right)$ on $\{\nabla x\}$ for $y=x^{3}$ at $x=0$.

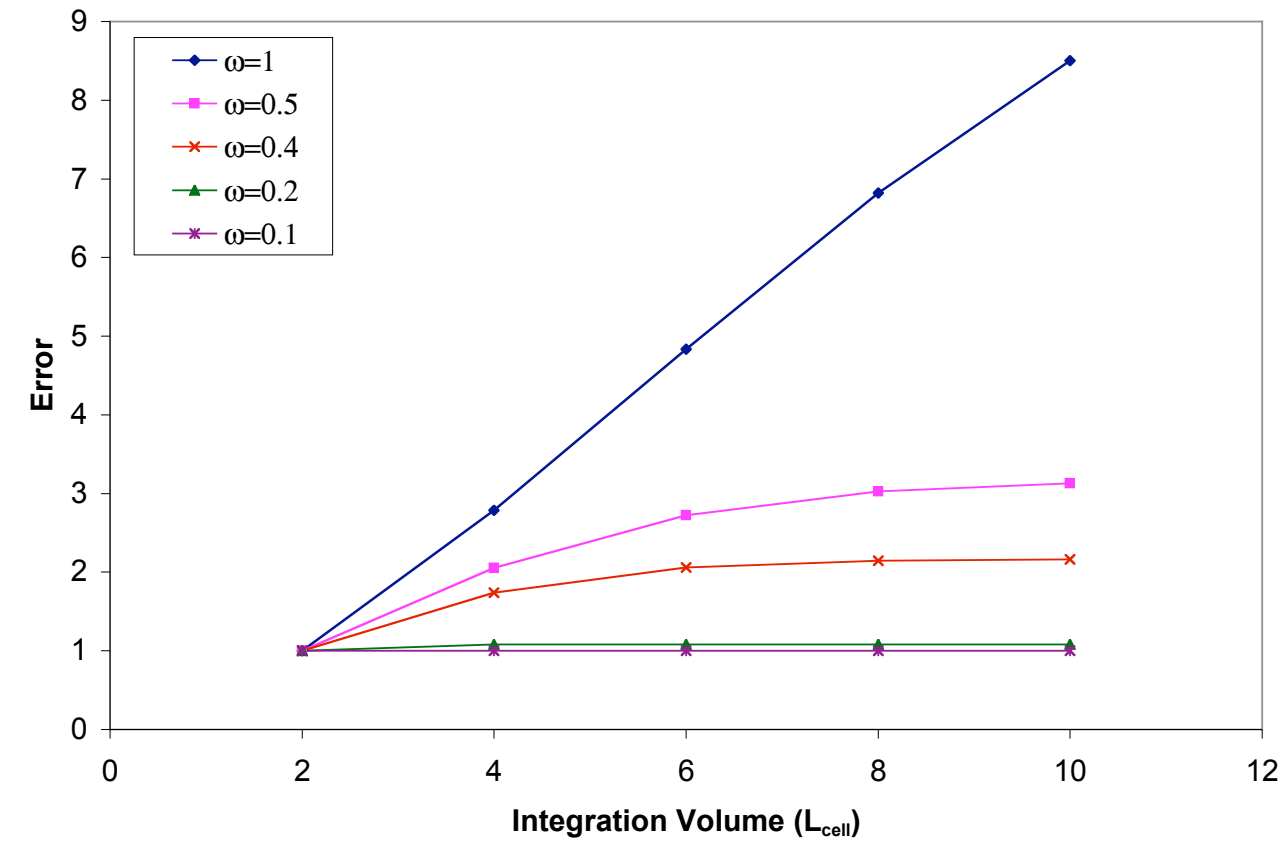

Figure 5.13 - The effect of the $\omega$ in $\Theta=\operatorname{Exp}\left(-\frac{|\xi|}{\omega}\right)$ on $\{\nabla x\}$ for $y=x^{3}$ at $x=0$. 
Figures 5.12 and Fig 5.13 show that in general increasing $\omega$ decreases the error in the $\{\nabla \mathbf{x}\}$ calculation. These figures also illustrate the limits of $\omega$ and $\Theta$ on the $\{\nabla x\}$

calculation. In Figure 5.12, $\omega=0.01$ is too small for integration volumes between 2 and 10 resulting in almost no damping on any element. In this under-damped case, the error for $\{\nabla x\}$ with $\omega=0.01$ and $\{\nabla x\}$ without $\Theta$ are nearly the same. In Figure 5.13, $\omega=5$ is too large resulting in a large amount of damping even in the closest elements. In this over-damped case, the error in $\{\nabla x\}$ is almost constant. Notice that as $\omega \rightarrow \infty$, the error in $\{\nabla \mathrm{x}\}$ does not go to zero. This phenomenon is explained in Section 5.3.

The optimal $\omega$ will show an initial increase in the error that levels off as the integration volume gets larger. For $\mathrm{L}_{\text {cell }}$ containing 10 elements, the optimal $\omega$ is between 2.0 and 2.5. In Figure 5.13, the error for $\omega=2.0$ and $\omega=2.5$ initially increases indicating that elements close to $\mathrm{x}=0$ contribute to $\{\nabla \mathrm{x}\}$. As the integration volume increases, the error levels off because $\Theta$ is damping out the contributions from elements at the outer edges of the neighbor cloud.

\subsection{Evaluation of Step Functions}

While the $\mathbf{F}^{\mathbf{P}}$ and $\mathbf{R}_{\mathbf{h}}^{\mathbf{e}}$ fields are continuous throughout the grain, these fields resemble a discontinuous step function at the grain boundary. The behavior of the nonlocal integral approximation in the vicinity of a step function is important because this behavior will ultimately affect $\rho^{\mathrm{GND}}$ and $\tau^{\mathrm{CSS}}$ within the material body. The true gradient, 
$\nabla \mathrm{x}$, of a step function is the $\delta$ function; at the discontinuity the gradient is infinity. $\{\nabla \mathrm{x}\}$ of a step function does not return the $\delta$ function because $\{\nabla \mathrm{x}\}$ is not evaluated at the discontinuity, but rather a small distance away from it.

In order to investigate the behavior of $\{\nabla \mathrm{x}\}$, a $1 \mathrm{D}$ case was considered using the step function

$$
y=\left\{\begin{array}{ll}
4 & x<7.5 \\
9 & x>7.5
\end{array}\right\}
$$

In this example a line segment running from $\mathrm{x}=1$ to $\mathrm{x}=14$ was subdivided into 14 elements each with a length of 1 as shown in Figure 5.14.

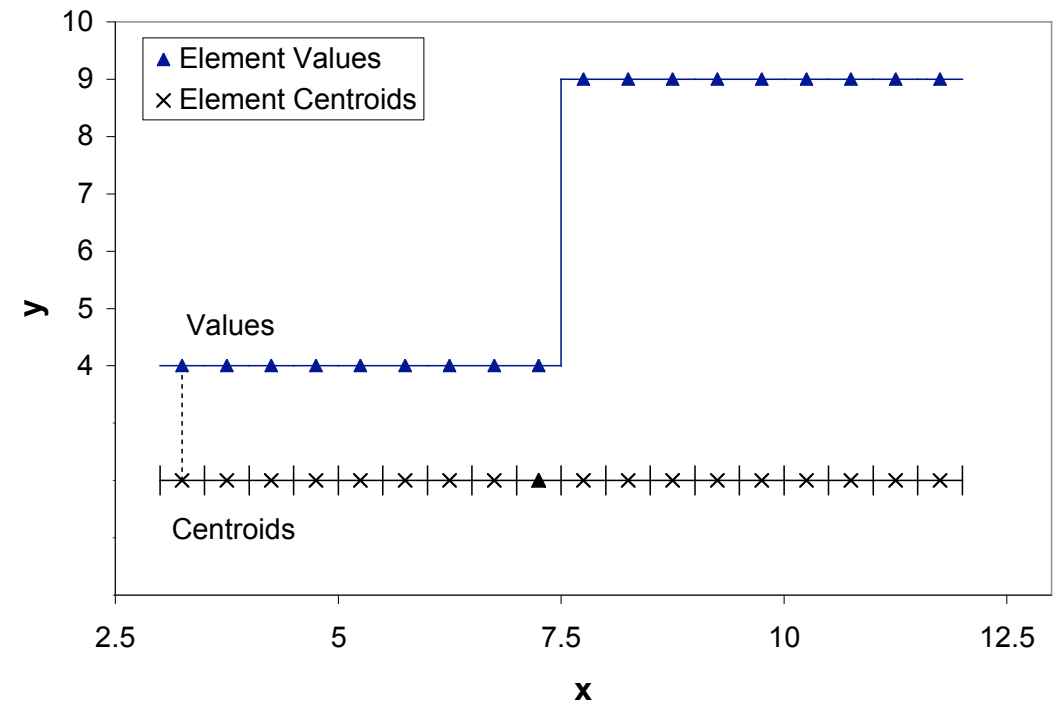

Figure 5.14 - The step-function $y=\left\{\begin{array}{ll}4 & x<7.5 \\ 9 & x>7.5\end{array}\right\}$ between $x=1$ and $x=14$ plotted over a grid with an average element size of $1 .\{\nabla x\}$ was taken at the element designated by $\mathbf{\Lambda}$. 


\subsubsection{The Effect of $\Theta$ Function Type}

$\{\nabla x\}$ was calculated using the function and grid shown in Figure 5.14. At each element between 3.25 and $11.75,\{\nabla x\}$ was determined using each of the $3 \Theta$ 's (constant, Gaussian, and Exponential) with $\omega=1$ and an integration volume of 8 . The resulting $\{\nabla \mathbf{x}\}$ is plotted at each element centroid in Figure 5.15.

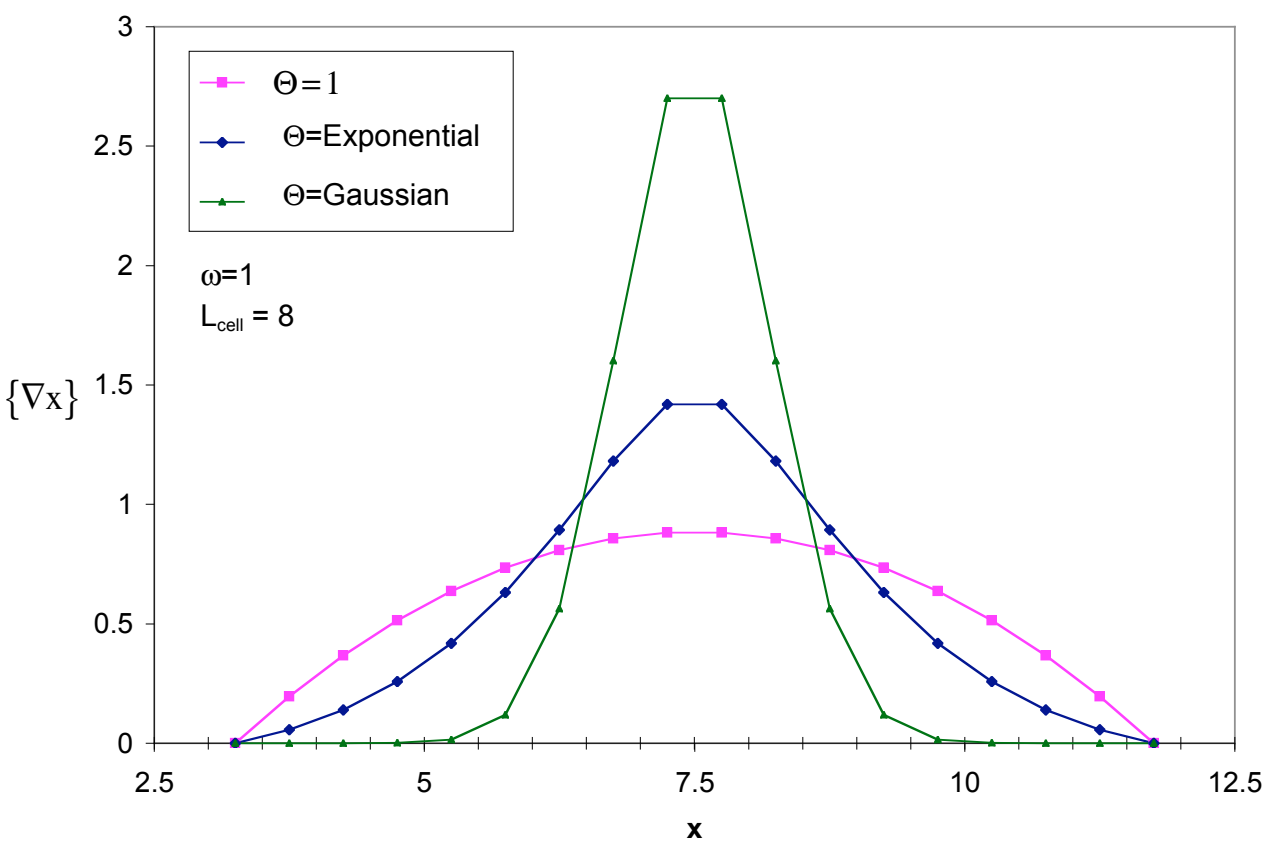

Figure 5.15 - The effect of $\Theta$ on $\{\nabla x\}$ of a step-function

The effect of $\Theta$ on the $\{\nabla x\}$ of a step function is different than the effect of $\Theta$ on the $\{\nabla \mathrm{x}\}$ of continuous functions. $\Theta$ limits the error in $\{\nabla \mathrm{x}\}$ when applied to continuous functions. $\Theta$ defines the shape of the $\{\nabla \mathrm{x}\}$ profile when applied to step functions. For 
each $\Theta$ in Figure 5.15, $\{\nabla x\}=0$ when $x \leq 3.25$ and $x \geq 11.75$ because the neighbor clouds for these points do not include any points on the other side of the discontinuity. However, $\Theta$ controls the rate at which the gradient approaches 0 . For $\Theta=1$, the gradient profile is circular in shape, but the exponential and Gaussian $\Theta$ have a very different shape that resembles $1 / \mathrm{r}$.

The shape of the gradient profile defined by $\Theta$ is very important because the $\{\nabla \mathrm{x}\}$ profile is directly related to the $\rho^{\mathrm{GND}}$ and $\tau^{\mathrm{CSS}}$ profiles. The direct relation between the $\{\nabla \mathrm{x}\}$ and the $\rho^{\mathrm{GND}}, \tau^{\mathrm{CSS}}$ profiles makes the $\Theta$ functions more than just mathematical damping functions. These $\Theta$ functions have physical meaning in the non-local model; they define how orientation discontinuity at the grain boundary affects $\rho^{\mathrm{GND}}$ and $\tau^{\mathrm{CSS}}$ in the vicinity of the grain boundary.

This ability to define the $\rho^{\mathrm{GND}}$ and $\tau^{\mathrm{CSS}}$ profiles in the vicinity of grain boundaries is the primary advantage of the non-local integral based gradient approximation over the shape function derivative approach. In the shape function derivative approach, a gradient exits only in those elements with nodes at the discontinuity. In the example illustrated in Figure 5.14, the gradient would be defined in the element designated by $\boldsymbol{\Delta}$ and the element to its right as shown in Figure 5.16. 


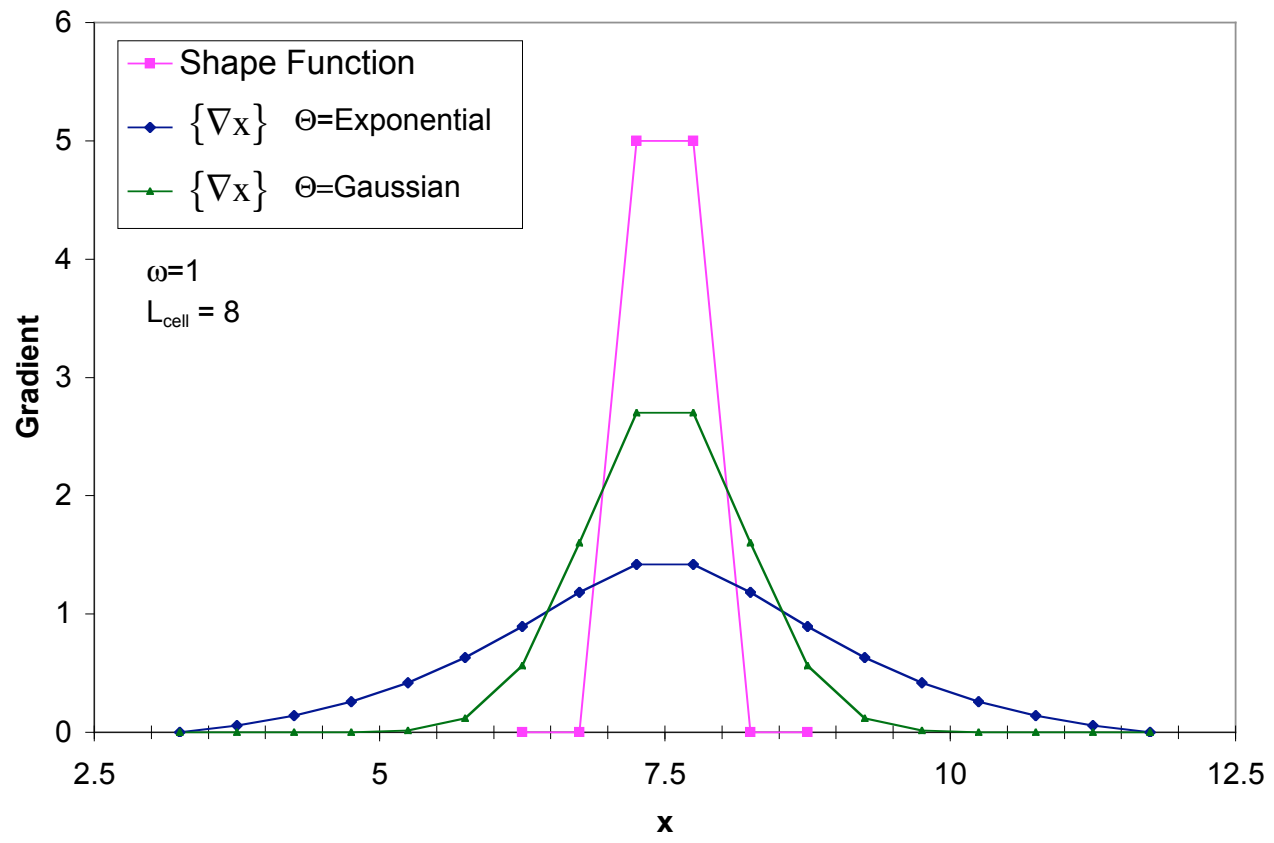

Figure 5-16 - The gradient calculated with the non-local integral based approach and the shape function based approach.

Rather than the gradual decay in $\rho^{\mathrm{GND}}$ and $\tau^{\mathrm{CSS}}$ predicted with the non-local integral approach, the shape function approach calculates a maximum value in the two elements adjacent to the discontinuity that decays to zero over one element.

\subsubsection{The Effect of Integration Volume}

The effect of increasing the integration volume on $\Theta=1$ and $\Theta=\operatorname{Exp}\left(-\frac{|\xi|}{\omega}\right)$ was tested using Eq. 5.3 and the grid shown in Figure 5.14. $\{\nabla \mathrm{x}\}$ was calculated with $\omega=1$ and integration volumes of $1,2,4$, and 8 . The resulting $\{\nabla x\}$ is plotted at each element 
centroid for $\Theta=1$ in Figure 5.17 and $\Theta=\operatorname{Exp}\left(-\frac{|\xi|}{\omega}\right)$ in Figure 5.18. Increasing the integration volume when calculating $\{\nabla \mathrm{x}\}$ of a step function has two primary effects:

- Increasing the gradient affected zone. The gradient affected zone is the region around the step function that has a non-zero $\{\nabla \mathrm{x}\}$. The larger the integration volume, the farther away from the step function discontinuity a point can be and still include points on the other side of the discontinuity.

- Decreasing the maximum value of the gradient. Each new element added to the neighbor cloud contributes to the denominator. But only elements on one side of the discontinuity contribute to the numerator.

Despite the changing shape, the area under each curve in Figure 5.17 and 5.18 is the same. Also note that the $\{\nabla \mathrm{x}\}$ for $\mathrm{L}_{\text {cell }}=1.0$ is exactly the same as the gradient profile calculated using shape function derivatives.

Here again it is important to interpret these results in terms of $\rho^{\mathrm{GND}}$ and $\tau^{\mathrm{CSS}}$. A larger integration volume will result in a larger grain boundary hardened region. However, the peak value of $\rho^{\mathrm{GND}}$ and $\tau^{\mathrm{CSS}}$ with the hardened grain boundary region decreases as the integration volume decreases. 


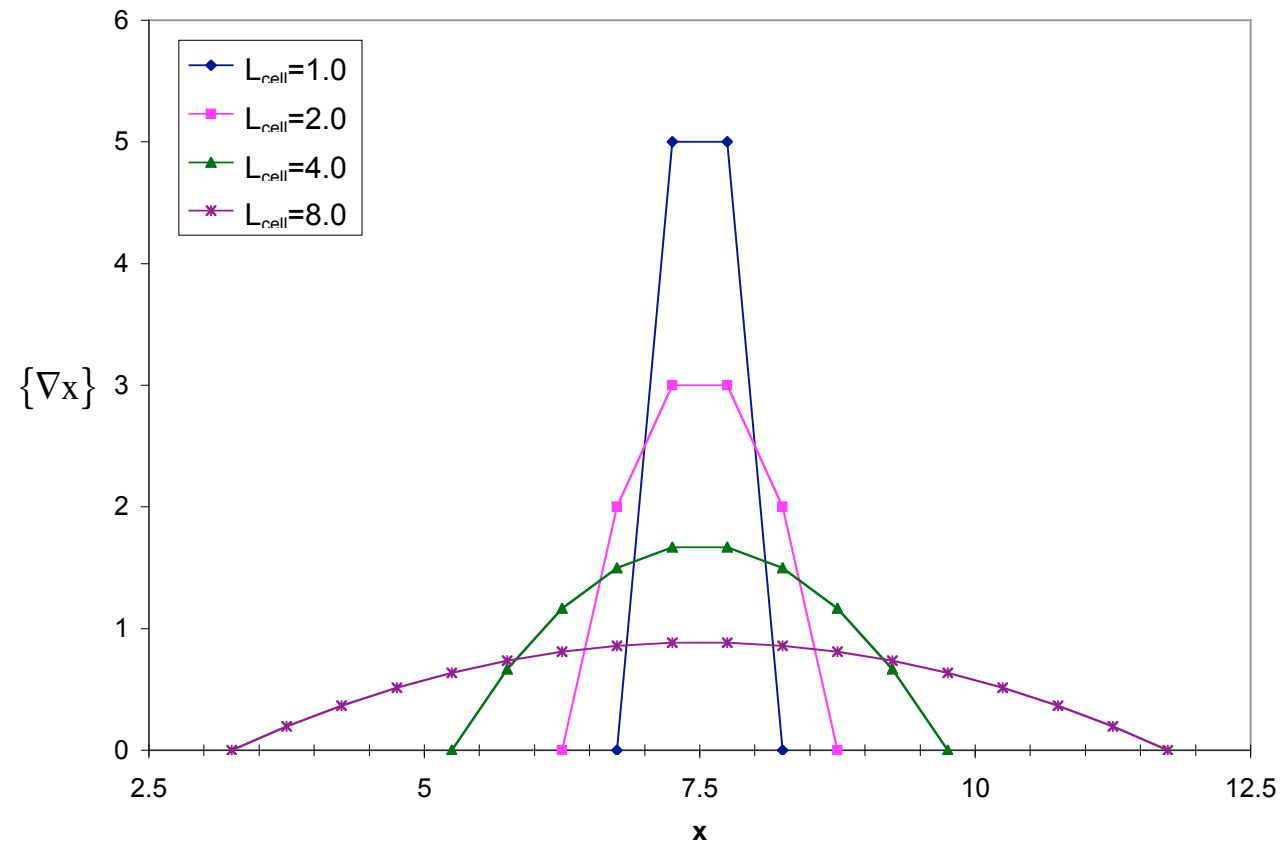

Figure 5.17 - The effect of integration volume on $\{\nabla \mathrm{x}\}$ of a step function using $\Theta=1$.

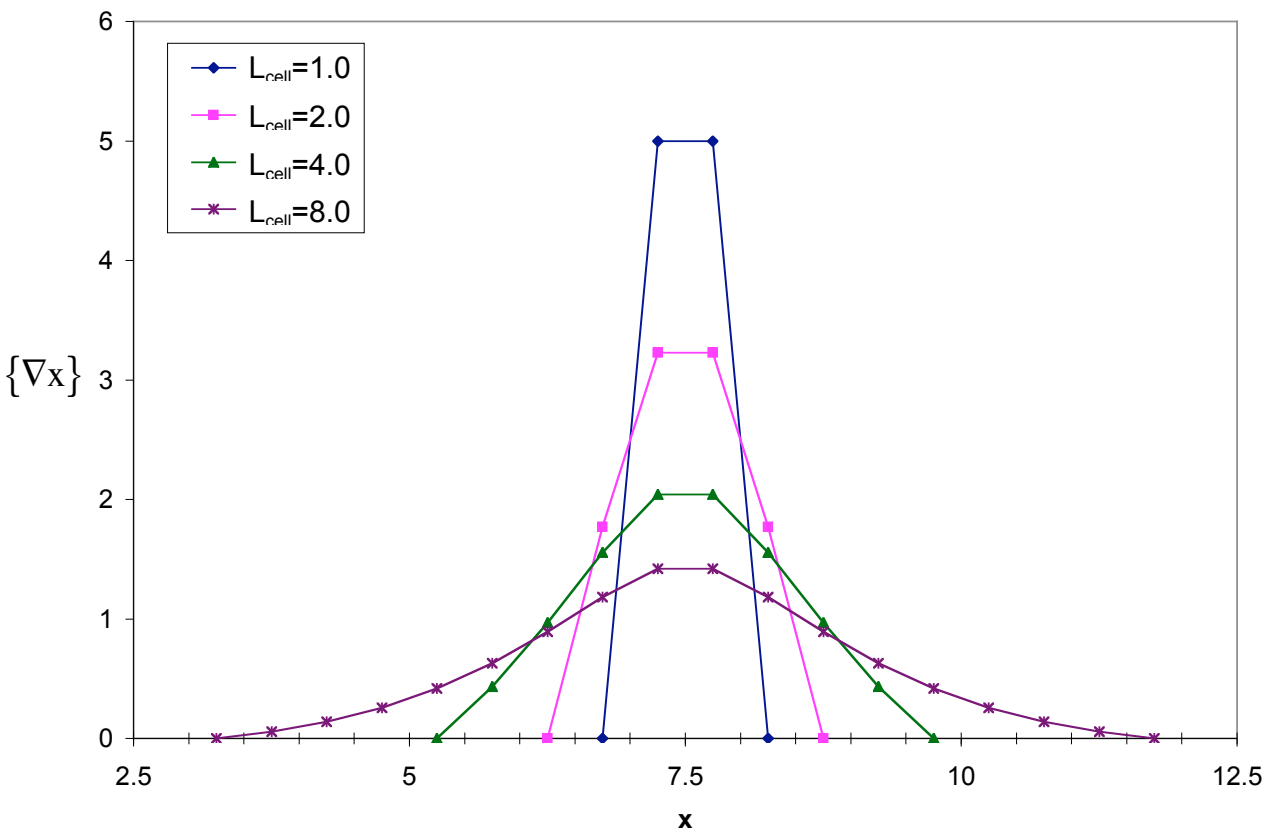

Figure 5.18 - The effect of integration volume on $\{\nabla \mathrm{x}\}$ of a step function

$$
\text { using } \Theta=\operatorname{Exp}\left(-\frac{|\xi|}{\omega}\right) \text {. }
$$


When the integration volume is small $\left(\mathrm{L}_{\text {cell }}=1.0\right.$ or 2.0$)$, the gradient profiles for $\Theta=1$ and $\Theta=\operatorname{Exp}\left(-\frac{|\xi|}{\omega}\right)$ are not very different. The reason for the similarities at $L_{\text {cell }}=$ 1 is that this integration volume contains only the nearest 2 neighbors. $\{\nabla \mathrm{x}\}$ for nearest neighbor integration volumes converges to the same value regardless of $\Theta$. See Section 5.3 for details on this phenomenon. The similarities for $\mathrm{L}_{\text {cell }}=2.0$ is due to $\Theta$. When the value of $\Theta$ throughout both integration volumes is similar, both $\{\nabla \mathrm{x}\}$ profiles will be similar. In this case, $\Theta=\operatorname{Exp}\left(-\frac{|\xi|}{\omega}\right)$ is almost 1 throughout the integration volume. In general, an integration volume is considered too small if one or both of these effects are present.

\subsubsection{The Effect of $\omega$}

The affect of $\omega$ on $\{\nabla x\}$ is very similar to the effect of integration volume shown in the previous section. Using the same grid (Figure 5.16) and constant integration volume of $8,\{\nabla x\}$ was calculated for various values of $\omega$ as shown in Figure 5.19. 


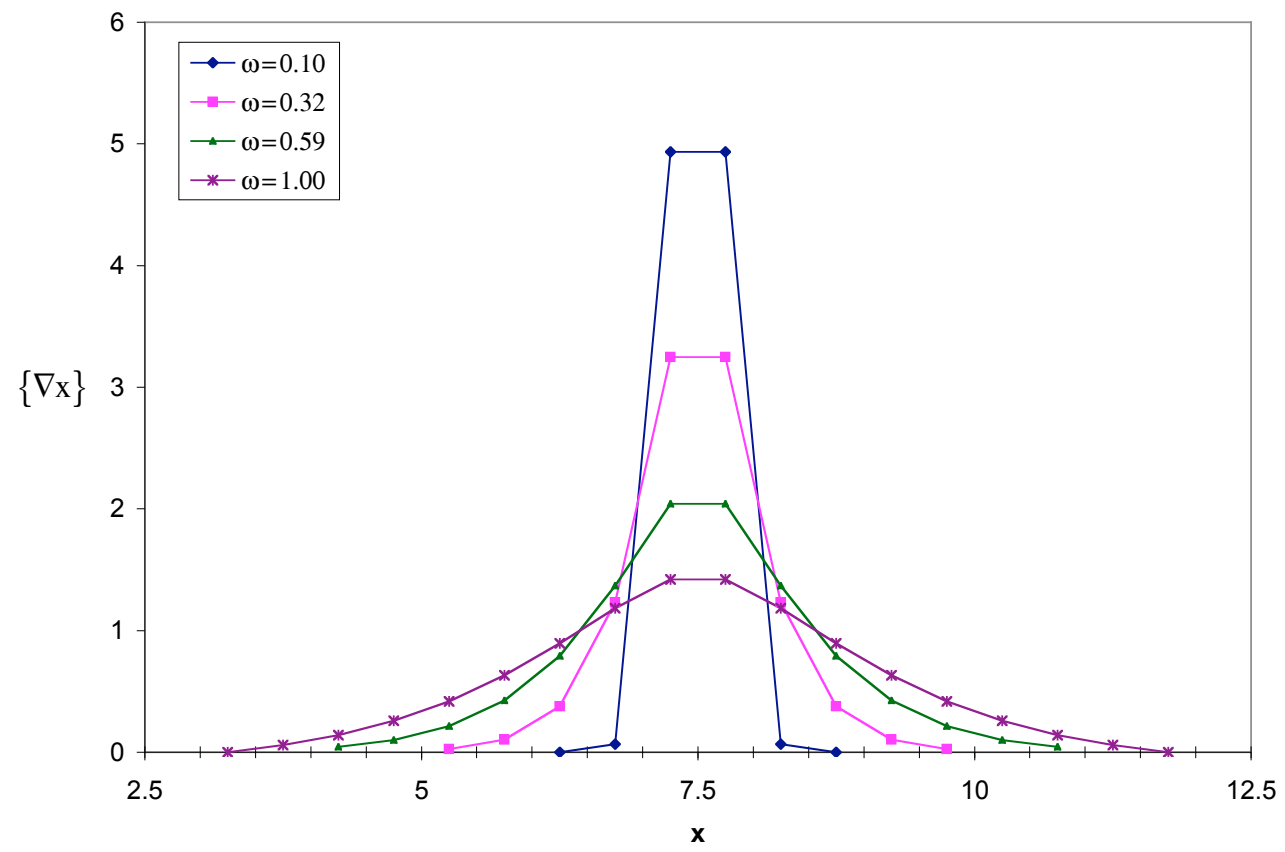

Figure 5.19 - The effect of $\omega$ on $\{\nabla x\}$ of a step-function using $\Theta=\operatorname{Exp}\left(-\frac{|\xi|}{\omega}\right)$.

As $\omega$ decreases, the gradient profile becomes higher and more compact. The curves in Figure 5.19 look very similar to the curves in Figure 5.18. However there is a subtle difference between the two sets of curves as these curves approach 0 . These differences are illustrated in Figure 5.20 where two curves from Figures 5.18 and 5.19 have been plotted together. 


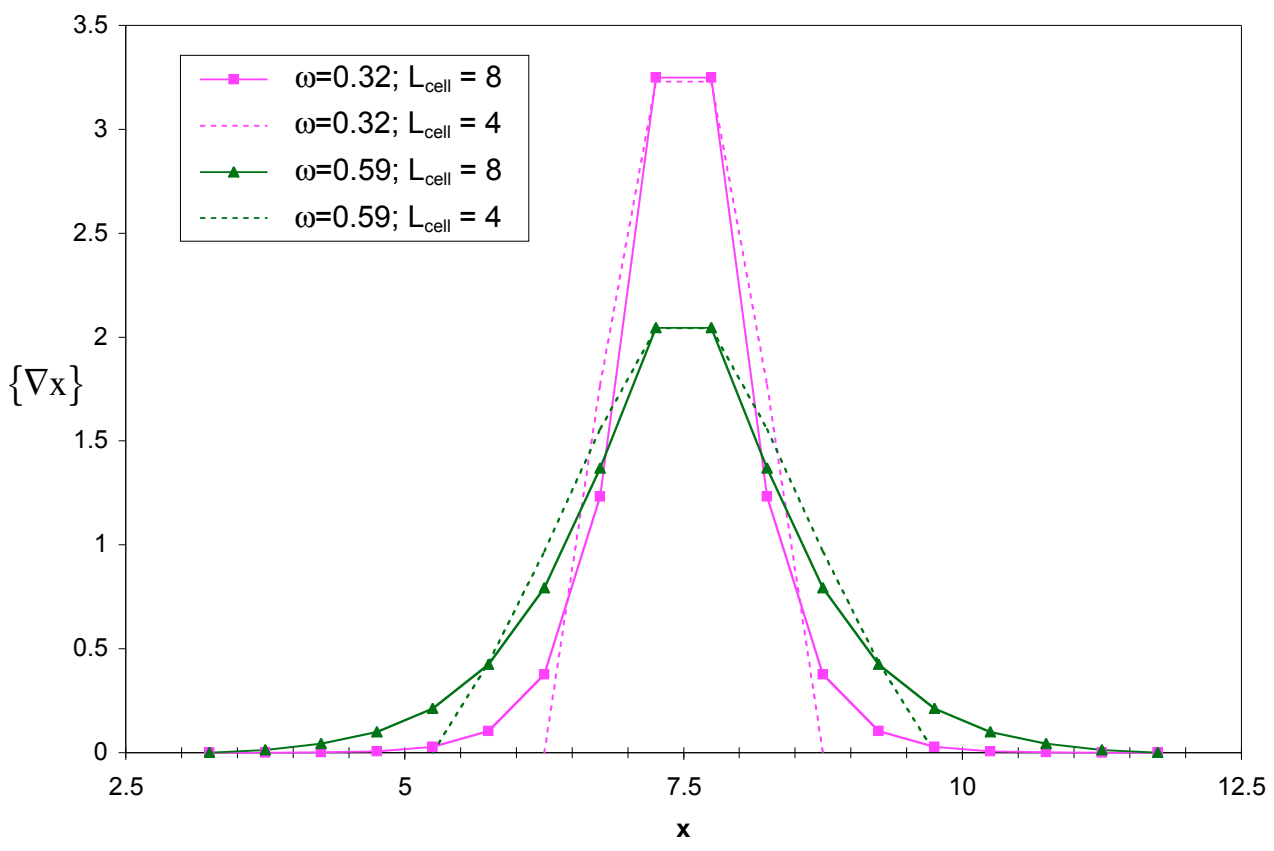

Figure 5.20 - The effect of an $\mathrm{L}_{\text {cell }}$ that is too small for a given $\omega$.

When the integration volume is used to cut off the effect of $\{\nabla x\}$, the tail of the gradient profile is lost. This loss could be significant if $\omega$ was small (resulting in a wide gradient affected zone) and the integration volume was too small to let the gradient profile sufficiently decay. Thus it is very important to check the integration volume size for a given $\omega$ to ensure that gradient profile has adequately decayed.

\subsection{Convergence of Nearest Neighbor Integration Volumes and Large $\omega$ 's}

It is not intuitive that $\{\nabla \mathrm{x}\}$ is independent of $\Theta$ for nearest neighbor integration volumes and that $\{\nabla \mathbf{x}\}$ converges to a non-zero value for large $\omega$ 's. Nor is it intuitive that both of these phenomena have the same root cause: space discretization. The 
following example will illustrate how the grid upon which $\{\nabla \mathbf{x}\}$ is calculated causes these two problems.

Take an arbitrary function $\mathrm{y}=f(\mathrm{x})$ evaluated on a grid with 5 elements each with the same length $\ell$ as shown in Figure 5.21.

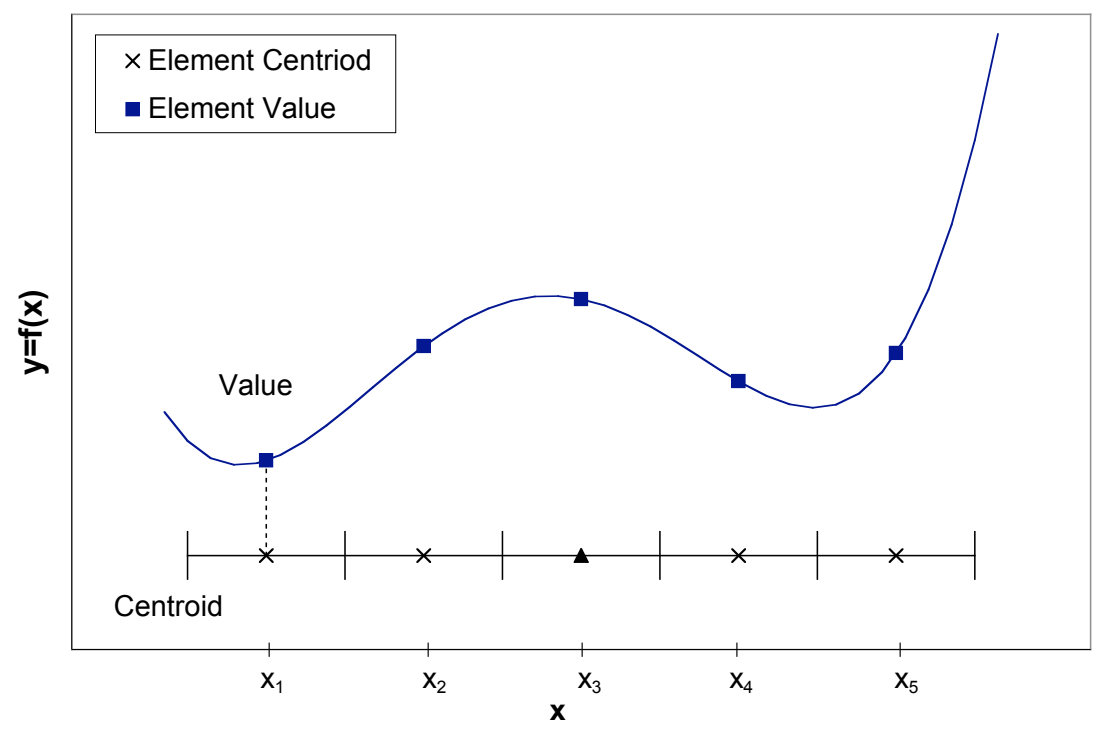

Figure 5.21 - An arbitrary function plotted over a uniform grid composed of 5 elements. $\{\nabla \mathbf{x}\}$ was taken at the element designated by $\boldsymbol{\Lambda}$.

Writing out the summation in Eq. 5.2 that makes up the $\{\nabla x\}$ in Figure 5.21 results in

$$
\begin{array}{r}
\Delta \mathrm{y}_{1-3} \xi_{1-3} \operatorname{Exp}\left(-\frac{\left|\xi_{1-3}\right|}{\omega}\right) \ell_{1}+\Delta \mathrm{y}_{2-3} \xi_{2-3} \operatorname{Exp}\left(-\frac{\left|\xi_{2-3}\right|}{\omega}\right) \ell_{2}+ \\
\{\nabla \mathrm{x}\} \equiv \frac{\Delta \mathrm{y}_{4-3} \xi_{4-3} \operatorname{Exp}\left(-\frac{\left|\xi_{4-3}\right|}{\omega}\right) \ell_{4}+\Delta \mathrm{y}_{5-3} \xi_{5-3} \operatorname{Exp}\left(-\frac{\left|\xi_{5-3}\right|}{\omega}\right) \ell_{5}}{\left(\xi_{1-3}\right)^{2} \operatorname{Exp}\left(-\frac{\left|\xi_{1-3}\right|}{\omega}\right) \ell_{1}+\left(\xi_{2-3}\right)^{2} \operatorname{Exp}\left(-\frac{\left|\xi_{2-3}\right|}{\omega}\right) \ell_{2}+} \\
\left(\xi_{4-3}\right)^{2} \operatorname{Exp}\left(-\frac{\left|\xi_{4-3}\right|}{\omega}\right) \ell_{4}+\left(\xi_{5-3}\right)^{2} \operatorname{Exp}\left(-\frac{\left|\xi_{5-3}\right|}{\omega}\right) \ell_{5}
\end{array}
$$


where $\Delta \mathrm{y}_{\mathrm{i}-\mathrm{j}}=f\left(\mathrm{x}_{\mathrm{i}}\right)-f\left(\mathrm{x}_{\mathrm{j}}\right), \xi_{\mathrm{i}-\mathrm{j}}=\mathrm{x}_{\mathrm{i}}-\mathrm{x}_{\mathrm{j}}$, and $\ell_{\mathrm{i}}=$ length of element i. Since each element has the same length, then the following simplifications can be made:

- $\ell_{1}=\ell_{2}=\ell_{3}=\ell_{4}=\ell$

- $\quad \xi_{3-5}=-\xi_{3-1}, \xi_{3-4}=-\xi_{3-2}$

Thus Eq. (5.4) simplifies to

$$
\{\nabla \mathrm{x}\}=\frac{\ell\left(\left[\Delta \mathrm{y}_{5-3}-\Delta \mathrm{y}_{1-3}\right] \xi_{1-3} \operatorname{Exp}\left(-\frac{\xi_{1-3}}{\omega}\right)+\left[\Delta \mathrm{y}_{4-3}-\Delta \mathrm{y}_{2-3}\right] \xi_{2-3} \operatorname{Exp}\left(-\frac{\xi_{2-3}}{\omega}\right)\right)}{2 \ell\left(\left[\xi_{1-3}\right]^{2} \operatorname{Exp}\left(-\frac{\xi_{1-3}}{\omega}\right)+\left[\xi_{2-3}\right]^{2} \operatorname{Exp}\left(-\frac{\xi_{2-3}}{\omega}\right)\right)}
$$

When $\omega$ is very small the damping rate is very steep. If the mesh is not refined enough, then one exponential term will dominate the summation. In this example, $\operatorname{Exp}\left(-\frac{\xi_{2-3}}{\omega}\right)>\operatorname{Exp}\left(-\frac{\xi_{1-3}}{\omega}\right)$ meaning Eq. (5.5) then further simplifies to

$$
\{\nabla \mathrm{x}\}=\frac{\left[\Delta \mathrm{y}_{4-3}-\Delta \mathrm{y}_{2-3}\right] \xi_{2-3} \operatorname{Exp}\left(-\frac{\xi_{2-3}}{\omega}\right)}{2\left[\xi_{2-3}\right]^{2} \operatorname{Exp}\left(-\frac{\xi_{2-3}}{\omega}\right)}=\frac{\left[\Delta y_{4-3}-\Delta y_{2-3}\right]}{2 \xi_{2-3}}
$$

The resulting Eq. (5.6) shows that when a large $\omega$ is used on a coarse mesh, $\{\nabla \mathrm{x}\}$ converges to a non-zero value.

Equation (5.6) also represents the summation equation for an integration volume containing only nearest neighbors. Since the two neighbors contained within the integration volume are the same distance from the point at which $\{\nabla x\}$ is taken, the exponential $\Theta$ functions cancel out. Thus $\{\nabla \mathrm{x}\}$ will always be independent of $\Theta$ for nearest neighbor integration volumes. 
The same trends shown in this section for a uniform mesh are observed for a nonuniform mesh. When the derivation of Eq. (5.6) is applied to a non-uniform mesh, one rather than two terms will dominate the summation: the term with the smallest $\xi$ value.

Take for example the function and grid in Figure 5.22.

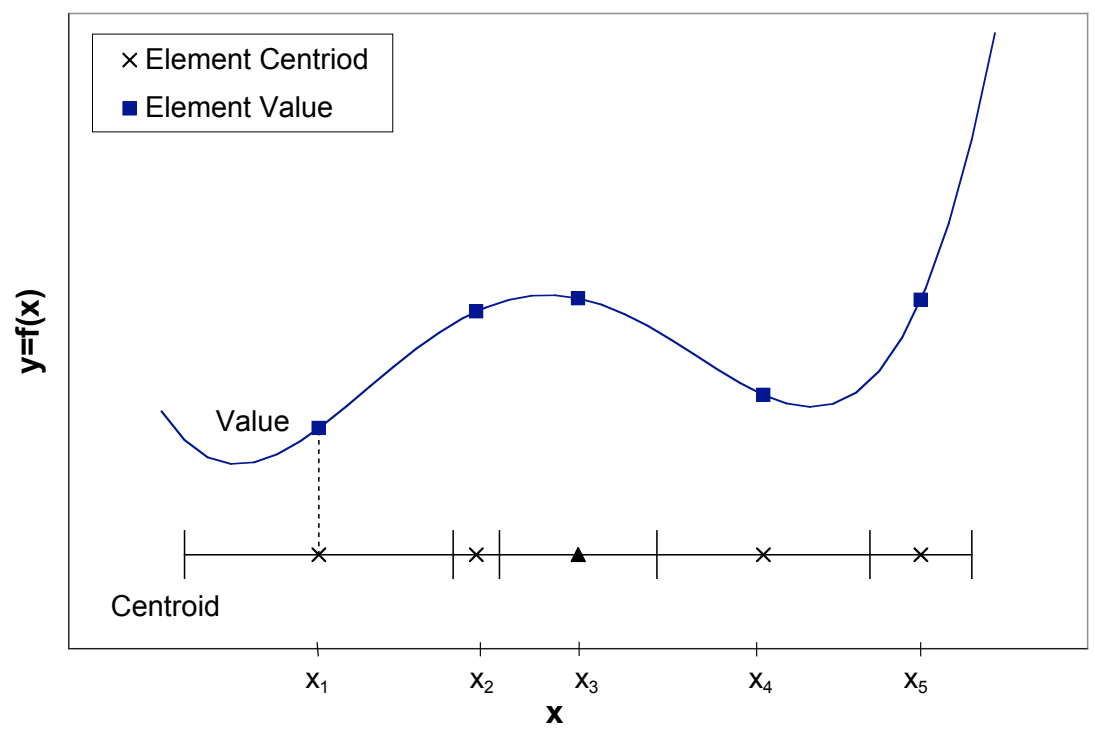

Figure 5.22 - An arbitrary function plotted over a non-uniform grid composed of 5 elements. $\{\nabla \mathbf{x}\}$ was taken at the element designated by $\mathbf{\Delta}$.

For this non-uniform grid, $\operatorname{Exp}\left(-\frac{\xi_{2-3}}{\omega}\right)$ is much larger than any other exponential term because $\xi_{2-3}$ is smaller than any other $\xi$ term. Thus Eq. (5.4) simplifies to

$$
\{\nabla \mathrm{x}\}=\frac{\Delta \mathrm{y}_{2-3} \xi_{2-3} \operatorname{Exp}\left(-\frac{\xi_{2-3}}{\omega}\right)}{\left[\xi_{2-3}\right]^{2} \operatorname{Exp}\left(-\frac{\xi_{2-3}}{\omega}\right)}=\frac{\Delta \mathrm{y}_{2-3}}{\xi_{2-3}}
$$

Again $\{\nabla \mathrm{x}\}$ converges to a non-zero value. 


\section{CHAPTER 6}

\section{APPLICATION OF THE LOCAL AND NON-LOCAL CRYSTAL PLASTICITY MODELS TO POLYCRYSTALLINE COPPER}

In Chapter 6, primarily macroscale results obtained with the local and non-local crystal plasticity models are presented. Both crystal plasticity models were used to simulate the tensile response of polycrystalline copper with various grain sizes in order to investigate the Hall-Petch effect. The simulation results show that the non-local model does predict a grain size dependence of the yield strength. Comparisons with experimental data presented on a Hall-Petch plot reveals that there is qualitative agreement between simulations and the experiments.

\subsection{Simulation Parameters}

\subsubsection{Determination of Grain Orientations}

The polycrystals used in the simulations for this research project contained between 16 and 25 grains. Because 16-25 grains constitute a small statistical population, assigning a random initial orientation to each grain in the polycrystal does not guarantee that the stress-strain response will be representative of a larger polycrystal with random orientations. Thus, 100 different random orientations sets (each set containing 16-25 orientations making up the polycrystal) were generated for each polycrystal. Each 
polycrystal was then deformed with the local crystal plasticity ${ }^{10}$ model resulting in 100 stress-strain curves. The average stress value at $0.2 \%$ strain was computed from these 100 stress-strain curves, and the stress-strain curve that was closest to this average stress value was deemed the "average" stress-strain curve. An example of this procedure for a 25 grain polycrystal is shown in Figure 6.1 .

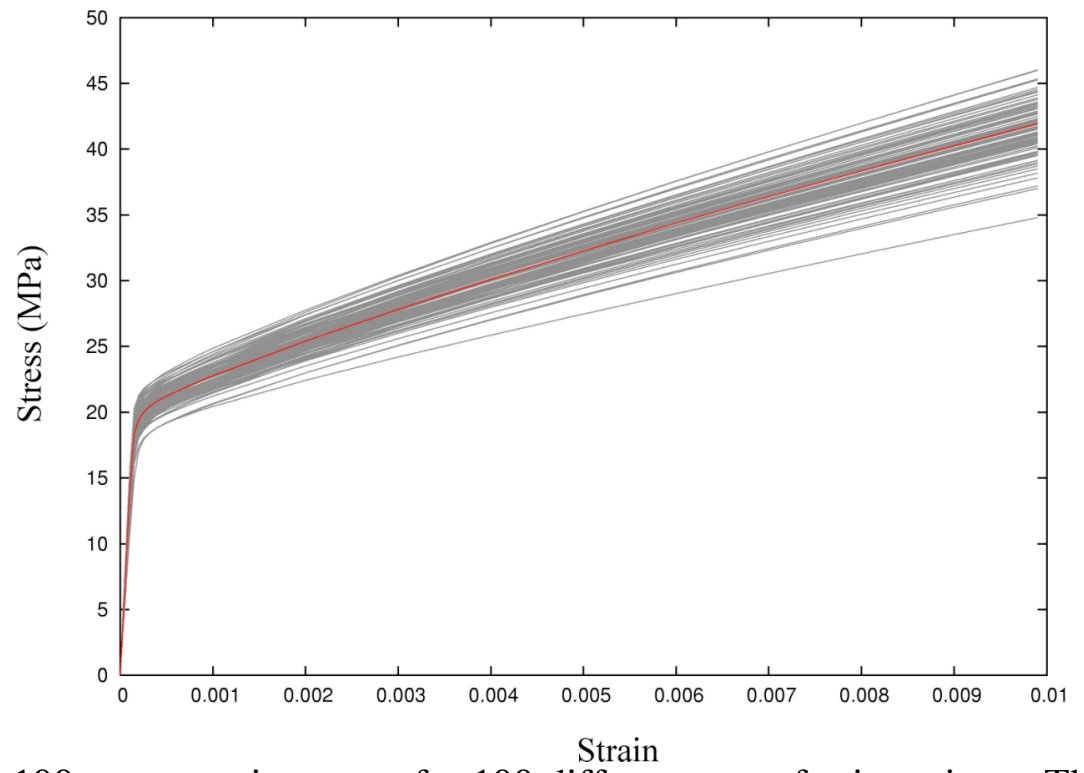

Figure 6.1 - 100 stress-strain curves for 100 different sets of orientations. The "average" stress-strain curve is in red.

The orientation set associated with the "average" stress-strain curve (the red stress-strain curve in Figure 6.1) was then used to represent the microstructure of the 25 grain polycrystal.

\footnotetext{
${ }^{10}$ The local plasticity model used a set of generic model parameters applicable to the material being modeled. It was assumed that average orientation was independent of the model parameters.
} 


\subsubsection{Elastic and Viscoplastic Parameters}

The Burgers vector and anisotropic elastic constants $\left(L_{11}, L_{12}\right.$, and $\left.L_{44}\right)$ for bulk polycrystalline copper were taken from the literature (Hertzberg, 1983). In addition, the two viscoplastic flow rule parameters, $\dot{\gamma}_{0}$ and $\mathrm{m}$, were set to typical literature values. All of these constants are listed in Table 6.1.

Table 6.1 - Elastic and viscoplastic parameters used in copper simulations

\begin{tabular}{|c|c|c|c|c|c|c|}
\hline & $L_{11}(\mathrm{GPa})$ & $L_{12}(\mathrm{GPa})$ & $L_{44}(\mathrm{GPa})$ & $\mathrm{b}(\mathrm{A})$ & $\dot{\gamma}_{0}(1 / \mathrm{sec})$ & $\mathrm{m}$ \\
\hline Copper & 168.4 & 121.4 & 75.4 & 2.556 & 1 & 25 \\
\hline
\end{tabular}

\subsubsection{Hardening Parameters}

The "average" orientation set, determined using the procedure described in Section 6.1.1, was then used to establish the specific hardening parameters for each the experimental data set. Experimental data sets that show the dependence of yield strength on grain size usually have multiple stress-strain curves, one each for a different grain size. The experimental stress-strain curve for the largest grain size was assumed to represent the length scale independent response, i.e. a stress-strain curve for a grain size whose yield strength is grain size independent. The hardening parameters $\tau_{\mathrm{t}=0}^{\mathrm{CSS}}, \mathrm{c}_{\mathrm{KM} 1}$, and $\mathrm{c}_{\mathrm{KM} 2}$ were then derived by fitting Eq. 4.20 to the largest grain size stress-strain curve using the local crystal plasticity model and the previously determined "average" orientation. 
Two data sets that showed the grain size dependence of copper were taken from the literature and used to verify the predicted yield strength results of the non-local model. Because the emphasis of this research project was on the yield strength, the hardening parameters were fit to the low strain region: between 0 and $5 \%$ true strain. In both of these data sets, data in this region was sparse. However it was still possible to obtain a reasonable fit.

The first data set from Fernandes and Viera (2000) contained 5 stress-strain curves from polycrystals with grain sizes ranging from $15 \mu \mathrm{m}-350 \mu \mathrm{m}$ as seen in Figure 6.2a. Unfortunately, the specimen dimensions were not reported. The local crystal plasticity model was used to fit the hardening parameters to the $350 \mu \mathrm{m}$ stress-strain curve. The predicted stress-strain curve using the best-fit hardening parameters (tabulated in Table 6.2) is shown in Figure 6.2b. The other data set, from Hansen (1979), contained 3 stress-strain curves from polycrystals with grain sizes ranging from $14 \mu \mathrm{m}$ $220 \mu \mathrm{m}$ as seen in Figure 6.3a. Unfortunately, the specimen dimensions were not reported for this data set either. The predicted stress-strain curve using the best-fit hardening parameters (tabulated in Table 6.2) is shown in Figure 6.3b. 


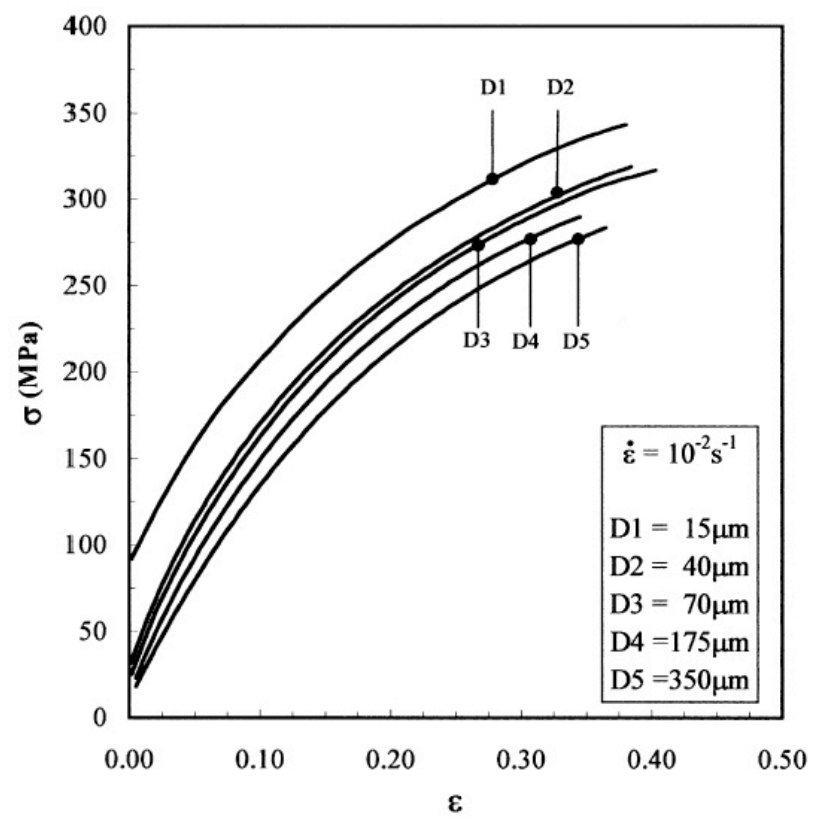

(a) Experimental

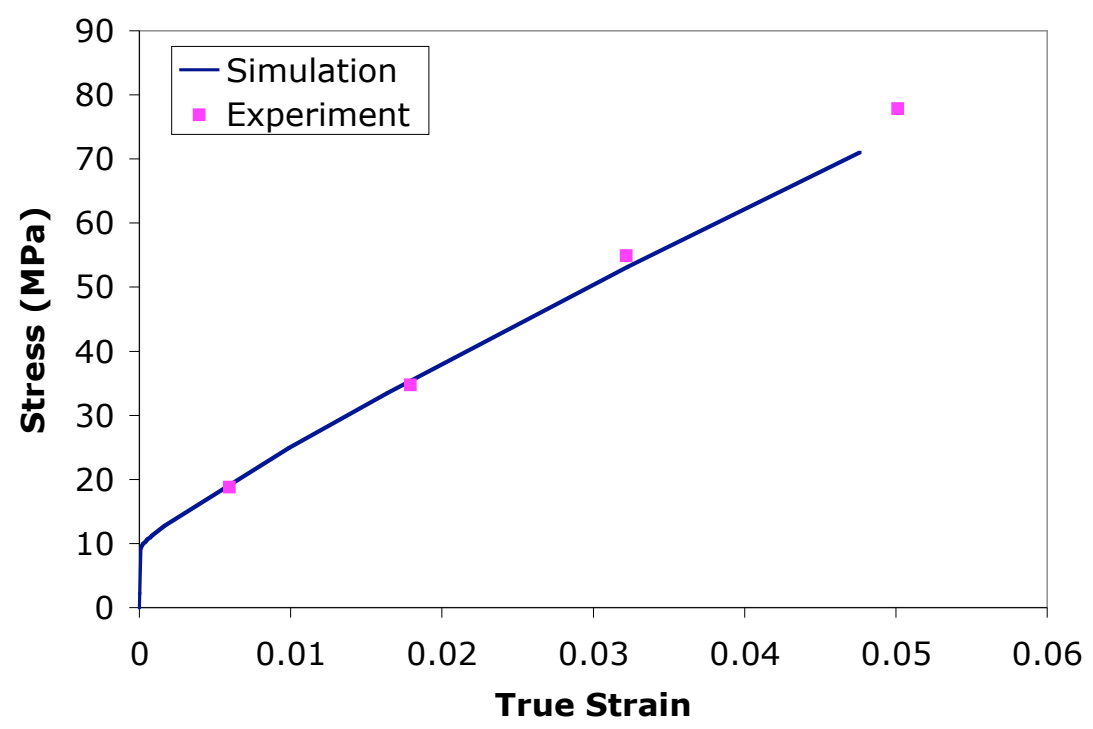

(b) Local model fit to the $350 \mu \mathrm{m}$ curve

Figure 6.2 -Stress-strain curves from Fernandes and Viera (2000) 


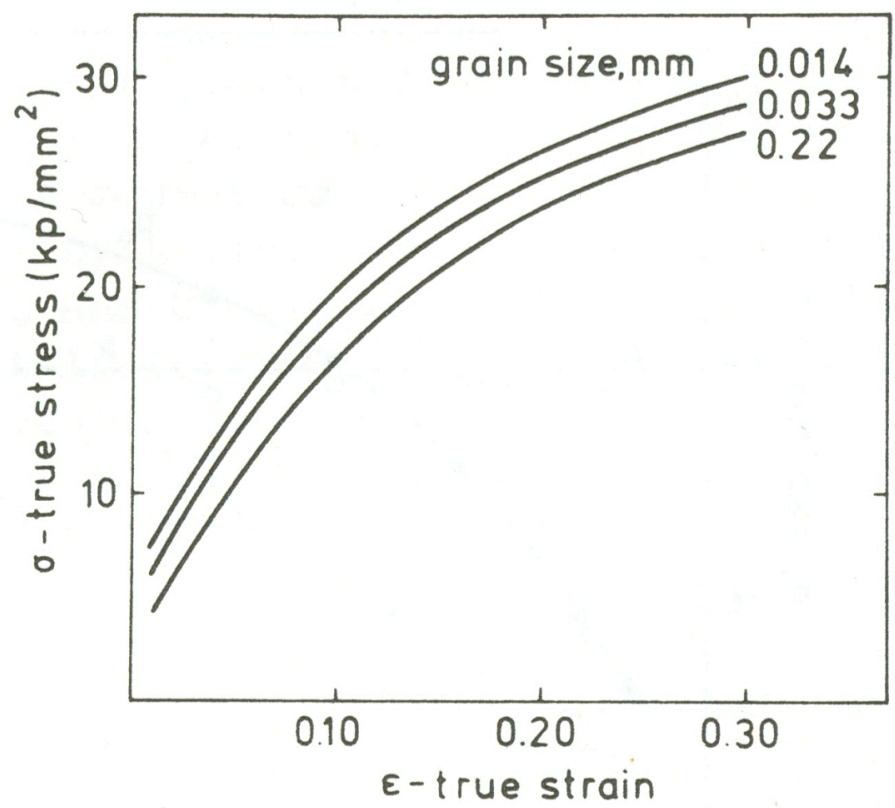

(a) Experimental

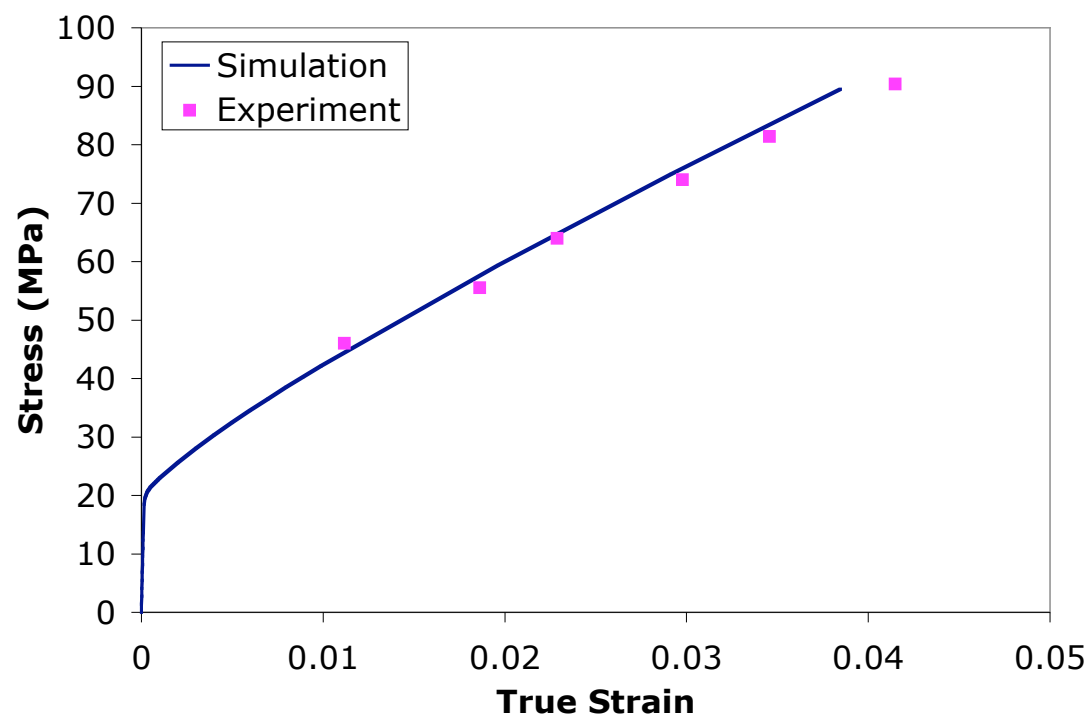

(b) Local model fit to the $220 \mu \mathrm{m}$ curve

Figure 6.3 -Stress-strain curves from Hansen (1979) 
Table 6.2 contains the hardening parameters used in all the copper simulations.

Table 6.2 - Hardening parameters used in copper simulations

\begin{tabular}{|c|c|c|c|c|}
\hline & Grain Size & $\tau_{\mathrm{t}=0}^{\mathrm{CSS}}(\mathrm{MPa})$ & $\mathrm{c}_{\mathrm{KM} 1}(1 / \mathrm{m})$ & $\mathrm{c}_{\mathrm{KM} 2}$ \\
\hline Fernandes and Viera & $350 \mu \mathrm{m}$ & 3.50 & $19.948 \times 10^{8}$ & 18.0 \\
\hline Hansen & $220 \mu \mathrm{m}$ & 7.56 & $9.397 \times 10^{8}$ & 23.9 \\
\hline
\end{tabular}

\subsubsection{Determination of Non-Local Model Parameters}

At this point all the parameters needed to run the local model have been determined. However, the non-local model requires 2 additional constants: $\mathrm{C}$ and $\omega$. $\mathrm{C}$ is important because it affects $\rho_{\mathrm{t}=0}^{\mathrm{SSD}}$ for a given $\tau_{\mathrm{t}=0}^{\mathrm{CSS}}$ via the Taylor equation. As C increases, $\rho_{\mathrm{t}=0}^{\mathrm{SSD}}$ decreases and vice versa. It is generally accepted that $\mathrm{C}$ should be between 0 and 1 .

The variable $\omega$ affects $\rho^{\mathrm{GND}}$ through the $\Theta$ function in $\{\tilde{\mathbf{G}}\}$ and $\{\hat{\mathbf{G}}\}$. In Section 5.2.2, it was demonstrated that a larger $\omega$ resulted in a higher $\rho^{\mathrm{GND}}$ at the grain boundary but a smaller $\rho^{\mathrm{GND}}$ affected zone around the grain boundary. This $\rho^{\mathrm{GND}}$ affected zone around the grain boundary is considered the grain boundary hardened region.

Experiments suggest that the width of the grain boundary hardened region is in the range of 3-5 $\mu \mathrm{m}$ across the grain boundary (Soifer et al., 2002; Soer et al., 2005).

Unfortunately, it is not possible to predefine a hardened grain boundary width with $\omega$ 
since the width of the hardened grain boundary material depends on a number of variables, including the misorientation between the two grains, $\mathrm{C}$, and $\omega$.

As it turns out, $\omega$ and $\mathrm{C}$ control the slope of the Hall-Petch response (see Section 6.4.2 for details). Thus $\mathrm{C}$ and $\omega$ were fit to the experimental data in the following way.

- Start with $\mathrm{C}=0.3$ and fit the $\omega$ value to the yield strength of the median grain size stress-strain curve in the data set.

- Run a few other grain sizes and check the simulated Hall-Petch slope.

- If the simulated Hall-Petch slope did not match the experimental slope, then C and $\omega$ were adjusted and the material parameters were re-fit to the median grain size.

The last step was repeated until the simulated Hall-Petch response matched the experimental Hall-Petch response.

The primary reason the non-local model was fit to the yield strength rather than the stress-strain curve is the lack of experimental stress-strain data in the yield strength region. $\sigma_{\mathrm{y}}$ in the simulations was defined at $0.2 \%$ offset strain. Yet, the first data point in the Fernandes and Viera (2000) data set was at 0.3\% strain and in the Hansen (1979) data set $1.1 \%$ strain. With no experimental data to fit, yield strength data were used to fit the non-local parameters $\mathrm{C}$ and $\omega$. It has been shown that $\mathrm{C}$ is related to the geometry of the dislocation field. In this model, $\mathrm{C}$ is an adjustable parameter that was kept between 0 and 1. Table 6.3 shows the non-local parameters used in most non-local simulations. 
Table 6.3 - Non-local parameters used in copper simulations

\begin{tabular}{|c|c|c|}
\hline & $\mathrm{C}$ & $\omega(\mu \mathrm{m})$ \\
\hline Fernandes and Viera & 0.8 & 0.60 \\
\hline Hansen & 0.3 & 0.45 \\
\hline
\end{tabular}

Unless otherwise stated, these parameters and the attenuation function $\Theta=\operatorname{Exp}\left(-\frac{|\mathrm{r}|}{\omega}\right)$ were used in the non-local simulations.

\subsubsection{Polycrystalline Material Meshes}

Because experimental data from the literature had no microstructural parameters other than grain size, it was impossible to recreate the exact grain structure of the tested materials. Therefore, a number of different polycrystalline microstructures with different numbers of grains and grain shapes were used in the simulations. Two of the microstructures used idealized grain shapes: square and hexagonal shaped grains. These idealized polycrystals are shown in Figure 6.4. The two rectangular polycrystals containing 24 grains were always deformed along the direction containing 6 grains.

Since the polycrystal with 16 hexagonal-grains was square shaped, it could be deformed in either direction. 


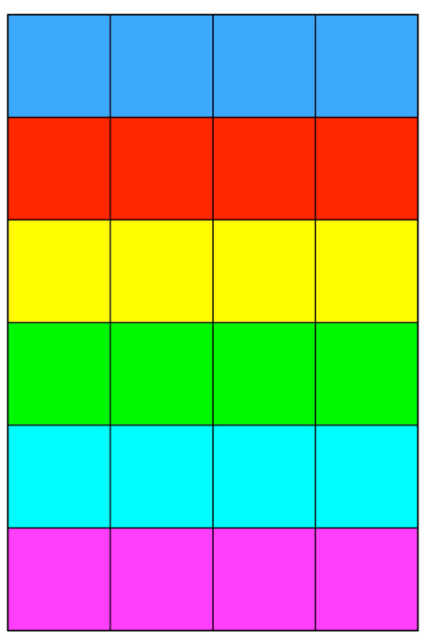

(a) 24 square grains

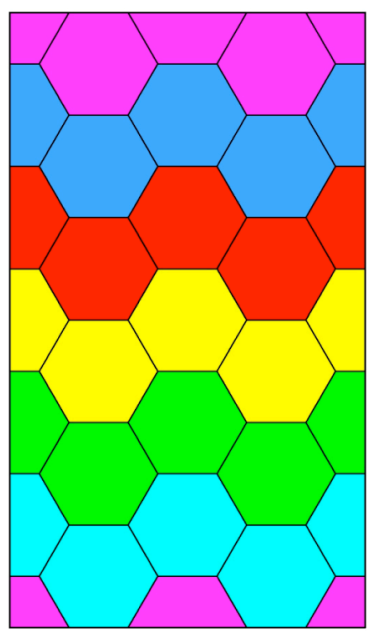

(c) 24 hexagonal grains

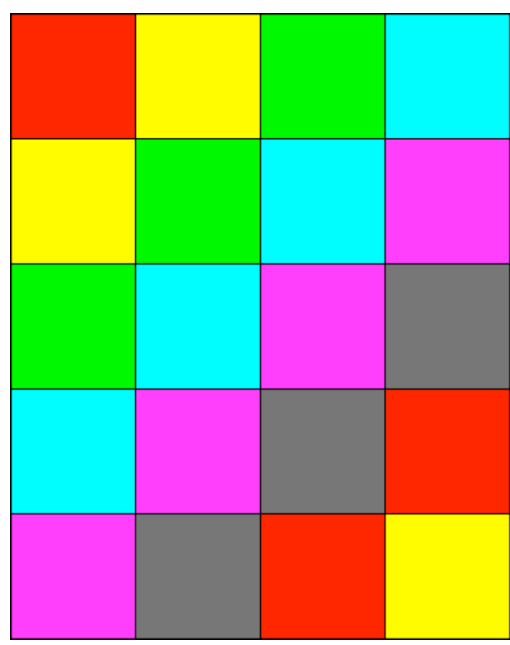

(b) 20 square grains

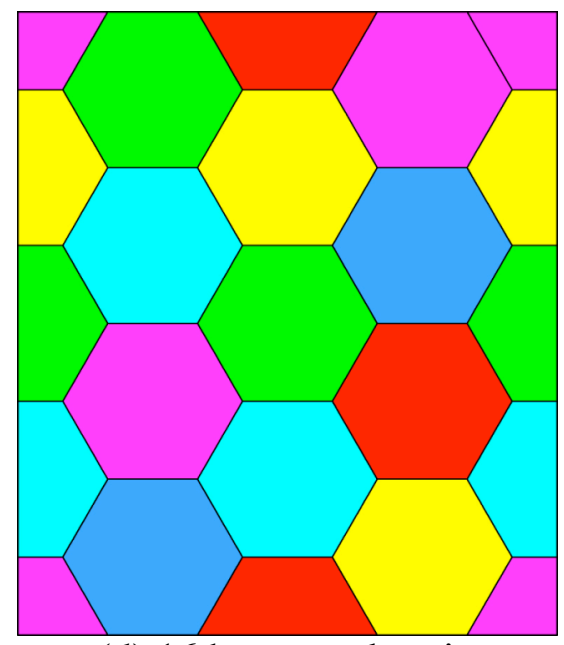

(d) 16 hexagonal grains

Figure 6.4 - Polycrystalline meshes using idealized grain shapes. 
A fourth mesh, representing a realistic looking microstructure, was also used. This polycrystal was square shaped and contained 25 grains as seen in Figure 6.5.

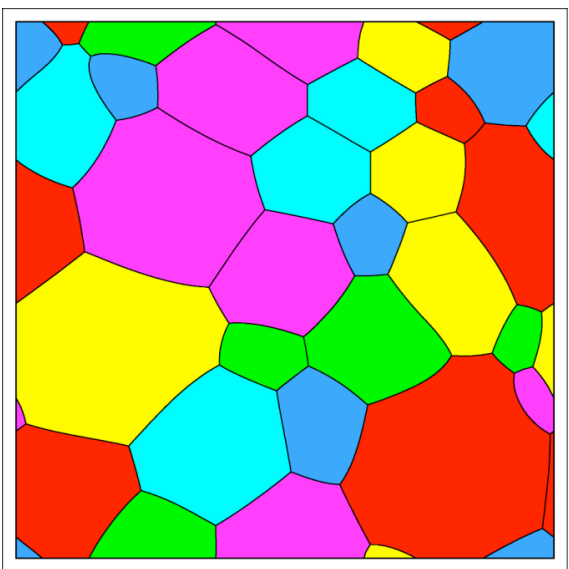

Figure 6.5 - A polycrystalline mesh with 25 realistic grains.

All of the above meshes were $2 \mathrm{D}+1$, which means it is only one element thick in the third dimension. The configuration of each mesh allowed the use of periodic boundary conditions (PBC).

Each mesh was made up of eight node (hexahedral) 3-D isoparametric elements with a single integration point at the element centroid. The number of elements in each mesh ranged from 7,000 to 47,000 depending on the resolution necessary for the nonlocal integral approximation to capture the gradients accurately. When $\Delta \sigma_{\mathrm{y}}$ between two different meshes for the same grain size was less than $1 \%$, the result was considered converged. 


\subsubsection{Boundary Conditions}

Each simulation in this study modeled a displacement controlled tensile test. A positive displacement was applied in one direction and a negative displacement was applied in the opposite direction as shown in Figure 6.6.

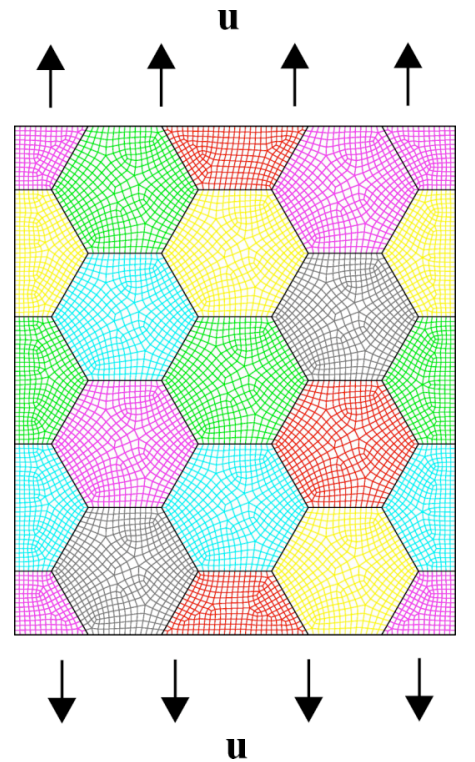

Figure 6.6 - Displacement boundary conditions used in all simulations.

Because the applied displacements were balanced, the material body was in equilibrium. The applied displacements were constrained to the y direction. Thus the top and bottom plane could only move in the y direction. There was no applied displacement or force on either of the sides, which allowed the sides to deform freely.

In most of the simulations, PBC were used. The advantage of using PBC is that it eliminates the need to define the boundary conditions on $\left\{\operatorname{Curl} \mathbf{R}_{\mathbf{h}}^{\mathbf{e}^{-1}}\right\}$ and $\left\{\operatorname{Curl} \mathbf{F}^{\mathbf{P}}\right\}$. On the few simulations that were run without PBC, a no flux boundary condition was applied 
to all surfaces in order to determine the non-local quantities. Numerically, two different versions of such a boundary condition were defined. In the first, the integration volume at surfaces is reduced and non-symmetric, and in the other ghost elements are introduced into the integration volume, which keeps the integration volume symmetric. Because the forces always balanced, there was no need to constrain any nodes or node sets to prevent rigid body motion.

\subsubsection{Free Surface Boundary Condition \#1 (FSBC \#1)}

In the FSBC \#1 approach, the mesh boundary serves as a boundary to the integration volume. An integration volume for an element at a surface using FSBC\#1 is illustrated in Figure 6.7.

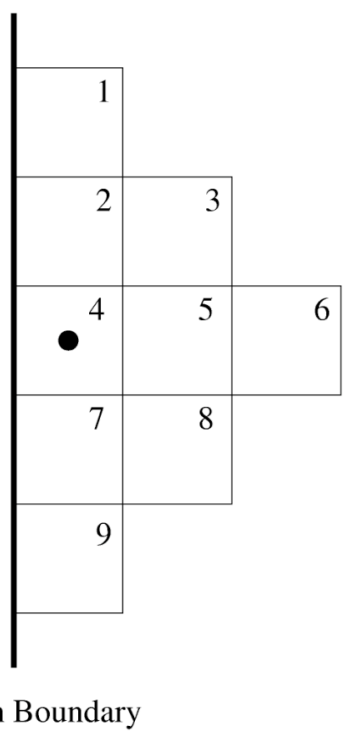

Figure 6.7 - The integration volume for an element at the boundary using FSBC \#1. Non-local quantities are evaluated at the element with $a \bullet$. 
As a result, the integration volume is reduced and no longer symmetric around the element where $\left\{\operatorname{Curl} \mathbf{R}_{\mathbf{h}}^{\mathbf{e}^{-1}}\right\}$ and $\left\{\operatorname{Curl} \mathbf{F}^{\mathbf{P}}\right\}$ are evaluated.

\section{$\underline{\text { 6.1.6.2 Free Surface Boundary Condition \#2 (FSBC \#2) }}$}

In the FSBC \#2 approach, ghost elements are introduced in the integration volume in order to keep integration volume symmetric around the element where $\left\{\operatorname{Curl} \mathbf{R}_{\mathbf{h}}^{\mathbf{e}^{-1}}\right\}$ and $\left\{\right.$ Curl $\left.\mathbf{F}^{\mathbf{P}}\right\}$ are calculated. An integration volume for an element at a surface using FSBC\#2 is illustrated in Figure 6.8.

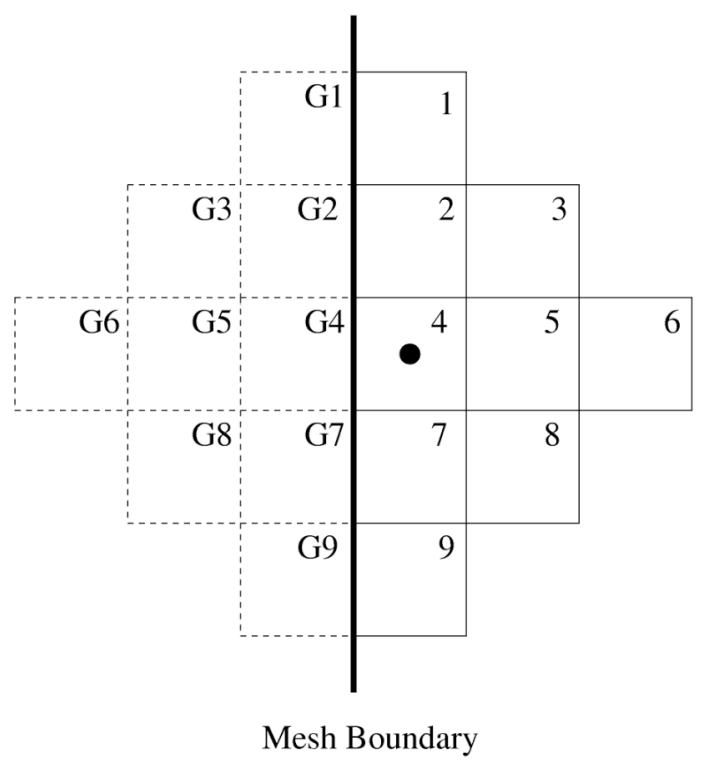

Figure 6.8 - The integration volume for an element at the boundary using FSBC \#2. Non-local quantities are evaluated at the element with $a \bullet$.

The ghost elements were generated by making a reflection of the mesh at the mesh boundary. For the case in Figure 6.8, 9 ghost elements (G1-G9) were created. These 
ghost elements were the same size and shape as that of its reflected element. The value of $\mathbf{R}_{h}^{\mathbf{e}}$ and $\mathbf{F}^{\mathbf{P}}$ assigned to each ghost element is the value of $\mathbf{R}_{h}^{\mathbf{e}}$ and $\mathbf{F}^{\mathbf{P}}$ at the element with $\mathrm{a} \bullet$

\subsubsection{Determination of Yield Strength and Grain Size}

The simulation yield strengths were determined using the $0.2 \%$ offset strain method. Experimentalists commonly use this method to determine experimental yield strengths making yield strength comparisons between simulations and experiment straightforward.

The grain size calculation in the simulations was not complex. The grain size for each grain was simply the square root of the grain surface area. Such a definition was possible because the polycrystal meshes were essentially 2D. Since the size and shape of every grain in the simulation is known, the average grain size was computed as an average over all the grains. Thus, the average simulation grain size is the average value of a normal distribution. Experimentally, the size and shape of every grain is not known. Thus, the average grain size is determined using quantitative analysis on a representative sample of micrographs. Consequently, the average grain size is the average value of a lognormal distribution. The significance, if any, of these differences is not clear. 


\subsection{Effect of Polycrystal Kinematics}

In Chapter 1, the inability of local plasticity models to predict length scale dependent phenomena was illustrated. This shortcoming has driven the development of non-local plasticity models. However, not all non-local plasticity models are able to predict the grain size dependence of yield strength. Non-local models containing plastic deformation based non-local variables will not capture the Hall-Petch effect, unless these models account for the initial microstructure present in the polycrystal. This point is illustrated in Section 6.2.1 and a detailed discussion on the mechanisms that cause the trends observed in Section 6.2.1 is contained in Section 6.2.2.

\subsubsection{Macroscopic Response}

The non-local crystal plasticity model developed in this research project has two non-local variables. One of these variables, $\{\hat{\mathbf{G}}\}$, was associated with plastic deformation variable via $\left\{\operatorname{Curl} \mathbf{F}^{\mathbf{P}}\right\}$ and the other, $\{\tilde{\mathbf{G}}\}$, was associated with the initial microstructure via $\left\{\right.$ Curl $\left.\mathbf{R}_{\mathbf{h}}^{\mathrm{e}^{-1}}\right\}$. In order to demonstrate the importance of initializing the microstructure, the non-local model was run with and without $\{\tilde{\mathbf{G}}\}$. The 24 hexagonal grain polycrystal (Figure 6.4b) was used to create copper polycrystals with four different grain sizes: $14 \mu \mathrm{m}, 20 \mu \mathrm{m}, 40 \mu \mathrm{m}$, and $70 \mu \mathrm{m}$. The tensile response of each polycrystal was simulated using the hardening parameters from Hansen (see Table 6.2 and 6.3). 
The stress-strain results from the non-local crystal plasticity model with and without $\{\tilde{\mathbf{G}}\}$ are very different. The non-local plasticity model without $\{\tilde{\mathbf{G}}\}$ still has an internal length scale associated with $\{\hat{\mathbf{G}}\}$. Thus this model will predict different stressstrain curves for different grains sizes as seen in Figure 6.9. Problems with these simulation results can be seen when they are compared with typical experimental stressstrain curves for polycrystals with differing grain sizes, like those in Figure 6.10. The simulation results from the model without $\{\tilde{\mathbf{G}}\}$ (Figure 6.9) do show an increase in yield strength as grain sizes decreases; however, the simulation results do not show the same type of grain size dependence seen in the experimental results (Figure 6.10). The simulated stress-strain curves are not stacked on top of each other, but rather curves with different slopes pivot off the same point. 


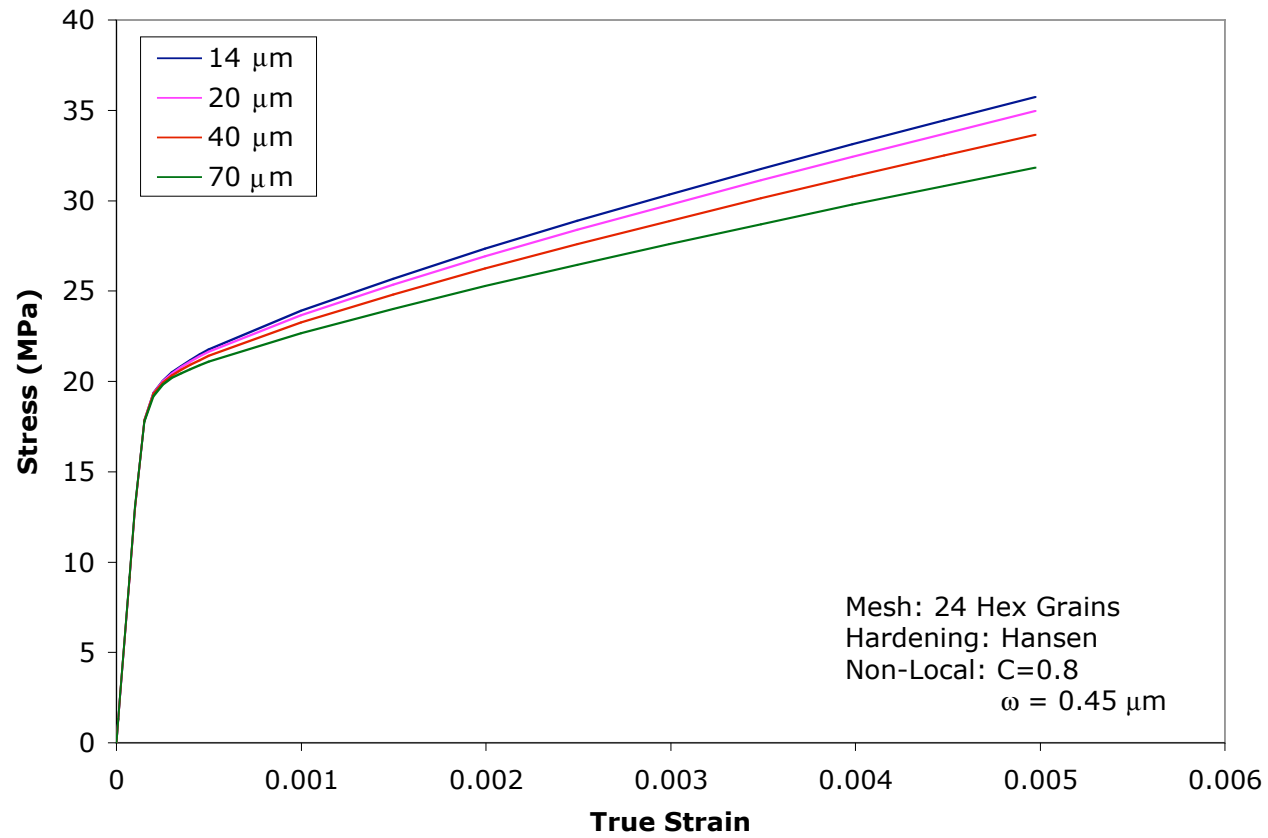

Figure 6.9 - Predicted stress-strain response of polycrystalline copper with grain sizes between $14 \mu \mathrm{m}$ and $77 \mu \mathrm{m}$ from a non-local model without $\{\tilde{\mathbf{G}}\}$.

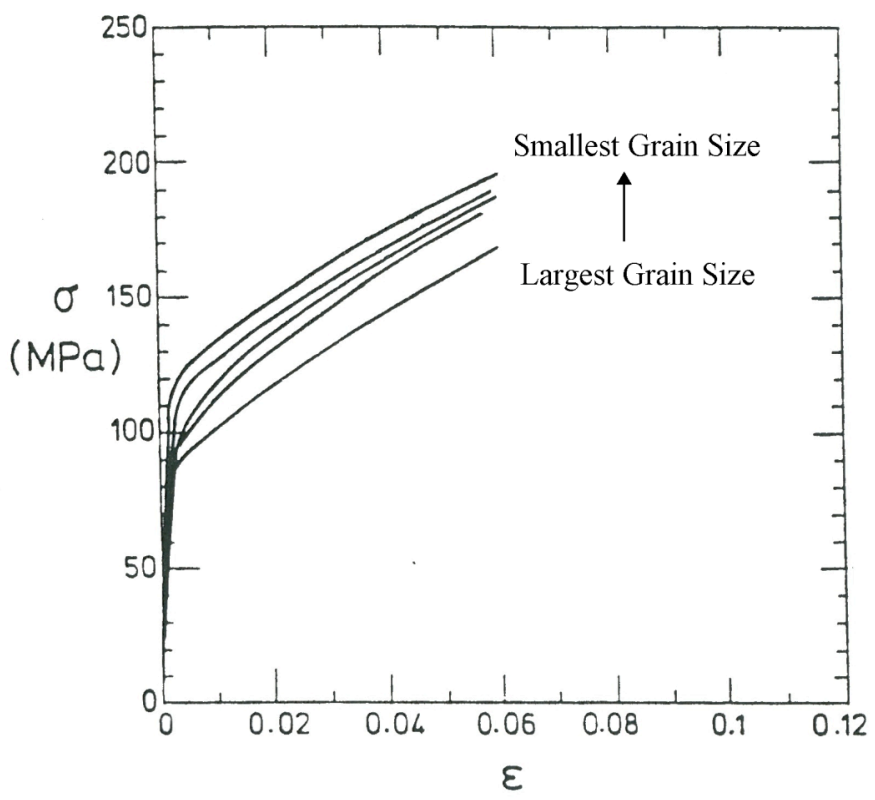

Figure 6.10 - Experimental stress-strain curves for a polycrystalline material with different grain sizes. (Fleck et al., 1994) 
The non-local plasticity model with the $\{\tilde{\mathbf{G}}\}$ term predicts the set of stress-strain curves seen in Figure 6.11.

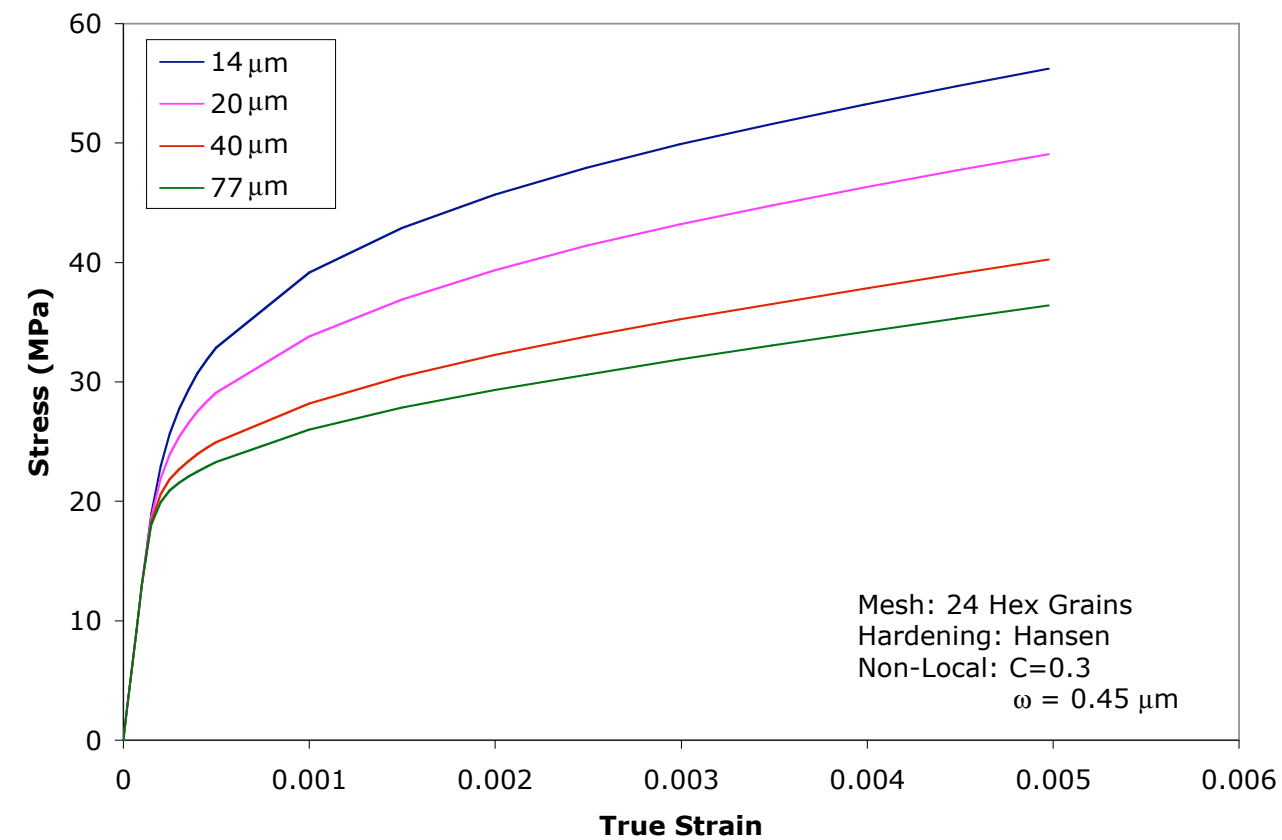

Figure 6.11 - Predicted stress-strain response of polycrystalline copper with grain sizes between $14 \mu \mathrm{m}$ and $77 \mu \mathrm{m}$ from a non-local model with $\{\tilde{\mathbf{G}}\}$.

In this case, the stress-strain curves do not all pivot off the same point. Instead, the stress strain curves are stacked on top of each other. Even though it is difficult to see in Figure 6.11 , the rate of work hardening is slightly higher in the material with the smaller grain size compared to the material with the larger grain size due to $\{\hat{\mathbf{G}}\}$. Both of these trends lead to a very definite grain size effect on the yield strength and to a set of stress-strain curves that compare qualitatively much better with experimental results.

A plot of yield strength vs. $\mathrm{d}^{-0.5}$, otherwise referred to as a Hall-Petch plot, further shows the limitations of a non-local model without $\{\tilde{\mathbf{G}}\}$. The yield strengths for the 4 
grain sizes $(14 \mu \mathrm{m}, 20 \mu \mathrm{m}, 40 \mu \mathrm{m}$, and $70 \mu \mathrm{m})$ calculated by both models are compared with experimental data for copper from Hansen (1983) in Figure 6.12.

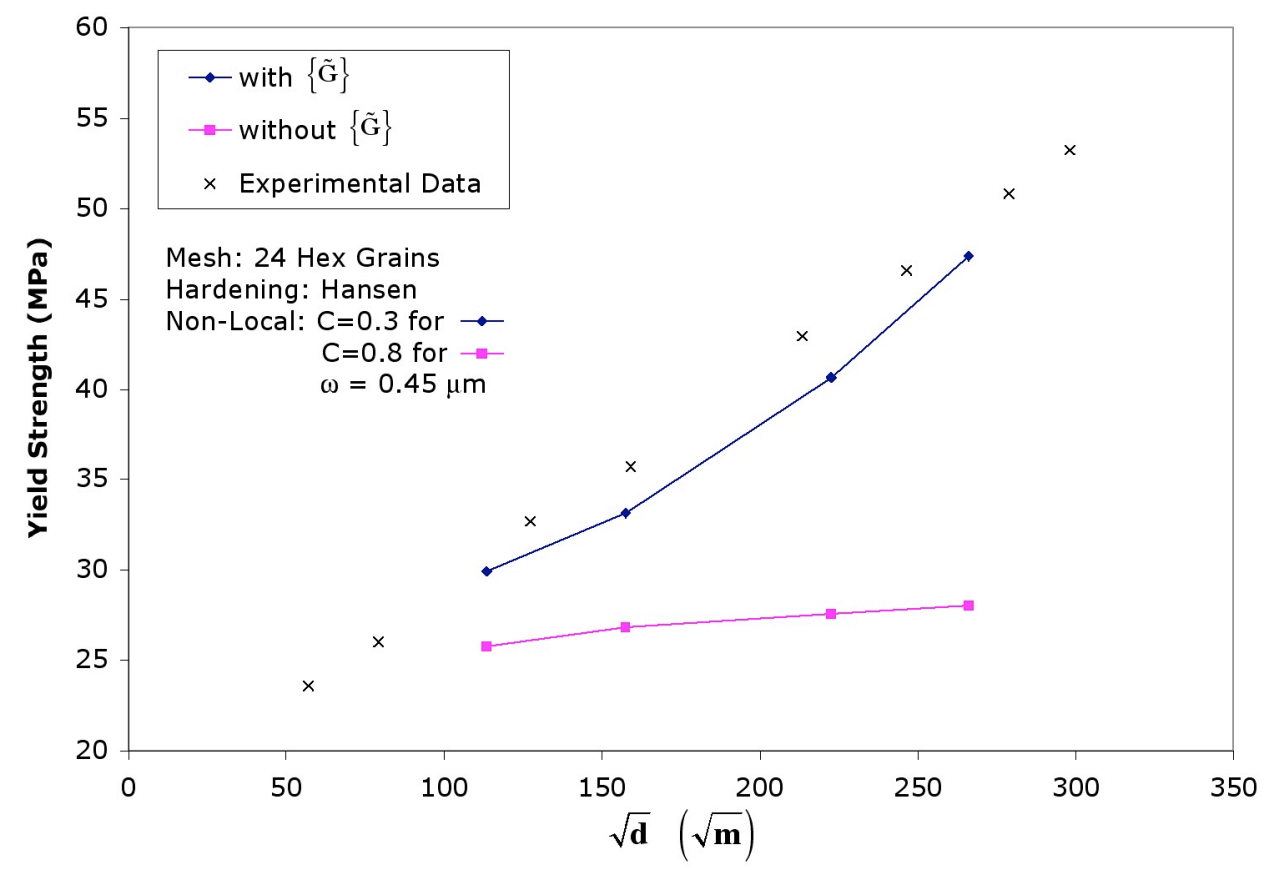

Figure 6.12 - A Hall-Petch plot comparing experimental results to simulation results from the non-local mode with and without $\{\tilde{\mathbf{G}}\}$.

The model without the microstructure initialization predicts very little (2.3 MPa) difference in the yield strength for polycrystals with grain sizes of $14 \mu \mathrm{m}$ and $70 \mu \mathrm{m}$. On the other hand, the model with initialization predicts a significant difference in the yield strength $(17.4 \mathrm{MPa})$ for polycrystals with grain sizes of $14 \mu \mathrm{m}$ and $70 \mu \mathrm{m}$. Here again, the Hall-Petch results from the model with initialization compare qualitatively much better with experimental results. 


\subsubsection{Microscale Response}

The differing macroscale responses presented in Section 6.2.1 are a direct result of differences the model predicts at the microscale. Because the microscale hardening variables do not change in the elastic regime, the initial states of these variables determine the yield strength of the material and therefore govern the Hall-Petch behavior. In this discussion, the variable $\bar{\tau}^{\mathrm{CSS}}$ is introduced to quantify the hardening of the 12 slip systems at each point with a single value. $\bar{\tau}^{\mathrm{CSS}}$ is simply the average flow stress of the 12 slip systems. This discussion also differentiates two different length scales. There are length scales associated with non-local variables and there is a material length scale, which is the one non-local variable length scale that dominates the material's response.

In the non-local model without $\{\tilde{\mathbf{G}}\}$, there is only one non-local variable, $\{\hat{\mathbf{G}}\}$, and therefore only one length scale. Thus, the length scale associated with $\{\hat{\mathbf{G}}\}$ is the material length scale. Since $\{\hat{\mathbf{G}}\}$ is a function of $\mathbf{F}^{\mathbf{P}}$, the length scale in the model is also a function of $\mathbf{F}^{\mathbf{P}}$. At $\varepsilon=0, \mathbf{F}^{\mathbf{P}}=\mathbf{I}$ throughout the material body resulting in $\{\hat{\mathbf{G}}\}=\hat{\mathbf{G}}_{\mathrm{t}=0}^{\text {Tot }}=\mathbf{0}$. Thus the initial $\bar{\tau}^{\mathrm{CSS}}$ state is uniform, independent of grain size, and due solely to $\rho^{\mathrm{SSD}}$ as seen in Figure 6.13. Because initial state of each polycrystal is the same, there is no material length scale associated the polycrystals at $\varepsilon=0$. As plastic deformation evolves, $\mathbf{F}^{\mathbf{P}}$ is no longer uniform throughout the material resulting in a $\bar{\tau}^{\mathrm{CSS}}$ state that evolves differently for different grain sizes as seen in Figure 6.14. 


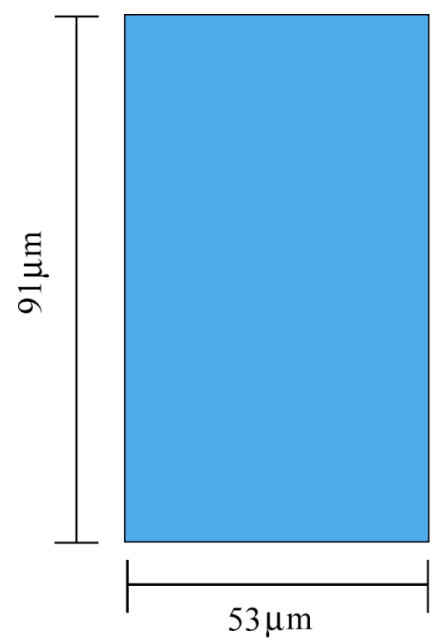

(a) $14 \mu \mathrm{m}$ grain size

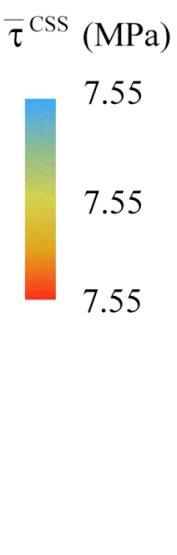

Figure $6.13-\bar{\tau}^{\text {CSS }}$ at $\varepsilon=0$ for a non-local model without $\{\tilde{\mathbf{G}}\}$. Note that the $14 \mu \mathrm{m}$ picture has an area that is roughly $1 / 30^{\text {th }}$ of the $77 \mu \mathrm{m}$ picture.

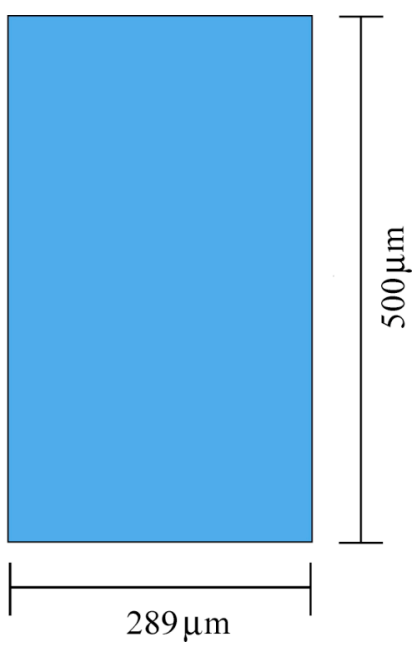

(b) $77 \mu \mathrm{m}$ grain size

\section{急}

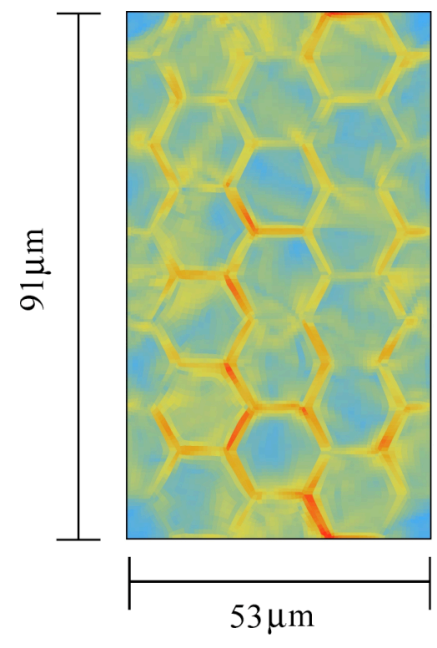

(a) $14 \mu \mathrm{m}$ grain size

$\bar{\tau}^{\mathrm{CSS}}(\mathrm{MPa})$

8.18

12.19

16.21

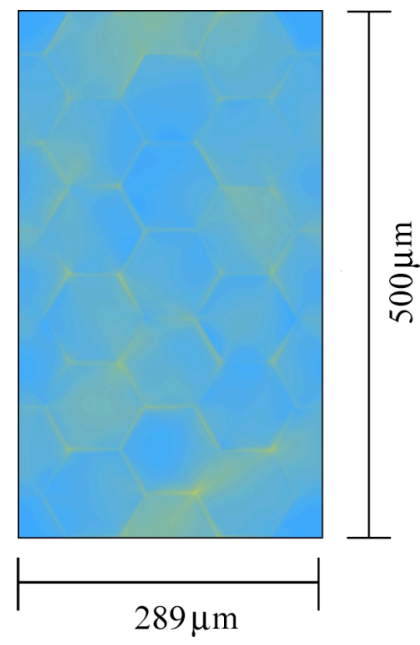

(b) $77 \mu \mathrm{m}$ grain size

Figure $6.14-\bar{\tau}^{\mathrm{CSS}}$ at $\varepsilon=0.5 \%$ for a non-local model without $\{\tilde{\mathbf{G}}\}$. Note that the $14 \mu \mathrm{m}$ picture has an area that is roughly $1 / 30^{\text {th }}$ of the $77 \mu \mathrm{m}$ picture. 
The difference between the polycrystals in Figure 6.14 illustrates that a material length scale is present at $\varepsilon=0.5 \%$.

The problem with the $\{\hat{\mathbf{G}}\}$ based non-local model is that the length scale effects evolve. Initially, there is no material length scale meaning all length scale effects evolve from this point, which is why the stress-strain curves in Figure 6.9 all pivot off the same point. As $\mathbf{F}^{\mathbf{P}}$ evolves the model develops a material length scale that results in an increasingly stronger length scale dependent response. In Figure 6.9, the consequence of this evolving material length scale is faster work hardening rates for polycrystals with smaller grain sizes. Because there is a small change in $\mathbf{F}^{\mathbf{P}}$ at $\varepsilon=0.2 \%$, the grain size dependent work hardening rates result in the small effect of grain size on the yield strength seen in Figure 6.12. Even at $\varepsilon=0.5 \%$, the effect of the material length scale is not large, as evidenced by the $8 \mathrm{MPa}$ difference in minimum and maximum values of $\bar{\tau}^{\mathrm{CSS}}$ in Figure 6.14 and the similar stress-strain curves in Figure 6.9.

In the non-local model with two non-local variables, $\{\tilde{\mathbf{G}}\}$ and $\{\hat{\mathbf{G}}\}$, there are two length scales. In this case, the length scale associated with $\{\tilde{\mathbf{G}}\}$ is a function of $\mathbf{R}_{\mathbf{h}}^{\mathbf{e}}$ and the length scale associated with $\{\hat{\mathbf{G}}\}$ depends on $\mathbf{F}^{\mathbf{P}}$. At $\varepsilon=0 \%, \mathbf{F}^{\mathbf{P}}=\mathbf{I}$ throughout the material, but in this case $\mathbf{R}_{\mathbf{h}}^{\mathbf{e}}$ is not uniform throughout the material making $\hat{\mathbf{G}}_{\mathrm{t}=0}^{\text {Tot }} \neq \mathbf{0}$. Thus the initial $\bar{\tau}^{\mathrm{CSS}}$ state is not uniform as seen in Figure 6.15. 


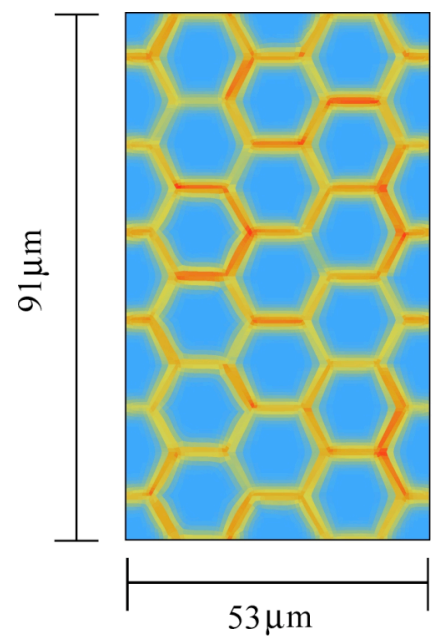

(a) $14 \mu \mathrm{m}$ grain size

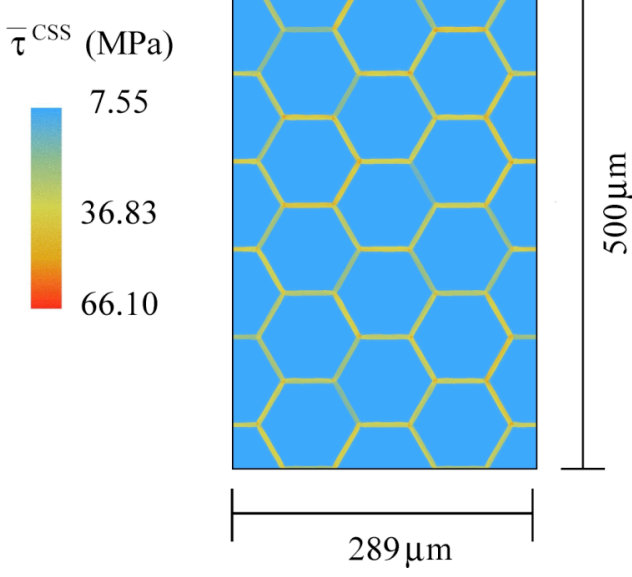

(b) $77 \mu \mathrm{m}$ grain size

Figure $6.15-\bar{\tau}^{\mathrm{CSS}}$ at $\varepsilon=0$ for a non-local model with $\{\tilde{\mathbf{G}}\}$. Note that the $14 \mu \mathrm{m}$ picture has an area that is roughly $1 / 30^{\text {th }}$ of the $77 \mu \mathrm{m}$ picture.

The initial microstructure is made up of interconnected regions of hardened grain boundary material surrounding softer grain interiors. These hardened grain boundary regions result from discontinuities at grain interfaces in the $\mathbf{R}_{\mathbf{h}}^{\mathbf{e}}$ field. Thus there is a material length scale at $\varepsilon=0$ and it is associated with $\{\tilde{\mathbf{G}}\}$. As plastic deformation accumulates, the $\mathbf{F}^{\mathbf{P}}$ field and the length scale associated with $\{\hat{\mathbf{G}}\}$ evolve. Despite the development of a second length scale, the $\bar{\tau}^{\mathrm{CSS}}$ state does not change much as seen in Figure 6.16. 


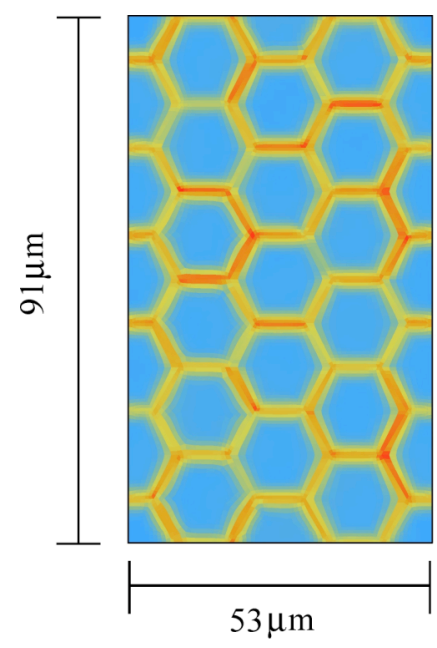

(a) $14 \mu \mathrm{m}$ grain size

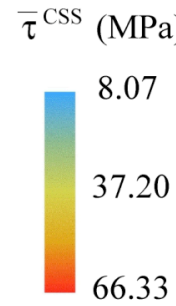

66.33

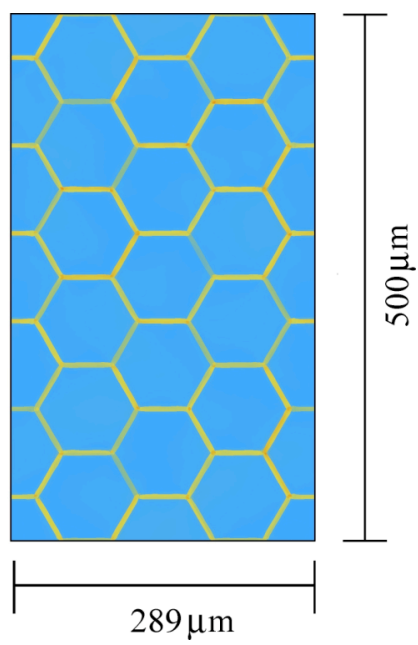

(b) $77 \mu \mathrm{m}$ grain size

Figure $6.16-\bar{\tau}^{\mathrm{CSS}}$ at $\varepsilon=0.5 \%$ for a non-local model with $\{\tilde{\mathbf{G}}\}$. Note that the $14 \mu \mathrm{m}$ picture has an area that is roughly $1 / 30^{\text {th }}$ of the $77 \mu \mathrm{m}$ picture.

The similarity in the $\bar{\tau}^{\mathrm{CSS}}$ states at $\varepsilon=0$ and $\varepsilon=0.5 \%$ shows that the length scale associated with $\{\tilde{\mathbf{G}}\}$ dominates the length scale associated with $\{\hat{\mathbf{G}}\}$ at $\varepsilon=0.5 \%$.

In the non-local model with $\{\tilde{\mathbf{G}}\}$ and $\{\hat{\mathbf{G}}\}$, the material length scale effects do not evolve from a material that has no material length scale. Initially, the material length scale associated with $\mathbf{R}_{\mathrm{h}}^{\mathrm{e}}$ dominates the length scale dependent response starting at $\varepsilon=0$. Therefore the stress-strain curves in Figure 6.11 are stacked on top of each other. The large difference ( $58 \mathrm{MPa}$ ) between the grain boundary hardened regions and the softer grain interiors indicate that the initial length scale effects will be significant and thus the substantial Hall-Petch response seen in Figure 6.12. The length scale associated with $\{\hat{\mathbf{G}}\}$ does evolve with plastic deformation, but its affect on the materials response is still small at $\varepsilon=0.5 \%$. Thus all the stress-strain curves in Figure 6.11 have similar slopes. 
While it is not obvious in Figures 6.15 and $6.16, \bar{\tau}^{\mathrm{CSS}}$ is not constant within the hardened grain boundary region. A plot of $\bar{\tau}^{\mathrm{CSS}}$ vs. distance from the grain boundary shown in Figure 6.17 illustrates how $\bar{\tau}^{\mathrm{CSS}}$ varies within the grain boundary region.

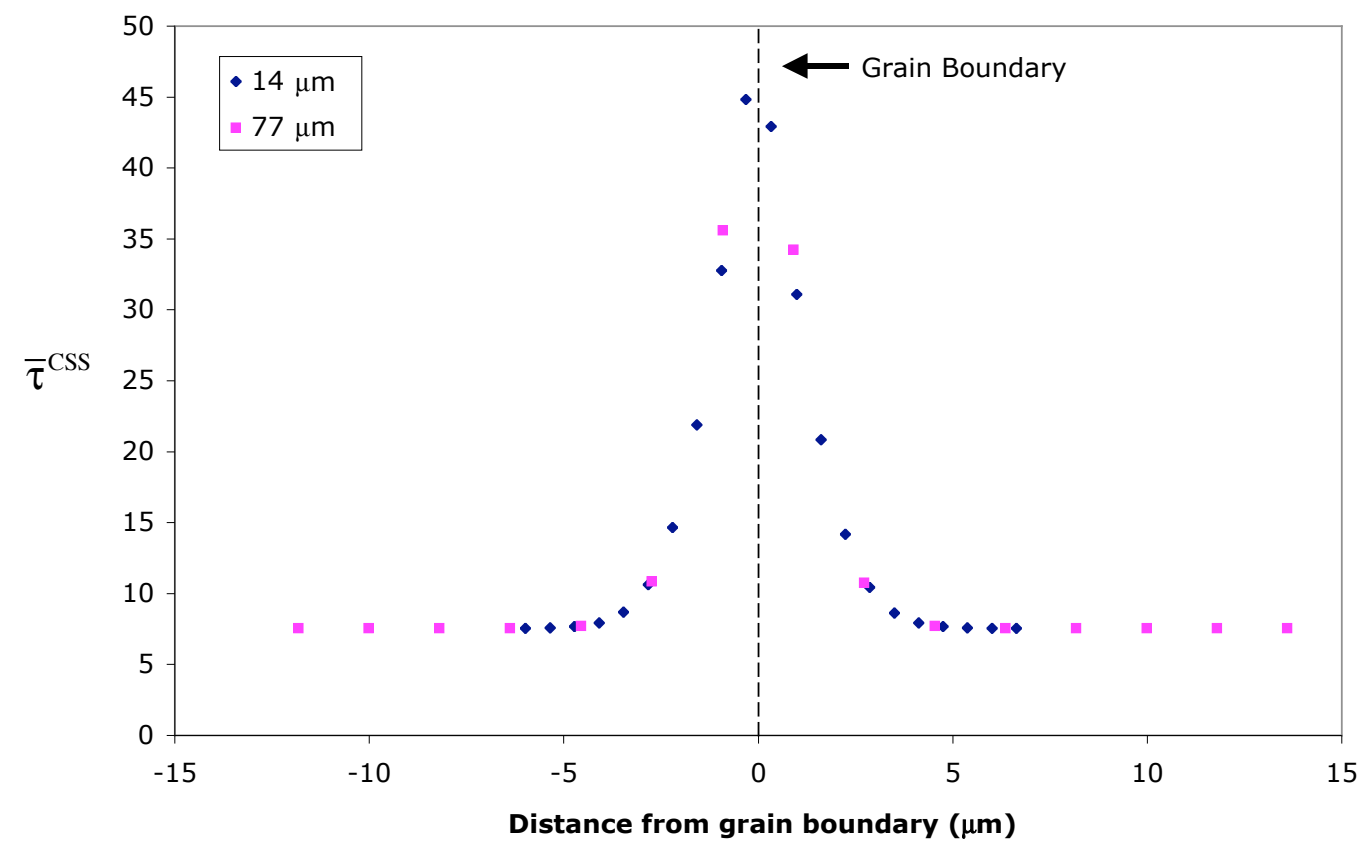

Figure 6.17 - The change in $\bar{\tau}^{\mathrm{CSS}}$ away from the grain boundary in both the $14 \mu \mathrm{m}$ and $77 \mu \mathrm{m}$ grain size polycrystal

First notice that once the $\bar{\tau}^{\mathrm{CSS}}$ results from the $14 \mu \mathrm{m}$ and $77 \mu \mathrm{m}$ grain size polycrystals collapse onto the same curve. This trend demonstrates that the width for similar grain boundary regions is independent of grain size. Also notice that the $\bar{\tau}^{\mathrm{CSS}}$ curve has a maximum value at the interface and decays exponentially (as described by $\Theta$ ) to 7.55 $\mathrm{MPa}$ in the grain interior. While $\rho^{\mathrm{GND}}$ goes to zero due to $\Theta, \bar{\tau}^{\mathrm{CSS}}$ has a non-zero minimum value (in this case 7.55 MPa) due to $\rho^{\text {SSD }}$. The hardening profile on either side 
of the grain boundary in Figure 6.17 mimics a 1/distance type of decay, which reasonable considering dislocation stress fields also decay as $1 /$ distance.

The width of the hardened grain boundary region is the physical length scale that dominates the dependence of yield strength on grain size. Since the width of similar grain boundary hardened regions is independent of grain size, a polycrysal with a smaller grain size produces a larger fraction of hardened material. This observation illustrates the primary mechanism responsible for the grain size dependant response. As the grain size gets smaller, the relative amount of hardened material increases because the width of grain boundary zone of influence remains constant. Therefore, the smaller grain sizes have a higher volume fraction of hardened material resulting in a material with a higher yield strength.

\subsection{Grain Size Dependence of Yield Strength in Copper}

The experimental Hall-Petch results from Fernandes and Viera (2000) and Hansen (1983) were used to validate the simulation results of the non-local model. The tensile response of polycrystalline copper with grain sizes between $14 \mu \mathrm{m}$ and $244 \mu \mathrm{m}$ was simulated. The $0.2 \%$ offset yield strength from the simulations is compared with the experimental results from the literature data in Figures 6.18 and 6.19. Qualitatively, the simulated data in Figures 6.18 and 6.19 fit the experimental data reasonably well since the simulated data falls within the experimental scatter. The one exception was the $14 \mu \mathrm{m}$ data point in Figure 6.18. If a line were fit to the data from the 4 larger grain sizes, the yield strength at $14 \mu \mathrm{m}$ would appear to be unreasonably high. In any case, the model attempts to capture this rapid rise in yield strength at $14 \mu \mathrm{m}$. 


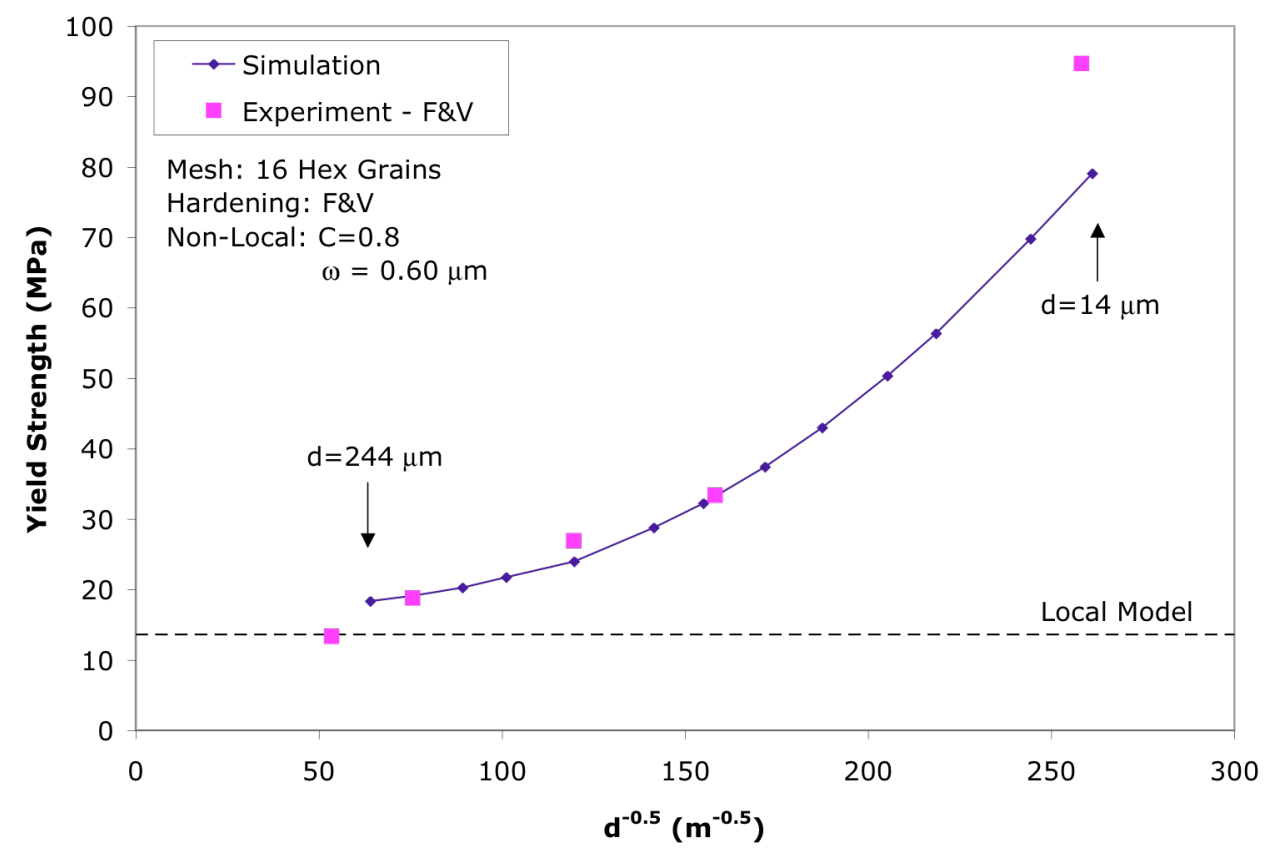

Figure 6.18 - Hall-Petch plot comparing experimental results from Fernandes and Viera (2000) to simulation results. The local model result is shown as a dashed line sine this result is grain size independent.

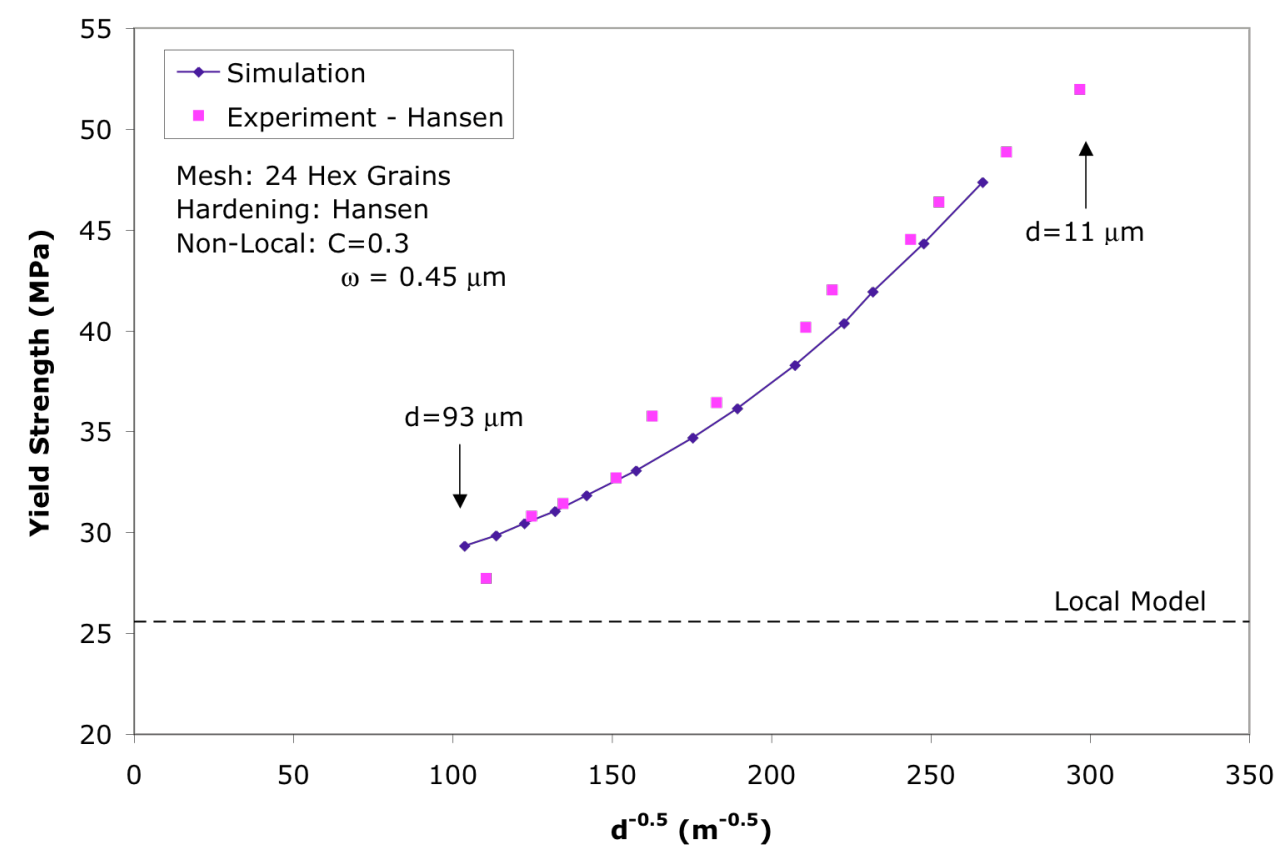

Figure 6.19 - Hall-Petch plot comparing experimental results from Hansen (1983) with simulation results. The local model result is shown as a dashed line since this result is grain size independent. 
The qualitative comparison between the best-fit Hall-Petch equations for the simulation and experimental results do not look quite as nice. These equations are presented in Table 6.4 along with an equation that represents an average experimental result. This average result was included in Table 6.4 to provide perspective on what type of values for Hall-Petch exponent (n) and the Hall-Petch slope (k) are considered reasonable for copper.

Table 6.4 -Best-fit Hall-Petch equations to simulation and experiment data

\begin{tabular}{|c|c|c|}
\hline & $\begin{array}{c}\text { Simulation } \\
\left(\sigma_{\mathrm{y}}=\sigma_{0}+\mathrm{k} \mathrm{d}^{-\mathrm{n}}\right)\end{array}$ & $\begin{array}{c}\text { Experiment } \\
\left(\sigma_{\mathrm{y}}=\sigma_{0}+\mathrm{k} \mathrm{d}^{-\mathrm{n}}\right)\end{array}$ \\
\hline Fernandes and Viera & $\sigma_{\mathrm{y}}=16.47+0.0000288 \mathrm{~d}^{-1.31}$ & $\sigma_{\mathrm{y}}=14.96+0.00000692 \mathrm{~d}^{-1.47}$ \\
\hline Hansen & $\sigma_{\mathrm{y}}=27.15+0.0000391 \mathrm{~d}^{-1.18}$ & $\sigma_{\mathrm{y}}=14.216+0.126 \mathrm{~d}^{-0.50}$ \\
\hline $\begin{array}{c}\text { Experimental } \\
\text { Average* }\end{array}$ & -------- & $\sigma_{\mathrm{y}}=16.09+0.143 \mathrm{~d}^{-0.50}$ \\
\hline
\end{tabular}

* The experimental average equation is an average of 5 experimental results on copper. See Appendix C for details.

The equations in Table 6.4 illustrate a number of important points:

- The value of $\mathrm{n}$ for both of the simulation curves is much higher than the traditional -0.5 indicating that the simulation results have some curvature in $\mathrm{d}^{-0.5}$ space. This curvature can be clearly seen in Figures 6.18 and 6.19.

- The value of $\mathrm{k}$ for both simulation curves is much lower (by about 4 orders of magnitude) than the values for Hansen experiment or the average experimental result. Unfortunately, it is not possible to compare $\mathrm{k}$ values by themselves for equations with such different $\mathrm{n}$ values because $\mathrm{k}$ and $\mathrm{n}$ are highly correlated parameters that should always be used as a set. 
- The large exponent in the Fernandes and Viera simulations is due to the high yield strength at $\mathrm{d}=14 \mu \mathrm{m}$. The model attempts to capture this rapid rise in yield strength and consequently the simulation results also have a large Hall-Petch exponent.

Since comparing k values for curves with different n's is not possible, the rest of the discussion focuses on $\mathrm{n}$.

The $\mathrm{n}$ values from the simulations are closer to $\mathrm{d}^{-1}$ than $\mathrm{d}^{-0.5}$ suggesting that the non-local model is no better than Kocks's composite model (Kocks, 1970). See Appendix B for details on Kocks's analysis. Any approach in which different regions of a material have different properties (e.g. grain boundaries that are "harder" than the interiors) is essentially a composite model. However, there are some fundamental differences between Kocks's composite model and the non-local model. The non-local model produces a graded, not step-function like, hardening profile near grain boundaries. The width of the grain boundary hardened region depends on the nature of the boundary itself (i.e. the misorientation between the two grains) and therefore does not need to be predefined. Finally, the width of the grain boundary hardened region can evolve with deformation if the grain boundary character changes. These differences illustrate the ways in which the non-local model is more physical and therefore superior to Kocks's composite model.

It bears mentioning that another possible cause for the $\mathrm{d}^{-1}$ dependence is the 2D nature of the simulation. The PBC used in the z-direction give the grains a columnar shape in 3D. This grain structure is clearly not physical, but computational limitations as 
well as the lack of experimental data about the grain structure in the $3^{\text {rd }}$ dimension restrict the simulations to their current form.

\subsection{The Effect of Simulation Parameters on the Hall-Petch Response}

The non-local model predicts a $\mathrm{d}^{-1}$ like dependence on yield strength when the best-fit parameters were applied to a polycrystal made up of 24 hexagon shaped grains. Without investigating the influence of various simulation parameters, it was not clear whether or not the $\mathrm{d}^{-1}$ dependence was inherent to the model or caused by one or more of the simulation parameters. Therefore a number of studies were conducted on the following parameters:

- Grain Shape

- $\quad \mathrm{C}$ and $\omega$

- Mesh refinement

- Form of $\Theta$ function

- Misorientation distribution

A sub-section is devoted to each of these 5 parameters.

\subsubsection{Effect of Grain Shape on the Hall-Petch Response}

The results in Section 6.3 were based on simulations of polycrystals containing hexagonal shaped grains. Polycrystals with hexagonal shaped grains are a useful computation tool because they are easy to construct and mesh. However, a polycrystal 
with hexagon shaped grains is not a physical reality. Therefore the effect of grain shape on the Hall-Petch response was investigated.

In this study, the grain size dependence of yield strength for polycrystalline copper was simulated using three different grain shapes: 1) 24 square grains, 2) 24 hexagonal grains, and 3) 25 realistic grains. In order to make a fair comparison among these three different polycrystals' Hall-Petch response, an "average" orientation (Section 6.1.1) for each of the polycrystals was determined. The large grain size yield strength predicted by the local model for each average orientation was similar meaning the baseline yield strength for each grain shape was also similar. While the orientations for each polycrystal were not the same, the same elastic, hardening, and non-local parameters were used in all the simulations.

A Hall-Petch plot containing the square grain, hexagon grain, and realistic grain polycrystal results is shown in Figure 6.20, and the best fit Hall-Petch equation for each polycrystal is in Table 6.5 . 


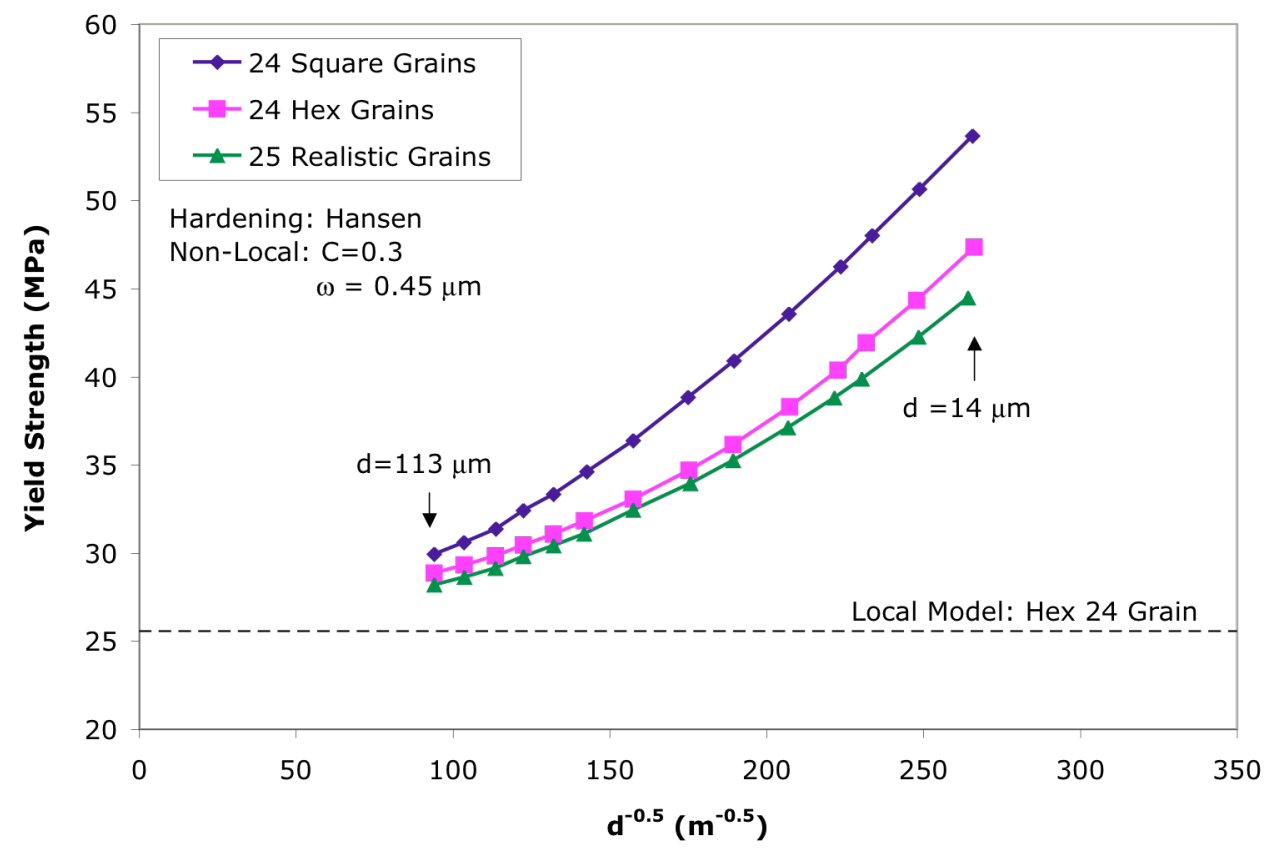

Figure 6.20 - The simulated Hall-Petch response for a polycrystal made up of square, hexagon and realistic shaped grains.

The simulated Hall-Petch results show the grain shape effect is greatest for small grains, and as grain size gets larger all the curves converge to values between $26 \mathrm{MPa}$ and $27 \mathrm{MPa}\left(\sigma_{0}\right.$ value in Table 6.5). This convergence was expected since all three grain shapes had similar large grain size yield strengths. Figure 6.20 also shows that square grains produce a higher yield strength for a given grain size than hex and realistic grains. The reason for this trend is that square grains have more hardened grain boundary material aligned along the loading axis. The hardened grain boundary material aligned with the loading axis supports more load making the polycrystal stronger, much like fibers in composite materials.

The Hall-Petch equations in Table 6.5 reveal that the polycrystal with square shaped grains produced the lowest Hall-Petch exponent, followed by polycrystals made up of realistic and hex shaped grains. 
Table 6.5 - Best-fit Hall-Petch equations for a polycrystal composed of square, hexagonal, and realistic shape grains.

\begin{tabular}{|l|c|c|}
\hline & $\begin{array}{c}\sigma_{\mathrm{ys}}(\mathrm{MPa}) \\
\text { Large Grain }\end{array}$ & $\begin{array}{c}\text { Hall-Petch Equation } \\
\left(\sigma_{\mathrm{y}}=\sigma_{0}+\mathrm{k} \mathrm{d}^{-\mathrm{n}}\right)\end{array}$ \\
\hline 24 Square Grains & 25.53 & $\sigma_{\mathrm{y}}=25.62+0.00104 \mathrm{~d}^{-0.91}$ \\
\hline 24 Hex Grains & 25.58 & $\sigma_{\mathrm{y}}=27.15+0.0000391 \mathrm{~d}^{-1.18}$ \\
\hline 25 Realistic Grains & 26.09 & $\sigma_{\mathrm{y}}=25.98+0.000189 \mathrm{~d}^{-1.03}$ \\
\hline Experimental Average* & ----- & $\sigma_{\mathrm{y}}=16.09+0.143 \mathrm{~d}^{-0.50}$ \\
\hline
\end{tabular}

* See Appendix $\mathrm{C}$ for details.

It is interesting to note that even though the yield strength of a polycrystal with square shaped grains is higher than the other grain shapes, the curvature in the Hall-Petch response is lower than the other grain shapes. The important message in Figure 6.20 and Table 6.5 is that grain shape does affect the Hall-Petch response, but grain shape is not solely responsible for the $\mathrm{d}^{-1}$ dependence.

\subsubsection{Effect of $C$ and $\omega$ on the Hall-Petch Response}

In Section 6.1.4, it was stated that the Hall-Petch response in the simulations was fit by adjusting $\mathrm{C}$ and $\omega$. However, no explanation was given in Section 6.1.4 about how these two parameters affect the dependence of yield strength on grain size. These details are covered in this section.

The $\mathrm{C}$ parameter in the Taylor equation $\left(\tau^{\mathrm{CSS}}=\mathrm{CGb} \sqrt{\langle\rho\rangle}\right)$ acts as a damping term on the Hall-Petch response: a higher value of $\mathrm{C}$ results in a higher yield strength for a given grain size. To illustrate this point, a number of simulations were conducted with $\mathrm{C}$ 
values ranging from 0.2-0.8. The results of these simulations are shown in Figure 6.21 and the best fit Hall-Petch equations are in Table 6.6.

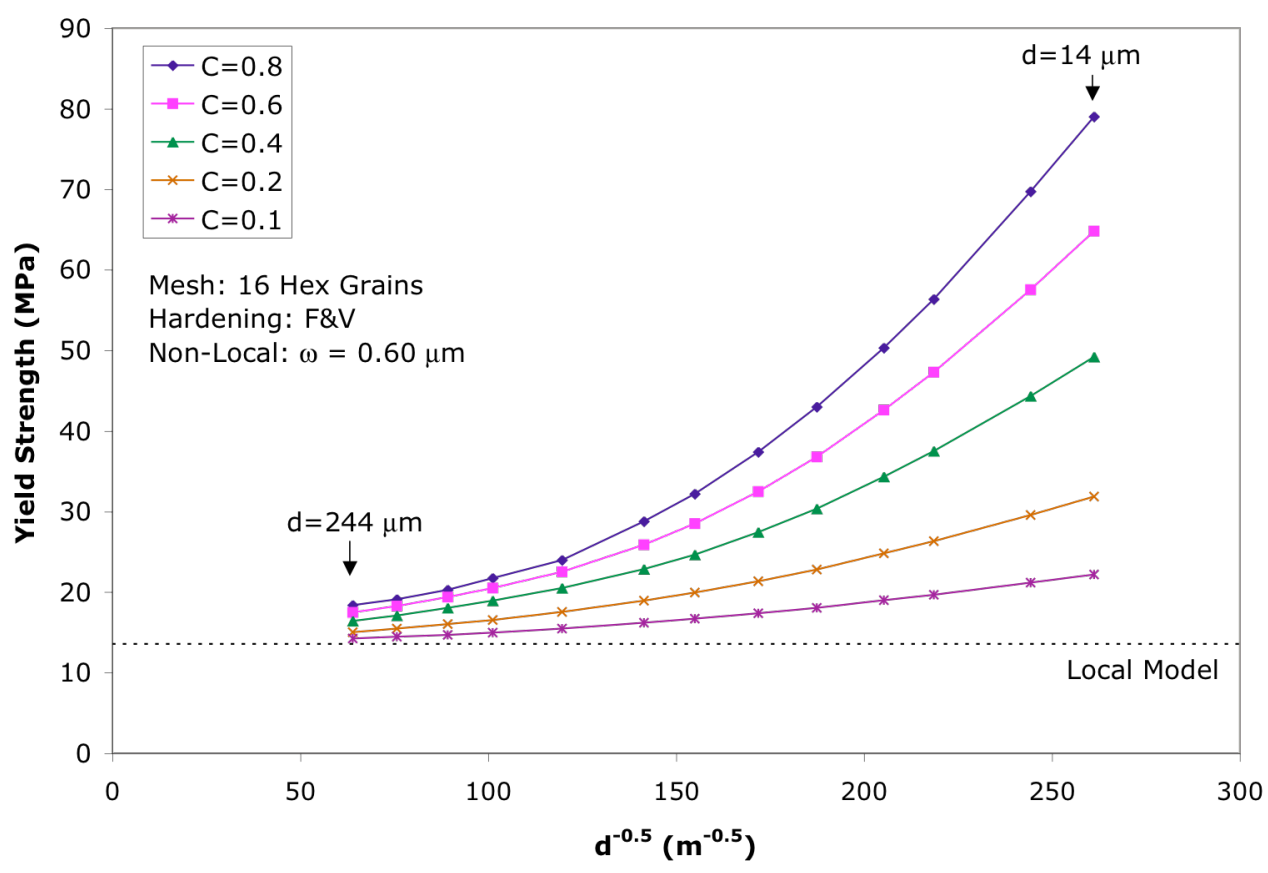

Figure 6.21 - The dependence of the simulated Hall-Petch response on C.

Table 6.6 - Best-fit Hall-Petch equations for simulations with varying $\mathrm{C}$ values.

\begin{tabular}{|c|c|c|}
\hline$\omega(\mu \mathrm{m})$ & $\mathrm{C}$ & $\begin{array}{c}\text { Hall-Petch Equation } \\
\left(\sigma_{\mathrm{y}}=\sigma_{0}+\mathrm{k} \mathrm{d}^{-\mathrm{n}}\right)\end{array}$ \\
\hline 0.60 & 0.8 & $\sigma_{\mathrm{y}}=16.47+0.0000288 \mathrm{~d}^{-1.31}$ \\
\hline 0.60 & 0.6 & $\sigma_{\mathrm{y}}=16.28+0.0000238 \mathrm{~d}^{-1.31}$ \\
\hline 0.60 & 0.4 & $\sigma_{\mathrm{y}}=15.71+0.0000350 \mathrm{~d}^{-1.24}$ \\
\hline 0.60 & 0.2 & $\sigma_{\mathrm{y}}=14.25+0.000111 \mathrm{~d}^{-1.08}$ \\
\hline 0.60 & 0.1 & $\sigma_{\mathrm{y}}=13.72+0.000126 \mathrm{~d}^{-1.00}$ \\
\hline
\end{tabular}

Adjusting the C parameter affects both the curvature (n) and the slope (k) of the Hall-

Petch response. As $\mathrm{C}$ gets larger, both $\mathrm{n}$ and $\mathrm{k}$ decrease. As $\mathrm{C} \rightarrow 0, \mathrm{n}$ gets smaller and $\mathrm{k}$ 
gets larger, but the change in $\sigma_{\mathrm{ys}}$ between $\mathrm{d}=14 \mu \mathrm{m}$ and $\mathrm{d}=244 \mu \mathrm{m}$ becomes smaller. A C value very close to zero will render the model grain size insensitive, much like the local model.

The $\omega$ parameter also affects the yield strength - grain size dependence. In the non-local model, the $\omega$ parameter determines how fast the $\Theta$ function decays to zero. As $\omega$ gets larger, $\Theta$ decays slower and has a lower maximum value resulting in a larger but less intensely hardened grain boundary region. As the grain boundary region gets larger and less intense, the $\sigma_{\mathrm{ys}}$ for a given grain size will be higher, as seen in Figure 6.22.

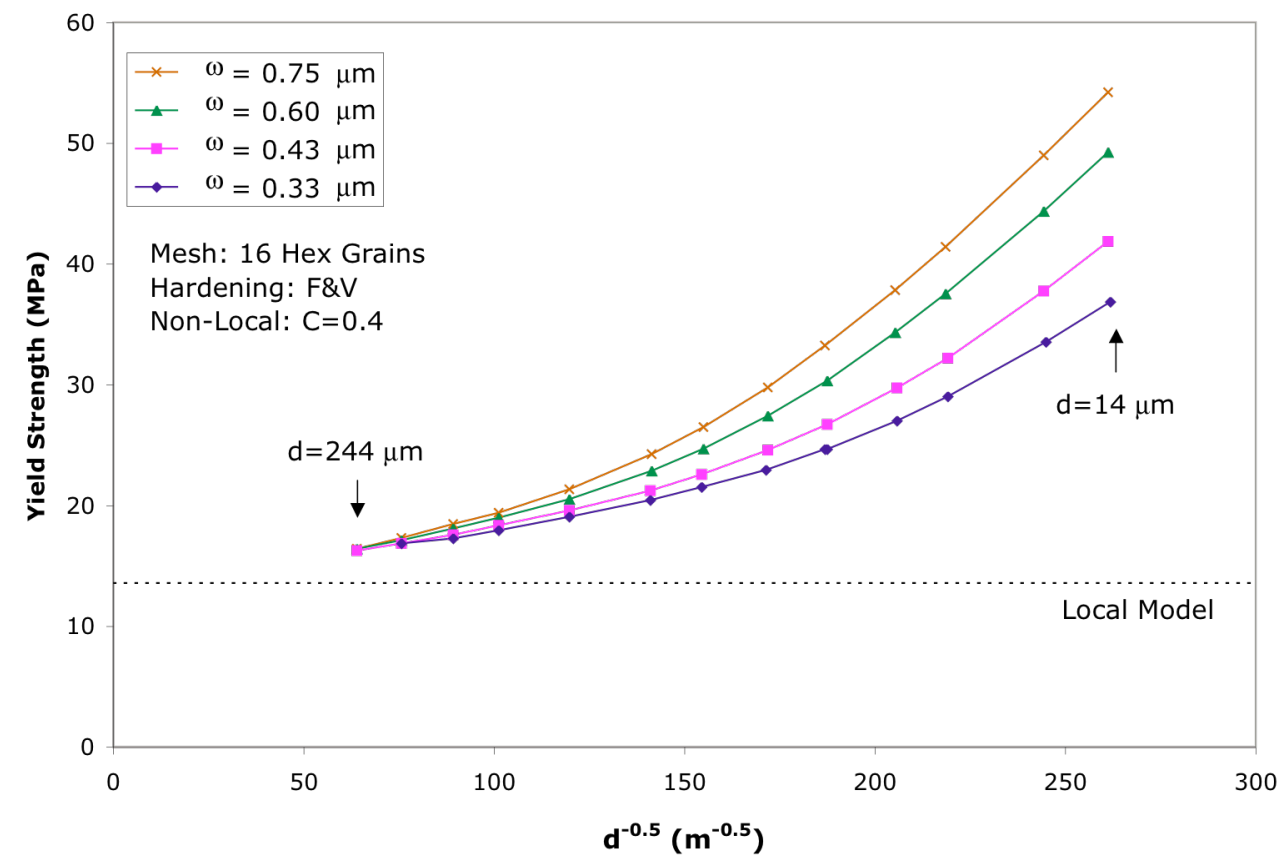

Figure 6.22 - The dependence of the simulated Hall-Petch response on $\omega$ 
Table 6.7 - Best-fit Hall-Petch equations for simulations with varying $\omega$ values.

\begin{tabular}{|c|c|c|}
\hline$\omega(\mu \mathrm{m})$ & $\mathrm{C}$ & $\begin{array}{c}\text { Hall-Petch Equation } \\
\left(\sigma_{\mathrm{y}}=\sigma_{0}+\mathrm{k} \mathrm{d}^{-\mathrm{n}}\right)\end{array}$ \\
\hline 0.75 & 0.4 & $\sigma_{\mathrm{y}}=14.98+0.0000964 \mathrm{~d}^{-1.16}$ \\
\hline 0.60 & 0.4 & $\sigma_{\mathrm{y}}=15.71+0.0000350 \mathrm{~d}^{-1.24}$ \\
\hline 0.43 & 0.4 & $\sigma_{\mathrm{y}}=15.90+0.0000141 \mathrm{~d}^{-1.30}$ \\
\hline 0.33 & 0.4 & $\sigma_{\mathrm{y}}=16.23+0.00000615 \mathrm{~d}^{-1.32}$ \\
\hline
\end{tabular}

Much like the $\mathrm{C}$ parameter, $\omega$ affects both the curvature (n) and the slope (k) of the HallPetch response. As $\omega$ gets larger (resulting in a larger hardened grain boundary region), $n$ decreases and $\mathrm{k}$ increases. These $\omega$ results are interesting because they suggest that a larger but less intense grain boundary region will provide a Hall-Petch response that is closer to observed experimental trends. This idea is investigated further in Section 6.6.

\subsubsection{Effect of Mesh Refinement on the Hall-Petch Response}

Mesh resolution is an important parameter to the non-local integral based gradient approximation. If the mesh is too coarse for a given $\omega$, then the non-local integral technique breaks down and converges to a non-zero value in the closet element (Section 5.3). To illustrate how this error affects the Hall-Petch response, a mesh containing 8000 elements was used to simulate the yield strength of polycrystals with a grain size between $1 \mathrm{~cm}$ and $15 \mu \mathrm{m}$ with a $\omega$ of $0.74 \mu \mathrm{m}$. A Hall-Petch plot containing the results from the 8000 element mesh as well as a converged result is shown in Figure 6.23. 


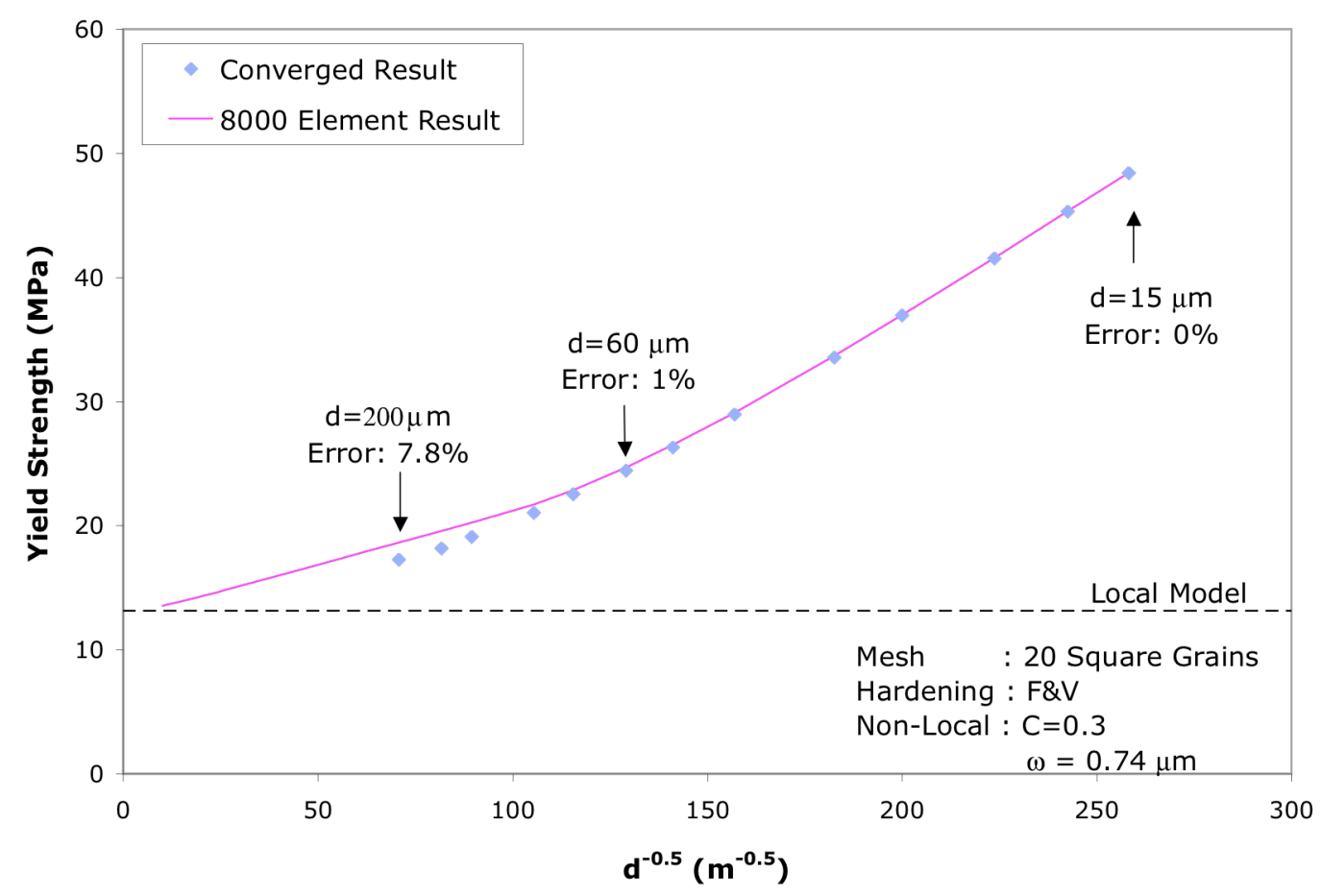

Figure 6.23 - The effect of mesh refinement on the Hall-Petch response

At small grain sizes, the mesh resolution is appropriate for $\omega$ and the 8000 element mesh result is the converged result. At $\mathrm{d}=60 \mu \mathrm{m}$ the difference in $\sigma_{\mathrm{y}}$ between the 8000 element mesh and a more refined mesh (12000 elements) is more than $1 \%$. This error is the result of the mesh being too coarse for the given $\omega$. Therefore the converged result uses meshes more refined than 8000 elements for grain sizes larger than $60 \mu \mathrm{m}$. The error in the 8000 element result increases as grain size gets larger and at $200 \mu \mathrm{m}$ the error is $7.8 \%$. The effect of this error on the Hall-Petch response is quantified in Table 6.8, which contains the best fit Hall-Petch equations. 
Table 6.8 - Best-fit Hall-Petch Equations for converged and non-converged simulations.

\begin{tabular}{|c|c|c|}
\hline & Data Range & $\begin{array}{c}\text { Hall-Petch Equation } \\
\left(\sigma_{\mathrm{y}}=\sigma_{0}+\mathrm{k} \mathrm{d}^{-\mathrm{n}}\right)\end{array}$ \\
\hline Converged Result & $15 \mu \mathrm{m}-200 \mu \mathrm{m}$ & $\sigma_{\mathrm{y}}=12.06+0.00681 \mathrm{~d}^{-0.77}$ \\
\hline 8000 Element Result & $15 \mu \mathrm{m}-200 \mu \mathrm{m}$ & $\sigma_{\mathrm{y}}=14.75+0.00225 \mathrm{~d}^{-0.87}$ \\
\hline 8000 Element Result & $15 \mu \mathrm{m}-1 \mathrm{~cm}$ & $\sigma_{\mathrm{y}}=13.94+0.00431 \mathrm{~d}^{-0.81}$ \\
\hline Experimental Average* & ------- & $\sigma_{\mathrm{y}}=16.09+0.143 \mathrm{~d}^{-0.50}$ \\
\hline
\end{tabular}

*See Appendix C for details.

All yield strength results presented in this disseration are converged. These results confirm the need to check the convergence of the yield strength results. The converged result has a higher slope $(\mathrm{k})$ and lower curvature $(\mathrm{n})$ compared to the non-converged result. Both of these trends bring the Hall-Petch result in better agreement with experiments.

\subsubsection{Effect of the $\Theta$ Function on the Hall-Petch Response}

One common factor in all the simulations presented thus far is the use of $\Theta=\operatorname{Exp}$ as the attenuation function in the non-local integrals. At this point, it was not clear whether the $\mathrm{d}^{-1}$ dependence was caused by the exponential attenuation function. Therefore, a Gaussian attenuation function was used in the non-local integrals in order to investigate the effect of the $\Theta$ function on the Hall-Petch response. 
Non-local simulations with the Gaussian $\Theta$ function and different $\omega$ values were completed. The results of these simulations are compared in Figure 6.24 to a simulation data set generated with a $\omega=0.45 \mu \mathrm{m}$ in exponential $\Theta$ function.

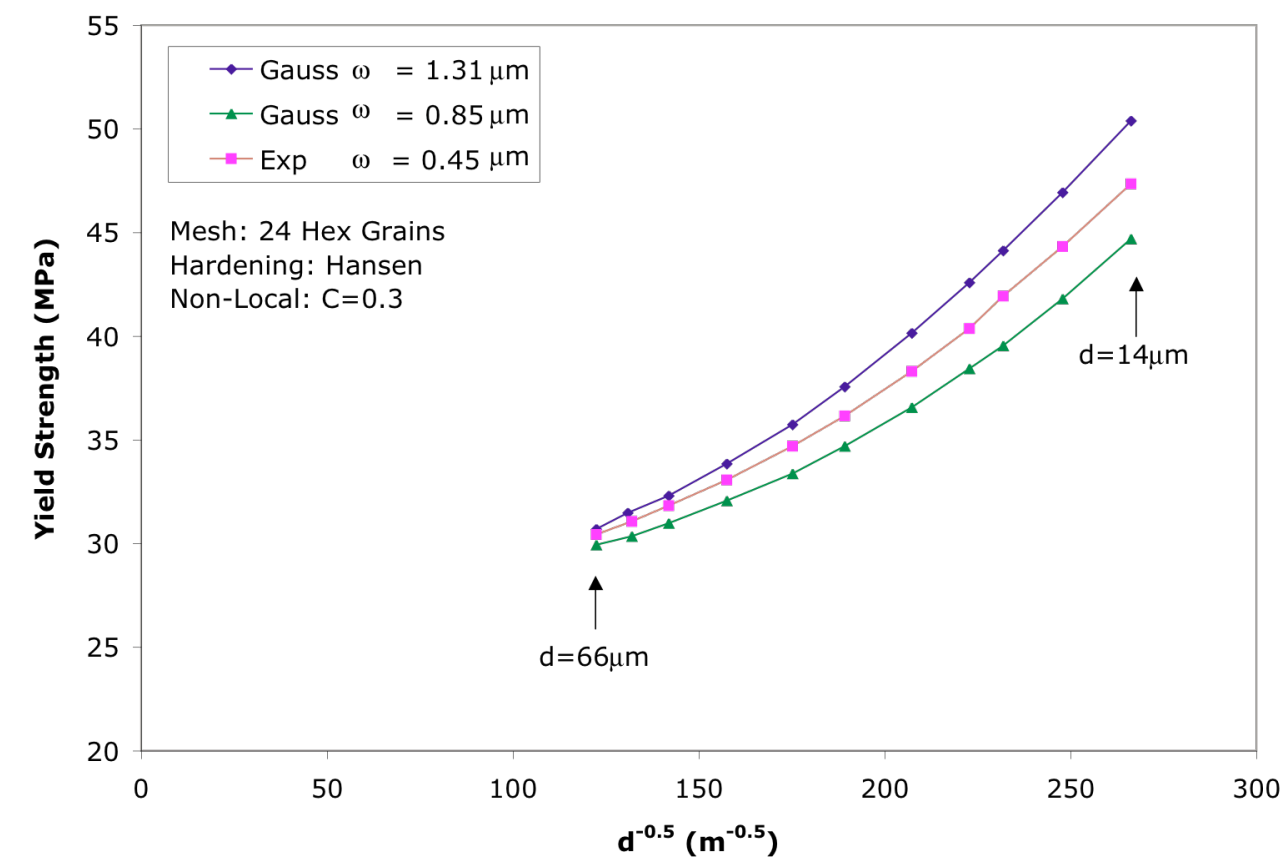

Figure 6.24 - Simulated Hall-Petch curves from a non-local model using a Gaussian $\Theta$ function.

The difference in the Hall-Petch response between a non-local model based on a Gaussian $\Theta$ function and one based on an exponential $\Theta$ function is the magnitude of $\omega$ needed for a given response. The results in Figure 6.24 suggest that a Gaussian $\omega$ (an $\omega$ used in a Gaussian function) between $0.85 \mu \mathrm{m}-1.31 \mu \mathrm{m}$ will result in exactly the same response as that for an exponential $\omega$ equal to $0.45 \mu \mathrm{m}$. The reason an exponential $\omega$ will be greater than a Gaussian $\omega$ for a given Hall-Petch response has to do with the rate at which the Gauss and exponential functions decay. For the same $\omega$, the Gauss function 
decays faster than the exponential function. Therefore, an exponential $\omega$ must be larger than a Gaussian $\omega$ for the two functions to decay at the same rate.

The Hall-Petch equations in Table 6.9 confirm the general similarities between the Gaussian and exponential Hall-Petch responses.

Table 6.9 - Best-fit Hall-Petch equations to simulation results using a Gaussian $\Theta$ function.

\begin{tabular}{|c|c|c|}
\hline & Data Range & $\begin{array}{c}\text { Hall-Petch Equation } \\
\left(\sigma_{\mathrm{y}}=\sigma_{0}+\mathrm{k} \mathrm{d}^{-\mathrm{n}}\right)\end{array}$ \\
\hline Gaussian; $\omega=1.31 \mu \mathrm{m}$ & $14 \mu \mathrm{m}-66 \mu \mathrm{m}$ & $\sigma_{\mathrm{y}}=26.46+0.0000849 \mathrm{~d}^{-1.12}$ \\
\hline Gaussian; $\omega=0.85 \mu \mathrm{m}$ & $14 \mu \mathrm{m}-66 \mu \mathrm{m}$ & $\sigma_{\mathrm{y}}=27.76+0.00000722 \mathrm{~d}^{-1.31}$ \\
\hline Exponential; $\omega=0.45 \mu \mathrm{m}$ & $14 \mu \mathrm{m}-66 \mu \mathrm{m}$ & $\sigma_{\mathrm{y}}=27.32+0.0000313 \mathrm{~d}^{-1.20}$ \\
\hline Experimental Average* & ------- & $\sigma_{\mathrm{y}}=16.09+0.143 \mathrm{~d}^{-0.50}$ \\
\hline
\end{tabular}

*See Appendix C for details.

The Hall-Petch behavior predicted by both $\Theta$ functions has a $\mathrm{d}^{-1}$ like dependence. In addition, as the Gaussian $\omega$ increases, $\mathrm{k}$ decreases and $\mathrm{n}$ increases, which is exactly the same trends observed for exponential $\omega$ 's (See Section 6.4.2). The results in Figure 6.24 and Table 6.9 prove that the $\mathrm{d}^{-1}$ dependence predicted by the non-local model is not the result of the exponential $\Theta$ function.

\subsubsection{Effect of the Misorientation Distribution on the Hall-Petch Response}

The polycrystals used in the Hall-Petch simulations contained between 16-25 grains. Because 16-25 grains constitutes a small statistical population, an "average" orientation (Section 6.1.1) was determined for each polycrystal to ensure that the small 
orientation set did not have a strong effect on the stress-strain response. However, this "average" orientation was based on the macroscopic response of the material and does not guarantee that the misorientation distribution within the polycrystal is realistic. The initial misorientation distribution associated with the orientation field is particularly important in the calculation of $\{\tilde{\mathbf{G}}\}$. If the initial misorientation distribution is skewed toward high angle boundaries, the effect of $\rho_{\mathrm{t}=0}^{\mathrm{GND}}$ will be overstated. Conversely, if the initial misorientation distribution is skewed towards low angle boundaries, the effect of $\rho_{\mathrm{t}=0}^{\mathrm{GND}}$ will be understated. To gain some understanding into the effect of the misorientation distribution on the Hall-Petch response, the grain size effect of 3 polycrystals, each with a different misorientation distribution, was simulated.

In order to make a valid comparison, 3 different orientation sets with a large grain size $\sigma_{\mathrm{y}}$ that was close to the "average" $\sigma_{\mathrm{y}}$ were generated. The misorientation distribution for these three orientation sets were compared against the MacKenzie distribution (MacKenzie, 1958), which describes the misorientation distribution in a polycrystal containing a large number of randomly oriented grains. The comparison between the MacKenzie distribution and misorientation distribution for of the 3 generated orientation sets is shown in Figure 6.25. 


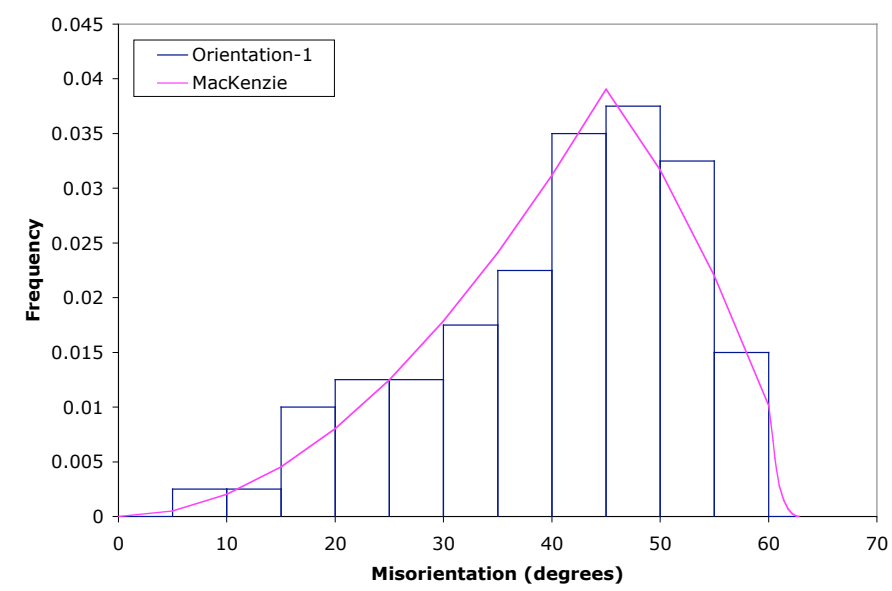

(a) Orientation-1

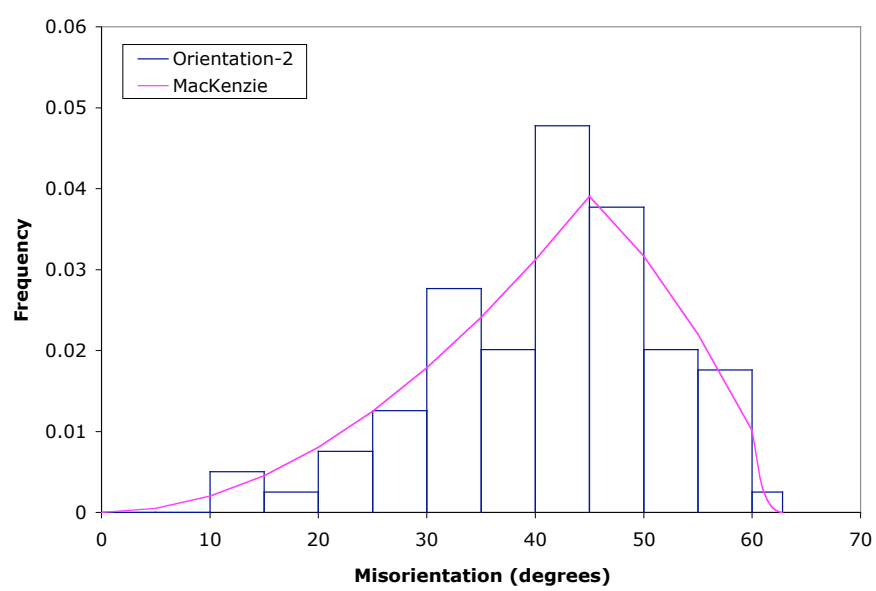

(b) Orientation-2

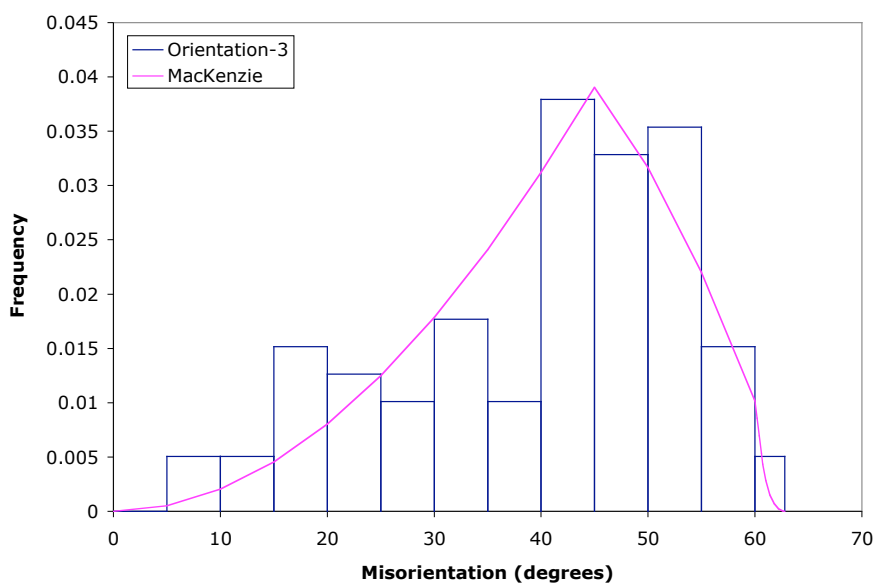

(c) Orientation-3

Figure 6.25 - Comparison of misorientation distributions with the MacKenzie distribution. 
The $1^{\text {st }}$ orientation, Orientation- 1 , had a misorientation distribution close to the MacKenzie distribution. Orientation-2 had a concentration of misorientations between $40^{\circ}-45^{\circ}$, and Orientation-3 had a bimodal like misorientation distribution with a disproportionate number of low and high angle boundaries.

The simulated Hall-Petch response for the 3 polycrystals is shown in Figure 6.26 and the best-fit Hall-Petch equations for each orientation set are contained in Table 6.10.

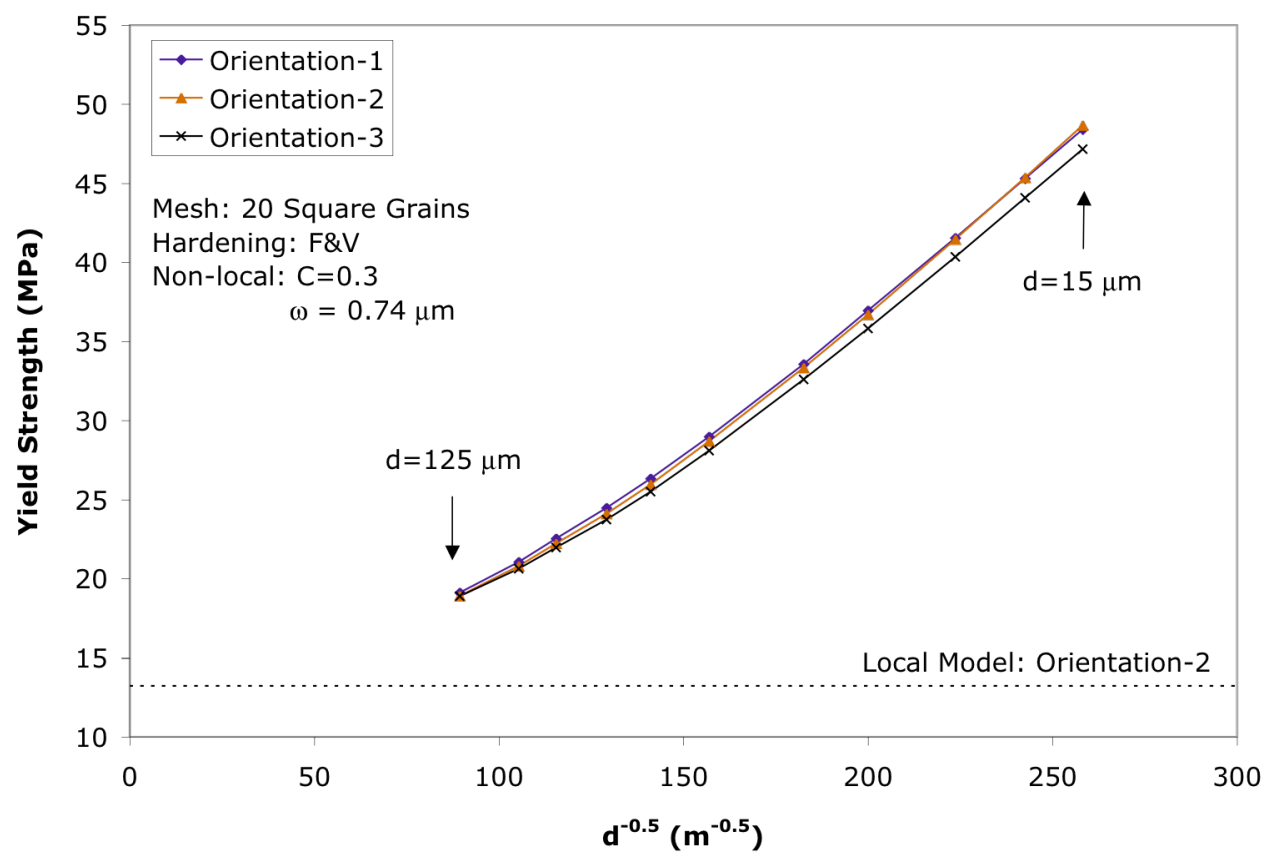

Figure 6.26 - The effect of misorientation distribution on the Hall-Petch response.

Table 6.10 - Best-fit Hall-Petch equations for the 3 different misorientation distributions

\begin{tabular}{|l|c|c|}
\hline & $\begin{array}{c}\sigma_{\mathrm{ys}}(\mathrm{MPa}) \\
\text { Large Grain }\end{array}$ & $\begin{array}{c}\text { Hall-Petch Equation } \\
\left(\sigma_{\mathrm{y}}=\sigma_{0}+\mathrm{k} \mathrm{d}^{-\mathrm{n}}\right)\end{array}$ \\
\hline Orientation-1 & 13.11 & $\sigma_{\mathrm{y}}=11.22+0.00984 \mathrm{~d}^{-0.74}$ \\
\hline Orientation-2 & 13.21 & $\sigma_{\mathrm{y}}=11.73+0.00613 \mathrm{~d}^{-0.78}$ \\
\hline Orientation-3 & 13.55 & $\sigma_{\mathrm{y}}=12.03+0.00565 \mathrm{~d}^{-0.79}$ \\
\hline Experimental Average* & ------- & $\sigma_{\mathrm{y}}=16.09+0.143 \mathrm{~d}^{-0.50}$ \\
\hline
\end{tabular}

*See Appendix $\mathrm{C}$ for details. 
From the graphs and the best fit equations it is clear that the misorientation distribution does not have a strong affect on the Hall-Petch response. While the affect is not strong, the misorientation distribution that best fit the MacKenzie distribution (Orientation-1) had the lowest curvature (n) and the highest slope (k), both trends that are more in-line with experiments.

\subsection{The Effect of Boundary Conditions on the Non-Local Model's $\sigma-\varepsilon$ Response}

Up to this point, all the simulations were run using PBC. As stated in Section 6.1.6, PBC were used because the boundary conditions on $\left\{\operatorname{Curl} \mathbf{R}_{\mathbf{h}}^{\mathbf{e}^{-1}}\right\}$ and $\left\{\operatorname{Curl} \mathbf{F}^{\mathbf{P}}\right\}$ were ambiguous. Also in Section 6.1.6, two different boundary conditions, FSBC \#1 and

FSBC \#2, were proposed for $\left\{\operatorname{Curl} \mathbf{R}_{\mathbf{h}}^{\mathbf{e}^{-1}}\right\}$ and $\left\{\operatorname{Curl} \mathbf{F}^{\mathbf{P}}\right\}$. All three boundary conditions (FSBC \#1, FSBC \#2, and PBC) were implemented to determine the effect of different of the different free surface boundary conditions (FSBC \#1 vs. FSBC \#2) and the effect of displacement boundary conditions (PBC vs. FSBCs).

Non-local simulations were run using each of the three boundary conditions on the realistic 25 grain polycrystal out to $17 \%$ strain. The resulting stress-strain curves are shown in Figure 6.27. 


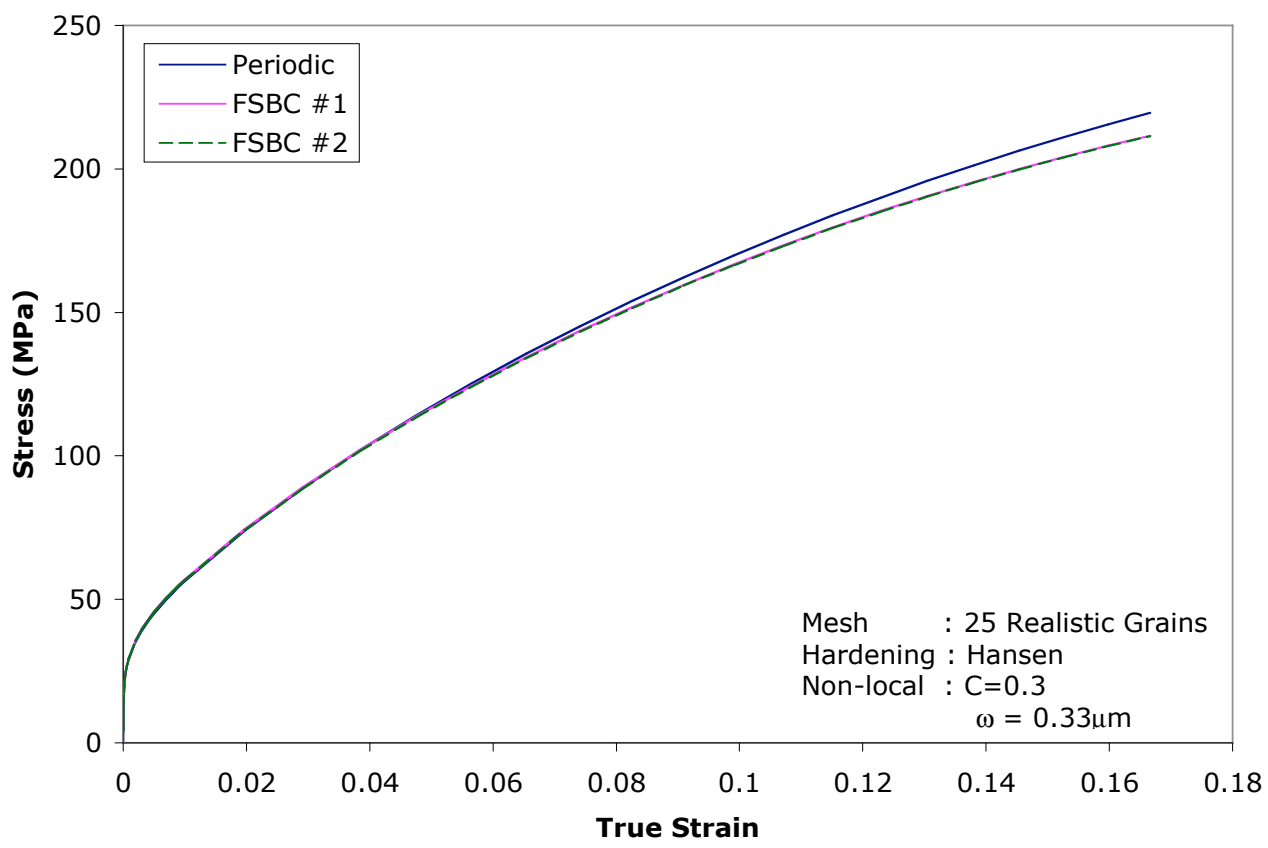

Figure 6.27 - A comparison of stress-strain curve generated with different boundary conditions.

The stress-strain curves in Figure 6.27 are basically identical up to 5\% strain, at which point the PBC curve becomes a little harder than the FSBC curves. At the yield point $(\varepsilon=0.2 \%)$, the three curves were all within $0.5 \mathrm{MPa}$, while at $17 \%$ strain the spread between the PBC curve and the FSBC curves had grown to $8 \mathrm{MPa}$ (a $3.5 \%$ difference) and the difference between FSBC \#1 and FSBC \#2 was only, 0.2 MPa. Therefore, the yield strength is essentially unaffected by the boundary conditions. Boundary conditions do noticeably affect the material response starting around 5\% strain, with PBC predicting a slightly harder $\sigma-\varepsilon$ response. However, the difference between the three boundary conditions is not great even at $17 \%$ strain.

The negligible difference between the two FSBCs was surprising. It was believed that the larger integration volume associated with FSBC \#2 would result in less grain boundary hardening at the specimen edges and therefore lead to a softer stress-strain 
response. However, an analysis of grain boundary hardening at the specimen edge revealed that FSBC \#2 did not significantly reduce boundary hardening at the specimen edges when compared with FSBC \#1. A hardening profile at $\mathrm{t}=0$ for a grain boundary that intersects the specimen edge is shown in Figure 6.28.

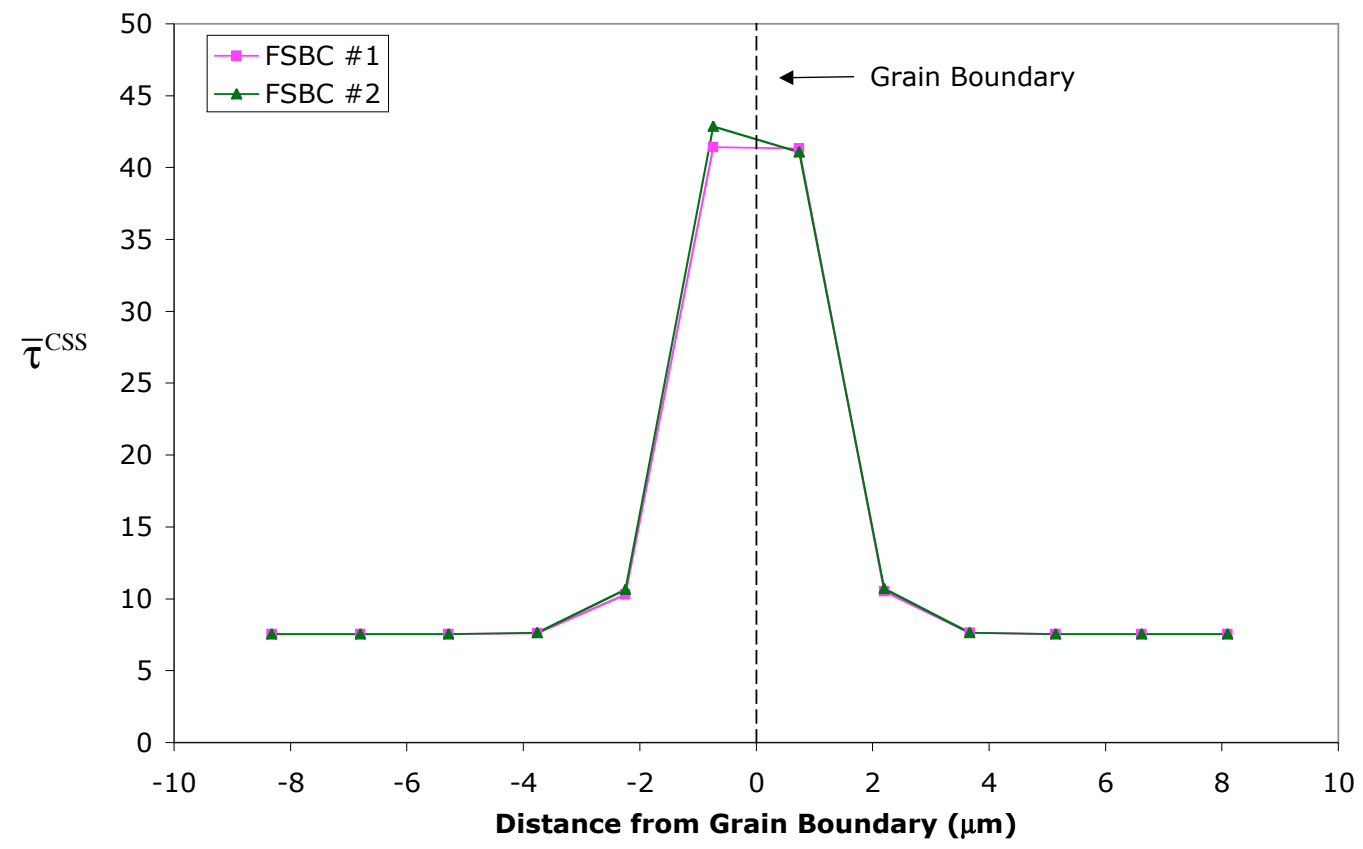

Figure 6.28 - A hardening profile generated by FSBC \#1 and FSBC \#2 for a grain boundary that intersects the specimen.

Since there is little difference between the initial material structures generated by FSBC $\# 1$ and FSBC \#2, it is not surprising that macroscopic stress-strain response was also indistinguishable. 


\subsection{Material Length Scale}

In the non-local model simulations, the hardened region around grain boundaries controlled the Hall-Petch response. With the purpose of investigating how grain boundaries affect the Hall-Petch response, two grain boundary properties were defined:

- Effective grain boundary layer width (GB layer width). Defined as the distance across the grain boundary between the two points where $\rho_{\mathrm{t}=0}^{\mathrm{SSD}}=\rho_{\mathrm{t}=0}^{\mathrm{GND}}$. GB layer width measures how far away from the grain boundary the GND hardening effects are felt.

- Effective grain boundary strength (GB strength). Defined as the difference between the maximum $\tau_{\mathrm{t}=0}^{\mathrm{CSS}}$ in the grain boundary zone and the $\tau_{\mathrm{t}=0}^{\mathrm{CSS}}$ in the grain interior. GB strength measures the strength of the GND hardening effects.

The definition of the GB layer width and GB strength is illustrated in Figure 6.29. 


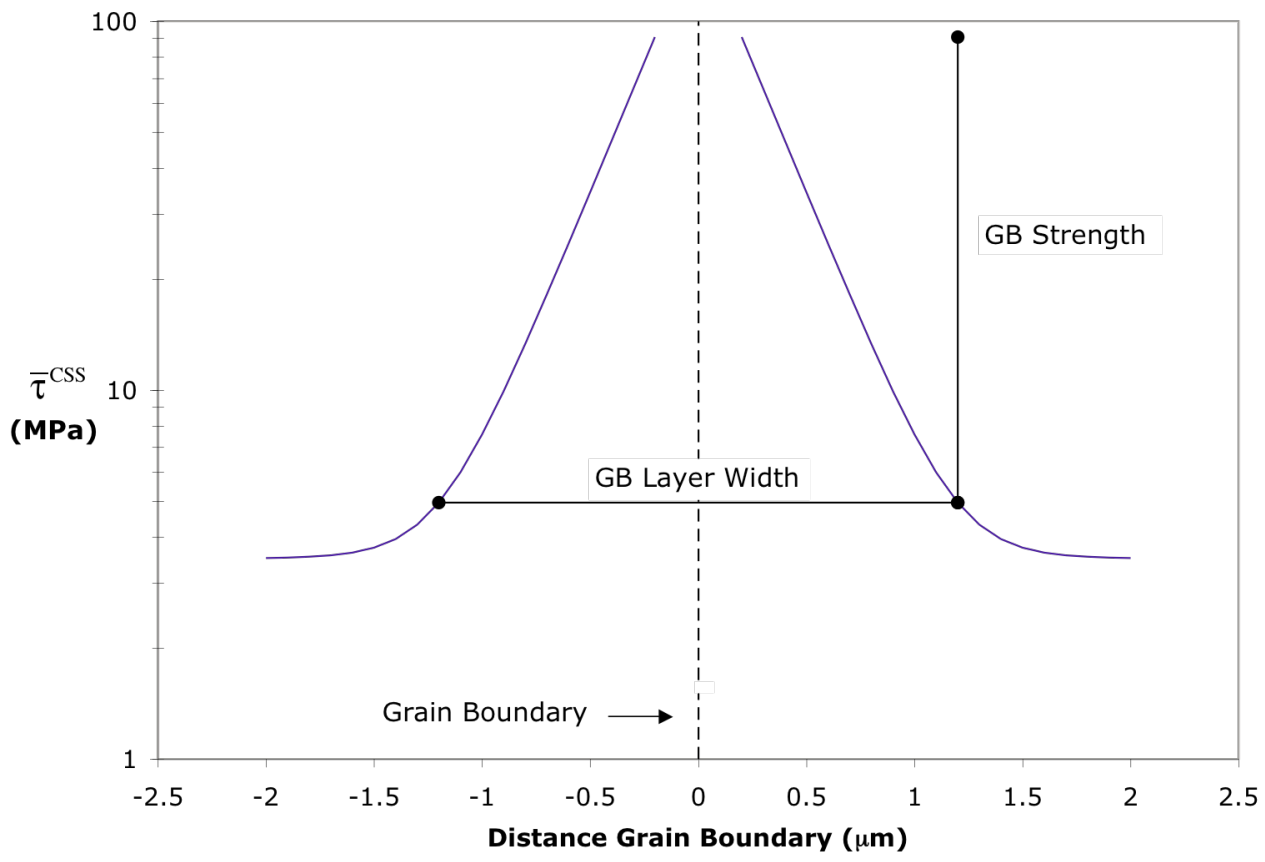

Figure 6.29 - The definition of GB layer width and GB strength.

The non-local model tracks all 12 FCC slip systems, so for each grain boundary there are 12 GB layer widths and 24 GB strengths (one on each side of the grain boundary). The material GB layer width was the average of all the slip system widths in the material, and the material GB strength was the average of all the slip system heights in the material.

The GB layer width and strength is controlled by the two non-local parameters C and $\omega$. Specifically, $\mathrm{C}$ affects the grain boundary width and height in two different ways. First decreasing $C$ increases $\rho_{t=0}^{\mathrm{SSD}}$, while leaving $\rho_{\mathrm{t}=0}^{\mathrm{GND}}$ unchanged, resulting in a smaller GB layer width and GB strength as shown in Figure 6.30. 


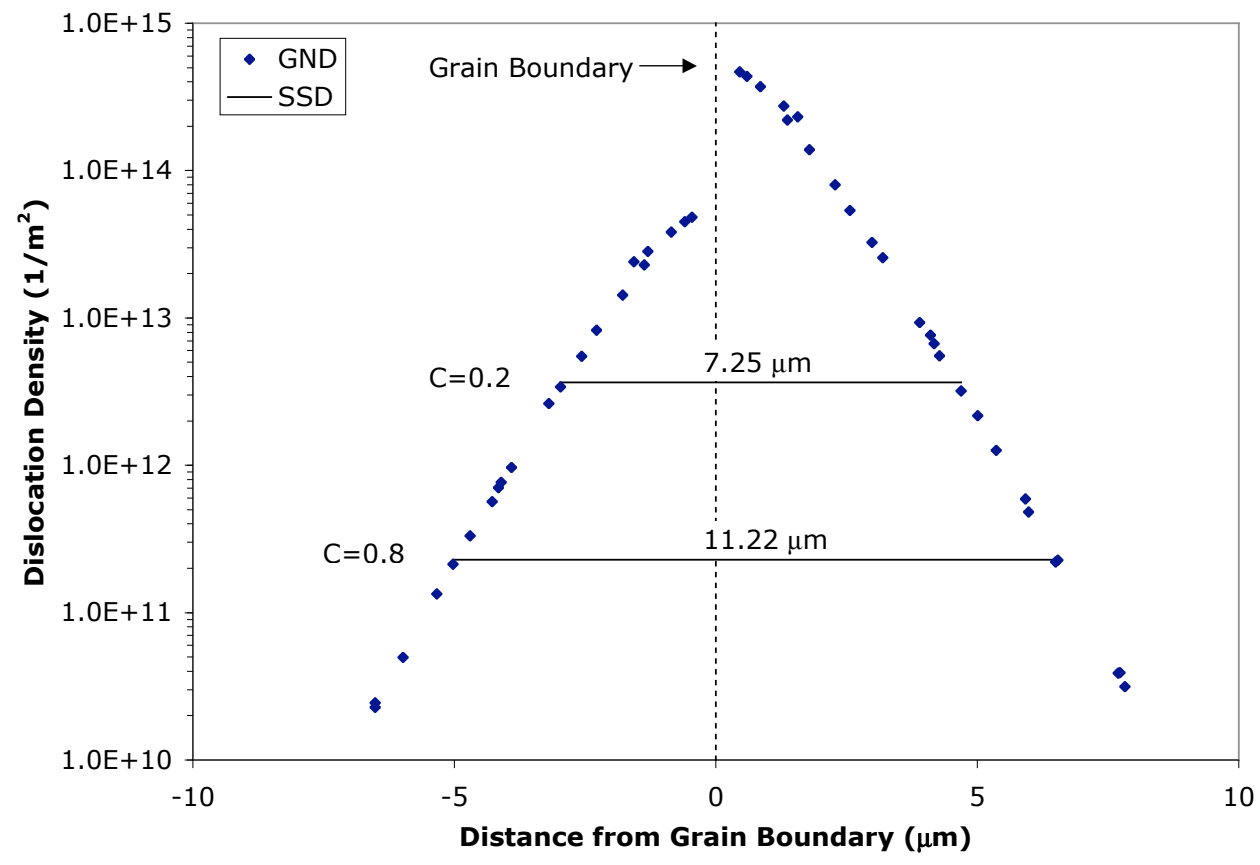

Figure 6.30 - The effect of changing $\rho_{\mathrm{t}=0}^{\mathrm{SSD}}$ with $\mathrm{C}$ on the material GB layer width

$\mathrm{C}$ also affects the overall hardening response as a damping like factor in the Taylor equation $\left(\tau^{\mathrm{CSS}}=\mathrm{CG}_{110} \mathrm{~b} \sqrt{\rho^{\mathrm{SSD}}+\rho^{\mathrm{GND}}}\right)$. Thus, decreasing $\mathrm{C}$ results in an additional decrease in the GB strength as shown in Figure 6.31. 


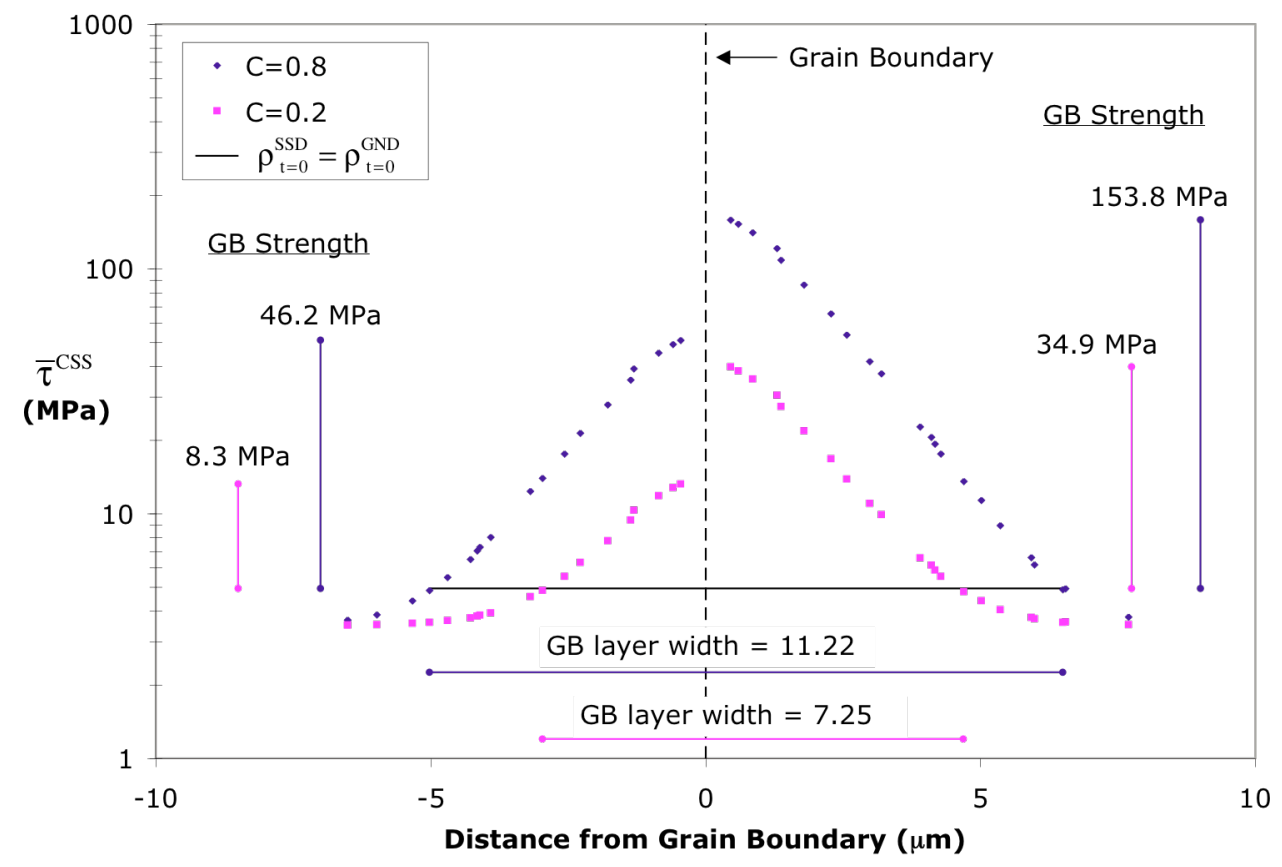

Figure 6.31 - The overall effect of C on the GB layer width and GB strength.

In general, decreasing $\mathrm{C}$ leads to a smaller GB layer width and a lower GB strength.

$\omega$, on the other hand, influences the material length scale through $\rho_{\mathrm{t}=0}^{\mathrm{GND}}$ only. As $\omega$ decreases, the exponential damping functions in the non-local integrals that describe $\rho^{\mathrm{GND}}$ decay faster. The effect of $\omega$ on grain boundary hardening is shown in Figure 6.32. 


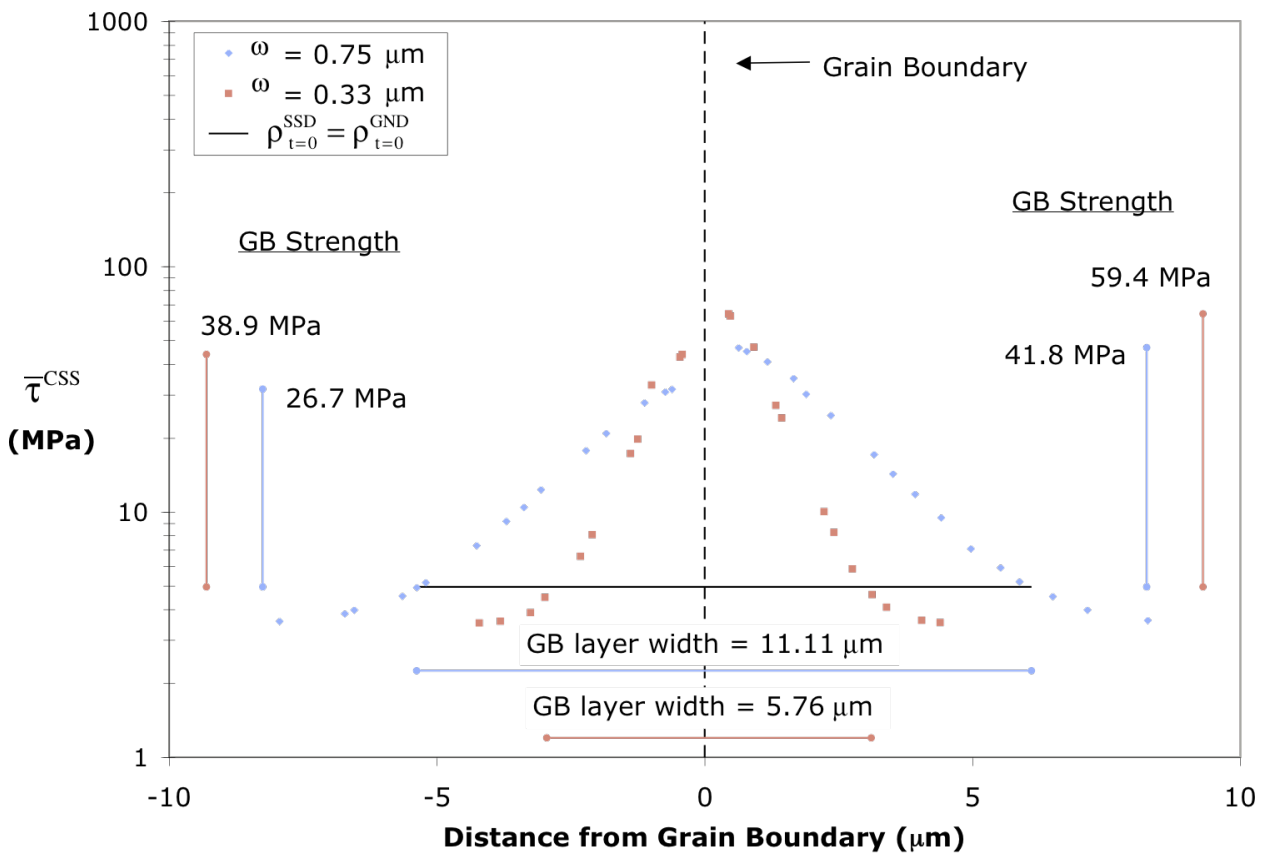

Figure 6.32 - The effect of $\mathrm{C}$ on the GB layer width and GB strength.

As $\omega$ decreases the GB layer width also decreases because the damping rate in the nonlocal integrals has increased. The faster the exponentials approach zero, the more localized the grain boundary effects become. In addition, the GB strength decreases as $\omega$ decreases. This trend is a secondary affect of the slower decay rate. The integrals that make up the denominator in the non-local integral approach are essentially a geometric term that scales with volume. A slower decay rate results in a larger integration volume, and therefore a larger term in the denominator, which in turn lowers the GB strength.

The macroscale affect of both $\mathrm{C}$ and $\omega$ on $\mathrm{n}$ was investigated in Section 6.4.2 and it was found that decreasing $C$ and increasing $\omega$ resulted in a lower $n$ value. These results are now recast in terms of the effect of the material GB layer width and height on $\mathrm{n}$ in Figures 6.33 and 6.34 . 


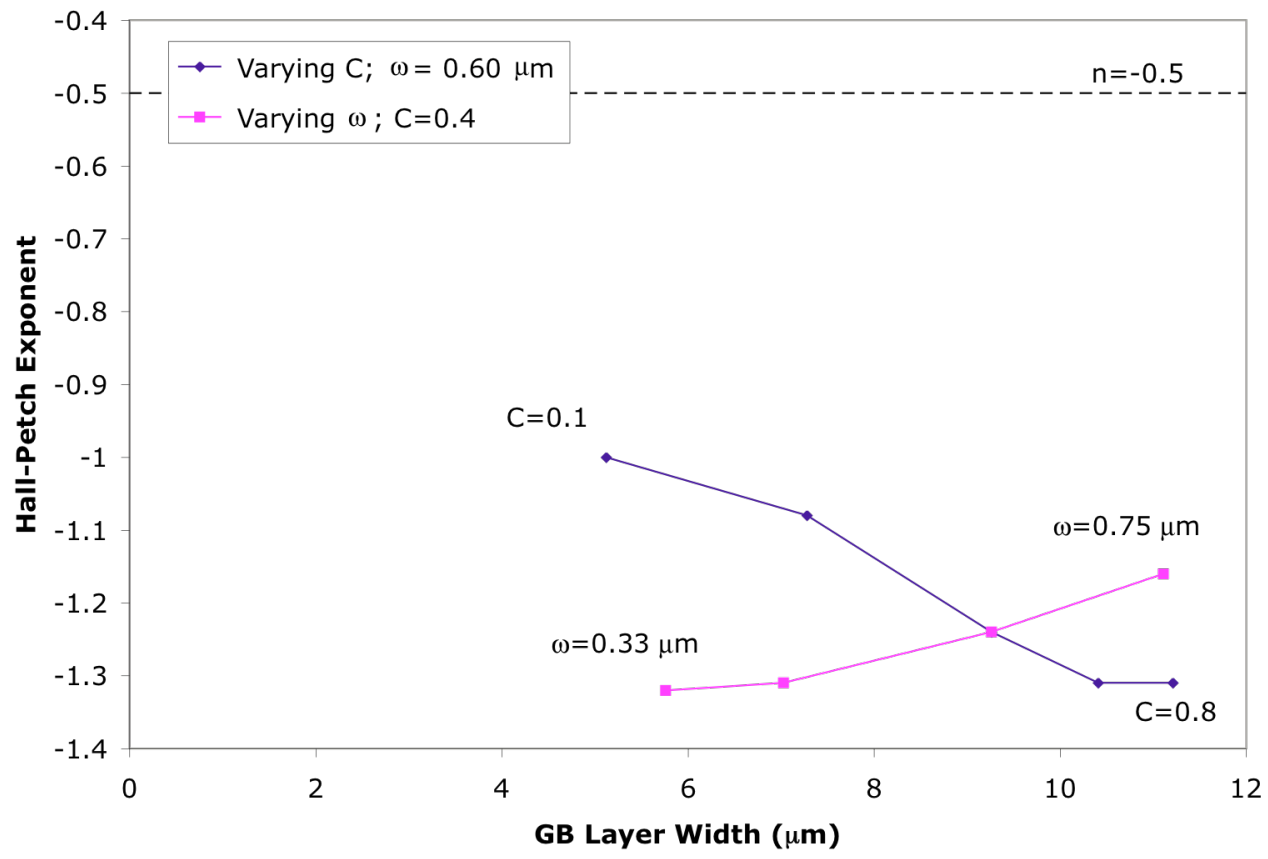

Figure 6.33 - The effect of $\mathrm{C}$ and $\omega$ on the GB layer width

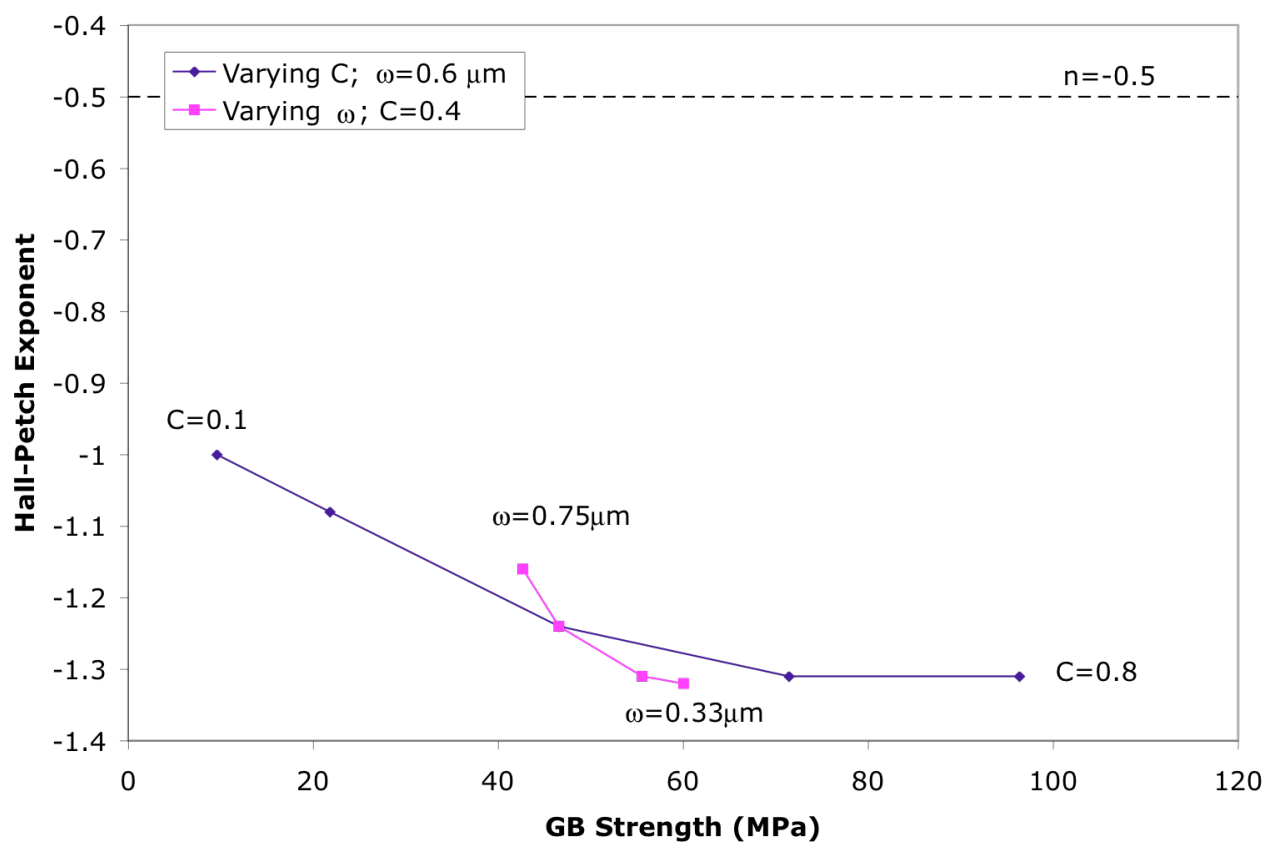

Figure 6.34 - The effect of $C$ and $\omega$ on the GB strength 
Figure 6.33 shows that the Hall-Petch exponent is both directly and inversely related to material GB layer width. Increasing the material GB layer width as a function of $\mathrm{C}$ from $5.12 \mu \mathrm{m}(\mathrm{C}=0.1)$ to $11.21 \mu \mathrm{m}(\mathrm{C}=0.8)$ results in a Hall-Petch exponent increase from -1.0 to -1.31 . Increasing the material GB layer width as a function of $\omega$ from $5.76 \mu \mathrm{m}$ $(\omega=0.33 \mu \mathrm{m})$ to $11.11 \mu \mathrm{m}(\omega=0.75 \mu \mathrm{m})$ results in a Hall-Petch exponent decrease from 1.32 to -1.16 . The problem with these trends is that a consistent correlation between material GB layer width and $\mathrm{n}$ does not exist indicating that the Hall-Petch exponent does not depend on material GB layer width.

The results in Figure 6.34 show that the Hall-Petch exponent is directly related to material GB strength. Increasing the material GB strength as a function of $C$ from 9.6 $\mathrm{MPa}(\mathrm{C}=0.1)$ to $96.3 \mathrm{MPa}(\mathrm{C}=0.8)$ results in a Hall-Petch exponent increase from -1.0 to -1.31. Increasing the material GB strength as a function of $\omega$ from $42.7 \mathrm{MPa}$ $(\omega=0.75 \mu \mathrm{m})$ to $60.1 \mathrm{MPa}(\omega=0.33 \mu \mathrm{m})$ results in a Hall-Petch exponent increase from 1.16 to -1.32 . The consistent trends in Figure 6.34 suggest that the Hall-Petch exponent is a function of GB strength: as the GB strength decreases, the Hall-Petch exponent also decreases.

\subsection{Model Limitations}

In general, the non-local model qualitatively captures the effects of grain boundaries on the stress-strain response of polycrystalline metals. Aside from the $\mathrm{d}^{-1}$ like dependence in the model, there are two other areas where the non-local results do not follow the expected physics. The first problem is related to the effect of misorientation on 
the GB height, and the second problem is associated with the large strain response of the non-local model.

\subsubsection{GB Strength vs. Misorientation}

When the misorientation between two grains is small (less than $10^{\circ}-15^{\circ}$ ), the grain boundary between them is called a low angle grain boundary. The structure of a low angle grain boundary can be represented as an array of dislocations. For example, a symmetrical low angle tilt boundary can be represented as an array of pure edge dislocations as shown in Figure 6.35.

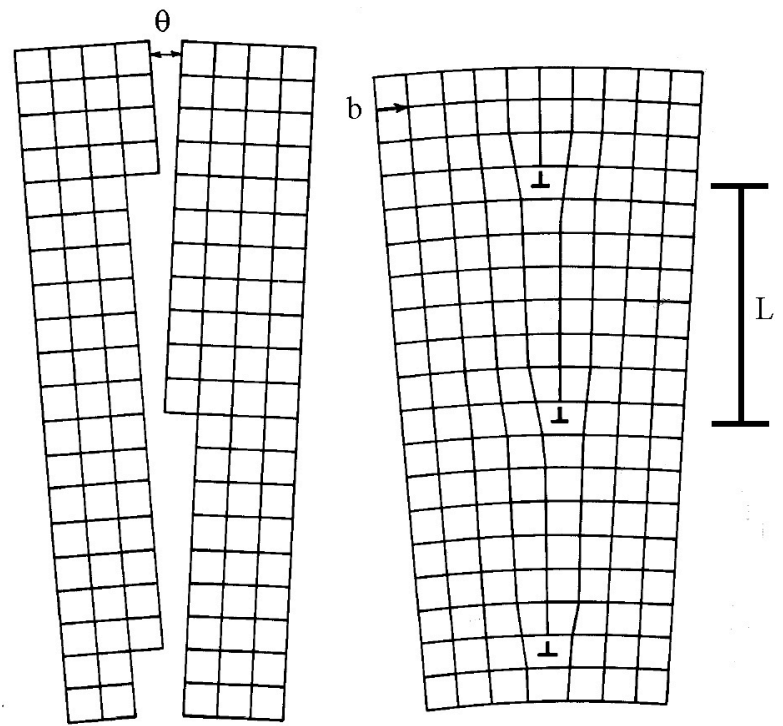

Figure 6.35 - A low angle tilt boundary composed of edge dislocations.

The GND density for a low angle tilt boundary is proportional to the misorientation angle $(\theta)$ between the grains via 


$$
\rho^{\mathrm{GND}} \propto \frac{1}{\mathrm{~L}}=\frac{\sin \theta}{\mathrm{b}} \approx \frac{\theta}{\mathrm{b}}
$$

where $L$ is the dislocation spacing, and $\sin \theta \approx \theta$ when $\theta$ is small. In the non-local model, an increase in $\rho^{\mathrm{GND}}$ (keeping $\mathrm{C}$ and $\omega$ constant) should lead to an increase the GB layer width as shown in Figure 6.36.

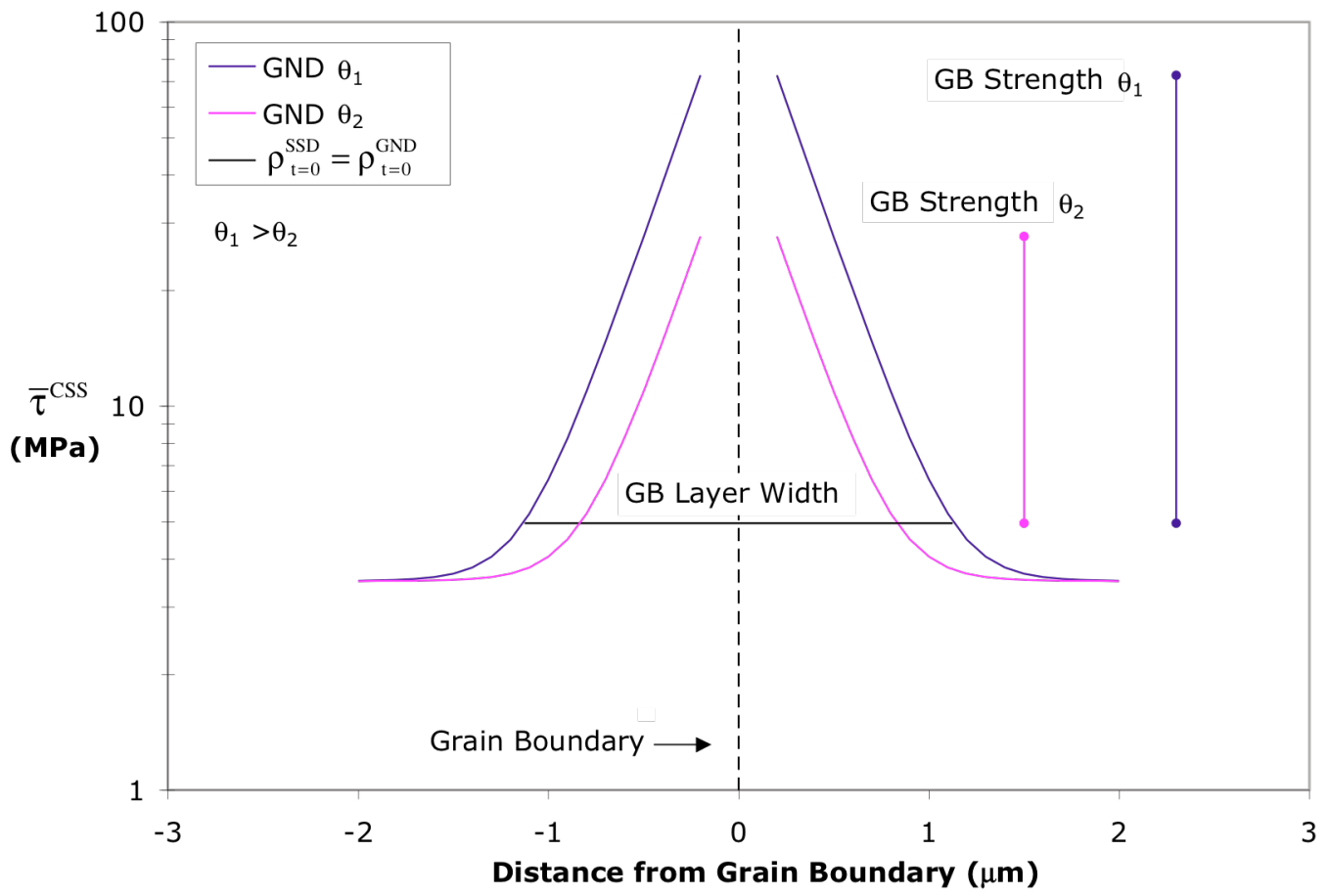

Figure 6.36 - How the change in misorientation leads to a change in GB Strength.

Therefore, Eq. (6.1) suggests that the GB layer width should increase as $\theta$ increases between $0-15^{\circ}$.

It is not clear how the GB length scale should vary when $\theta$ is greater than $15^{\circ}$. The dislocation array model shown in Figure 6.35 breaks down when misorientations get larger than $15^{\circ}$ because the physical dislocation spacing predicted by Eq. (6.1) becomes unphysically small. It is commonly assumed that the grain boundary energy as predicted 
by the Read-Shockley equation is constant (except for special grain boundaries) for high angle grain boundaries, grain boundaries where $\theta>15^{\circ}$. The constant energy assumption for high angle boundaries suggests that $\rho^{\mathrm{GND}}$ might also be constant for high angle boundaries.

The dependence of the GB length scale on $\theta$ was tested by generating grain boundaries with misorientations between $0^{\circ}-45^{\circ}$. In order to generate these misorientations, the grain orientations with the polycrystal were created by rotating between $1^{\circ}-45^{\circ}$ around a constant axis. The GB strength vs. misorientation angle for each of the 48 grain different boundaries in the 16 hex grain polycrystal is plotted in Figure 6.37 .

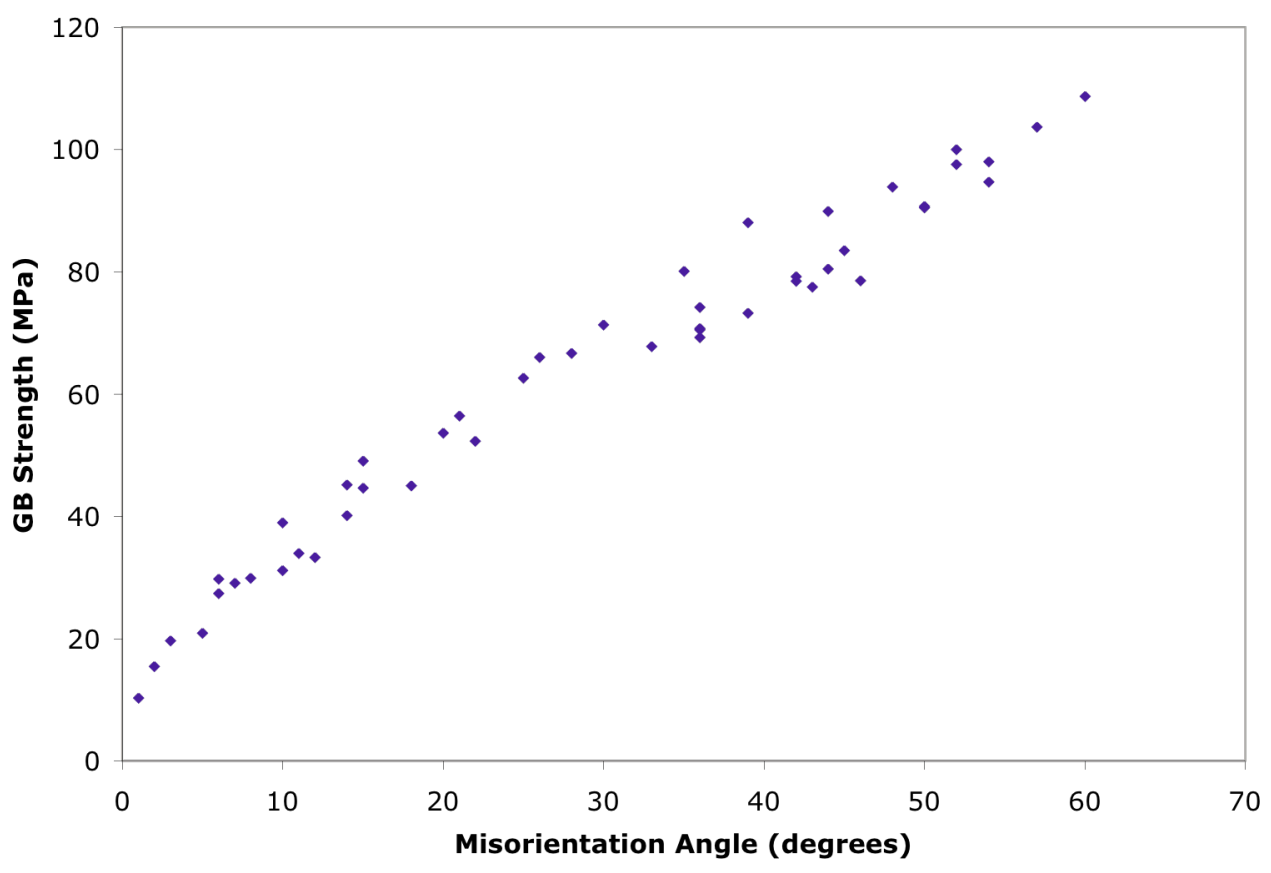

Figure 6.37 - Misorientation angle vs. GB strength. 
For low angle boundaries $\left(\theta<15^{\circ}\right)$ the results in Figure 6.37 look reasonable. The GB height increases (with a little scatter) as misorientation angle increases. After $15^{\circ}$, the GB height continues to increase linearly with misorientation. Unfortunately, the properties of high angle boundaries are not well understood. Thus it is expected that based on a simplistic picture of the GB structure, the height of high angle grain boundaries continue to increase with $\theta$. However, if the energy of high angle grain boundaries is assumed to be constant, then it seems reasonable to think that its structure and properties (i.e., GB strength) are also constant. One possible way to enforce a constant GB strength for high angle grain boundaries is to limit the misorientation between two grains to $15^{\circ}$ during the calculation of $\{\tilde{\mathbf{G}}\}$.

Another possible problem with the current model is the relationship between grain boundary properties and $\theta$. Most experimental works and analytical models use misorientation to characterize grain boundaries, where misorientation is calculated via $\mathbf{R}_{\mathrm{h} \text {-Grain } 1}^{\mathrm{e}} \cdot\left(\mathbf{R}_{\mathrm{h} \text {-Grain } 2}^{\mathrm{e}}\right)^{\mathrm{T}}$. The kinematics on the other hand characterize grain boundaries as a function of Curl $\mathbf{R}_{\mathbf{h}}^{\mathbf{e}}$, which are based on $\mathbf{R}_{\mathrm{h} \text {-Grain } 1}^{\mathrm{e}}-\mathbf{R}_{\mathbf{h} \text {-Grain } 2}^{\mathrm{e}}$. It is important to recognize that the model characterizes grain boundaries differently than experiments and most analytical models. The results in Figure 6.37 seem to indicate that results based on the $\mathbf{R}_{\mathrm{h} \text {-Grain } 1}^{\mathrm{e}}-\mathbf{R}_{\mathrm{h} \text {-Grain2 }}^{\mathbf{e}}$ approach do scale appropriately with misorientation. However it is not clear that such agreement will always be the case. 


\subsubsection{Large Strain Response Predicted by the Non-Local Model}

In order to generate yield strength data, there was little need to run the non-local model to large strains. Yield strength simulations were run out to $0.5 \%$ strain, and the resulting stress-strain curves for different grain sizes were stacked on top of each other, consistent with experimental observations (See Figures 6.12 and 6.13). Experimentally, the stress strain curves for different grain sizes remain parallel to large strains, indicating that the rate of work hardening is independent of grain size. Unfortunately, this trend is not seen at large strains in the non-local simulations as shown in Figure 6.38.

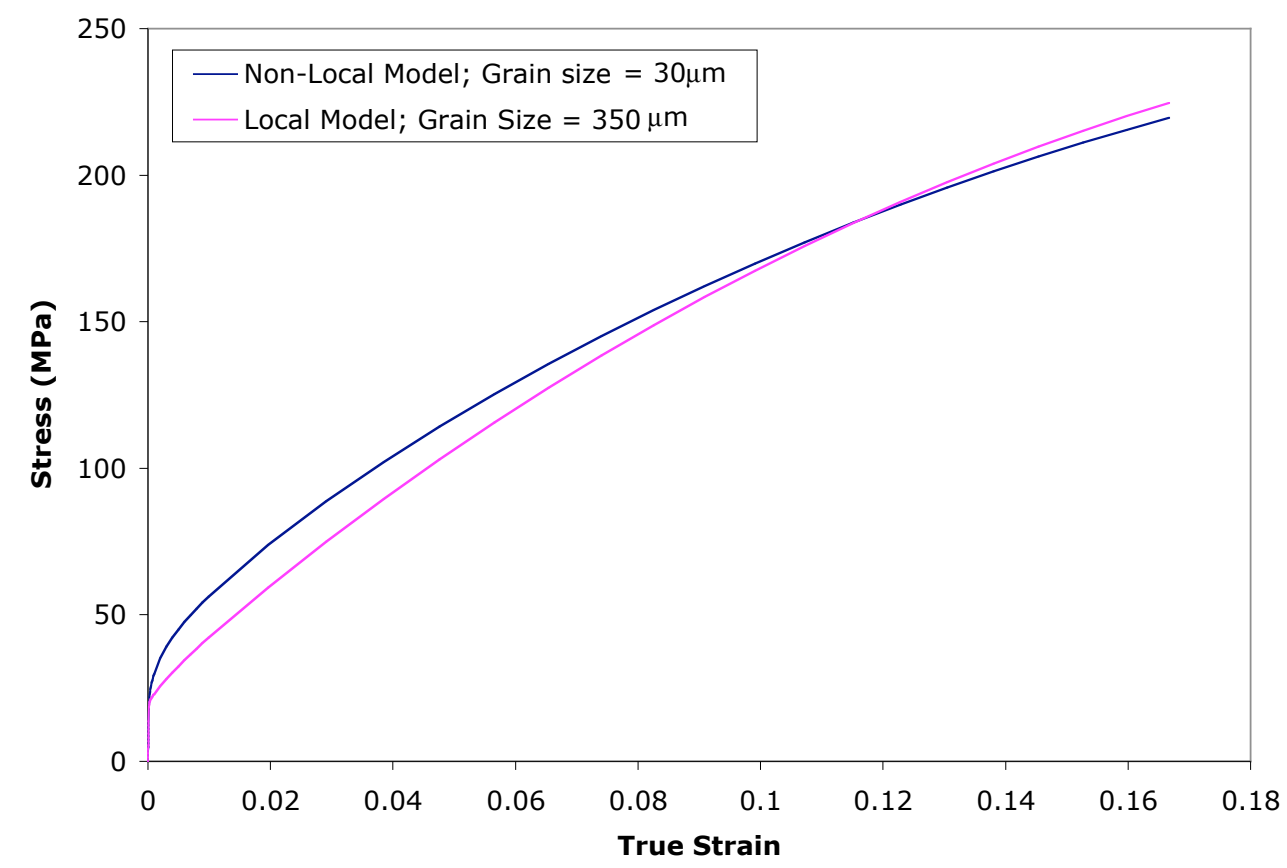

Figure 6.38 - Stress-strain results from the local and non-local models out to $17 \%$ strain.

The reason for the curve crossing between the local and non-local models is not clear at this time. 


\section{CHAPTER 7}

\section{INVESTIGATION OF INTRAGRAIN MISORIENTATIONS IN POLYCRYSTALLINE NICKEL}

In Chapter 6, macroscale experimental results, such as tensile stress-strain curves, were used to validate the macroscale results of the non-local model. These macroscale results depend on parameters that describe microscale features (like $\rho^{\mathrm{SSD}}$ and $\rho^{\mathrm{GND}}$ ). In Chapter 7, microscale simulation results, misorientation maps, are compared with microscale experimental results. It is important to examine microscale results in order to determine whether the microscale parameters used to achieve the appropriate macroscale response represent the relevant physics.

Brewer et al. (2006) experimentally investigated the change in grain orientation in polycrystalline nickel due to an applied tensile load. This change was tracked by taking Electron Back Scatter Diffraction (EBSD) data from the same location at 0\%, 1\%, 5\%, and $10 \%$ strain. The starting microstructure at $0 \%$ strain was then used as the initial microstructure for tensile test simulations using both the local and non-local crystal plasticity models. The simulated change in grain orientations was then compared with experimental results on the same microstructure.

\subsection{Experimental Methods}

Nickel tensile specimens were made from sheet material with a thickness of $1.6 \mathrm{~mm}$ and a nominal purity of $99.9 \%$. The sheet material was annealed at $1071^{\circ} \mathrm{C}$ for 30 minutes under flowing argon and then sectioned into tensile specimens using electro- 
discharge machining (EDM). Each specimen was $10 \mathrm{~cm}$ long with the other dimensions conforming to the appropriate ASTM standard (ASTM E8-04). In order to make EBSD measurements, one surface of each tensile specimen was polished to a $0.05 \mu \mathrm{m}$ finish with diamond polishing compounds and colloidal silica.

A series of Vickers indentations on the polished surface marked the area from which EBSD data would be taken. The analysis areas were at least $280 \mathrm{~mm}$ away from the marker indents. EBSD data was taken from a $250 \mu \mathrm{m} \times 250 \mu \mathrm{m}$ area with a step size of $0.5 \mu \mathrm{m}$. The EBSD measurements were performed using a Zeiss Supra 55 VP-FEG SEM. The measurements were taken at $20 \mathrm{keV}$. A Nordlys CCD camera was used to collect the diffraction patterns on this system. The acquisition and analysis of the EBSD data was performed with the HKL Channel 5 software suite.

Tensile tests were conducted on an MTS servo-hydraulic test frame at rate of $0.0005 \mathrm{in} / \mathrm{sec}$. Strains in the gauge section were measured using an MTS clip gauge extensometer. The nickel tensile specimen was deformed to $1 \%, 5 \%$, and $10 \%$ strain in separate tensile tests. At each strain level, grain orientation data collected from EBSD was used to calculate local intragrain misorientation (LIMIS) and average intragrain misorientation (AMIS). Details on these misorientation calculations are contained in Appendix D and Brewer et al. 2006. 


\subsection{Experimental Results}

The initial grain orientations in the area of interest were determined with EBSD prior to any tensile testing. The orientations for the $250 \mu \mathrm{m} \times 250 \mu \mathrm{m}$ area of interest are displayed in the inverse pole figure (IPF) map shown in Figure 7.1.
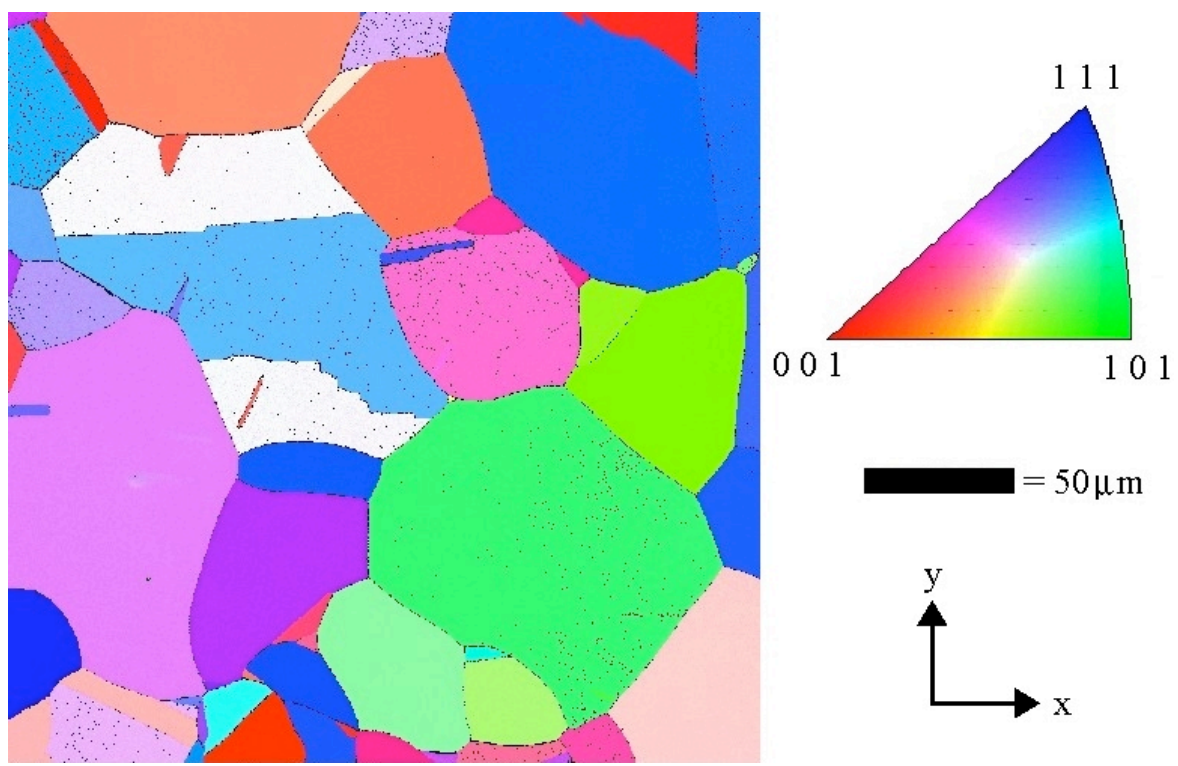

Figure 7.1 - An inverse pole figure map along the loading $(\mathrm{X})$ axis.

The IPF map reveals that the microstructure is composed of relatively large grains and much smaller annealing twins. Such a microstructure is typical of polycrystalline nickel. Grain size measurements were made on this field of view as well as 2 others using a $10^{\circ}$ grain boundary criterion and the line intercept method. The resulting average grain size was $31 \mu \mathrm{m}$.

In reference to the coordinate axis defined in Figure 7.1, the tensile tests were performed along the $\mathrm{x}$ direction. A composite stress-strain curve of the three individual 
tensile tests was put together by setting the strain at the beginning of the $1 \%$ and $5 \%$ tensile tests equal the strain at the end of the previous tensile test. This composite stressstrain curve is shown in Figure 7.2.

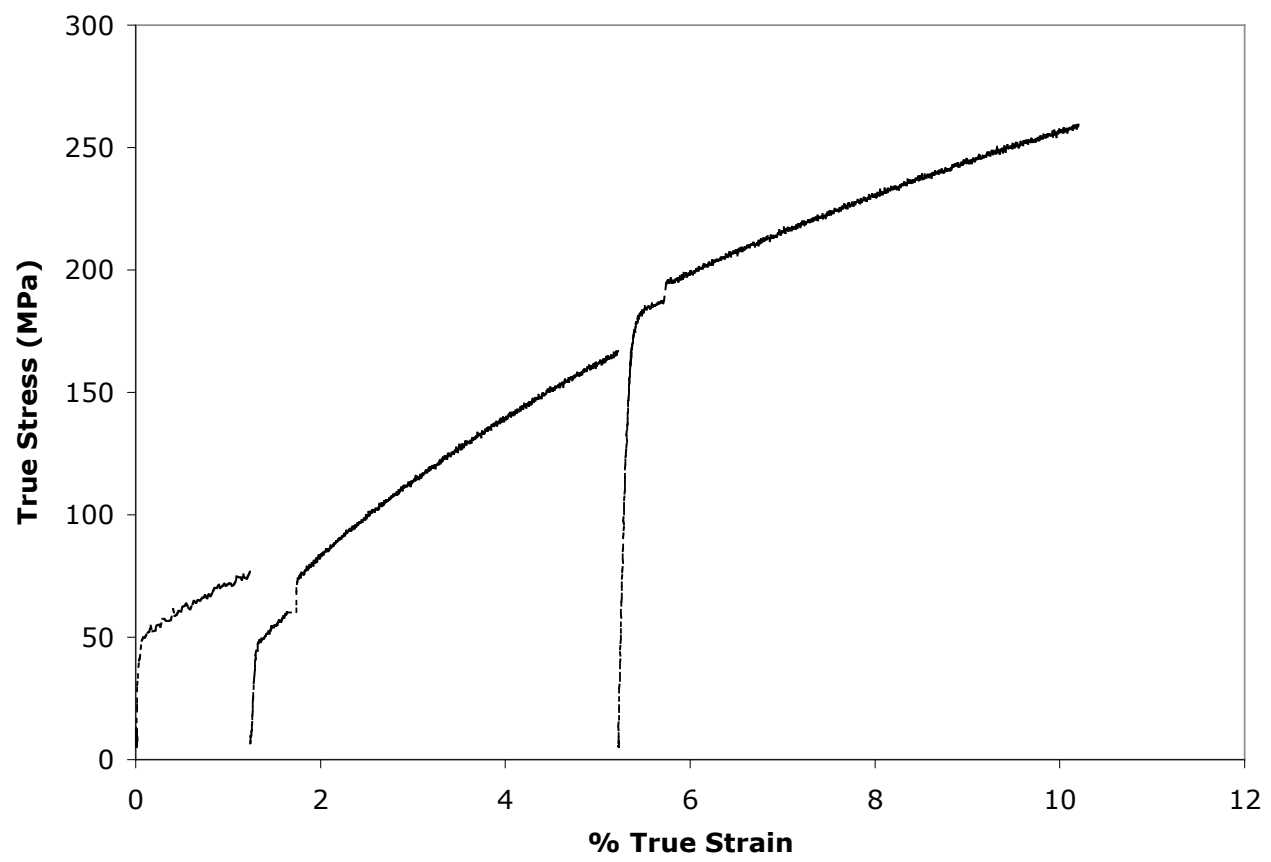

Figure 7.2 - Composite stress-strain curve for the nickel tensile specimen

IPF maps from the same area shown in Figure 7.1 were taken at $1 \%, 5 \%$, and $10 \%$ strain. These IPF maps are shown in Figure 7.3. 


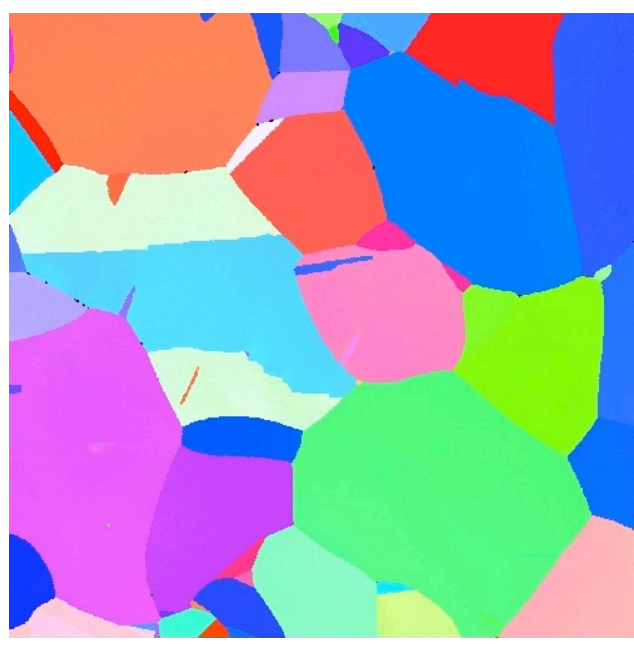

(a) $1 \%$ strain

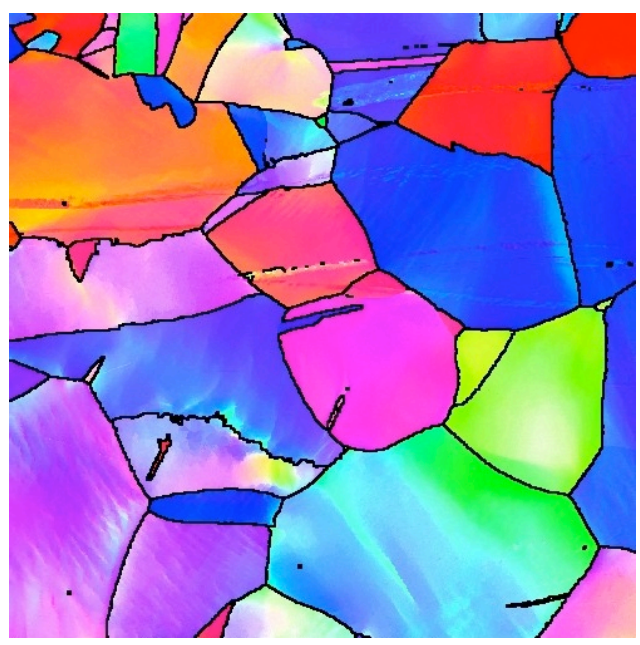

(c) $10 \%$ strain

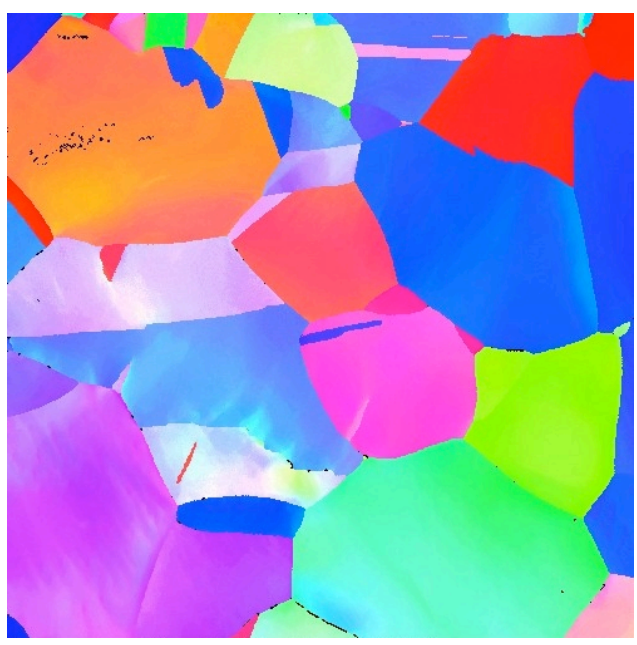

(b) $5 \%$ strain
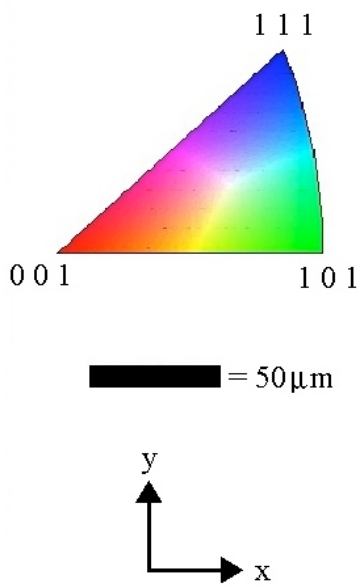

Figure 7.3 - Inverse pole figure maps at 1\%, 5\%, and $10 \%$ strain. 
The IPF map at $1 \%$ strain is similar to the initial IPF map (Figure 7.1) indicating there was little orientation change. However, the IPF maps at 5\% and $10 \%$ strain do show noticeable changes in internal grain orientation when compared to the initial IPF map. These EBSD micrographs in Figure 7.3 were then used to calculate LIMIS and AMIS. The misorientation results are presented in Section 7.4 as comparisons to simulation results.

\subsection{Simulation Parameters}

The simulation parameter needed to run the local and non-local models were obtained mainly from the initial inverse pole figure map (Figure 7.1) and the composite stress-strain curve (Figure 7.2). A few other parameters were taken from the literature. Topics included in this section are the following: 1) Initial Microstructure, 2) Elastic and Viscoplastic Constants, 3) Hardening Constants.

\subsubsection{Initial Microstructure and Boundary Conditions}

The EBSD data at $0 \%$ strain represents the initial orientation state of the grains within the polycrystal making the initial orientation state a known quantity. The angular orientation resolution on the EBSD data was $1.0^{\circ}$, meaning that the raw EBSD data was sensitive to small misorientations within the grain. It is not computationally efficient to account for all these small misorientations within a grain nor will these small misorientations greatly affect the results. Thus, 3 average Euler angles for each grain 
were determined and these 3 average Euler angles represented the initial orientation of each grain used in the simulations.

The mesh of the initial microstructure was generated by making each pixel in the initial inverse pole figure map an element. With this approach, the mesh contained 250,000 elements, which was too large for the non-local model. Thus, the mesh was reduced to 27,556 elements by taking every $3^{\text {rd }}$ element and every $3^{\text {rd }}$ line. This reduced mesh is shown in Figure 7.4.

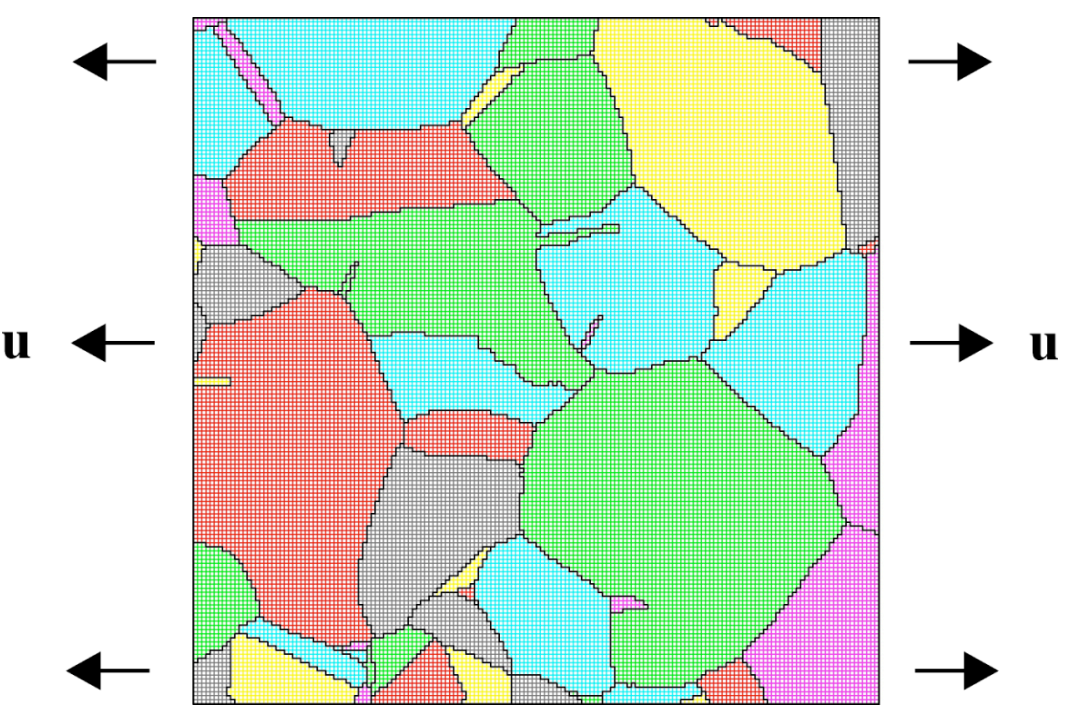

Figure 7.4 - The initial meshed microstructure and the applied displacement boundary conditions.

The mesh in Figure 7.4 was comprised of eight node (hexahedral) 3D isoparametric elements with a single integration point at the element centroid. In addition, the mesh was " $2 \mathrm{D}+1$ ", which means it is only one element thick in the third dimension. Unlike any of the polycrystalline meshes used in the previous chapter, PBC cannot be used in the $\mathrm{x}$ and $\mathrm{y}$ directions of this mesh. 
FEM simulations of a displacement controlled tensile test were performed on the meshed microstructure, as shown in Figure 7.4. Because the applied displacements were balanced, the material body was in equilibrium. The applied displacements were constrained to the $\mathrm{x}$ direction. Thus the left and right plane could only move in the $\mathrm{x}$ direction. There was no displacement or force was applied to either the top or the bottom, which allowed them to deform freely. PBCs were used in the z-direction. A no flux boundary condition was used for the integral quantity. Numerically, this was accomplished by using FSBC \#1 (Section 6.1.6.1) on the 4 surfaces in Figure 7.4.

\subsubsection{Elastic and Viscoplastic Parameters}

The burgers vector and anisotropic elastic constants $\left(L_{11}, L_{12}\right.$, and $\left.L_{44}\right)$ for bulk polycrystalline copper were taken from the literature (Hertzberg, 1983). In addition, the two viscoplastic flow rule parameters, $\dot{\gamma}_{0}$ and $\mathrm{m}$, were set to typical literature values. All of these constants are listed in Table 7.1.

Table 7.1 - Elastic and viscoplastic parameters used in nickel simulations

\begin{tabular}{|c|c|c|c|c|c|c|}
\hline & $L_{11}(\mathrm{GPa})$ & $L_{12}(\mathrm{GPa})$ & $L_{44}(\mathrm{GPa})$ & $\mathrm{b}(\mathrm{A})$ & $\dot{\gamma}_{0}(1 / \mathrm{sec})$ & $\mathrm{m}$ \\
\hline Nickel & 246.5 & 147.3 & 124.7 & 2.517 & 1 & 25 \\
\hline
\end{tabular}

\subsubsection{Hardening and Non-Local Parameters}

Generally, all three of the hardening parameters are determined by fitting a length scale independent stress-strain curve (i.e., a stress-strain curve for a grain size whose 
yield strength is grain size independent) with the local crystal plasticity model, as was done Section 6.1.3. In this case, such a stress-strain curve does not exist. Therefore, the nickel hardening parameters were determined two steps.

First, the hardening parameters $\mathrm{c}_{\mathrm{KM} 1}$ and $\mathrm{c}_{\mathrm{KM} 2}$ were determined by fitting the local crystal plasticity model to the composite stress-strain curve shown in Figure 7.2.

Discontinuities in the composite stress-strain curve appear when the nickel sample was reloaded at $1 \%$ and $5 \%$ strain making an exact fit impossible. In the final fit, shown in Figure 7.5 as Local Model-30 $\mu \mathrm{m}$, priority was given to matching the 5-10\% strain region of the composite stress-strain curve.

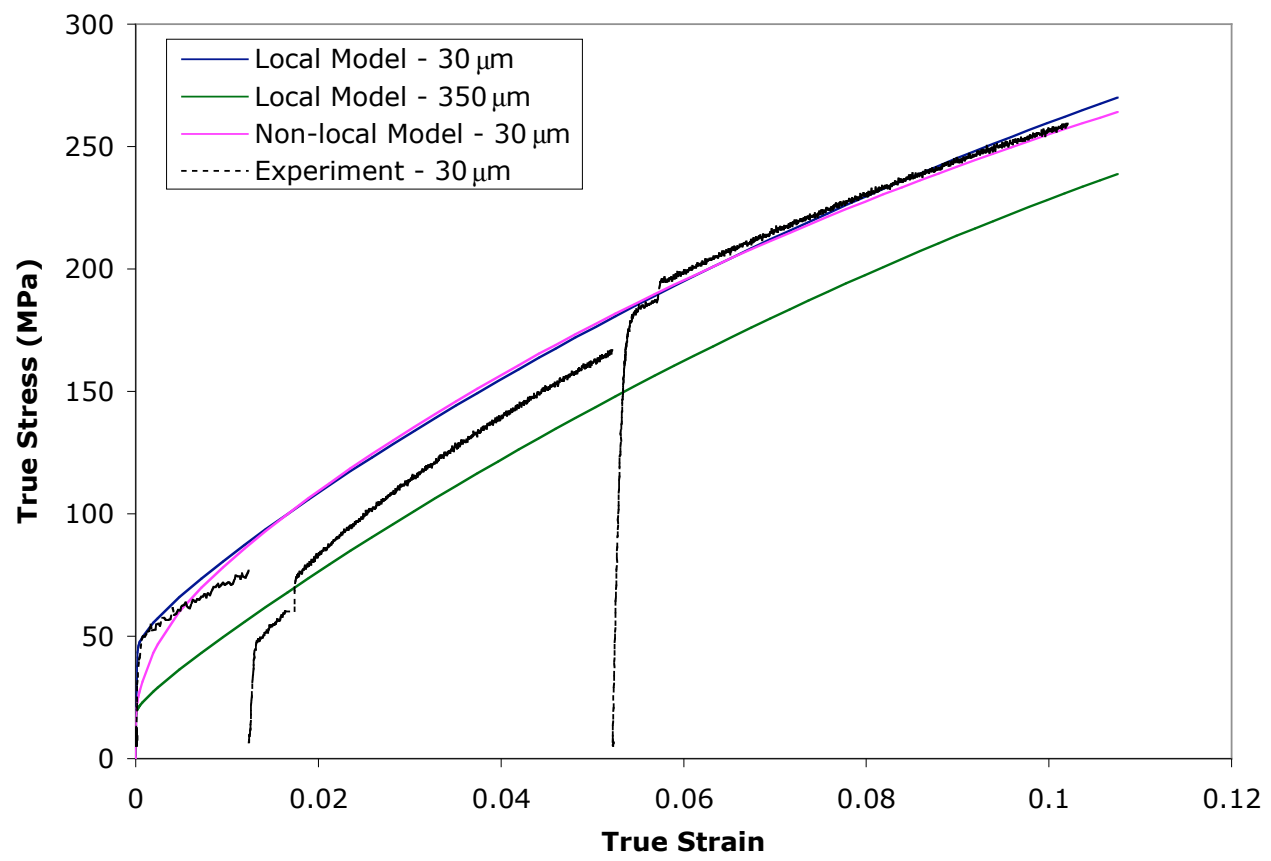

Figure 7.5 - Local and non-local model fit to the experimental stress-strain curve.

Next, the missing length scale independent stress-strain curve was generated.

This curve is a necessary base-line from which the non-local model can predict grain size 
effects. The length scale independent stress-strain curve was generated with the previously determined hardening parameters $\mathrm{c}_{\mathrm{KM} 1}$ and $\mathrm{c}_{\mathrm{KM} 2}$ as well as a literature value for the $\sigma_{\mathrm{ys}}$ of a nickel polycrystal with a $350 \mu \mathrm{m}$ grain size. The literature $\sigma_{\mathrm{ys}}$ value was determined from Hansen's (2004) fit of Thompson's (1975) experimental Hall-Petch data: $\sigma_{0}=20 \mathrm{MPa} ; \mathrm{k}=0.16 \mathrm{MPa} \mathrm{m}^{-0.5} ; \mathrm{n}=-0.5$. With these constants, $\sigma_{\mathrm{ys}}$ at $\mathrm{d}=350 \mu \mathrm{m}$ is 28.6 MPa. The local model was then used to generate a length scale independent stressstrain curve with a yield strength of $28.1 \mathrm{MPa}$, shown in Figure 7.5 as Local Model $350 \mu \mathrm{m}$. The hardening parameters for both local model simulations are shown in Table 7.2.

Table 7.2 - Hardening parameters used in nickel simulations

\begin{tabular}{|c|c|c|c|c|}
\hline Material & Grain Size & $\tau_{\mathrm{t}=0}^{\mathrm{CSS}}(\mathrm{MPa})$ & $\mathrm{c}_{\mathrm{KM} 1}(1 / \mathrm{m})$ & $\mathrm{c}_{\mathrm{KM} 2}$ \\
\hline Nickel & $31 \mu \mathrm{m}$ & 19.1 & $3.606 \times 10^{8}$ & 26.65 \\
\hline Nickel & $\approx 350 \mu \mathrm{m}$ & 8.0 & $3.606 \times 10^{8}$ & 26.65 \\
\hline
\end{tabular}

The non-local model was then fit to the experimental composite stress-strain curve with the hardening parameters for the $\approx 350 \mu \mathrm{m}$ grain size polycrystal by adjusting $\mathrm{C}$ and $\omega$. The $\mathrm{C}$ and $\omega$ parameters used to generate the best curve shown in Figure 7.4 as

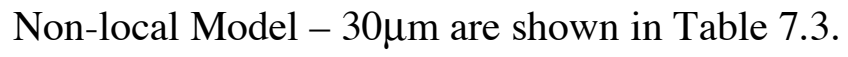

Table 7.3 - Non-local parameters used in nickel simulations

\begin{tabular}{|c|c|c|c|}
\hline Material & Grain Size & C & $\omega(\mu \mathrm{m})$ \\
\hline Nickel & $31 \mu \mathrm{m}$ & 0.5 & 0.4 \\
\hline
\end{tabular}




\subsection{Simulation Results}

From the experimental IPF maps in Figure 7.3, both LIMIS and AMIS were calculated at $1 \%, 5 \%$, and $10 \%$ strain. LIMIS and AMIS were also calculated from the local and non-local simulation results of the polycrystal with a $30 \mu \mathrm{m}$ grain size. These simulation results were then compared with the experimental results.

It should be noted that the experimental results were taken from a free surface, while the simulation results, due the PBCs in all three directions, represent an area in the bulk of the material. There is some experimental evidence that grains at the surface are able to rotate more than grains in the bulk because grains at the surface are less constrained (Barabash et al., 2005). However, it is not clear to what degree grains in the bulk rotate less than surface grains. Regardless, the general misorientation trends observed at the surface are most likely not too different than those in the bulk making the comparison between simulation and experiment relevant.

The LIMIS measurement quantifies the spatial misorientation distribution at a given strain level within each grain. Each LIMIS calculation is based on making the minimum misorientation within a grain a baseline value from which all other misorientations within that grain are determined. So there is a zero LIMIS value within each grain regardless of how much misorientation has accumulated. It must also be stresses that the LMIS calculation is done intragrain meaning the misorientation across grain boundaries does not affect the LIMIS calculation. AMIS, on the other hand, quantifies the amount of misorientation within each grain. 
At $1 \%$ strain, there is little orientation change in either the experiments or simulations as seen in Figure 7.6.

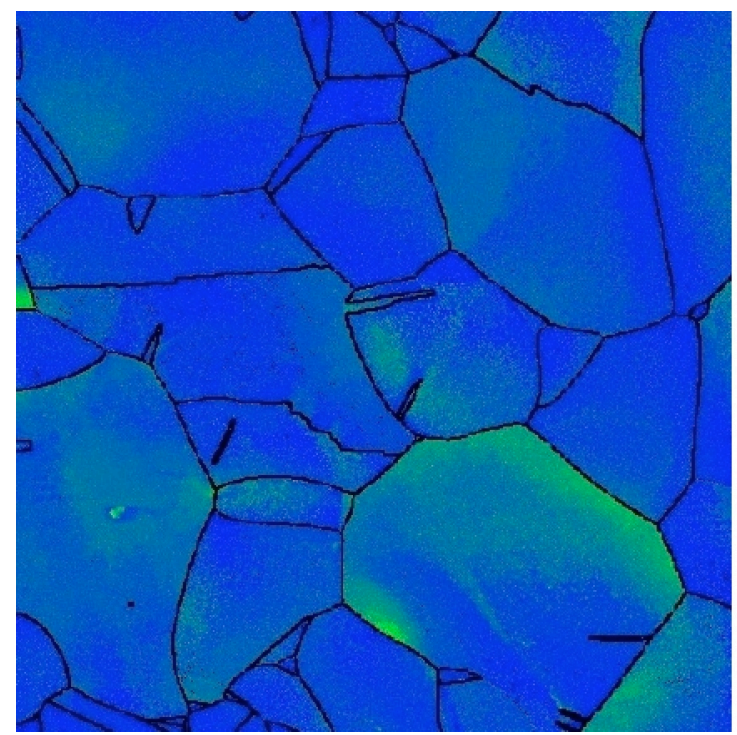

\section{LIMIS}

(Degrees)

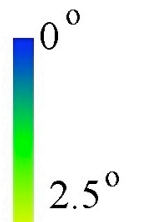

(a) Experimental Results

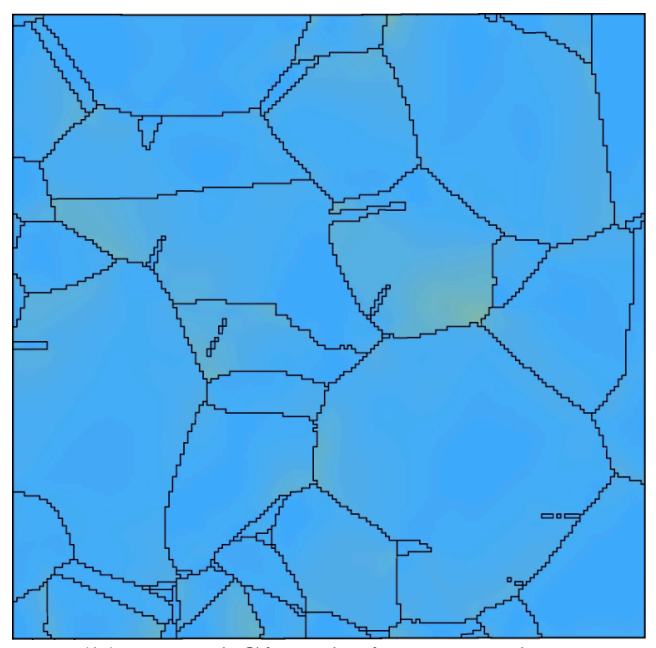

(b) Local Simulation Results

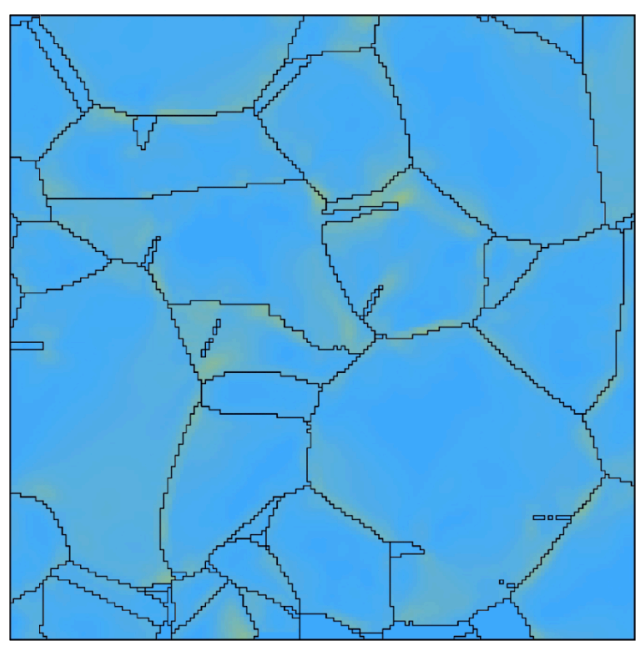

(c) Non-Local Simulation Results

Figure 7.6 - Local intragrain misorientation maps at $1 \%$ strain.

In both the simulation and the experiments, the maximum LIMIS is about $2^{\circ}$, and the maximum LIMIS is observed at the grain boundaries. 
At $5 \%$ and $10 \%$, an appreciable amount of LIMIS has developed in the experiments and the simulations, as seen in Figures 7.7 and 7.8. The LMIS in the experimental results at $5 \%$ and $10 \%$ strain is

- Greatest at the grain boundary,

- Extends into the grain from grain boundaries,

- Often occurs only on one side of the grain boundary.

At $5 \%$ strain most of the LMIS is around $5^{\circ}$, and at $10 \%$ strain it is around $10^{\circ}$. Neither the local nor the non-local models predict enough LIMIS. The maximum LIMIS predicted by the local model was $2.96^{\circ}$ at $5 \%$ strain and $6.46^{\circ}$ at $10 \%$ strain. The maximum LIMIS predicted by the non-local model was $4.52^{\circ}$ at $5 \%$ strain and $7.12^{\circ}$ at $10 \%$ strain. Quantitatively, the local and non-local crystal plasticity models under predict LIMIS. 


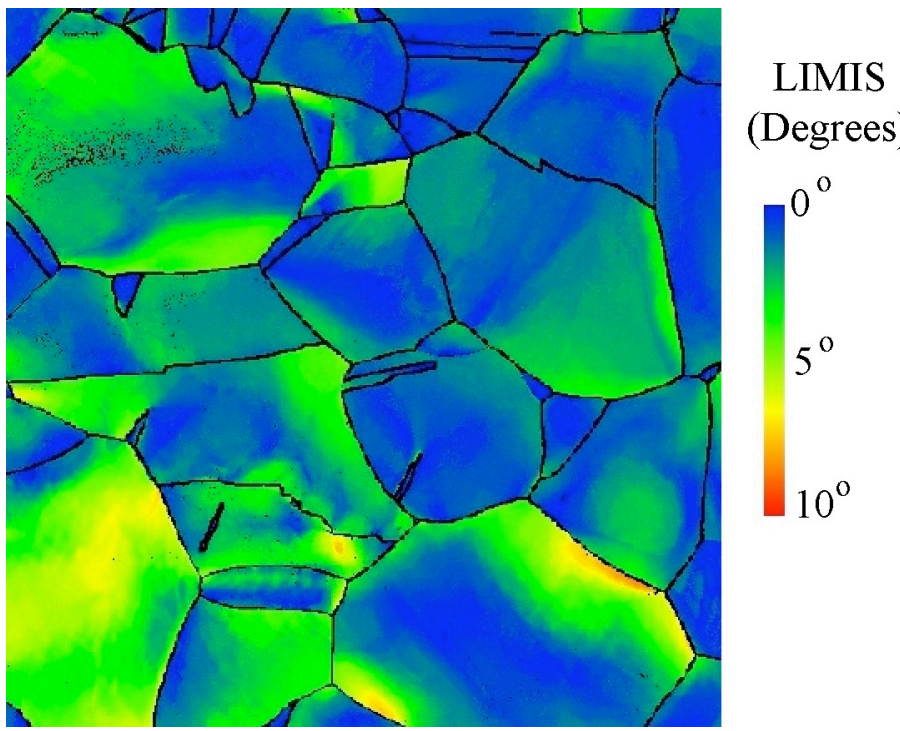

(a) Experimental Results

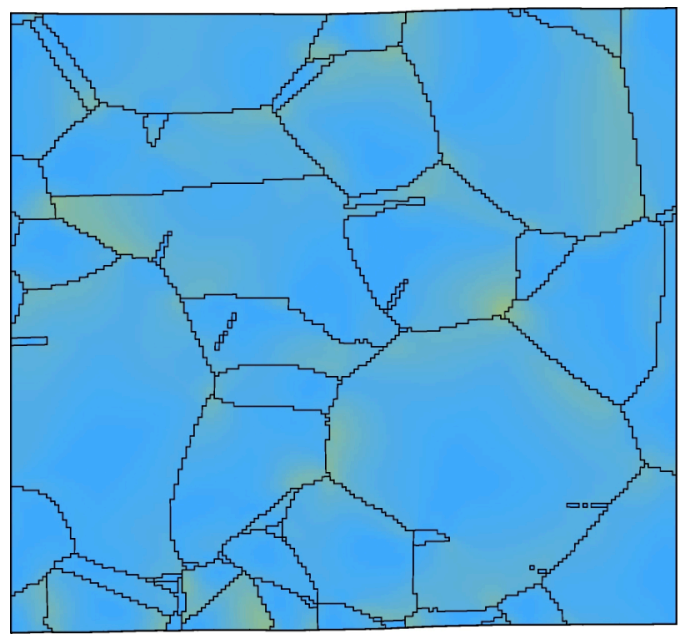

(b) Local Simulation Results

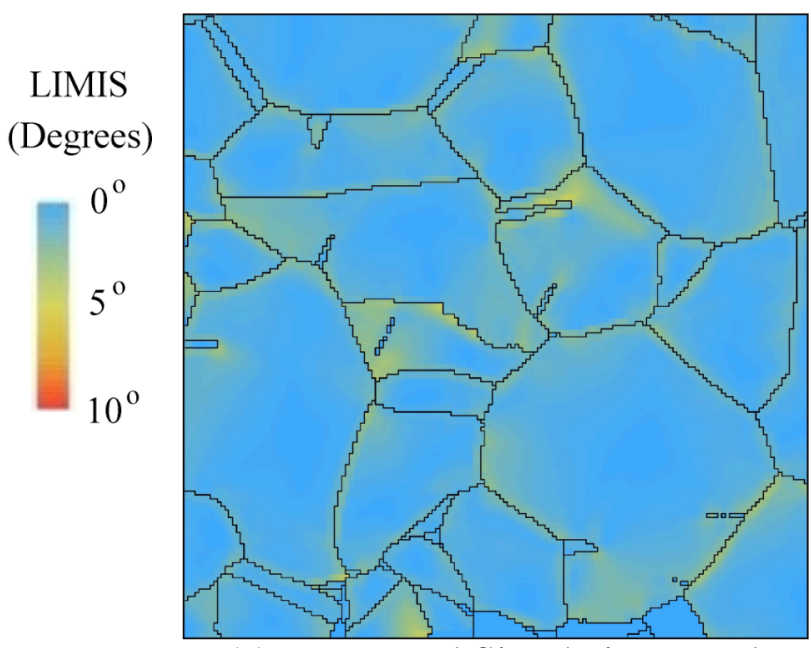

(c) Non-Local Simulation Results

Figure 7.7 - Local intragrain misorientation maps at 5\% strain. 


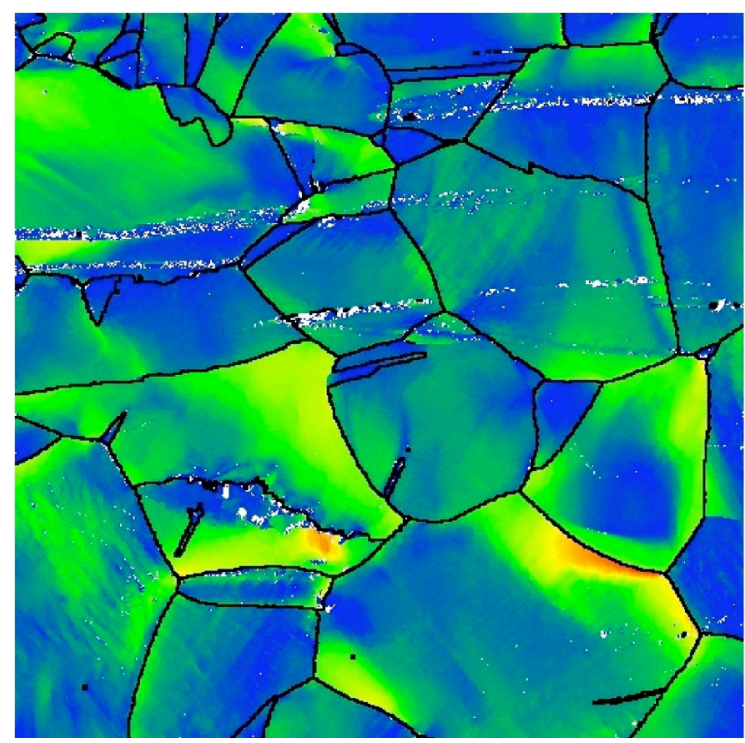

LIMIS

(Degrees)

(a) Experimental Results

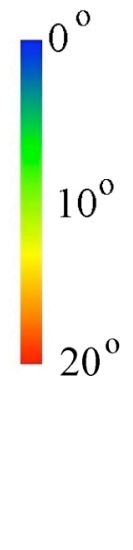

$10^{\circ}$

$20^{\circ}$

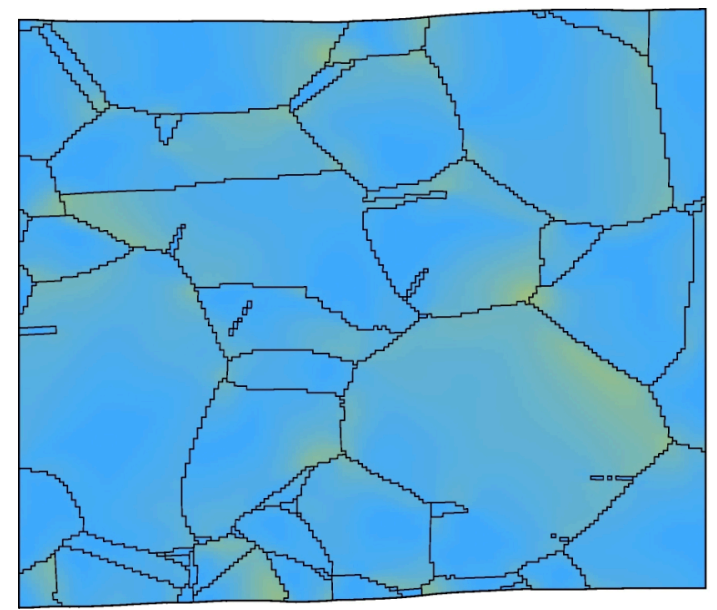

(b) Local Simulation Results

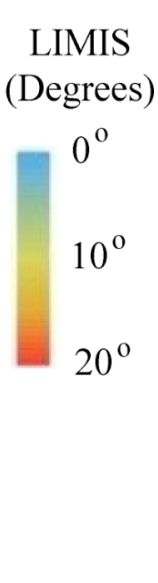

(c) Non-Local Simulation Results

Figure 7.8 - Local intragrain misorientation maps at $10 \%$ strain. 
In order to get investigate the qualitative LIMIS trends, the local and non-local simulation results were replotted with scales appropriate to each result as shown in Figures 7.9 and 7.10. In general, the trends in the simulation results match those observed experimentally. The LIMS is greatest at the grain boundary and it extends into the grain from these points at the grain boundaries. In the results from the non-local model, the grain boundary region always has an elevated LIMIS value, a trend not observed experimentally. Qualitatively, both the local and non-local models do an adequate job of predicting LIMIS even though the non-local model over states the effect at grain boundaries. 


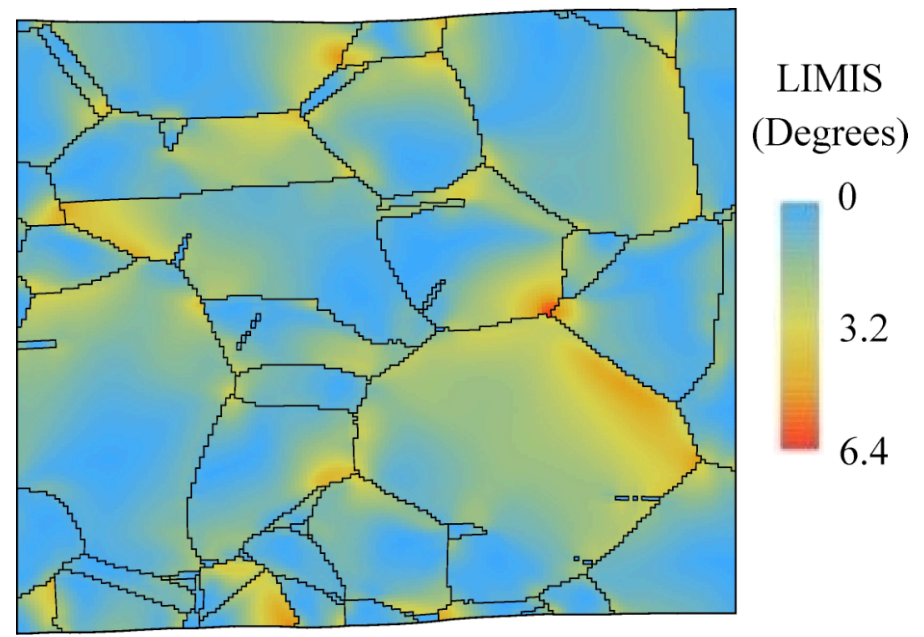

Figure 7.9 - Local LIMIS results at 10\% strain.

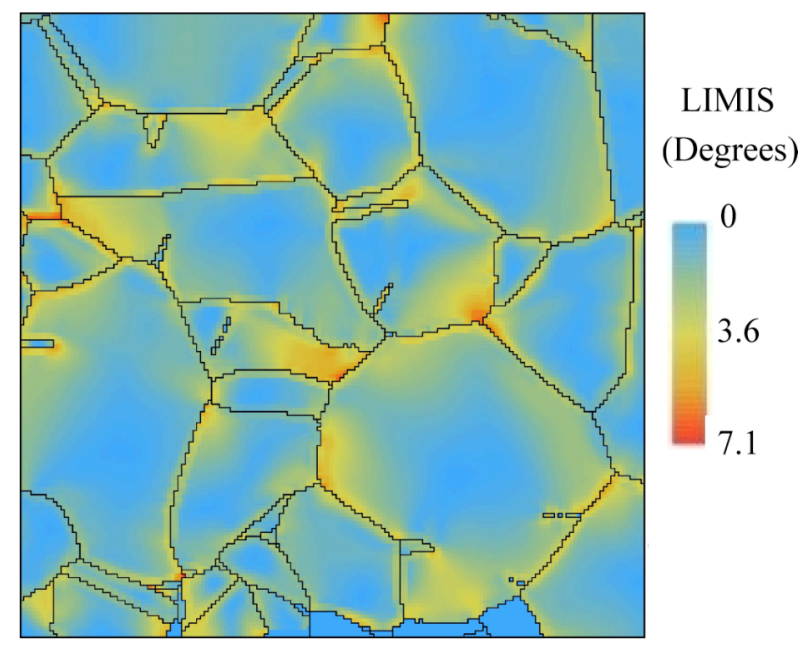

Figure 7.10 - Non-local LIMIS results at 10\% strain. 
The presence of an elevated LIMIS value at most grain boundaries is due to hardening the grain boundary regions. In the crystal plasticity models, the change in orientation is calculated from the quantity $\mathbf{R}^{\mathbf{e}}$. Because plastic flow is suppressed in the hardened grain boundary regions, $\mathbf{F}^{\mathrm{e}}$ (and consequently $\mathbf{R}^{\mathrm{e}}$ ) evolves rather than $\mathbf{F}^{\mathbf{P}}$ in these hardened regions. Therefore, the non-local model will predict more LIMIS at grain boundaries.

The average misorientation (AMIS) within each grain was also calculated from the experimental and simulation results. These AMIS results are shown in Figure 7.11.

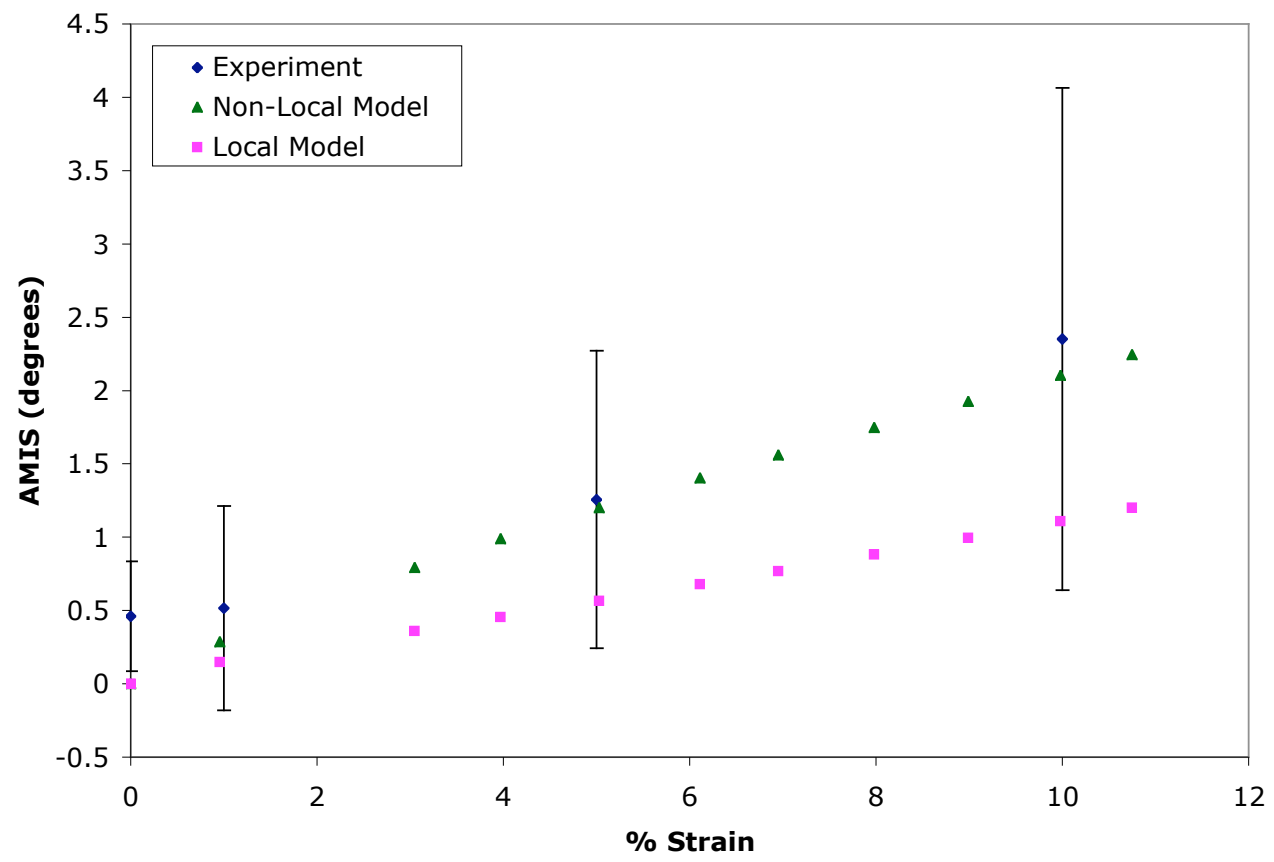

Figure 7.11 - Comparison of experimental, local simulation, and non-local simulation AMIS results.

The error bars on the experimental values are large because the field of view used to make the AMIS measurement had a relatively small number of grains (approximately 60 grains). Increasing the field of view and the number of grains used in the AMIS analysis 
will decrease the size of the error bars. Unfortunately, size limitations on the simulations restrict the size of the field of view.

The experimental AMIS value at $0 \%$ is about 0.5 , which is due to the initial misorientations within each grain. Both the simulations have a 0 AMIS value at $0 \%$ strain because the initial misorientations with each grain were averaged out to get a single orientation for each grain. As the material is strained, all three AMIS values increase linearly. A linear relation between AMIS and strain has been experimentally observed in a number of different materials (Fukuoka et al, 2002; Young et al., 2002). The local model under predicts the experimental AMIS values indicating that the local model does not predict enough overall orientation change within each grain. The non-local model does a good job predicting the experimental AMIS values meaning that the non-local model does sufficiently predict overall orientation change within each grain.

Considering both the LIMIS and the AMIS results together provides an overall picture of the microscale. The local model under predicts both LIMIS and AMIS. These results suggest that the local model predicts a spatial misorientation distribution that is too uniform (LIMIS) and on average there is not enough intragrain misorientation (AMIS). The non-local model, on the other hand, under predicts the LIMIS but does correctly predict AMIS. In this case, the non-local model predicts a spatial misorientation distribution that is too uniform, but on average has the correct amount of misorientation. These microscale results illustrate that neither the local nor the non-local crystal plasticity models correctly accounts for the spatial details at the microscale. However, in terms of microscale averages the non-local model is an improvement over the local model. 


\section{CHAPTER 8}

\section{CONCLUSIONS}

The research project reported in this dissertation has addressed modeling of length scale dependent phenomena experimentally that have been observed in polycrystalline metallic materials during plastic deformation. Past experiments have shown that specimen dimensions and microstrucutral features can influence the mechanical properties of single crystal and polycrystal metallic materials when the dominant length scale is less than a couple hundred microns (Hall, 1951; Petch, 1953; Brenner, 1956; Fleck et al., 1994; Stolken and Evan, 1998; Uchic, 2004). The need to model these length scale dependent phenomena is becoming more and more important as physical dimensions and/or microstrucutral feature sizes in components approach the micron size scale such as in thin films, microelectromechanical systems (MEMS), electronic packaging, and micromachining (Gao et al., 1999).

In this dissertation, a non-local crystal plasticity model was developed that accounts for the non-local effect of geometrically necessary dislocations (GNDs) in governing the hardening response of polycrystalline FCC metals. The GNDs were characterized with two dislocation tensors: one a function of grain orientation $\left(\mathbf{R}_{\mathbf{h}}^{\mathbf{e}}\right)$ and one a function of the plastic deformation $\left(\mathbf{F}^{\mathbf{P}}\right)$. The two novel features associated with the non-local crystal plasticity model are the augmented kinematics (Polycrystal Kinematics) upon which the non-local model was built, and the non-local integral based approach used to estimate gradient quantities within the crystal plasticity model.

Experimental Hall-Petch results were used to validate the macro-scale simulation results of the non-local model, and experimental EBSD misorienation data was used to 
validate the micro-scale simulation results. Although this non-local model was used to study the Hall-Petch effect, it should be noted that this model could be used to predict any length scale dependent phenomena attributed to GNDs.

\subsection{Polycrystal Kinematics}

The conventional single crystal kinematics were augmented with a geometric argument to provide a rigorous and consistent method to initializing the GND state. The augmented kinematics (Polycrystal Kinematics) defined two dislocation tensors that together represent the GND state within a material. One of the dislocation tensors is a function of $\mathbf{R}_{\mathbf{h}}^{\mathbf{e}}$ and represents GNDs needed for compatibility in the reference configuration. In polycrystalline materials, this tensor represents the GND state in grain boundaries. The other dislocation tensor is a function of $\mathbf{F}^{\mathbf{P}}$ and represents GNDs accumulated during the deformation process. This dislocation tensor represents any evolving GND structures (like dislocation cells) in the material. An underlying assumption in this formulation is that any degree of incompatibility can be accommodated by GNDs. Such an assumption is clearly applicable to low angle boundaries but it is not clear that such an assumption is applicable to high angle boundaries.

The initial microstructure generated by the Polycrystal Kinematics was composed of interconnected regions of hardened grain boundary material surrounding softer grain interiors. The initial microstructure was an integral component of the Hall-Petch simulations. The simulated stress-strain curves from a non-local model that did not 
account for the initial microstructure were not stacked on top of each other as is seen experimentally. Instead, the model predicted curves with different slopes that pivot off the same point. On the other hand, the simulated stress-strain curves from a non-local model that did account for initial microstructure were stacked on top of each other, much more in-line with experimental observations. In addition, the non-local model without microstructure initialization predicted very little (2.3 MPa) difference in the yield strength for polycrystals with grain sizes of $14 \mu \mathrm{m}$ and $70 \mu \mathrm{m}$, while the non-local model with microstructure initialization predicted a significant difference in the yield strength (17.4 MPa) for the same polycrystals. Here again, the Hall-Petch results from the model with initialization compared qualitatively much better with experimental results.

In non-local models that do not account for the initial microstructure the material length scale effects evolve. There is no material length scale associated with the polycrystal prior to deformation meaning all length scale effects evolve from this point, which is why the stress-strain curves all pivot off the same point. As the non-local variable (usually plastic strain related) evolves, the model develops a material length scale that results in an increasingly stronger length scale dependent response. Because the length scale effects have not evolved enough at $0.2 \%$ strain, there is a small yield stress effect.

In non-local models that do account for initial microstructure there is an initial material length associated with the polycrystal prior to deformation: the width of the hardened grain boundary region. Since the width of similar grain boundary hardened regions is independent of grain size, a polycrystal with a smaller grain size produces a larger fraction of hardened material. Therefore, the smaller grain sizes have a higher 
volume fraction of hardened material resulting in a material with a higher yield strength. Thus the stress-strain curves are stacked on top of each other.

\subsection{Non-Local Integral Approach}

The gradient based dislocation tensors defined by polycrystal kinematics were numerically evaluated using a non-local integral based approach rather than the standard shape function based approach. The non-local integral based approach was based on the work of Gao and Huang (2001), but unlike the work of Gao and Huang, exponential damping functions were introduced in the non-local integral to describe the nature of the non-local interactions. The additional physics that the non-local integral approach brings to the model make this approach better suited to characterize microstructure.

A 1D numerical study investigated the properties of the non-local integral approach by comparing the gradient calculated via the non-local integral approach against known solutions. Both continuous and step functions were considered because the $\mathbf{F}^{\mathbf{P}}$ and $\mathbf{R}_{\mathrm{h}}^{\mathrm{e}}$ fields are continuous throughout the grain but they resemble a discontinuous step function at the grain boundary. For continuous functions, the error in the gradient approximated with non-local integral approach scales with the curvature of a continuous function: the more curvature, the greater the error. For discontinuous step functions, the non-local approach resolves the singularity at the grain boundary.

These gradient trends are very important because they translate directly to the $\rho^{\mathrm{GND}}$ and $\tau^{\mathrm{CSS}}$ profiles in the polycrystal. Since the spatial variations of $\mathbf{F}^{\mathbf{P}}$ and $\mathbf{R}_{\mathbf{h}}^{\mathbf{e}}$ inside the grain have little curvature, the $\rho^{\mathrm{GND}}$ and $\tau^{\mathrm{CSS}}$ profiles within the grain are unaffected 
by the non-local approach. At the grain boundaries, the $\rho^{\mathrm{GND}}$ and $\tau^{\mathrm{CSS}}$ profiles decay with a $1 / \mathrm{r}$ like dependence, similar to dislocation, similar to stress fields associated with dislocation-dislocation and dislocation-grain boundary interactions that determine hardening. This ability to define the $\rho^{\mathrm{GND}}$ and $\tau^{\mathrm{CSS}}$ profiles in the vicinity of grain boundaries is the primary advantage of the non-local integral based approach.

\subsection{Macroscale Hall-Petch Results}

The experimental results from Fernandes and Viera (2000) and Hansen (1983) were used to validate the simulation results of the non-local model. The tensile response of polycrystalline copper with grain sizes between $14 \mu \mathrm{m}$ and $240 \mu \mathrm{m}$ was simulated.

Qualitatively, the simulated data fit the experimental data reasonably well since the simulated data falls within the experimental scatter. The qualitative comparison between the best-fit Hall-Petch equations for the simulation and experimental results revealed that the simulation Hall-Petch dependence was closer to $\mathrm{d}^{-1}$ than $\mathrm{d}^{-0.5}$.

The influence of various simulation parameters were investigated to determine whether the $\mathrm{d}^{-1}$ dependence of yield strength was inherent to the model or caused by one or more of the simulation parameters.

- Grain shape. The yield strength for a polycrystal with square shaped grains was in general higher than that for a polycrystal composed of hex grains or realistic grain shapes. However, the Hall-Petch exponent for a polycrystal with square grains was lower than the other grain shapes. While grain shape does affect the 
Hall-Petch exponent, grain shape was not solely responsible for the $\mathrm{d}^{-1}$ dependence.

- Misorientation distribution. The effect of misorientation distribution was not strong and was not the cause the $\mathrm{d}^{-1}$ dependence. Even so, the closer the misorientation distribution was to the MacKenzie distribution (the theoretical misorientation distribution in random polycrystal), the lower the Hall-Petch exponent.

- Attenuation function. The Hall-Petch behavior predicted with a non-local model using an exponential attenuation function and a Gaussian attenuation function both predicted a d ${ }^{-1}$ like dependence.

- Boundary conditions. The yield strength was essentially unaffected by the boundary conditions. Boundary conditions did noticeably affect the material response starting around 5\% strain. However, the difference in the stress-strain curves using different boundary conditions was not great even at $17 \%$ strain. None of these parameters were solely responsible for the $\mathrm{d}^{-1}$ dependence of yield strength predicted by the non-local model.

The two non-local parameters $\omega$ and $\mathrm{C}$ displayed the greatest influence on the Hall-Petch exponent. The affect of $\omega$ and $\mathrm{C}$ was measured by considering the change in the grain boundary width and grain boundary height that resulted from changing $\omega$ and C. As $\mathrm{C}$ decreased, both the GB width and height decreased, while decreasing $\omega$ resulted in a larger GB width but smaller GB height. Correlating the changes in GB width and height with the resulting change in the Hall-Petch exponent revealed that the Hall-Petch exponent depended on GB height not GB width. Specifically, as the GB height got 
smaller, the Hall-Petch exponent got smaller. This result suggests that grain boundaries do not intensely harden the surrounding area.

The overall dependence of yield strength on grain size predicted by the non-local model was closer to $\mathrm{d}^{-1.0}$ than $\mathrm{d}^{-0.5}$. The $\mathrm{d}^{-1.0}$ dependence is similar to the dependence of yield strength on grain size predicted by Kock's composite model (Kocks, 1970). Despite this similarity, there are some fundamental differences differences between Kocks's composite model and the non-local model. The non-local model produces a graded, not step-function like, hardening profile near grain boundaries. The width of the grain boundary hardened region depends on the nature of the boundary itself (i.e. the misorientation between the two grains) and therefore does not need to be predefined. Finally, the width of the grain boundary hardened region can evolve with deformation if the grain boundary character changes. These differences illustrate the ways in which the non-local model accounts for more microscale detail than the Kocks's composite model and therefore is a superior modeling tool compared to Kock's composite model.

\subsection{Microscale Misorientation Results}

Experimental results for Brewer et al. (2006) were used to validate the microscale results predicted by the local and non-local crystal plasticity models. Brewer et al. (2006) tracked the change in grain orientations in polycrystalline nickel due to an applied tensile load with EBSD. The orientation maps collected at 1\%, 5\%, and $10 \%$ strain were then used to calculate the intragrain misorientation parameters LIMIS and AMIS. These experimental values were then compared with simulation LIMIS and AMIS results. 
Qualitatively, the LIMIS trends in the simulation results matched those observed experimentally. In both cases, LIMS was greatest at the grain boundary and it extends into the grain from these points at the grain boundaries. In fact, non-local model over states the effect at grain boundaries by predicting some amount of LIMIS at almost every grain boundary. Quantitatively, the simulatins underpredicted LIMIS. At 10\% strain the maximum experimental LIMIS was around $20^{\circ}$, while the maximum LIMIS in the nonlocal simulation was $7^{\circ}$.

The AMIS comparison between experiment and simulation was pretty good. The local model under predicted the experimental AMIS values indicating that the local model did not predict enough overall intragrain misorientation change within the material. The non-local model did a good job predicting the experimental AMIS values meaning that the non-local model did sufficiently predict the overall intragrain misorientation change within the material.

Considering both the LIMIS and the AMIS results together provides an overall picture of the microscale. The local model under predicts both LIMIS and AMIS. These results suggest that the local model predicts a spatial misorientation distribution that is too uniform (LIMIS) and on average there is not enough intragrain misorientation (AMIS). The non-local model, on the other hand, under predicts the LIMIS but did correctly predict AMIS. In this case, the non-local model predicted a spatial misorientation distribution that was too uniform, but on average had the correct amount of misorientation. These microscale results illustrate that neither the local nor the nonlocal crystal plasticity models correctly accounts for all the microscale physics. 
However, these results also demonstrate that the non-local model is an improvement over the local model. 


\section{APPENDIX A}

\section{SYMMETRY OPERATORS FOR CUBIC SYMMETRY}

The symmetry operators for the rotation matrix $\left(\begin{array}{lll}\text { a11 } & \text { a12 } & \text { a13 } \\ \text { a21 } & \text { a22 } & \text { a23 } \\ \text { a31 } & \text { a32 } & \text { a33 }\end{array}\right)$

$1\left(\begin{array}{lll}\mathrm{a} 11 & \mathrm{a} 12 & \mathrm{a} 13 \\ \mathrm{a} 21 & \mathrm{a} 22 & \mathrm{a} 23 \\ \mathrm{a} 31 & \mathrm{a} 32 & \mathrm{a} 33\end{array}\right)$

$7\left(\begin{array}{ccc}-\mathrm{a} 21 & -\mathrm{a} 22 & -\mathrm{a} 23 \\ -\mathrm{a} 31 & -\mathrm{a} 32 & -\mathrm{a} 33 \\ \mathrm{a} 11 & \mathrm{a} 12 & \mathrm{a} 13\end{array}\right)$

$13\left(\begin{array}{lll}-\mathrm{a} 31 & -\mathrm{a} 32 & -\mathrm{a} 33 \\ -\mathrm{a} 21 & -\mathrm{a} 22 & -\mathrm{a} 23 \\ -\mathrm{a} 11 & -\mathrm{a} 12 & -\mathrm{a} 13\end{array}\right)$

$2\left(\begin{array}{ccc}-\mathrm{a} 11 & -\mathrm{a} 12 & -\mathrm{a} 13 \\ \mathrm{a} 21 & \mathrm{a} 22 & \mathrm{a} 23 \\ -\mathrm{a} 31 & -\mathrm{a} 32 & -\mathrm{a} 33\end{array}\right)$

$8\left(\begin{array}{ccc}a 21 & a 22 & a 23 \\ -a 31 & -a 32 & -a 33 \\ -a 11 & -a 12 & -a 13\end{array}\right)$

$14\left(\begin{array}{ccc}a 31 & \mathrm{a} 32 & \mathrm{a} 33 \\ -\mathrm{a} 21 & -\mathrm{a} 22 & -\mathrm{a} 23 \\ \mathrm{a} 11 & \mathrm{a} 12 & \mathrm{a} 13\end{array}\right)$

$3\left(\begin{array}{ccc}-a 11 & -a 12 & -a 13 \\ -a 21 & -a 22 & -a 23 \\ a 31 & a 32 & a 33\end{array}\right)$

$9\left(\begin{array}{lll}a 31 & \text { a32 } & \text { a33 } \\ \text { a11 } & \text { a12 } & \text { a13 } \\ \text { a21 } & \text { a22 } & \text { a23 }\end{array}\right)$

$15\left(\begin{array}{ccc}a 31 & \text { a32 } & \text { a33 } \\ \mathrm{a} 21 & \mathrm{a} 22 & \mathrm{a} 23 \\ -\mathrm{a} 11 & -\mathrm{a} 12 & -\mathrm{a} 13\end{array}\right)$

$4\left(\begin{array}{ccc}a 11 & a 12 & a 13 \\ -a 21 & -a 22 & -a 23 \\ -a 31 & -a 32 & -a 33\end{array}\right)$

$10\left(\begin{array}{ccc}-a 31 & -a 32 & -a 33 \\ a 11 & a 12 & a 13 \\ -a 21 & -a 22 & -a 23\end{array}\right)$

$16\left(\begin{array}{ccc}-a 31 & -a 32 & -a 33 \\ a 21 & a 22 & a 23 \\ a 11 & a 12 & a 13\end{array}\right)$

$5\left(\begin{array}{lll}a 21 & \text { a22 } & \text { a23 } \\ \text { a31 } & \text { a32 } & \text { a33 } \\ \text { a11 } & \text { a12 } & \text { a13 }\end{array}\right)$

$11\left(\begin{array}{ccc}-\mathrm{a} 31 & -\mathrm{a} 32 & -\mathrm{a} 33 \\ -\mathrm{a} 11 & -\mathrm{a} 12 & -\mathrm{a} 13 \\ \mathrm{a} 21 & \mathrm{a} 22 & \mathrm{a} 23\end{array}\right)$

$17\left(\begin{array}{lll}-\mathrm{a} 21 & -\mathrm{a} 22 & -\mathrm{a} 23 \\ -\mathrm{a} 11 & -\mathrm{a} 12 & -\mathrm{a} 13 \\ -\mathrm{a} 31 & -\mathrm{a} 32 & -\mathrm{a} 33\end{array}\right)$

$6\left(\begin{array}{ccc}-a 21 & -a 22 & -a 23 \\ \text { a31 } & \text { a32 } & \text { a33 } \\ -a 11 & -a 12 & -a 13\end{array}\right)$

$12\left(\begin{array}{ccc}a 31 & \mathrm{a} 32 & \mathrm{a} 33 \\ -\mathrm{a} 11 & -\mathrm{a} 12 & -\mathrm{a} 13 \\ -\mathrm{a} 21 & -\mathrm{a} 22 & -\mathrm{a} 23\end{array}\right)$

$18\left(\begin{array}{ccc}a 21 & \text { a22 } & \text { a23 } \\ -\mathrm{a} 11 & -\mathrm{a} 12 & -\mathrm{a} 13 \\ \mathrm{a} 31 & \mathrm{a} 32 & \mathrm{a} 33\end{array}\right)$ 
$19\left(\begin{array}{ccc}a 21 & a 22 & a 23 \\ a 11 & a 12 & a 13 \\ -a 31 & -a 32 & -a 33\end{array}\right)$

$20\left(\begin{array}{ccc}-\mathrm{a} 21 & -\mathrm{a} 22 & -\mathrm{a} 23 \\ \mathrm{a} 11 & \mathrm{a} 12 & \mathrm{a} 13 \\ \mathrm{a} 31 & \mathrm{a} 32 & \mathrm{a} 33\end{array}\right)$

$21\left(\begin{array}{ccc}-a 11 & -a 12 & -a 13 \\ -a 31 & -a 32 & -a 33 \\ -a 21 & -a 22 & -a 23\end{array}\right)$

$22\left(\begin{array}{ccc}a 11 & \text { a12 } & \text { a13 } \\ -a 31 & -a 32 & -a 33 \\ a 21 & a 22 & a 23\end{array}\right)$

$23\left(\begin{array}{ccc}\mathrm{a} 11 & \mathrm{a} 12 & \mathrm{a} 13 \\ \mathrm{a} 31 & \mathrm{a} 32 & \mathrm{a} 33 \\ -\mathrm{a} 21 & -\mathrm{a} 22 & -\mathrm{a} 23\end{array}\right)$

$24\left(\begin{array}{ccc}-a 11 & -a 12 & -a 13 \\ \text { a31 } & \text { a32 } & \text { a33 } \\ \text { a21 } & \text { a22 } & \text { a23 }\end{array}\right)$ 


\section{APPENDIX B \\ KOCK'S COMPOSITE MODEL FOR POLYCRYSTALLINE MATERIALS}

Kocks (1970) derived an analytical solution for the dependence of grain size (d) on the yield strength for a polycrystal containing square grains. Each of these square grains was modeled as a two-component material. The first component was a soft grain interior that had an area $\mathrm{A}_{\mathrm{I}}$ and a yield strength $\sigma_{\mathrm{I}}$. The other component was a hardened grain boundary region that had an area $\mathrm{A}_{\mathrm{GB}}$ and a yield strength $\sigma_{\mathrm{GB}}$. The hardened grain boundary region had a constant thickness $\mathrm{w}_{\mathrm{GB}}$ that was independent of grain size. For simplicity, only 1 grain from the model polycrystal proposed by Kocks is shown in Figure B.1.
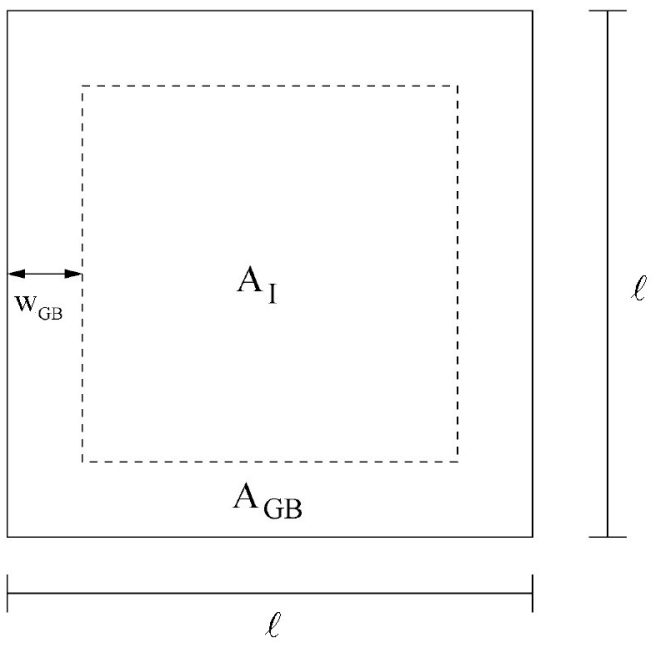

\begin{tabular}{|c|c|}
\hline & Area \\
\hline Grain & $\mathrm{A}_{\mathrm{T}}=\mathrm{d}^{2}$ \\
Grain Interior & $\mathrm{A}_{\mathrm{I}}=\left(\mathrm{d}-2 \mathrm{w}_{\mathrm{GB}}\right)^{2}$ \\
Grain Boundary & $\mathrm{A}_{\mathrm{GB}}=4 \mathrm{w}_{\mathrm{GB}}{ }^{2}+4 \mathrm{w}_{\mathrm{GB}} \mathrm{d}$ \\
\hline
\end{tabular}

Grain Size : $d=\sqrt{\mathrm{A}_{\mathrm{T}}}=\ell$

Figure B.1 - A square grain composed of a hardened grain boundary region, $\mathrm{A}_{\mathrm{GB}}$, and softer grain core, $A_{I}$.

Kocks proposed that the yield strength of the polycrystal, $\sigma_{\mathrm{ys}}$, was the Voight average of $\sigma_{\mathrm{I}}$ and $\sigma_{\mathrm{GB}}$ 


$$
\sigma_{\mathrm{ys}}=\sigma_{\mathrm{I}} \frac{\mathrm{A}_{\mathrm{I}}}{\mathrm{A}_{\mathrm{T}}}+\sigma_{\mathrm{GB}} \frac{\mathrm{A}_{\mathrm{GB}}}{\mathrm{A}_{\mathrm{T}}} .
$$

If $\ell>>\mathrm{w}_{\mathrm{GB}}, \mathrm{A}_{\mathrm{I}}$ simplifies to $\mathrm{d}^{2}-4 \mathrm{w}_{\mathrm{GB}} \mathrm{d}$ and $\mathrm{A}_{\mathrm{GB}}$ to $4 \mathrm{w}_{\mathrm{GB}} \mathrm{d}$. With these values for $\mathrm{A}_{\mathrm{I}}$ and $\mathrm{A}_{\mathrm{GB}}$, Equation $\mathrm{B} 1$ becomes

$$
\sigma_{\mathrm{ys}}=\sigma_{\mathrm{I}} \frac{\ell^{2}-4 \mathrm{w}_{\mathrm{GB}} \mathrm{d}}{\ell^{2}}+\sigma_{\mathrm{GB}} \frac{4 \mathrm{w}_{\mathrm{GB}} \ell}{\ell^{2}}=\sigma_{\mathrm{I}}\left(1-\frac{4 \mathrm{w}_{\mathrm{GB}}}{\ell}\right)+\sigma_{\mathrm{GB}} \frac{4 \mathrm{w}_{\mathrm{GB}}}{\ell} .
$$

By rearranging terms and substituting $d=\ell$, an expression that clearly shows a $\mathrm{d}^{-1}$ dependence can be derived

$$
\sigma_{y \mathrm{~s}}=\sigma_{\mathrm{I}}+\frac{4 \sigma_{\mathrm{I}} \mathrm{w}_{\mathrm{GB}}}{\mathrm{d}}\left(\frac{\sigma_{\mathrm{GB}}}{\sigma_{\mathrm{I}}}-1\right) .
$$

Kocks's analysis can be applied to any polycrystal with grains that have the same shape. For example, a polycrystal with hexagon shaped grains.

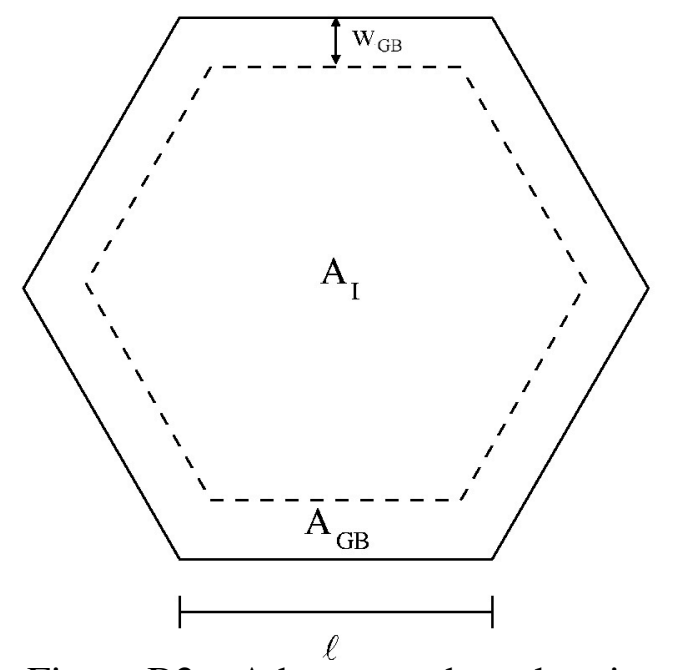

\begin{tabular}{|c|c|}
\hline & Area \\
\hline Grain & $\mathrm{A}_{\mathrm{T}}=\mathrm{C}_{\mathrm{Hex}} \mathrm{d}^{2}$ \\
Grain Interior & $\mathrm{A}_{\mathrm{I}}=\mathrm{C}_{\mathrm{Hex}}\left(\mathrm{d}-\mathrm{w}_{\mathrm{GB}}\right)^{2}$ \\
Grain Boundary & $\mathrm{A}_{\mathrm{I}}=\mathrm{C}_{\mathrm{Hex}}\left(2 \mathrm{w}_{\mathrm{GB}} \mathrm{d}-\mathrm{x}^{2}\right)$ \\
\hline
\end{tabular}

$$
\text { where } \mathrm{C}_{\mathrm{Hex}}=\frac{3 \sqrt{3}}{2}
$$

Grain Size $: \mathrm{d}=\sqrt{\mathrm{A}_{\mathrm{T}}}=\sqrt{\mathrm{C}_{\mathrm{Hex}}} \ell$

Figure B2 - A hexagon shaped grain composed of a hardened grain boundary region, $\mathrm{A}_{\mathrm{GB}}$, and softer grain core, $\mathrm{A}_{\mathrm{I}}$.

If it is assumed that the yield strength is the Voight average of $\sigma_{\mathrm{I}}$ and $\sigma_{\mathrm{GB}}$ and that $\ell$ $>>\mathrm{w}_{\mathrm{GB}}$, then the polycrystal's yield strength becomes 


$$
\begin{aligned}
\sigma_{\mathrm{ys}} & =\sigma_{\mathrm{I}} \mathrm{C}_{\mathrm{Hex}}\left(\frac{\ell^{2}-2 \mathrm{w}_{\mathrm{GB}} \ell}{\ell^{2}}\right)+\sigma_{\mathrm{GB}} \mathrm{C}_{\mathrm{Hex}}\left(\frac{2 \mathrm{w}_{\mathrm{GB}} \ell}{\ell^{2}}\right) \\
& =\sigma_{\mathrm{I}} \mathrm{C}_{\mathrm{Hex}}\left(1-\frac{2 \mathrm{w}_{\mathrm{GB}}}{\ell}\right)+\sigma_{\mathrm{GB}} \mathrm{C}_{\mathrm{Hex}}\left(\frac{2 \mathrm{w}_{\mathrm{GB}}}{\ell}\right)
\end{aligned}
$$

By rearranging some terms and substituting $\mathrm{d}=\sqrt{\mathrm{C}_{\mathrm{Hex}}} \ell$, an expression that clearly shows $\mathrm{a} \mathrm{d}^{-1}$ dependence is derived

$$
\sigma_{\mathrm{ys}}=\mathrm{C}_{\mathrm{Hex}} \sigma_{\mathrm{I}}+\frac{2 \mathrm{C}_{\mathrm{Hex}} \sqrt{\mathrm{C}_{\mathrm{Hex}}} \sigma_{\mathrm{I}} \mathrm{w}_{\mathrm{GB}}}{\mathrm{d}}\left(\frac{\sigma_{\mathrm{GB}}}{\sigma_{\mathrm{I}}}-1\right) .
$$




\section{APPENDIX C}

\section{EXPERIMENTAL HALL-PETCH DATA}

The effect of grain size on the yield strength of copper is well documented in the literature. With some effort different experimental data sets containing Hall-Petch data were found: 1) Carreker and Hibbard (1953), 2) Feltham and Meakin (1957), 3) Johnston and Feltner (1970), 4) Thompson and Baskes (1973), and 5) Hansen (1983). The reported best-fit Hall-Petch equations from each data set have been plotted in Figure C.1. In Figure C.1, the average of the 5 experiments has also been plotted.

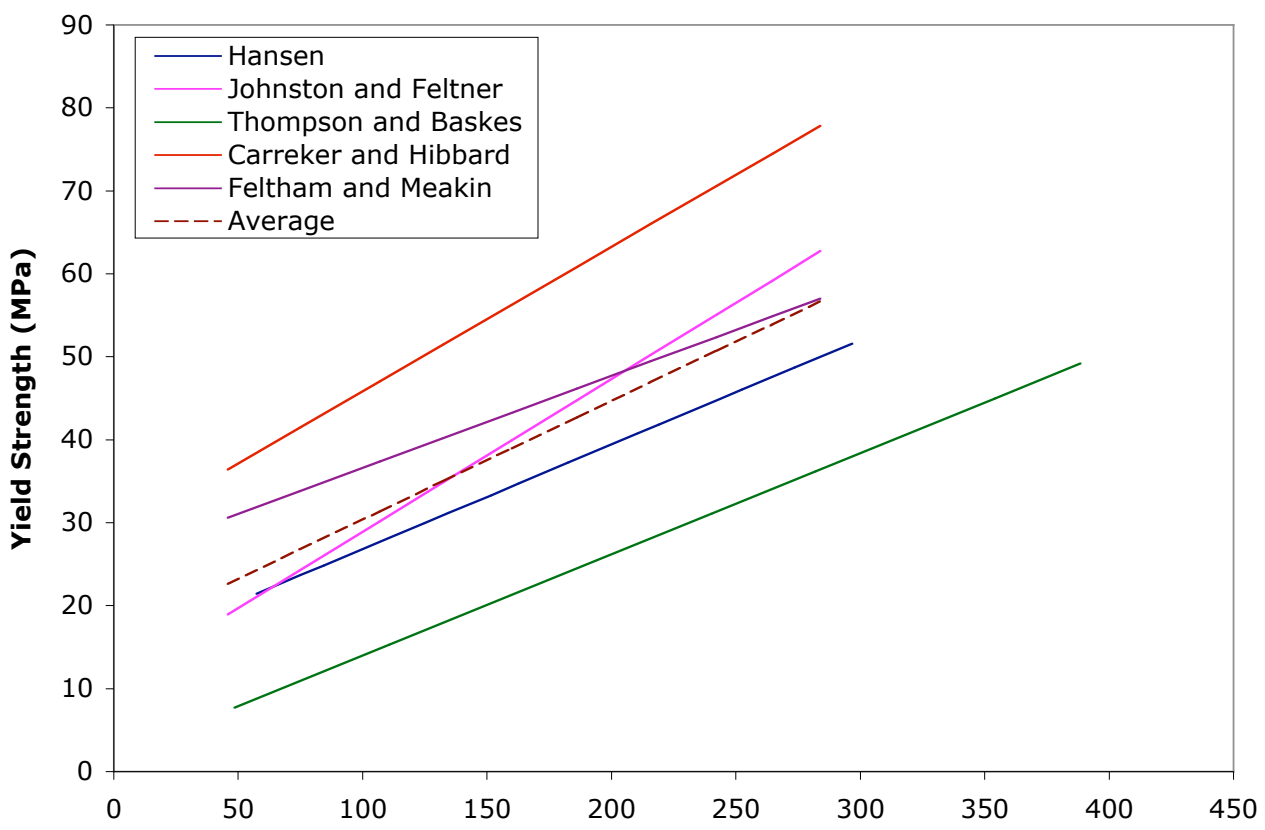

Figure C.1 -Experimental Hall-Petch data for copper

The equations plotted in Figure C.1 are tabulated in Table C.1. 
Table C.1 -Best-fit Hall-Petch equations to experimental Hall-Petch data for copper

\begin{tabular}{|c|c|}
\hline & $\begin{array}{c}\text { Hall-Petch Equation } \\
\left(\sigma_{\mathrm{y}}=\sigma_{0}+\mathrm{k} \mathrm{d}^{-\mathrm{n}}\right)\end{array}$ \\
\hline Hansen & $\sigma_{\mathrm{y}}=14.216+0.126 \mathrm{~d}^{-0.50}$ \\
\hline Johnston and Feltner & $\sigma_{\mathrm{y}}=10.494+0.184 \mathrm{~d}^{-0.50}$ \\
\hline Thompson and Baskes & $\sigma_{\mathrm{y}}=1.793+0.122 \mathrm{~d}^{-0.52}$ \\
\hline Carreker and Hibbard & $\sigma_{\mathrm{y}}=28.439+0.173 \mathrm{~d}^{-0.50}$ \\
\hline Feltham and Meakin & $\sigma_{\mathrm{y}}=25.497+0.112 \mathrm{~d}^{-0.50}$ \\
\hline & \\
\hline Average & $\sigma_{\mathrm{y}}=16.088+0.143 \mathrm{~d}^{-0.50}$ \\
\hline
\end{tabular}

The average value in Table C. 1 was used as a metric with which to compare simulation results and trends.

At first glance, it would appear that there is a plethora of available experimental data that could be used to verify simulation results. However, modeling the Hall-Petch effect requires a data set with multiple stress-strain curves at different grain sizes in order to fit the various model parameters. From the 5 aforementioned data sets, only Carreker and Hibbard (1953), and Thompson and Baskes (1973) included stress-strain curves in their publication. The quality of the Carreker and Hibbard (1953) reprint made was not good enough to extract the stress-strain curves. The stress-strain curves from Thompson and Baskes (1973) exhibited curve-crossing ${ }^{11}$ and therefore avoided. This lack of modeling friendly experimental data sets in the literature makes model verification difficult.

In this research project, two experimental data sets were used to verify the nonlocal model: Hansen (1983) and Fernandes and Viera (2000). After a private

${ }^{11}$ Curve crossing happens when a specimen with a small grain size has a higher flow stress at low strains compared to a larger grained specimen, but at larger strains this ratio is reversed. Curve crossing is due to the formation of strong textures in the large grained sample (Hansen, 1983). 
communication, Hansen provided "representative" stress-strain curves from a previous publication (Hansen, 1979). It was not clear from the private communication whether these stress-strain curves were a part of the Hall-Petch data reported in the latter publication Hansen (1983). With these "representative" stress-strain curves, the Hansen data set could be modeled. The Fernandes and Viera (2000) experimental data was used because it contained 5 stress-strain curves at different grain sizes. In this case, $\sigma_{\mathrm{ys}}$ was estimated from the stress-strain curves. So in one case, the exact Hall-Petch data is available with an estimate on the stress-strain curves; in the other case, the exact stressstrain curves are available with an estimate on the Hall-Petch data. 


\section{APPENDIX D}

\section{THE LOCAL INTRAGRAIN MISORIENTATION (LIMIS) AND AVERAGE MISORIENTATION (AMIS) CALCULATIONS}

\section{D.1 LIMIS}

The LIMIS calculation was done in two steps. The first step calculated the average misorientation between an element and its neighbors. For example, consider the square shaped grain with 9 elements shown in Figure D.1.

\begin{tabular}{|c|c|c|}
\hline 1 & 2 & 3 \\
\hline 4 & 5 & 6 \\
\hline 7 & 8 & 9 \\
\hline
\end{tabular}

Figure D.1 - Square shaped composed of 9 elements.

The average misorientation at element $5\left(\bar{\theta}^{5}\right)$ was the average of the misorientations between element 5 and its eight neighbors

$$
\bar{\theta}^{5}=\left(\theta^{5-1}+\theta^{5-2}+\theta^{5-3}+\theta^{5-4}+\theta^{5-6}+\theta^{5-7}+\theta^{5-8}+\theta^{5-9}\right) / 8
$$

where $\theta^{\mathrm{a}-\mathrm{b}}$ is the misorientation at element a between elements $\mathrm{a}$ and $\mathrm{b}$. In this calculation, $\theta^{\mathrm{a}-\mathrm{b}}$ was calculated in the standard manner: using the orientation tensors for elements $\mathrm{a}$ and $\mathrm{b}$. The LIMIS calculation did not consider misorientations across grain boundaries. Thus, $\bar{\theta}^{1}$ was the average of the misorientations between element 1 and the three neighbors (elements 2, 4, and 5) contained within the same grain. Once the average 
misorientation at each element had been calculated, the element with the lowest average misorientation was found. For notation purposes, the element with lowest average misorientation is element $*$.

In the second step, the local intragrain misorientation at each element was calculated. The LIMIS calculation was similar to the previously described average misorientation calculation. In the LIMIS calculation, the misorientation between two elements was calculated using orientation corresponding to the element with the lowest misorientation value rather than the orientation of the element itself. So the LIMIS calculation used $\theta^{*-\mathrm{b}}$ rather than $\theta^{\mathrm{a}-\mathrm{b}}$. For example, LIMUS at element 5 was calculated via

$$
\mathrm{LIMIS}=\left(\theta^{*-1}+\theta^{*-2}+\theta^{*-3}+\theta^{*-4}+\theta^{*-6}+\theta^{*-7}+\theta^{*-8}+\theta^{*-9}\right) / 8 .
$$

\section{D.2 AMIS}

A grain AMIS value is calculated as the average misorientation between all the elements with each grain. For the 9 element grain in Figure D.1, the grain AMIS would be calculated as

$$
\text { grain AMIS }=\left(\begin{array}{l}
\theta^{1-2}+\theta^{1-3}+\theta^{1-4}+\theta^{1-5}+\theta^{1-6}+\theta^{1-7}+\theta^{1-8}+\theta^{1-9}+ \\
\theta^{2-3}+\theta^{2-4}+\theta^{2-5}+\theta^{2-6}+\theta^{2-7}+\theta^{2-8}+\theta^{2-9}+ \\
\theta^{3-4}+\theta^{3-5}+\theta^{3-6}+\theta^{3-7}+\theta^{3-8}+\theta^{3-9}+ \\
\theta^{4-5}+\theta^{4-6}+\theta^{4-7}+\theta^{4-8}+\theta^{4-9}+ \\
\theta^{5-6}+\theta^{5-7}+\theta^{5-8}+\theta^{5-9}+ \\
\theta^{6-7}+\theta^{6-8}+\theta^{6-9}+ \\
\theta^{7-8}+\theta^{7-9}+ \\
\theta^{8-9}
\end{array}\right) / 36
$$


The grain AMIS calculation leaves out redundant calculations making it computationally efficient. For example, the term $\theta^{2-1}$ is not calculated in line 2 of the grain AMIS equation because it is identical to $\theta^{1-2}$, which has already been calculated. This is the reason each line becomes 1 term shorter. The AMIS value for the material is the average of the grain AMIS values. 


\section{REFERENCES}

Acharya, A., and Bassani, J.L. (2000) "Lattice incompatibility and a gradient theory of crystal plasticity," Journal of the Mechanics and Physics of Solids, Vol. 48, 1565-1595.

Acharya, A., and Beaudoin, A.J. (2000) "Grain-size effects in viscoplastic polycrystals at moderate strains," Journal of the Mechanics and Physics of Solids, Vol. 48, 2213-2230.

Acharya, A., and Shawki, T.G. (1994) "Thermodynamic restrictions on constitutive equations for second deformation gradient inelastic behavior," Journal of the Mechanics and Physics of Solids, Vol. 43, 1751-1772.

Aifantis, E.C. (1984) "On the microstructural origin of certain inelastic models," Journal of Engineering Materials and Technology, Vol. 106, 326-330.

Anand, L. and Kothari, M. (1996) "Computational procedure for rate-independent crystal plasticity," Journal of the Mechanics and Physics of Solids, Vol. 44, 525-558.

Ananthakrishna, G. (1993) "Formation, propagation of bands and chaos in jerky flow," Scripta Metallurgica et Materialia, Vol. 1993, 1183-1188.

Armstrong, R.W., Codd, I., Douthwaite, R.M., Petch, N.J. (1962) “The plastic deformation of polycrystalline aggregates," Philosophical Magazine, Vol. 7, 45-58.

Arsenlis, A. and Parks, D.M. (1999) "Crystallographic aspects of geometricallynecessary and statistically-stored dislocation density," Acta Materialia, Vol. 47, 15971611.

Asaro, R.J. and Rice, J.R. (1977) "Strain localization in ductile single crystals,"," Journal of the Mechanics and Physics of Solids, Vol. 25, 309-338.

Asaro, R.J. (1983) “Crystal Plasticity,” Journal of Applied Mechanics, Vol. 50, 921-934.

Ashby, M.F. (1970) "The deformation of plastically non-homogeneous materials," Philosophical Magazine, Vol. 21, 399-424.

Baldwin, W.M. (1958) "Yield strength of metals as a function of grain size," Acta Metallurgica, Vol. 6, 139-141.

Bammann, D.J. (2001) "A model of crystal plasticity containing a natural length scale," Materials Science and Engineering A, Vol. 309-310, 406-410.

Bammann, D.J. and Aifantis, E.C. (1982) "On a Proposal for a Continuum with Microstructure,” Acta Mechanica, Vol. 45, 91-121. 
Barabash, R.I., Ice, G.E., Pang, J.W.L. (2005) "Gradients of geometrically necessary dislocations from white beam microdiffraction," Materials Science and Engineering A, Vol. 400-401, 125-131.

Basinski, S.F., and Basinski, Z.S. (1966) Recrystallization, Grain Growth, and Textures, American Society of Metals, Metals Park, OH.

Bassani, J.L. (2001) "Incompatibility and a simple gradient theory of plasticity," Journal of the Mechanics and Physics of Solids, Vol. 49, 1983-1996.

Bassani, J.L. and Wu, T.Y. (1991) "Latent hardening in single crystals II. Analytical characterization and predictions," Proceedings of the Royal Society of London Series AMathematical and Physical Sciences, Vol. 435, 21-41.

Bazant, Z.P. and Lin, F.B. (1988) "Non-local yield limit degradation," International Journal for Numerical Methods in Engineering, Vol. 26, 1805-1823.

Bazant, Z.P. and Ozbolt, J. (1990) "Nonlocal microplane model for fracture, damage, and size effect in structures," Journal of Engineering Mechanics, Vol. 116, 2485-2505

Bazant, Z.P. and Jirasek, M. (2002) "Nonlocal integral formulations of plasticity and damage: survey of progress," Journal of Engineering Mechanics, Vol. 128, 1119-1149.

Beaudoin, A.J., Acharya, A., Chen, S.R., Korzekwa, D.A., Stout, M.G. (2000)

"Consideration of grain size effect and kinetics in the plastic deformation of metal polycrystals," Acta Materialia, Vol. 48, 3409-3423.

Biffle, J.H., 1987. A three-dimensional finite element computer program for the nonlinear quasi-static response of solids with the conjugate gradient method. SAND87-1305. Sandia National Laboratories, Albuquerque, NM.

Bilby, B.A., Bullough, R., Smith, E., (1955) "Continuous distributions of dislocations: a new application of methods of non-riemannian geometry," Proceedings of the Royal Society of London Series A - Mathematical and Physical Sciences, Vol. 231, 263-273.

Borino, G. and Polizzotto, C. (1999) "Comments on 'Nonlocal strain softening bar revisited' by Christer Nilsson", International Journal of Solids and Structures, Vol. 36, 3085-3091.

Brenner, S.S. (1956) “Tensile strength of whiskers," Journal of Applied Physics, Vol. 27, 1484-1491.

Brewer, L.N., Counts, W.A., Buchheit, T.E., Battaile, C.C., Michael, J.R. (2006) "Comparison of experimental determination and simulation of polycrystalline plasticity in nickel," To be published. 
Carreker, R.P. and Hibbard, W.R. (1953) "Tensile deformation of high-purity copper as a function of temperature, strain rate, and grain size," Acta Metallurgica, Vol. 1, 656-663.

Carreker, R.P. (1957) "Tensile deformation of silver as a function of temperature, strain rate, and grain size," Transactions of the American Institute of Mining and Metallurgical Engineers, Vol. 209, 112-115.

Carreker, R.P. and Hibbard, W.R. (1957) "Tensile deformation of aluminum as a function of temperature, strain rate, and grain size," Transactions of the American Institute of Mining and Metallurgical Engineers, Vol. 209, 1157-1163.

Cermelli, P. and Gurtin, M.E. (2001) "On the characterization of geometrically necessary dislocations in finite plasticity," Journal of Mechanics and Physics of Solids, Vol. 49, 1539-1568.

Cheng, S., Spencer, J.A., Milligan, W.W. (2003) "Strength and tension/compression asymmetry in nanostructured and ultrafine-grain metals," Acta Materialia, Vol. 51, 45054518.

Clayton, J.D. and McDowell, D.L. (2003a) “A multiscale multiplicative decomposition for elastoplasticity of polycrystals," International Journal of Plasticity, Vol. 19, 14011444.

Clayton, J.D. and McDowell, D.L. (2003b) "Finite polycrystalline elastoplasticity and damage: multiscale kinematics," International Journal of Solids and Structures, Vol. 40, 5669-5688.

Coleman, B.D. and Noll, W. (1963) "The thermodynamics of elastic materials with heat conduction and viscocity," Archive for Rational Mechanics and Analysis, Vol. 13, 167178.

Coleman, B.D. and Gurtin, M.E. (1967) “Thermodynamics with internal state variables." Journal of Chemical Physics, Vol. 47, 597-613.

Cosserat, E. and Cosserat, F. (1909) Theorie des corps defomables, Herrman, Paris.

Cotrell, A.H. (1958) "Theory of brittle fracture in steel and similar metals," Transactions of the Metallurgical Society of AIME, Vol. 212, 192-203.

Cuitino, A.M. and Ortiz, M. (1992) "Computational modeling of single crystals" Modelling and Simulation in Matierals Science and Engineering, Vol. 1, 225-263.

Dillion, O.W. and Kratochvil, J. (1970) "A strain gradient theory of plasticity," International Journal of Solids and Structures, Vol. 6, 1513-1533. 
Duan, D. M., Wu, N.Q., Slaugher, W.S., Mao, S.X. (2001) "Length scale effect on mechanical behavior due to strain gradient plasticity," Materials Science and Engineering A, Vol. 303, 241-249.

Edelen, D.G.B. (1969) "Protoelastic bodies with large deformations," Archive for Rational Mechanics and Analysis, Vol. 34, 283-300.

Edelen, D.G.B., Green, A.E., Laws, N. (1971) "Nonlocal continuum mechanics," Archive for Rational Mechanics and Analysis, Vol. 43, 36-44.

Eringen, A.C. (1966) "A unified theory of thermomechanical materials," International Journal of Engineering Science, Vol. 4, 179-202.

Eringen, A.C. (1972) "Linear theory of nonlocal elasticity and dispersion of plane waves," International Journal of Engineering Science, Vol. 10, 425-435.

Eringen, A.C. and Edelen, D.G.B. (1972) “On nonlocal elasticity,” International Journal of Engineering Science, Vol. 10, 233-248.

Eringen, A.C. and Kim, B.S. (1974) "Stress concentration at the tip of a crack," Mechanical Research Communications, Vol. 1, 233-237.

Eringen, A.C., Speziale, C.G., Kim, B.S. (1977) "Crack-tip problem in nonlocal elastcitiy," Journal of the Mechanics and Physics of Solids, Vol. 25, 339-355.

Eringen, A.C. (1981) “On nonlocal plasticity” International Journal of Engineering Science, Vol. 19, 1461-1474.

Eringen, A.C. (1983) "Theories of nonlocal plasticity" International Journal of Engineering Science, Vol. 21, 741-751.

Espinosa, H.D., Prorok, B.C., Peng, B. (2004) "Plasticity size effects in free-standing submicron polycrystalline fcc films subjected to pure tension," Journal of the Mechanics and Physics of Solids, Vol. 52, 667-689.

Estrin, Y. and Mecking, H. (1983) "A unified phenomenological description of work hardening and creep based on one-parameter models," Acta Metallurgica, Vol.32, 57-70.

Evers, L.P., Brekelmans, W.A.M., Geers, M.G.D. (2004a) "Non-local crystal plasticity model with intrinsic SSD and GND effects," Journal of the Mechanics and Physics of Solids, Vol. 52, 2379-2401.

Evers, L.P., Brekelmans, W.A.M., Geers, M.G.D. (2004b) "Scale dependent crystal plasticity framework with dislocation density and grain boundary effects," International Journal of Solids and Structures, Vol. 41, 5209-5230. 
Feltham, P. and Meakin, J.D. (1957) "On the mechanism of workhardening in facecentered cubic metals, with special reference to polycrystalline copper," Philosophical Magazine, Vol. 2, 105-112.

Fernandes, J.V. and Vieira, M.F. (2000) "Further development of the hybrid model for polycrystal deformation," Acta Materialia, Vol. 48, 1919-1930.

Flanagan, D.P. and Belytschko, T. (1981) "A uniform strain hexahedron and quadrilateral with orthogonal hourglass control," International Journal for Numerical Methods in Engineering, Vol. 17, 597-609.

Fleck, N.A. and Hutchinson, J.W. (1993) "A phenomenological theory for strain gradient effects in plasticity," Journal of the Mechanics and Physics of Solids, Vol. 41, 18251857.

Fleck, N.A. and Hutchinson, J.W. (1997) "Strain gradient plasticity," Advances in Applied Mechanics, Eds: J.W. Hutchinson and T.Y. Wu, Vol. 33, 295-361.

Fleck, N.A. and Hutchinson, J.W. (2001) "A reformulation of strain gradient plasticity," Journal of the Mechanics and Physics of Solids, Vol. 49, 2245-2271.

Fleck, N.A., Muller, G.M., Ashby, M.F., Hutchinson, J.W. (1994) "Strain gradient plasticity: theory and experiment," Acta Metallurigca et Materialia, Vol. 42, 475-487.

Follstaedt, D.M., Knapp, J.A., Myers, S.M. (2003) "Strengthening by High Densities of Nanometer-Size Precipitates: Oxides in Ni." Metallurgical and Materials Transactions A, Vol. 34A, 935-949.

Fu, H.-H., Benson, J., Meyers, M.A. (2001) "Analytical and computational description of effect of grain size on yield stress of metals," Acta Materialia, Vol. 49, 2567-2582

Fukuoka, C., Morishima, K., Yoshizawa, H., Mino, K. (2005) "Misorientation development in grains of tensile strained and crept 2.25\%Cr-1\%Mo steel," Scripta Materialia, Vol. 46, 61-66.

Gane, N. and Cox, J.M. (1970) "The Micro-hardness of metals at very low loads," Philosophical Magazine, Vol. 22, 881-891.

Gao, H., Huang, Y., Nix, W.D., Hutchinson, J.W. (1999) "Mechanism-based strain gradient plasticity-I. Theory," Journal of the Mechanics and Physics of Solids, Vol. 47, 1239-1263.

Gao, H., Huang, Y., Nix, W.D., Hutchinson, J.W. (1999) "Mechanism-based strain gradient plasticity-II. Analysis," Journal of the Mechanics and Physics of Solids, Vol. 48, 99-128. 
Gao, H., and Huang, Y. (2001) "Taylor-based nonlocal theory of plasticity," International Journal of Solids and Structures, Vol. 38, 2615-2637.

Glazov, M.V., and Laird, C. (1995) "Size effects of dislocation patterning in fatigued metals," Acta Metallurigca et Materialia, Vol. 43, 2849-2857.

Green, A.E. and Rivlin, R.S. (1964) "Simple force and stress multipoles," Archive for Rational Mechanics and Analysis, Vol. 16, 325-353.

Greer, J.R., Oliver, W.C., Nix, W.D. (2005) "Size dependence of mechanical properties of gold at the micron scale in the absence of strain gradients," Acta Materialia, Vol. 53, 1821-1830.

Hall, E.O. (1951) "The deformation and ageing of mild steel: iii. discussion of results," Proceedings of the Physical Society of London B, Vol. 64, 747-753.

Hansen, N. (1979) "The effect of grain size and strain on the tensile flow stress of copper at room temperature," In: Proceedings of the $5^{\text {th }}$ International Conference on the Strength of Metals and Alloys, Vol. 2, 849-854.

Hansen, N. (1983) "Flow stress and grain size dependence of non-ferrous metals and Alloys," Yield, Flow and Fracture of Polycrystals, Ed: T.N. Baker, Applied Science Publishers, London, pp. 311-350.

Hansen, N. (2004) "Hall-Petch relation and boundary strengthening" Scripta Materialia, Vol. 51, 801-806.

Haque, M.A. and Saif, M.T.A. (2003) "Strain gradient effect in nanoscale thin films," Acta Materialia, Vol. 51, 3053-3061.

Harder, J. (1999) "A crystallographic model for the study of local deformation processes in polycrystals," International Journal of Plasticity, Vol. 15, 605-624.

Hertzberg, R.W. (1983) Deformation and Fracture Mechanics of Engineering Materials $2^{\text {nd }}$ Edition, John Wiley \& Sons, New York.

Hill, R. and Rice, J.R. (1972) "Constitutive analysis of elastic-plastic crystals at arbitrary strain," Journal of the Mechanics and Physics of Solids, Vol. 20, 401-413.

Hirth, J.P. (1972) "The influence of grain boundaries on mechanical properties," Metallurgical Transactions, Vol. 3, 3047-3067.

Hook, R.E. and Hirth, J.P. (1967) "Deformation behavior of isoaxial bicrystals of Fe-3 percent Si,” Acta Metallurgica, Vol.15, 535-551. 
Hook, R.E. and Hirth, J.P. (1968) "Dislocation arrays in deformed silicon-iron bicrystals" Transactions of the Japan Institute of Metals, Vol. 9, 778.

Huang, H. and Spaepen, F. (2000) “Tensile testing of free-standing Cu, Ag, and Al then films and Ag/Cu multilayers" Acta Materialia, Vol. 48, 3261-3269.

Hutchinson, J.W. (1970) "Elastic-plastic behaviour of polycrystalline metals and composites," Proceedings of the Royal Society of London Series A-Mathematical and Physical Sciences, Vol. 319, 247-272.

Hutchinson, J.W. (1976) "Bounds and self-consistent estimates for creep of polycrystalline materials," Proceedings of the Royal Society of London Series AMathematical and Physical Sciences, Vol. 348, 101-127.

Johnston, T.L. and Feltner, C.E. (1970) "Grain size effects in the strain hardening of polycrysatls," Metallurgical Transactions, Vol. 1, 1161-1167.

Kalidindi, S.R. and Schoenfeld, S.E. (2000) "On the prediction of yield surfaces by the crystal plasticity models for polycrystals," Materials Science and Engineering, Vol. A239, 120-129.

Kocks, U.F. (1970) "The relation between polycrystal deformation and single-crystal deformation," Metallurgical Transactions, Vol. 1, 1121-1143.

Kocks, U.F. (1975) “Thermodynamics and kinetics of slip", Progress in Materials Science, Vol. 19, 1-281.

Kocks, U.F. and Mecking, H. (2003) "Physics and phenomenology of strain hardening: the FCC case," Progress in Materials Science, Vol. 48, 171-273.

Koiter, W.T. (1964) "Couple stresses in the theory of elasticity, I and II," Proceedings of the Koninklijke Nederlandse Akademie van Wetenschappen Series B - Physical Sciences, Vol. 67, 17-44.

Kroner, E. (1960) "Allgemeine kontinuumstheorie der versetzugnen und eigenspannungen,” Archive for Rational Mechanics and Analysis, Vol. 4, 273-334.

Kroner, E. (1966) "Continuum mechanics and range of atomic cohesion forces," International Journal of Fracture Mechanics, Vol. 2, 367.

Lee, E.H. (1969) "Elastic-plastic deformation at finite strains," ASME Journal of Applied Mechanics, Vol. 36, 1-6.

Lee, E.H. (1981) "Some comments on elastic-plastic analysis," International Journal of Solids and Structures, Vol. 17, 859-872. 
Li, J.C.M. (1963) "Petch relation and grain boundary sources," Transactions of the Metallurgical Society of AIME, Vol. 227, 239-247.

Ma, Q. and Clarke, D.R. (1995) "Size dependent hardness of silver single crystals", Journal of Materials Research, Vol. 10, 853-863.

MacKenzie, J.K. (1958) "Second paper on statistics associated with the random disorientation of cubes," Biometrika, Vol. 45, 229-240.

Mathur, K.K. and Dawson, P.D. (1989) "On modeling the development of crystallographic texture in bulk forming processes," International Journal of Plasticity, Vol. 5, 67-94.

McElhaney, K.W., Vlassak, J.J., Nix, W.D. (1998) "Determination of indenter tip geometry and indentation contact area for depth-sensing indentation experiments," Journal of Materials Research, Vol. 13, 1300-1306.

McGinty, R.D. (2001) "Multiscale representation of polycrystalline inelasticity," Ph.D. Dissertation, Georgia Institute of Technology.

McGinty, R.D. and McDowell, D.M. (2006) "A Semi-Implicit Integration Scheme for Rate Independent Finite Crystal Plasticity," International Journal of Plasticity, Vol. 22, 965-1170.

Meakin, J.D. and Petch, N.J. (1974) "Strain-hardening of polycrystals: the $\alpha$-brasses," Philosophical Magazine, Vol. 29, 1149-1156.

Mecking, H. and Kocks, U. (1981) "Kinetics of flow and strain-hardening," Acta Metellurgica, Vol. 29, 1865-1875.

Meric, L., Cailletaud, G., Gasperini, M. (1994) "F.E. calculations of copper bicrystal specimens submitted to tension-compression tests," Acta Metallurigca et Materialia, Vol. 42, 921-935.

Mindlin, R.D. and Tiersten, H.F. (1962) "Effects of couple-stresses in linear elasticity," Archive for Rational Mechanics and Analysis, Vol. 11, 414-448.

Mindlin, R.D. (1963) "Influence of couple stresses on stress concentrations," Experimental Mechanics, Vol. 3, 1-7.

Mindlin, R.D. (1965) "Second gradients of strain and surface tension in linear elasticity," International Journal of Solids and Structures, Vol. 1, 417-438.

Mughrabi, H. (2001) "On the role of strain gradients and long-range internal stresses in the composite model of crystal plasticity," Materials Science and Engineering A, Vol. $317,171-180$. 
Nemat-Nasser, S. (1979) "Decomposition of strain measures and their rates in finite deformation elastoplasticity," International Journal of Solids and Structures, Vol. 15, 155-166.

Nilsson, C. (1997) "Nonlocal strain softening bar revisited," International Journal of Solids and Structures, Vol. 34, 4399-4419.

Nilsson, C. (1999) “Author's closure," International Journal of Solids and Structures, Vol. 36, 3093-3100.

Nix, W.D. (1997) "Elastic and plastic properties of thin films on substrates: nanoindentation techniques," Materials Science and Engineering A, Vol. 234-236, 37-44.

Nix, W.D. and Gao, H. (1998) "Indentation size effects in crystalline materials: a law for strain gradient plasticity," Journal of the Mechanics and Physics of Solids, Vol. 46, 411425.

Nye, J.F. (1953) "Some geometrical relations in dislocated crystals," Acta Metallurgica, Vol. 1, 153-162.

Oliver, W.C. and Pharr, G.M. (1991) “An improved technique for determining hardness and elastic modulus using load and displacement sensing indentation experiments," Journal of Materials Research, Vol. 7, 1564-1583.

Peirce, D., Asaro, R.J., Needleman, A. (1982) "Analysis of nonuniform and localized deformation in ductile single crystals," Acta Metallurgica, Vol. 30, 1087-1119.

Petch, N.J. (1953) "The cleavage strength of polycrystals," Journal of the Iron and Steel Institute, Vol. 174, 25-28.

Pethica, J.B., Hutchings, R., Oliver, W.C. (1983) "Hardness measurement at penetration depths as small as 250 nm," Philosophical Magazine A, Vol. 48, 593-606.

Poole, W.J., Ashby, M.F., Fleck, N.A. (1996) "Micro-hardness of annealed and workhardened copper polycrystals”, Scripta Materialia, Vol. 34, 559-564.

Regueiro, R., Bammann, D.J., Marin, E.B., Garikipati, K. (2002) “A nonlocal model phenomenological anisotropic finite deformation plasticity model accounting for dislocation defects," Journal of Engineering Materials and Technology, Vol. 124, 380387.

Rogula, D. (1965) "Influence of spatial acoustic dispersion on dynamical properties of dislocations: I," Bulletin de l'Academie Polonaise des Sciences. Serie des Sciences Techniques, Vol. 13, 337-343. 
Schroder, J. and Miehe, C. (1997) "Aspects of computational rate-independent crystal plasticity," Computational Materials Science, Vol. 9, 168-176.

Sluys, L.J., and Estrin, Y. (2000) "The analysis of shear banding with a dislocation based gradient plasticity model," International Journal of Solids and Structures, Vol. 37, 71277142.

Soer, W.A., Aifantis, K.E., De Hosson, J.Th.M. (2005) "Incipient plasticity during nanoindentation at grain boundaries in body-centered cubic metals," Acta Materialia, Vol. 53, 4665-4676.

Soifer, Ya.M., Verdyan, A., Kazakevich, M., Rabkin, E. (2002) "Nanohardness of copper in the vicinity of grain boundaries," Scripta Materialia, Vol. 47, 799-804.

Steinmann, P.J. (1996) "Views on multiplicative elastoplasticity and the continuum theory of dislocations," International Journal of Engineering Science, Vol. 34, 17171735.

Stelmashenko, N.A., Walls, M.G., Brown, L.M., Milman, Y.V. (1993) "Microindentation of W and Mo oriented single crystals: an STM study," Acta Metallurigca et Materialia, Vol. 41, 2855-2865.

Stolken, J.S. and Evans, A.G. (1998) "A microbend test method for measuring the plasticity length scale,” Acta Materialia, Vol. 46, 5109-5115.

Stromberg, L. and Ristinmaa, M. (1996) "FE-formulation of a nonlocal plasticity theory," Computer Methods in Applied Mechanics and Engineering, Vol. 136, 127-144.

Suzuki, H., Ikeda, S., Takeuchi, S. (1956) "Deformation of thin copper crystals," Journal of the Physical Society of Japan, Vol. 11, 382-393.

Taylor, G.I. (1934) "Mechanism of plastic deformation of crystals. Part I.-Theoretical," Proceedings of the Royal Society of London Series A - Mathematical and Physical Sciences, Vol. 145, 362-387.

Thompson, A.W. and Baskes, M.I. (1973) "The influence of grain size on work hardening of face-centered cubic polycrystals," Philosophical Magazine, Vol. 28, 301308.

Thompson, A.W., Baskes, M.I., Flanagan, W.F. (1973) “The dependence of polycrystal work hardening on grain size," Acta Metellurgica, Vol. 21, 1017-1028.

Thompson, A.W. (1975) "Yielding in nickel as a function of grain or cell size," Acta Metallurgica, Vol. 23, 1337-1342. 
Toupin, R.A. (1962) "Elastic materials with couple stresses," Archive for Rational Mechanics and Analysis, Vol. 11, 384-414.

Toupin, R.A. (1964) "Theory of elasticity with couple-stresses," Archive for Rational Mechanics and Analysis, Vol. 17, 85-112.

Uchic, M.D., Dimiduk, D.M., Florando, J.N., Nix, W.D. (2004) "Sample Dimensions influence strength and crystal plasticity" Science, Vol. 305, 986-989.

Walgraef, D., and Aifantis, E.C. (1985) "On the formation and stability of dislocation patterns - 1: one-dimensional considerations", International Journal of Engineering Science, Vol. 23, 1351-1358.

Walgraef, D., and Aifantis, E.C. (1985) "On the formation and stability of dislocation patterns - III: three-dimensional considerations”, International Journal of Engineering Science, Vol. 23, 1365-1372.

Walgraef, D., and Aifantis, E.C. (1988) "Plastic instabilities, dislocation patterns, and nonequilibrium phenomena," Res Mechanica, Vol. 23, 161-195.

Weber, G. and Anand, L. (1990) "Finite deformation constitutive equations and a time integration procedure for isotropic, hyperelastic-visocplastic solids," Computer Methods in Applied Mechanics and Engineering, Vol. 79, 173-202.

Young, G.A., Lewis, N., Battige, C.K., Somers, R.A., Penik, M.A., Brewer, L.N., Othon, M. (2002) "Quantification of residual plastic strains in Ni-Cr-Nm-Nb GTAW welds via electron backscatter diffraction," In: $6^{\text {th }}$ International Trends in Welding Research Conference Proceedings, Pine Mountain GA, 912-917.

Yu, J., Maniatty, A. M., Knorr, D.B. (1997) "Model for predicting thermal stresses in thin polycrystalline films," Journal of the Mechanics and Physics of Solids, Vol. 45, 511-534.

Yun, G., Hwang, K.C., Huang, Y., Wu, P.D. (2005) “A reformulation of mechanismbased strain gradient plasticity,” Philosophical Magazine, Vol. 85, 4011-4029.

Zienkiewicz, O.C. and Taylor, R.L. (1994) The Finite Element Method, McGraw-Hill Book Company, London.

Zikry, M.A. and Kao, M. (1996) "Dislocation based multiple-slip crystalline constitutive formulation for finite strain plasticity," Scripta Materialia, Vol. 34, 1115-1121. 